\title{
Synthesis of Indenes by the Transition Metal-Mediated Carboannulation of Alkynes
}

\author{
Daohua Zhang, Zhijian Liu, Eul K. Yum and Richard C. Larock*
}

Department of Chemistry, Iowa State University, Ames, IA 50011

Larock@iastate.edu

Page(s)

General $2 S$

${ }^{1} \mathrm{H}$ and ${ }^{13} \mathrm{C}$ NMR Data 3S-21S

Copies of ${ }^{1} \mathrm{H}$ and ${ }^{13} \mathrm{C}$ NMR spectra 22S-156S 


\section{Supporting Information}

General. ${ }^{1} \mathrm{H}$ and ${ }^{13} \mathrm{C}$ NMR spectra were recorded at 300 and $75 \mathrm{M} \mathrm{Hz}$ or 400 and $100 \mathrm{M} \mathrm{Hz}$ respectively. Thin layer chromatography was performed using commercially prepared 40-mesh silica gel plates (Whatman K6F), and visualization was effected with short wavelength UV light $(254 \mathrm{~nm})$ and a basic $\mathrm{KMnO}_{4}$ solution [3 g of $\mathrm{KMnO}_{4}+20 \mathrm{~g}$ of $\mathrm{K}_{2} \mathrm{CO}_{3}+5 \mathrm{~mL}$ of $\mathrm{NaOH}(5 \%)+300 \mathrm{~mL}$ of $\mathrm{H}_{2} \mathrm{O}$ ]. All melting points are uncorrected. Low resolution mass spectra were recorded on a Finnigan TSQ700 triple quadrupole mass spectrometer. High resolution mass spectra were recorded on a Kratos MS50TC double focusing magnetic sector mass spectrometer using EI at $70 \mathrm{eV}$. The IR spectra were measured on a Bio-Rad FTS-7 spectrometer on salt plates. The product characterization data, and ${ }^{1} \mathrm{H}$ and ${ }^{13} \mathrm{C}$ NMR spectra for compounds (1-15) have been reported in our previous communication. ${ }^{1}$

General Procedure for the Synthesis of Indenes by the Palladium-Catalyzed Carboannulation of Internal Alkynes (Table 1).

For this experimental procedure, see the text.

General Procedure for the Synthesis of Indenes by the Copper(I)-Catalyzed Carboannulation of Alkynes (Table 2).

For this experimental procedure, see the text.

General Procedure for the Synthesis of Indenes by the Palladium-Catalyzed Carboannulation of Diethyl [2-(1-Alkynyl)phenyl]malonate by Organic Halides (Tables 3 and 4).

For this experimental procedure, see the text.

(1) Zhang, D.; Yum, E. K.; Liu, Z.; Larock, R. C. Org. Lett. 2005, 7, 4963. 


\section{Characterization Data:}

Diethyl [2-(oct-1-ynyl)phenyl]malonate (16). The indicated compound was obtained as a pale yellow oil in a 95\% yield: ${ }^{1} \mathrm{H} \mathrm{NMR}\left(\mathrm{CDCl}_{3}\right) \delta 0.87(\mathrm{t}, J=7.2 \mathrm{~Hz}$, 3H), 1.25-1.32 (m, 10H), 1.44 (m, 2H), 1.59 (m, 2H), 2.41 (t, $J=7.2 \mathrm{~Hz}, 2 \mathrm{H}), 4.21$ (m, 4H), 5.30 (s, 1H), 7.24 (m, 2H), 7.41 (m, 2H); ${ }^{13} \mathrm{C}$ NMR $\left(\mathrm{CDCl}_{3}\right) \delta 14.1,19.6$, 22.6, 28.6, 28.7, 31.4, 55.7, 61.8, 78.2, 95.9, 124.6, 127.8, 127.9, 128.4, 132.0, 134.6, 168.3; IR $\left(\mathrm{CHCl}_{3}, \mathrm{~cm}^{-1}\right)$ 3058, 2982, 1751, 1734, 1266; HRMS calcd for $\mathrm{C}_{21} \mathrm{H}_{28} \mathrm{O}_{4}$ 344.1988, found 344.1992.

Diethyl 2-n-hexyl-1H-indene-1,1-dicarboxylate (17). The indicated compound was obtained as a pale yellow oil in a 96\% yield: ${ }^{1} \mathrm{H}$ NMR $\left(\mathrm{CDCl}_{3}\right) \delta 0.91(\mathrm{~m}, 3 \mathrm{H}), 1.25$ (t, $J=7.2 \mathrm{~Hz}, 6 \mathrm{H}), 1.35$ (m, 6H), 1.66 (m, 2H), 2.51 (m, 2H), 4.21 (m, 4H), 6.63 (t, $J$ $=1.8 \mathrm{~Hz}, 1 \mathrm{H}), 7.26(\mathrm{~m}, 3 \mathrm{H}), 7.57(\mathrm{~m}, 1 \mathrm{H}) ;{ }^{13} \mathrm{C} \mathrm{NMR}\left(\mathrm{CDCl}_{3}\right) \delta 14.2,14.3,22.9,28.1$, 28.7, 29.5, 30.0, 62.1, 72.2, 120.8, 125.0, 125.4, 128.8, 130.0, 140.9, 144.7, 148.5, 168.5; IR $\left(\mathrm{CHCl}_{3}, \mathrm{~cm}^{-1}\right)$ 3048, 2983, 1758, 1471; HRMS calcd for $\mathrm{C}_{21} \mathrm{H}_{28} \mathrm{O}_{4}$ 334.1988, found 334.1994.

Diethyl [2-(hex-1-ynyl)phenyl]malonate (18). The indicated compound was obtained as a pale yellow oil in a 93\% yield: ${ }^{1} \mathrm{H}$ NMR $\left(\mathrm{CDCl}_{3}\right) \delta 0.95(\mathrm{~m}, 3 \mathrm{H}), 1.25$ (m, 6H), 1.49 (m, 2H), 1.59 (m, 2H), 2.44 (t, $J=6.9 \mathrm{~Hz}, 2 \mathrm{H}), 4.22$ (m, 4H), 5.32 (s, 1H), 7.26 (m, 2H), 7.42 (m, 2H); ${ }^{13} \mathrm{C} \mathrm{NMR}\left(\mathrm{CDCl}_{3}\right) \delta 13.7,14.1,19.2,22.0,30.7$, 55.7, 61.8, 78.2, 95.8, 124.6, 127.8, 127.9, 128.4, 132.0, 134.7, 168.3; IR $\left(\mathrm{CHCl}_{3}\right.$, $\mathrm{cm}^{-1}$ ) 3057, 2982, 1753, 1733, 1266; HRMS calcd for $\mathrm{C}_{19} \mathrm{H}_{24} \mathrm{O}_{4} 316.1675$, found 316.1678.

Diethyl 2-n-butyl-1H-indene-1,1-dicarboxylate (19). The indicated compound was obtained as a pale yellow oil in a 92\% yield: ${ }^{1} \mathrm{H}$ NMR $\left(\mathrm{CDCl}_{3}\right) \delta 1.00(\mathrm{t}, J=7.2 \mathrm{~Hz}$, 3H), 1.27 (t, $J=7.2 \mathrm{~Hz}, 6 \mathrm{H}), 1.48$ (m, 2H), 1.70 (m, 2H), 2.56 (m, 2H), 4.23 (m, 4H), $6.66(\mathrm{t}, J=1.6 \mathrm{~Hz}, 1 \mathrm{H}), 7.18-7.33(\mathrm{~m}, 3 \mathrm{H}), 7.61(\mathrm{~d}, J=7.6 \mathrm{~Hz}, 1 \mathrm{H}) ;{ }^{13} \mathrm{C} \mathrm{NMR}$ 
$\left(\mathrm{CDCl}_{3}\right) \delta 14.0,14.1,22.7,28.3,30.1,61.9,72.0,120.6,124.8,125.3,128.6,129.7$ 140.7, 144.5, 148.3, 168.3; IR $\left(\mathrm{CHCl}_{3}, \mathrm{~cm}^{-1}\right)$ 2959, 2932, 1732, 1467, 1235; HRMS calcd for $\mathrm{C}_{19} \mathrm{H}_{24} \mathrm{O}_{4} 316.1675$, found 316.1678.

Diethyl [2-(3-methoxyprop-1-ynyl)phenyl]malonate (20). The indicated compound was obtained as a pale yellow oil in an $81 \%$ yield: ${ }^{1} \mathrm{H}$ NMR $\left(\mathrm{CDCl}_{3}\right) \delta$ 1.27 (t, $J=7.2 \mathrm{~Hz}, 6 \mathrm{H}), 3.47$ (s, 3H), 4.23 (m, 4H), 4.36 (s, 2H), 5.29 (s, 1H), 7.29 (m, 2H), 7.49 (dd, $J=1.2,7.8 \mathrm{~Hz}, 2 \mathrm{H}) ;{ }^{13} \mathrm{C} \mathrm{NMR}\left(\mathrm{CDCl}_{3}\right) \delta 14.1,55.7,57.7,60.4$, 61.9, 84.0, 90.1, 123.2, 128.0, 128.7, 128.9, 132.5, 135.0, 168.1; IR $\left(\mathrm{CHCl}_{3}, \mathrm{~cm}^{-1}\right)$ 3054, 2928, 1749, 1713, 1266; HRMS calcd for $\mathrm{C}_{17} \mathrm{H}_{20} \mathrm{O}_{5}$ 304.1311, found 304.1316.

Diethyl 2-methoxymethyl-1H-indene-1,1-dicarboxylate (21). The indicated compound was obtained as a pale yellow oil in a 91\% yield: ${ }^{1} \mathrm{H}$ NMR $\left(\mathrm{CDCl}_{3}\right) \delta$ 1.23 (t, $J=7.2 \mathrm{~Hz}, 3 \mathrm{H}), 3.41$ (s, 3H), 4.20 (m, 4H), 4.43 (d, $J=1.6 \mathrm{~Hz}, 2 \mathrm{H}), 6.88$ (d, $J=1.2 \mathrm{~Hz}, 1 \mathrm{H}), 7.25$ (m, 3H), 7.61 (d, $J=7.6 \mathrm{~Hz}, 1 \mathrm{H}) ;{ }^{13} \mathrm{C} \mathrm{NMR}\left(\mathrm{CDCl}_{3}\right) \delta 14.0$, 58.6, 62.2, 69.6, 70.5, 121.3, 125.1, 126.0, 128.7, 132.1, 140.8, 143.3, 143.5, 167.8; IR $\left(\mathrm{CHCl}_{3}, \mathrm{~cm}^{-1}\right)$ 2984, 1733, 1464, 1244; HRMS calcd for $\mathrm{C}_{17} \mathrm{H}_{20} \mathrm{O}_{5}$ 304.1311, found 304.1316 .

Diethyl [2-(11-hydroxyundec-1-ynyl)phenyl]malonate (22). The indicated compound was obtained as a pale yellow oil in a 98\% yield: ${ }^{1} \mathrm{H}$ NMR $\left(\mathrm{CDCl}_{3}\right) \delta$ 1.27 (t, $J=7.2 \mathrm{~Hz}, 6 \mathrm{H}), 1.33-1.64$ (m, 15H), 2.43 (t, $J=7.2 \mathrm{~Hz}, 2 \mathrm{H}), 3.62$ (t, $J=6.6$ $\mathrm{Hz}, 2 \mathrm{H}), 4.23$ (m, 4H), 5.31 (s, 1H), 7.27 (m, 2H), 7.43 (m, 2H); ${ }^{13} \mathrm{C} \mathrm{NMR}\left(\mathrm{CDCl}_{3}\right) \delta$ 14.3, 19.7, 26.0, 28.8, 29.1, 29.3, 29.6, 29.7, 33.0, 55.9, 62.0, 63.1, 76.9, 96.1, 124.8, 128.0, 128.1, 128.6, 132.2, 134.8, 168.5; IR $\left(\mathrm{CHCl}_{3}, \mathrm{~cm}^{-1}\right)$ 3421, 3054, 2931, 1748, 1732, 1265; HRMS calcd for $\mathrm{C}_{24} \mathrm{H}_{34} \mathrm{O}_{5} 402.2406$, found 402.2413 .

Diethyl 2-(9-hydroxy-1-nonyl)-1H-indene-1,1-dicarboxylate (23). The indicated compound was obtained as a pale yellow oil in a $79 \%$ yield: ${ }^{1} \mathrm{H}$ NMR $\left(\mathrm{CDCl}_{3}\right) \delta$ 1.22 (t, $J=7.2 \mathrm{~Hz}, 6 \mathrm{H}), 1.30-1.66$ (m, 15H), 2.47 (t, $J=7.2 \mathrm{~Hz}, 2 \mathrm{H}), 3.59$ (t, $J=6.8$ 
Hz, 2H), 4.17 (m, 4H), 6.60 (s, 1H), 7.15 (dt, $J=1.2,7.2 \mathrm{~Hz}, 1 \mathrm{H}$ ), 7.20 (d, $J=7.2 \mathrm{~Hz}$, 1H), 7.26 (t, $J=7.2 \mathrm{~Hz}, 1 \mathrm{H}), 7.55$ (d, $J=7.6 \mathrm{~Hz}, 1 \mathrm{H}) ;{ }^{13} \mathrm{C} \mathrm{NMR}\left(\mathrm{CDCl}_{3}\right) \delta 14.0$, 25.8, 27.9, 28.5, 29.4, 29.5, 29.6, 32.8, 61.9, 63.0, 72.0, 120.6, 124.8, 125.3, 128.6, 129.7, 140.7, 144.5, 148.3, 168.5; IR $\left(\mathrm{CHCl}_{3}, \mathrm{~cm}^{-1}\right)$ 2930, 1733, 1725, 1242; HRMS calcd for $\mathrm{C}_{24} \mathrm{H}_{34} \mathrm{O}_{5} 402.2406$, found 402.2413.

Diethyl [2-(11-methoxy-11-oxoundec-1-ynyl)phenyl]malonate (24). The indicated compound was obtained as a pale yellow oil in an $86 \%$ yield: ${ }^{1} \mathrm{H}$ NMR $\left(\mathrm{CDCl}_{3}\right) \delta$ 1.26 (dt, $J=1.2,7.2 \mathrm{~Hz}, 6 \mathrm{H}), 1.33-1.64(\mathrm{~m}, 12 \mathrm{H}), 2.30$ (t, $J=7.2 \mathrm{~Hz}, 2 \mathrm{H}), 2.43(\mathrm{t}, J=$ $7.2 \mathrm{~Hz}, 2 \mathrm{H}), 3.66$ (s, 3H), 4.23 (m, 4H), 5.31 (s, 1H), 7.27 (m, 2H), 7.42 (t, $J=7.8$ $\mathrm{Hz}, 2 \mathrm{H}) ;{ }^{13} \mathrm{C} \mathrm{NMR}\left(\mathrm{CDCl}_{3}\right) \delta 14.1,19.5,24.9,28.6,28.9,29.0,29.1,29.2,34.0,51.4$, 55.7, 61.7, 78.2, 95.8, 124.5, 127.8, 127.9, 128.4, 132.0, 134.6, 168.2, 174.2; IR $\left(\mathrm{CHCl}_{3}, \mathrm{~cm}^{-1}\right)$ 3056, 2934, 1734, 1266; HRMS calcd for $\mathrm{C}_{25} \mathrm{H}_{34} \mathrm{O}_{6} 430.2355$, found 430.2363.

Diethyl 2-(9-methoxy-9-oxo-1-nonyl)-1H-indene-1,1-dicarboxylate (25). The indicated compound was obtained as a pale yellow oil in an 83\% yield: ${ }^{1} \mathrm{H}$ NMR $\left(\mathrm{CDCl}_{3}\right) \delta 1.22(\mathrm{dt}, J=1.2,7.2 \mathrm{~Hz}, 6 \mathrm{H}), 1.36-1.64(\mathrm{~m}, 12 \mathrm{H}), 2.28(\mathrm{t}, J=7.2 \mathrm{~Hz}, 2 \mathrm{H})$, 2.48 (m, 2H), 3.64 (s, 3H), 4.18 (m, 4H), 6.60 (s, 1H), 7.14-7.26 (m, 3H), 7.54 (d, $J=$ 7.2 Hz, 1H); ${ }^{13} \mathrm{C} \mathrm{NMR}\left(\mathrm{CDCl}_{3}\right) \delta 14.0,25.0,27.9,28.5,29.2,29.3,29.4,29.5,34.1$, 54.5, 61.9, 72.0, 120.6, 124.8, 125.3, 128.6, 129.7, 140.7, 144.5, 148.2, 168.3, 174.4; IR $\left(\mathrm{CHCl}_{3}, \mathrm{~cm}^{-1}\right)$ 2982, 1734, 1718, 1240; HRMS calcd for $\mathrm{C}_{25} \mathrm{H}_{34} \mathrm{O}_{6}$ 430.2355, found 430.2362 .

Diethyl [2-(3,3-diethoxyprop-1-ynyl)phenyl]malonate (26). The indicated compound was obtained as a pale yellow oil in a $75 \%$ yield: ${ }^{1} \mathrm{H}$ NMR $\left(\mathrm{CDCl}_{3}\right) \delta$ 1.22 (m, 12H), 3.66 (m, 2H), 3.78 (m, 2H), 4.17 (m, 4H), 5.22 (s, 1H), 5.47 (s, 1H), $7.23(\mathrm{dt}, J=1.2,7.8 \mathrm{~Hz}, 1 \mathrm{H}), 7.22$ (dt, $J=1.2,7.8 \mathrm{~Hz}, 1 \mathrm{H}), 7.46(\mathrm{~m}, 2 \mathrm{H}) ;{ }^{13} \mathrm{C} \mathrm{NMR}$ $\left(\mathrm{CDCl}_{3}\right) \delta 14.0,15.2,55.5,61.0,61.9,82.7,89.5,91.8,122.4,127.9,128.8,129.2$, 
132.6, 135.0, 167.0; IR $\left(\mathrm{CHCl}_{3}, \mathrm{~cm}^{-1}\right)$ 3054, 2981, 1749, 1733, 1266; HRMS calcd for $\mathrm{C}_{20} \mathrm{H}_{26} \mathrm{O}_{6}$ 362.1736, found 362.17427.

Diethyl 2-diethoxymethyl-1H-indene-1,1-dicarboxylate (27). The indicated compound was obtained as a pale yellow oil in a $58 \%$ yield: ${ }^{1} \mathrm{H}$ NMR $\left(\mathrm{CDCl}_{3}\right) \delta$ 1.23 (m, 12H), 3.61 (m, 2H), 3.73 (m, 2H), 4.18 (m, 4H), 5.91 (d, J = $1.2 \mathrm{~Hz}, 1 \mathrm{H})$, 7.00 (s, 1H), 7.24 (m, 1H), $7.31(\mathrm{~m}, 1 \mathrm{H}), 7.63$ (d, $J=7.8 \mathrm{~Hz}, 2 \mathrm{H}) ;{ }^{13} \mathrm{C} \mathrm{NMR}\left(\mathrm{CDCl}_{3}\right)$ $\delta$ 13.9, 15.3, 61.9, 62.1, 70.1, 99.1, 121.8, 124.9, 126.4, 128.6, 132.7, 141.7, 143.1, 144.3, 167.9; IR $\left(\mathrm{CHCl}_{3}, \mathrm{~cm}^{-1}\right)$ 3055, 2980, 1732, 1682, 1266; HRMS calcd for $\mathrm{C}_{20} \mathrm{H}_{26} \mathrm{O}_{6}$ 362.1729, found 362.1734.

Diethyl [2-(5-cyanopent-1-ynyl)phenyl]malonate (28). The indicated compound was obtained as a pale yellow oil in a 62\% yield: ${ }^{1} \mathrm{H}$ NMR $\left(\mathrm{CDCl}_{3}\right) \delta 1.27(\mathrm{t}, J=7.2$ Hz, 6H), 1.98 (m, 2H), 2.57 (t, $J=7.2 \mathrm{~Hz}, 2 \mathrm{H}), 2.63$ (t, $J=7.2 \mathrm{~Hz}, 2 \mathrm{H}), 4.24$ (m, 4H), 5.23 (s, 1H), 7.27 (dt, $J=1.2,7.2 \mathrm{~Hz}, 1 \mathrm{H}), 7.33$ (dt, $J=1.6,7.8 \mathrm{~Hz}, 1 \mathrm{H}), 7.45$ (dt, $J=$ 1.2, $8.0 \mathrm{~Hz}, 2 \mathrm{H}) ;{ }^{13} \mathrm{C} \mathrm{NMR}\left(\mathrm{CDCl}_{3}\right) \delta 14.1,16.2$, 18.7, 24.6, 55.9, 61.9, 80.8, 92.4, 119.2, 123.7, 128.0, 128.4, 128.7, 132.2, 134.7, 168.1; IR $\left(\mathrm{CHCl}_{3}, \mathrm{~cm}^{-1}\right)$ 2984, 2254, 1748, 1732; $\mathrm{C}_{19} \mathrm{H}_{21} \mathrm{NO}_{4}$ 327.1471, found 327.1475.

Diethyl 2-(3-cyano-1-propyl)-1H-indene-1,1-dicarboxylate (29). The indicated compound was obtained as a pale yellow oil in a $68 \%$ yield: ${ }^{1} \mathrm{H}$ NMR $\left(\mathrm{CDCl}_{3}\right) \delta$ 1.24 (t, $J=7.2 \mathrm{~Hz}, 6 \mathrm{H}), 2.04$ (t, $J=7.2 \mathrm{~Hz}, 2 \mathrm{H}), 2.45$ (t, $J=7.2,2 \mathrm{H}), 2.67$ (m, 2H), 4.20 (m, 4H), 6.64 (s, 1H), 7.24 (m, 3H), 7.57 (d, $J=7.8 \mathrm{~Hz}, 1 \mathrm{H}) ;{ }^{13} \mathrm{C}$ NMR $\left(\mathrm{CDCl}_{3}\right)$ $\delta$ 14.0, 16.8, 23.9, 27.6, 62.1, 71.9, 119.6, 120.9, 125.1, 125.8, 128.8, 130.9, 140.5, 143.7, 145.1, 167.9; IR $\left(\mathrm{CHCl}_{3}, \mathrm{~cm}^{-1}\right)$ 3253, 2985, 2253, 1721, 1265; HRMS calcd for $\mathrm{C}_{19} \mathrm{H}_{21} \mathrm{NO}_{4}$ 327.1471, found 327.1475.

Diethyl [2-(3,3-dimethylbut-1-ynyl)phenyl]malonate (30). The indicated compound was obtained as a pale yellow oil in a 94\% yield: ${ }^{1} \mathrm{H}$ NMR $\left(\mathrm{CDCl}_{3}\right) \delta$ 1.27 (dt, $J=0.8,7.2 \mathrm{~Hz}, 6 \mathrm{H}), 1.33$ (d, $J=1.2 \mathrm{~Hz}, 9 \mathrm{H}), 4.24$ (m, 4H), 5.28 (s, 1H), 
$7.26(\mathrm{~m}, 2 \mathrm{H}), 7.40(\mathrm{t}, J=7.6 \mathrm{~Hz}, 2 \mathrm{H}) ;{ }^{13} \mathrm{C} \mathrm{NMR}\left(\mathrm{CDCl}_{3}\right) \delta 14.1,28.2,30.9,55.9$, 61.8, 104.0, 124.5, 127.8, 127.9, 128.3, 131.8, 134.8, 168.4; IR $\left(\mathrm{CHCl}_{3}, \mathrm{~cm}^{-1}\right)$ 3053, 2970, 1749, 1736, 1265; HRMS calcd for $\mathrm{C}_{19} \mathrm{H}_{24} \mathrm{O}_{4}$ 316.1675, found 316.1678.

Diethyl 2-tert-butyl-1H-indene-1,1-dicarboxylate (31). The indicated compound was obtained as a pale yellow oil in a 69\% yield: ${ }^{1} \mathrm{H}$ NMR $\left(\mathrm{CDCl}_{3}\right) \delta 1.14(\mathrm{t}, J=7.2$ Hz, 6H), 1.28 (s, 9H), 4.11 (m, 4H), 6.78 (s, 1H), 7.11 (dt, $J=1.2,7.6 \mathrm{~Hz}, 1 \mathrm{H}), 7.23$ (m, 2H), 7.41 (d, $J=7.6 \mathrm{~Hz}, 1 \mathrm{H}) ;{ }^{13} \mathrm{C} \mathrm{NMR}\left(\mathrm{CDCl}_{3}\right) \delta 13.8,31.2,34.8,61.6,70.6$, 120.6, 123.1, 125.4, 128.4, 131.2, 142.3, 144.1, 157.5, 168.8; IR $\left(\mathrm{CHCl}_{3}, \mathrm{~cm}^{-1}\right) 3057$, 2965, 1750, 1715, 1222; HRMS calcd for $\mathrm{C}_{19} \mathrm{H}_{24} \mathrm{O}_{4}$ 316.1675, found 316.1678.

Diethyl [2-(trimethylsilylethynyl)phenyl]malonate (32). The indicated compound was obtained as a pale yellow oil in a 98\% yield: ${ }^{1} \mathrm{H}$ NMR $\left(\mathrm{CDCl}_{3}\right) \delta 0.27$ (s, 9H), 1.28 (t, $J=7.2 \mathrm{~Hz}, 6 \mathrm{H}), 4.25$ (m, 4H), 5.32 (s, 1H), 7.29 (dt, $J=1.2,7.2 \mathrm{~Hz}, 1 \mathrm{H})$, 7.35 (dt, $J=1.2,7.5 \mathrm{~Hz}, 1 \mathrm{H}), 7.44$ (dd, $J=1.2,7.8 \mathrm{~Hz}, 1 \mathrm{H}$ ), 7.49 (dd, $J=1.2,7.2 \mathrm{~Hz}$, $1 \mathrm{H}) ;{ }^{13} \mathrm{C} \mathrm{NMR}\left(\mathrm{CDCl}_{3}\right) \delta 0.08,14.3,56.0,62.0,100.2,102.6,123.8,128.0,128.6$, 129.0, 132.4, 135.5, 168.3; IR $\left(\mathrm{CHCl}_{3}, \mathrm{~cm}^{-1}\right)$ 3054, 2984, 2156, 1749, 1732, 1266; HRMS calcd for $\mathrm{C}_{18} \mathrm{H}_{24} \mathrm{SiO}_{4} 332.1444$, found 332.1449.

Diethyl [2-(cyclohexylethynyl)phenyl]malonate (34). The indicated compound was obtained as a pale yellow oil in a 91\% yield: ${ }^{1} \mathrm{H}$ NMR $\left(\mathrm{CDCl}_{3}\right) \delta 1.27(\mathrm{t}, J=7.2$ Hz, 6H), 1.37 (m, 3H), 1.57 (m, 3H), 1.76 (m, 2H), 1.86 (m, 2H), 2.64 (m, 1H), 4.23 (m, 4H), 5.33 (s, 1H), 7.26 (m, 2H), 7.41 (d, $J=7.8 \mathrm{~Hz}, 2 \mathrm{H}) ;{ }^{13} \mathrm{C} \mathrm{NMR}\left(\mathrm{CDCl}_{3}\right) \delta$ 14.8, 24.8, 25.9, 29.8, 32.6, 55.8, 61.8, 78.2, 99.9, 124.6, 127.8, 127.9, 128.3, 131.9, 134.7, 168.4; IR $\left(\mathrm{CHCl}_{3}, \mathrm{~cm}^{-1}\right)$ 3054, 2933, 1749, 1733, 1265; HRMS calcd for $\mathrm{C}_{21} \mathrm{H}_{26} \mathrm{O}_{4}$ 342.1831, found 342.1837.

Diethyl 2-cyclohexyl-1H-indene-1,1-dicarboxylate (35). The indicated compound was obtained as a pale yellow oil in a 92\% yield: ${ }^{1} \mathrm{H}$ NMR $\left(\mathrm{CDCl}_{3}\right) \delta 1.23-1.35(\mathrm{~m}$, 11H), 1.70-1.91 (m, 5H), 2.53 (m, 1H), 4.18 (m, 4H), 6.64 (s, 1H), 7.15 (dt, $J=1.2$, 
$7.8 \mathrm{~Hz}, 1 \mathrm{H}$ ), 7.20 (d, $J=6.8 \mathrm{~Hz}, 1 \mathrm{H}), 7.26$ (dt, $J=1.2,7.6 \mathrm{~Hz}, 1 \mathrm{H}$ ), 7.54 (dd, $J=1.2$, $7.6 \mathrm{~Hz}, 1 \mathrm{H}) ;{ }^{13} \mathrm{C} \mathrm{NMR}\left(\mathrm{CDCl}_{3}\right) \delta 14.0,26.3,26.9,34.6,38.1,61.9,71.9,120.7$, 124.8, 125.4, 128.6, 129.4, 140.2, 144.6, 153.9, 168.5; IR $\left(\mathrm{CHCl}_{3}, \mathrm{~cm}^{-1}\right) 3019,2929$, 1733, 1216; HRMS calcd for $\mathrm{C}_{21} \mathrm{H}_{26} \mathrm{O}_{4}$ 342.1831, found 342.1837.

Diethyl [2-(1-cyclohexenylethynyl)phenyl]malonate (36). The indicated compound was obtained as a pale yellow oil in a 90\% yield: ${ }^{1} \mathrm{H}$ NMR $\left(\mathrm{CDCl}_{3}\right) \delta$ 1.24 (t, $J=7.2 \mathrm{~Hz}, 6 \mathrm{H}), 1.64$ (m, 4H), 2.13 (m, 2H), 2.22 (m, 2H), 4.22 (m, 4H), 5.26 (m, 1H), $6.22(\mathrm{~m}, 1 \mathrm{H}), 7.28(\mathrm{~m}, 2 \mathrm{H}), 7.42(\mathrm{~m}, 2 \mathrm{H}) ;{ }^{13} \mathrm{C} \mathrm{NMR}\left(\mathrm{CDCl}_{3}\right) \delta 14.2,21.7$, 22.5, 26.0, 29.3, 56.1, 62.0, 84.4, 96.8, 120.8, 124.5, 128.0, 128.3, 128.6, 132.0, 134.8, 135.9, 168.4; IR $\left(\mathrm{CHCl}_{3}, \mathrm{~cm}^{-1}\right)$ 3056, 2936, 1751, 1733, 1266; HRMS calcd for $\mathrm{C}_{21} \mathrm{H}_{24} \mathrm{O}_{4} 340.1675$, found 340.1678 .

Diethyl 2-(1-cyclohexenyl)-1H-indene-1,1-dicarboxylate (37). The indicated compound was obtained as a pale yellow oil in an $85 \%$ yield: ${ }^{1} \mathrm{H}$ NMR $\left(\mathrm{CDCl}_{3}\right) \delta$ 1.15 (t, $J=7.2 \mathrm{~Hz}, 6 \mathrm{H}), 1.61$ (m, 2H), 1.74 (m, 2H), 2.20 (m, 2H), 2.36 (m, 2H), 4.17 (m, 4H), 6.04 (t, $J=4.2 \mathrm{~Hz}, 1 \mathrm{H}), 6.79$ (s, 1H), 7.16 (m, 1H), 7.27 (m, 2H), 7.53 (dd, $J$ $=0.9,7.5 \mathrm{~Hz}, 1 \mathrm{H}) ;{ }^{13} \mathrm{C} \mathrm{NMR}\left(\mathrm{CDCl}_{3}\right) \delta 13.9,22.1,22.8,26.1,26.7,61.7,69.7,122.3$, 123.6, 125.7, 128.3, 128.7, 129.0, 130.5, 142.2, 144.2, 147.9, 168.7; IR $\left(\mathrm{CHCl}_{3}, \mathrm{~cm}^{-1}\right)$ 2981, 1734, 1237; HRMS calcd for $\mathrm{C}_{21} \mathrm{H}_{24} \mathrm{O}_{4} 340.1675$, found 340.1678 .

Diethyl [2-(phenylethynyl)phenyl]malonate (38). The indicated compound was obtained as a pale yellow oil in a 98\% yield: ${ }^{1} \mathrm{H}$ NMR $\left(\mathrm{CDCl}_{3}\right) \delta 1.27(\mathrm{t}, J=7.2 \mathrm{~Hz}$, 6H), 4.25 (m, 4H), 5.40 (s, 1H), 7.35 (m, 5H), 7.49 (m, 1H), 7.57 (m, 3H); ${ }^{13} \mathrm{C} \mathrm{NMR}$ $\left(\mathrm{CDCl}_{3}\right) \delta 14.1,56.1,61.9,86.9,94.6,122.9,123.8,128.0,128.5,128.7,128.8,131.6$, 132.1, 135.0, 168.2; IR $\left(\mathrm{CHCl}_{3}, \mathrm{~cm}^{-1}\right)$ 3055, 2986, 1749, 1733, 1266; HRMS calcd for $\mathrm{C}_{21} \mathrm{H}_{20} \mathrm{O}_{4}$ 336.1362, found 336.1367.

Diethyl 2-phenyl-1H-indene-1,1-dicarboxylate (39). The indicated compound was obtained as a pale yellow oil in a $61 \%$ yield: ${ }^{1} \mathrm{H}$ NMR $\left(\mathrm{CDCl}_{3}\right) \delta 1.07(\mathrm{t}, J=7.2 \mathrm{~Hz}$, 
6H), 4.14 (m, 4H), 7.24-7.36 (m, 7H), 7.62 (m, 3H); ${ }^{13} \mathrm{C} \mathrm{NMR}\left(\mathrm{CDCl}_{3}\right) \delta 13.8,62.1$, 70.9, 121.7, 124.2, 126.3, 127.4, 127.9, 128.2, 128.9, 131.5, 134.1, 142.1, 143.7, 145.7, 168.3; IR $\left(\mathrm{CHCl}_{3}, \mathrm{~cm}^{-1}\right)$ 3019, 2988, 1734, 1217; HRMS calcd for $\mathrm{C}_{21} \mathrm{H}_{20} \mathrm{O}_{4}$ 336.1362, found 336.1366.

Diethyl (2-\{[4-(ethoxycarbonyl)phenyl]ethynyl\}phenyl)malonate (40). The indicated compound was obtained as a pale yellow oil in a 63\% yield: ${ }^{1} \mathrm{H}$ NMR $\left(\mathrm{CDCl}_{3}\right) \delta 1.23(\mathrm{t}, J=7.2 \mathrm{~Hz}, 6 \mathrm{H}), 1.37$ (t, $\left.J=7.2 \mathrm{~Hz}, 3 \mathrm{H}\right), 4.22(\mathrm{~m}, 4 \mathrm{H}), 4.35$ (q, $J=$ $7.2 \mathrm{~Hz}, 2 \mathrm{H}), 5.33$ (s, 1H), 7.32 (dt, $J=1.2,7.6 \mathrm{~Hz}, 1 \mathrm{H}), 7.35$ (dt, $J=1.2,7.6 \mathrm{~Hz}, 1 \mathrm{H})$, 7.46 (d, $J=7.6 \mathrm{~Hz}, 1 \mathrm{H}), 7.56(\mathrm{~m}, 3 \mathrm{H}), 8.00(\mathrm{~d}, J=7.6 \mathrm{~Hz}, 2 \mathrm{H}) ;{ }^{13} \mathrm{C} \mathrm{NMR}\left(\mathrm{CDCl}_{3}\right) \delta$ 14.1, 14.4, 56.1, 61.2, 62.0, 89.7, 93.8, 123.2, 127.4, 128.1, 128.8, 129.2, 129.6, 130.2, 131.5, 132.3, 135.2, 166.0, 168.0; IR $\left(\mathrm{CHCl}_{3}, \mathrm{~cm}^{-1}\right)$ 3032, 2988, 1726, 1266; HRMS calcd for $\mathrm{C}_{24} \mathrm{H}_{24} \mathrm{O}_{6} 408.1573$, found 408.1580.

Diethyl 2-[4-(ethoxycarbonyl)phenyl]-1H-indene-1,1-dicarboxylate (41). The indicated compound was obtained as a pale yellow oil in a $65 \%$ yield: ${ }^{1} \mathrm{H}$ NMR $\left(\mathrm{CDCl}_{3}\right) \delta 1.06(\mathrm{t}, J=7.2 \mathrm{~Hz}, 6 \mathrm{H}), 1.38(\mathrm{t}, J=7.2 \mathrm{~Hz}, 3 \mathrm{H}), 4.12(\mathrm{~m}, 4 \mathrm{H}), 4.36(\mathrm{q}, J=$ $7.2 \mathrm{~Hz}, 2 \mathrm{H}), 7.24-7.40$ (m, 4H), 7.65 (d, $J=8.4 \mathrm{~Hz}, 3 \mathrm{H}), 8.00$ (m, 2H); ${ }^{13} \mathrm{C} \mathrm{NMR}$ $\left(\mathrm{CDCl}_{3}\right) \delta 13.8,14.4,61.0,62.2,70.8,122.1,124.4,127.0,127.1,129.1,129.4,129.5$, 133.6, 138.4, 142.3, 143.1, 144.5, 166.4, 168.0; IR $\left(\mathrm{CHCl}_{3}, \mathrm{~cm}^{-1}\right)$ 3020, 2982, 1719, 1724, 1283; HRMS calcd for $\mathrm{C}_{24} \mathrm{H}_{24} \mathrm{O}_{6} 408.1573$, found 408.1580 .

Diethyl \{2-[(4-methoxyphenyl)ethynyl]phenyl\}malonate (42). The indicated compound was obtained as a pale yellow oil in an $86 \%$ yield: ${ }^{1} \mathrm{H}$ NMR $\left(\mathrm{CDCl}_{3}\right) \delta$ 1.25 (t, $J=7.2 \mathrm{~Hz}, 6 \mathrm{H}$ ), 3.80 (s, 3H), 4.22 (m, 4H), 5.38 (s, 1H), 6.87 (dt, $J=2.0,9.2$ $\mathrm{Hz}, 2 \mathrm{H}), 7.31$ (m, 2H), 7.46 (m, 3H), 7.53 (m, 1H); ${ }^{13} \mathrm{C} \mathrm{NMR}\left(\mathrm{CDCl}_{3}\right) \delta 14.1,55.4$, 56.2, 61.9, 85.7, 94.8, 114.1, 115.0, 124.1, 128.0, 128.4, 128.6, 131.9, 133.1, 134.8, 160.0, 168.3; IR $\left(\mathrm{CHCl}_{3}, \mathrm{~cm}^{-1}\right)$ 3056, 2985, 1728, 1264; HRMS calcd for $\mathrm{C}_{22} \mathrm{H}_{22} \mathrm{O}_{5}$ 366.1467, found 366.1472. 
Diethyl 2-(4-methoxyphenyl)-1H-indene-1,1-dicarboxylate (43). The indicated compound was obtained as a pale yellow oil in a 96\% yield: ${ }^{1} \mathrm{H}$ NMR $\left(\mathrm{CDCl}_{3}\right) \delta$ 1.08 (t, $J=7.2 \mathrm{~Hz}, 6 \mathrm{H}), 3.80(\mathrm{~s}, 3 \mathrm{H}), 4.12(\mathrm{~m}, 4 \mathrm{H}), 6.87$ (dt, $J=2.0,9.2 \mathrm{~Hz}, 2 \mathrm{H})$, 7.18 (s, 1H), 7.21 (m, 1H), 7.32 (m, 2H), 7.55 (dt, $J=2.0,8.8 \mathrm{~Hz}, 2 \mathrm{H}), 7.62$ (d, $J=$ 7.6 Hz, 1H); ${ }^{13} \mathrm{C} \mathrm{NMR}\left(\mathrm{CDCl}_{3}\right) \delta 13.9,55.3,62.0,70.9,113.6,121.3,124.1,125.9$, 126.7, 128.7, 128.9, 129.6, 141.9, 144.0, 145.3, 159.5, 168.5; IR $\left(\mathrm{CHCl}_{3}, \mathrm{~cm}^{-1}\right) 3068$, 2981, 1734, 1253; HRMS calcd for $\mathrm{C}_{22} \mathrm{H}_{22} \mathrm{O}_{5}$ 366.1467, found 366.1473.

Diethyl (2-ethynylphenyl)malonate (44). The indicated compound was obtained as a pale yellow oil in an 81\% yield: ${ }^{1} \mathrm{H}$ NMR $\left(\mathrm{CDCl}_{3}\right) \delta 1.27(\mathrm{t}, J=7.2 \mathrm{~Hz}, 6 \mathrm{H}), 3.32$ (s, 1H), 4.25 (m, 4H), 5.32 (s, 1H), 7.29 (dt, $J=1.2,7.6 \mathrm{~Hz}, 1 \mathrm{H}), 7.38$ (dt, $J=1.6,7.6$ $\mathrm{Hz}, 1 \mathrm{H}), 7.52(\mathrm{dt}, J=1.2,8.0 \mathrm{~Hz}, 2 \mathrm{H}) ;{ }^{13} \mathrm{C} \mathrm{NMR}\left(\mathrm{CDCl}_{3}\right) \delta 14.1,55.5,61.9,81.2$, 82.3, 122.6, 128.0, 128.8, 129.2, 132.8, 135.3, 168.0; IR $\left(\mathrm{CHCl}_{3}, \mathrm{~cm}^{-1}\right)$ 3297, 3053, 2983, 1749, 1733, 1265; HRMS calcd for $\mathrm{C}_{15} \mathrm{H}_{16} \mathrm{O}_{4} 260.1049$, found 260.1052 .

Ethyl cyano(2-iodophenyl)acetate (45). The indicated compound was obtained as a yellow oil in an 82\% yield: ${ }^{1} \mathrm{H}$ NMR $\left(\mathrm{CDCl}_{3}\right) \delta 1.31(\mathrm{t}, J=7.2 \mathrm{~Hz}, 3 \mathrm{H}), 4.30(\mathrm{~m}$, 2H), 5.19 (s, 1H), 7.10 (dt, $J=1.5,7.8 \mathrm{~Hz}, 1 \mathrm{H}$ ), 7.44 (dt, $J=1.2,7.5 \mathrm{~Hz}, 1 \mathrm{H}), 7.60$ (dd, $J=1.5,7.8 \mathrm{~Hz}, 1 \mathrm{H}), 7.90(\mathrm{dd}, J=1.2,7.8 \mathrm{~Hz}, 1 \mathrm{H}) ;{ }^{13} \mathrm{C} \mathrm{NMR}\left(\mathrm{CDCl}_{3}\right) \delta 14.2$, 48.3, 63.8, 100.0, 115.6, 129.5, 129.6, 131.1, 134.1, 140.4, 164.3; IR $\left(\mathrm{CHCl}_{3}, \mathrm{~cm}^{-1}\right)$ 3070, 2957, 1743, 1466, 1217; HRMS calcd for $\mathrm{C}_{11} \mathrm{H}_{10} \mathrm{INO}_{2}$ 314.9756, found 314.9760.

Ethyl cyano[2-(oct-1-ynyl)phenyl]acetate (46). The indicated compound was obtained as a pale yellow oil in a 92\% yield: ${ }^{1} \mathrm{H} \mathrm{NMR}\left(\mathrm{CDCl}_{3}\right) \delta 0.91(\mathrm{t}, J=6.9 \mathrm{~Hz}$, 3H), 1.26-1.37 (m, 7H), 1.45 (m, 2H), 1.60 (m, 2H), 2.44 (t, J = 7.2 Hz, 2H), 4.25 (m, 2H), 5.28 (s, 1H), 7.29-7.37 (m, 2H), 7.46 (m, 1H), 7.50 (m, 1H); ${ }^{13} \mathrm{C}$ NMR $\left(\mathrm{CDCl}_{3}\right)$ $\delta 14.2$, 14.3, 19.8, 22.8, 28.7, 28.9, 31.6, 42.0, 63.4, 77.7, 97.8, 115.9, 124.3, 128.3, 128.7, 129.2, 132.0, 132.8, 165.0; IR $\left(\mathrm{CHCl}_{3}, \mathrm{~cm}^{-1}\right)$ 3054, 2933, 2226, 1747, 1265; HRMS calcd for $\mathrm{C}_{19} \mathrm{H}_{23} \mathrm{NO}_{2}$ 297.1729, found 297.1733. 
Ethyl 1-cyano-2-n-hexyl-1H-indene-1-carboxylate (47). The indicated compound was obtained as a pale yellow oil in an 83\% yield: ${ }^{1} \mathrm{H}$ NMR $\left(\mathrm{CDCl}_{3}\right) \delta 0.92(\mathrm{t}, J=$ $7.2 \mathrm{~Hz}, 3 \mathrm{H}), 1.27-1.47$ (m, 9H), 1.74 (m, 2H), 2.49 (m, 2H), 4.28 (m, 2H), 6.71 (s, 1H), 7.26 (t, $J=7.6 \mathrm{~Hz}, 1 \mathrm{H}), 7.30$ (d, $J=7.6 \mathrm{~Hz}, 1 \mathrm{H}), 7.38$ (d, $J=7.6 \mathrm{~Hz}, 1 \mathrm{H}), 7.57$ (d, $J=7.6 \mathrm{~Hz}, 1 \mathrm{H}) ;{ }^{13} \mathrm{C} \mathrm{NMR}\left(\mathrm{CDCl}_{3}\right) \delta 14.0,14.1,22.6,27.8,28.2,29.1,31.7,58.5$, 63.5, 116.4, 121.5, 123.2, 126.4, 129.9, 130.8, 139.3, 144.0, 146.1, 165.4; IR $\left(\mathrm{CHCl}_{3}\right.$, $\mathrm{cm}^{-1}$ ) 3020, 2930, 2246, 1743, 1217; HRMS calcd for $\mathrm{C}_{19} \mathrm{H}_{23} \mathrm{NO}_{2}$ 297.1729, found 297.1733.

Ethyl cyano[2-(cyclohexylethynyl)phenyl]acetate (48). The indicated compound was obtained as a pale yellow oil in a 76\% yield: ${ }^{1} \mathrm{H}$ NMR $\left(\mathrm{CDCl}_{3}\right) \delta 1.28(\mathrm{t}, J=7.2$ Hz, 3H), 1.39 (m, 3H), 1.57 (m, 3H), 1.74 (m, 2H), 1.90 (m, 2H), 2.64 (m, 1H), 4.26 (m, 2H), 5.27 (s, 1H), 7.33 (m, 2H), $7.46(\mathrm{~m}, 1 \mathrm{H}), 7.50(\mathrm{~m}, 1 \mathrm{H}) ;{ }^{13} \mathrm{C} \mathrm{NMR}\left(\mathrm{CDCl}_{3}\right) \delta$ 14.0, 25.0, 25.9, 29.8, 32.5, 41.9, 63.2, 77.2, 101.5, 115.8, 124.1, 128.1, 128.4, 129.0, 131.7, 132.5, 164.8; IR $\left(\mathrm{CHCl}_{3}, \mathrm{~cm}^{-1}\right)$ 3020, 2933, 2228, 1748, 1216; HRMS calcd for $\mathrm{C}_{19} \mathrm{H}_{21} \mathrm{NO}_{2}$ 295.1572, found 295.1577.

Ethyl 1-cyano-2-cyclohexyl-1H-indene-1-carboxylate (49). The indicated compound was obtained as a pale yellow oil in a $78 \%$ yield: ${ }^{1} \mathrm{H}$ NMR $\left(\mathrm{CDCl}_{3}\right) \delta$ 1.25-1.44 (m, 8H), 1.72-1.94 (m, 4H), 2.21 (m, 1H), 2.44 (m, 1H), 4.24 (m, 2H), 6.74 (m, 1H), 7.24 (dt, $J=1.5,7.5 \mathrm{~Hz}, 1 \mathrm{H}$ ), 7.29 (d, $J=6.9 \mathrm{~Hz}, 1 \mathrm{H}$ ), 7.36 (dt, $J=1.2,7.5$ $\mathrm{Hz}, 1 \mathrm{H}), 7.52$ (d, $J=7.8 \mathrm{~Hz}, 1 \mathrm{H}) ;{ }^{13} \mathrm{C} \mathrm{NMR}\left(\mathrm{CDCl}_{3}\right) \delta 14.1,26.2,26.6,26.7,33.1$, 33.7, 38.6, 58.2, 63.6, 116.7, 121.8, 123.1, 126.7, 130.0, 130.8, 139.5, 144.1, 151.3, 165.9; IR $\left(\mathrm{CHCl}_{3}, \mathrm{~cm}^{-1}\right)$ 3020, 2932, 2250, 1710, 1217; HRMS calcd for $\mathrm{C}_{19} \mathrm{H}_{21} \mathrm{NO}_{2}$ 295.1572, found 295.1577.

Ethyl 1-cyano-2-phenyl-1H-indene-1-carboxylate (50). The indicated compound was obtained as a pale yellow oil in an $86 \%$ yield: ${ }^{1} \mathrm{H}$ NMR $\left(\mathrm{CDCl}_{3}\right) \delta 1.16(\mathrm{t}, J=$ $7.2 \mathrm{~Hz}, 3 \mathrm{H}), 4.24$ (m, 2H), 7.36 (m, 2H), 7.45 (m, 5H), 7.69 (m, 3H); ${ }^{13} \mathrm{C}$ NMR 
$\left(\mathrm{CDCl}_{3}\right) \delta 13.8,56.7,63.7,116.6,122.5,123.0,126.0,127.5,129.0,129.1,130.3$ 131.1, 131.9, 140.0, 142.9, 143.3, 165.7; IR $\left(\mathrm{CHCl}_{3}, \mathrm{~cm}^{-1}\right)$ 3020, 2984, 2250, 1740, 1217; HRMS calcd for $\mathrm{C}_{19} \mathrm{H}_{15} \mathrm{NO}_{2} 289.1103$, found 289.1108 .

Ethyl (2-iodophenyl)(phenylsulfonyl)acetate (51). The indicated compound was obtained as a white solid in a 78\% yield: $\operatorname{mp} 118-119{ }^{\circ} \mathrm{C} ;{ }^{1} \mathrm{H}$ NMR $\left(\mathrm{CDCl}_{3}\right) \delta 1.15$ (t, $J=7.2 \mathrm{~Hz}, 3 \mathrm{H}), 4.15$ (m, 2H), 5.73 (s, 1H), 7.00 (dt, $J=1.6,7.8 \mathrm{~Hz}, 1 \mathrm{H}), 7.33$ (t, $J$ $=7.5 \mathrm{~Hz}, 1 \mathrm{H}), 7.41$ (t, $J=7.6 \mathrm{~Hz}, 2 \mathrm{H}), 7.58$ (t, $J=7.2 \mathrm{~Hz}, 1 \mathrm{H}), 7.65$ (d, $J=7.6 \mathrm{~Hz}$, 2H), $7.71(\mathrm{t}, J=8.0 \mathrm{~Hz}, 1 \mathrm{H}), 7.97(\mathrm{dd}, J=1.5,7.8 \mathrm{~Hz}, 1 \mathrm{H}) ;{ }^{13} \mathrm{C} \mathrm{NMR}\left(\mathrm{CDCl}_{3}\right) \delta$ 14.0, 62.9, 103.8, 128.6, 129.1, 129.5, 131.1, 131.2, 134.5, 137.5, 140.0, 164.1; IR $\left(\mathrm{CHCl}_{3}, \mathrm{~cm}^{-1}\right)$ 3068, 2984, 1741, 1328, 1157; HRMS calcd for $\mathrm{C}_{16} \mathrm{H}_{15} \mathrm{ISO}_{4}$ 329.9736, found 329.9742 .

Ethyl [2-(phenylethynyl)phenyl](phenylsulfonyl)acetate (52). The indicated compound was obtained as a pale yellow oil in a $91 \%$ yield: ${ }^{1} \mathrm{H}$ NMR $\left(\mathrm{CDCl}_{3}\right) \delta$ 1.27 (t, $J=7.2 \mathrm{~Hz}, 3 \mathrm{H}), 4.27$ (m, 2H), 6.00 (s, 1H), 7.33-7.39 (m, 7H), 7.42-7.46 (m, 3H), 7.55 (m, 1H), 7.65 (dd, $J=1.2,7.2 \mathrm{~Hz}, 2 \mathrm{H}), 7.99$ (dd, $J=1.6,7.2 \mathrm{~Hz}, 1 \mathrm{H}) ;{ }^{13} \mathrm{C}$ NMR $\left(\mathrm{CDCl}_{3}\right) \delta 14.0,62.8,71.6,86.1,94.4,122.5,125.1,128.5,128.6,128.7,128.9$, 129.4, 129.4, 129.5, 130.1, 131.6, 132.1, 134.1, 137.5, 164.4; IR $\left(\mathrm{CHCl}_{3}, \mathrm{~cm}^{-1}\right)$ 3056, 2985, 1740, 1265; HRMS calcd for $\mathrm{C}_{24} \mathrm{H}_{20} \mathrm{SO}_{4}$ 404.1082, found 404.1088.

Ethyl 2-phenyl-1-phenylsulfonyl-1H-indene-1-carboxylate (53). The indicated compound was obtained as a pale yellow oil in a $36 \%$ yield: ${ }^{1} \mathrm{H}$ NMR $\left(\mathrm{CDCl}_{3}\right) \delta$ 1.23 (t, $J=7.2 \mathrm{~Hz}, 3 \mathrm{H}), 4.35$ (m, 2H), 6.96 (s, 1H), 7.01 (dd, $J=1.2,8.4 \mathrm{~Hz}, 2 \mathrm{H}$ ), 7.09 (t, $J=8.0 \mathrm{~Hz}, 2 \mathrm{H}), 7.14$ (d, $J=6.8 \mathrm{~Hz}, 1 \mathrm{H}), 7.34-7.41$ (m, 6H), 7.51 (dt, $J=1.6$, $8.0 \mathrm{~Hz}, 2 \mathrm{H}), 7.96$ (d, $J=7.6 \mathrm{~Hz}, 1 \mathrm{H}) ;{ }^{13} \mathrm{C} \mathrm{NMR}\left(\mathrm{CDCl}_{3}\right) \delta 13.9,63.0,84.0,121.7$, 126.2, 126.7, 127.4, 127.9, 128.3, 128.5, 130.0, 130.2, 132.7, 133.7, 134.3, 135.4, 138.1, 142.9, 143.9, 165.8; IR $\left(\mathrm{CHCl}_{3}, \mathrm{~cm}^{-1}\right)$ 3062, 2982, 1718, 1195; HRMS calcd for $\mathrm{C}_{24} \mathrm{H}_{20} \mathrm{SO}_{4}$ 404.1082, found 404.1088. 
Ethyl (2-iodophenyl)acetate (54): The indicated compound was obtained as white solid in a 95\% yield: $\operatorname{mp} 125-126{ }^{\circ} \mathrm{C} ;{ }^{1} \mathrm{H} \mathrm{NMR}\left(\mathrm{CDCl}_{3}\right) \delta 1.26(\mathrm{t}, J=7.2 \mathrm{~Hz}, 3 \mathrm{H})$, 3.77 (s, 2H), 4.17 (q, $J=7.2 \mathrm{~Hz}, 2 \mathrm{H}), 6.94$ (m, 1H), 7.28 (m, 2H), 7.82 (d, $J=8.0 \mathrm{~Hz}$, $1 \mathrm{H})$.

Ethyl [2-(phenylethynyl)phenyl]acetate (55). The indicated compound was obtained as a pale yellow oil in a 98\% yield: ${ }^{1} \mathrm{H} \mathrm{NMR}\left(\mathrm{CDCl}_{3}\right) \delta 1.21(\mathrm{t}, J=7.2 \mathrm{~Hz}$, 3H), 3.90 (s, 2H), 4.15 (q, $J=7.2 \mathrm{~Hz}, 2 \mathrm{H}), 7.26-7.34$ (m, 6H), 7.52-7.55 (m, 3H); ${ }^{13} \mathrm{C}$ NMR $\left(\mathrm{CDCl}_{3}\right) \delta 14.2,40.4,61.0,87.5,94.0,123.3,123.6,127.2,128.4,128.5,128.6$, 130.0, 131.6, 132.1, 136.5, 171.3; IR $\left(\mathrm{CHCl}_{3}, \mathrm{~cm}^{-1}\right)$ 3056, 2982, 1733, 1265; HRMS calcd for $\mathrm{C}_{18} \mathrm{H}_{16} \mathrm{O}_{2} 264.1150$, found 264.1156.

(2-Iodobenzyl)(phenyl)sulfone (56). The indicated compound was obtained as a bright colorless solid in a 63\% yield: $\mathrm{mp} 131-132{ }^{\circ} \mathrm{C} ;{ }^{1} \mathrm{H}$ NMR $\left(\mathrm{CDCl}_{3}\right) \delta 4.59$ (s, 2H), 7.00 (dt, $J=1.5,7.8 \mathrm{~Hz}, 1 \mathrm{H}), 7.44-7.53$ (m, 3H), 7.60-7.66 (m, 3H), 7.73 (dd, $J$ $=0.9,7.8 \mathrm{~Hz}, 1 \mathrm{H})$.

1-Phenylethynyl-2-(phenylsulfonylmethyl)benzene (57). The indicated compound was obtained as a pale yellow oil in a 99\% yield: ${ }^{1} \mathrm{H}$ NMR $\left(\mathrm{CDCl}_{3}\right) \delta 4.68(\mathrm{~s}, 2 \mathrm{H})$, 7.26-7.37 (m, 10H), 7.48 (t, $J=7.2 \mathrm{~Hz}, 2 \mathrm{H}), 7.58$ (d, $J=8.4 \mathrm{~Hz}, 2 \mathrm{H}) ;{ }^{13} \mathrm{C} \mathrm{NMR}$ $\left(\mathrm{CDCl}_{3}\right) \delta$ 60.8, 86.5, 93.7, 122.6, 124.7, 128.5, 128.6, 128.7, 128.8, 128.9, 129.9, 131.4, 131.6, 132.2, 133.7, 138.1; IR $\left(\mathrm{CHCl}_{3}, \mathrm{~cm}^{-1}\right)$ 3054, 2926, 1713, 1265; HRMS calcd for $\mathrm{C}_{21} \mathrm{H}_{16} \mathrm{SO}_{2} 332.0871$, found 332.0877 .

\section{Diethyl 3-[4-(ethoxycarbonyl)phenyl]-2-phenyl-1H-indene-1,1-dicarboxylate}

(58). The indicated compound was obtained as a pale yellow oil in an $86 \%$ yield: ${ }^{1} \mathrm{H}$ NMR $\left(\mathrm{CDCl}_{3}\right) \delta 1.09$ (t, $\left.J=7.2 \mathrm{~Hz}, 6 \mathrm{H}\right), 1.37$ (t, $\left.J=7.2 \mathrm{~Hz}, 3 \mathrm{H}\right), 4.14(\mathrm{~m}, 4 \mathrm{H})$, 4.35 (q, $J=7.2 \mathrm{~Hz}, 2 \mathrm{H}), 7.17$ (m, 5H), 7.25 (m, 1H), 7.34 (m, 2H), 7.39 (d, $J=8.4$ $\mathrm{Hz}, 2 \mathrm{H}), 7.69$ (dd, $J=1.2,7.6 \mathrm{~Hz}, 1 \mathrm{H}), 8.00$ (d, $J=8.4 \mathrm{~Hz}, 2 \mathrm{H}) ;{ }^{13} \mathrm{C} \mathrm{NMR}\left(\mathrm{CDCl}_{3}\right) \delta$ 13.8, 14.4, 61.1, 62.1, 73.0, 121.1, 124.8, 126.9, 127.6, 127.7, 128.8, 129.6, 129.7, 
130.3, 134.7, 139.1, 140.9, 141.8, 144.0, 144.4, 166.4, 168.0; IR $\left(\mathrm{CHCl}_{3}, \mathrm{~cm}^{-1}\right)$ 3065, 2982, 1718, 1275; HRMS calcd for $\mathrm{C}_{30} \mathrm{H}_{28} \mathrm{O}_{6}$ 484.1886, found 484.1895 .

\section{Diethyl 3-[3-(ethoxycarbonyl)phenyl]-2-phenyl-1H-indene-1,1-dicarboxylate}

(59). The indicated compound was obtained as a pale yellow oil in a $74 \%$ yield: ${ }^{1} \mathrm{H}$ NMR $\left(\mathrm{CDCl}_{3}\right) \delta 1.09(\mathrm{t}, J=7.2 \mathrm{~Hz}, 6 \mathrm{H}), 1.37$ (t, $\left.J=7.2 \mathrm{~Hz}, 3 \mathrm{H}\right), 4.14(\mathrm{~m}, 4 \mathrm{H})$, 4.35 (q, $J=7.2 \mathrm{~Hz}, 2 \mathrm{H}), 7.16-7.28$ (m, 6H), 7.34 (m, 3H), 7.44 (m, 1H), 7.71 (d, $J=$ $7.2 \mathrm{~Hz}, 1 \mathrm{H}), 7.98(\mathrm{dt}, J=1.6,7.6 \mathrm{~Hz}, 1 \mathrm{H}), 8.10(\mathrm{t}, J=1.2 \mathrm{~Hz}, 1 \mathrm{H}) ;{ }^{13} \mathrm{C} \mathrm{NMR}$ $\left(\mathrm{CDCl}_{3}\right) \delta 13.8,14.4,61.1,62.1,73.0,121.1,124.8,126.9,127.5,127.7,128.6,128.8$, 129.0, 130.3, 130.5, 130.8, 134.2, 134.6, 134.7, 140.9, 141.5, 144.0, 144.7, 166.4, 168.1; IR $\left(\mathrm{CHCl}_{3}, \mathrm{~cm}^{-1}\right)$ 3067, 2982, 1730, 1257; HRMS calcd for $\mathrm{C}_{30} \mathrm{H}_{28} \mathrm{O}_{6}$ 484.1886, found 484.1892 .

\section{Diethyl 3-[2-(ethoxycarbonyl)phenyl]-2-phenyl-1H-indene-1,1-dicarboxylate}

(60). The indicated compound was obtained as a pale yellow oil in a 78\% yield: ${ }^{1} \mathrm{H}$ NMR $\left(\mathrm{CDCl}_{3}\right) \delta 0.82(\mathrm{t}, J=7.2 \mathrm{~Hz}, 3 \mathrm{H}), 0.95(\mathrm{t}, J=7.2 \mathrm{~Hz}, 3 \mathrm{H}), 1.24(\mathrm{t}, J=7.2$ Hz, 3H), 4.04 (m, 4H), 4.28 (m, 2H), 6.90 (m, 1H), 7.15 (m, 4H), 7.27 (m, 4H), 7.37 (m, 2H), 7.67 (m, 1H), 8.05 (m, 1H); ${ }^{13} \mathrm{C}$ NMR $\left(\mathrm{CDCl}_{3}\right) \delta 13.5,13.8,14.2,61.5,61.8$, 62.3, 72.8, 120.6, 124.7, 126.6, 127.4, 127.7, 128.1, 128.9, 130.2, 130.8, 131.8, 131.9, 132.6, 135.2, 135.6, 139.6, 140.4, 146.0, 146.1, 167.7, 167.8, 169.1; IR $\left(\mathrm{CHCl}_{3}, \mathrm{~cm}^{-1}\right)$ 3019, 2982, 1725, 1702, 1217; HRMS calcd for $\mathrm{C}_{30} \mathrm{H}_{28} \mathrm{O}_{6}$ 484.1886, found 484.1892.

Diethyl 3-(4-nitrophenyl)-2-phenyl-1H-indene-1,1-dicarboxylate (61). The indicated compound was obtained as a pale yellow oil in an 83\% yield: ${ }^{1} \mathrm{H}$ NMR $\left(\mathrm{CDCl}_{3}\right) \delta 1.08(\mathrm{t}, J=7.2 \mathrm{~Hz}, 6 \mathrm{H}), 4.15(\mathrm{~m}, 4 \mathrm{H}), 7.23(\mathrm{~m}, 6 \mathrm{H}), 7.36(\mathrm{~m}, 2 \mathrm{H}), 7.49$ (d, $J=8.8 \mathrm{~Hz}, 2 \mathrm{H}), 7.71(\mathrm{~d}, J=7.6 \mathrm{~Hz}, 1 \mathrm{H}), 8.16(\mathrm{~d}, J=8.8 \mathrm{~Hz}, 2 \mathrm{H}) ;{ }^{13} \mathrm{C} \mathrm{NMR}\left(\mathrm{CDCl}_{3}\right)$ $\delta$ 13.8, 62.2, 73.2, 120.8, 123.8, 125.0, 127.2, 127.9, 128.0, 129.0, 130.2, 130.6, 134.3, 140.9, 141.4, 142.9, 143.1, 143.8, 147.2, 167.7; IR $\left(\mathrm{CHCl}_{3}, \mathrm{~cm}^{-1}\right)$ 3024, 2985, 1716, 1235; HRMS calcd for $\mathrm{C}_{27} \mathrm{H}_{23} \mathrm{NO}_{6} 457.1525$, found 457.1531 . 
Diethyl 3-(3-nitrophenyl)-2-phenyl-1H-indene-1,1-dicarboxylate (62). The indicated compound was obtained as a pale yellow oil in an $82 \%$ yield: ${ }^{1} \mathrm{H}$ NMR $\left(\mathrm{CDCl}_{3}\right) \delta 1.09(\mathrm{t}, J=7.2 \mathrm{~Hz}, 6 \mathrm{H}), 4.15(\mathrm{~m}, 4 \mathrm{H}), 7.23(\mathrm{~m}, 6 \mathrm{H}), 7.36(\mathrm{~m}, 2 \mathrm{H}), 7.46(\mathrm{~d}$, $J=8.0 \mathrm{~Hz}, 1 \mathrm{H}), 7.60$ (d, $J=7.6 \mathrm{~Hz}, 1 \mathrm{H}), 7.72$ (d, $J=7.2 \mathrm{~Hz}, 1 \mathrm{H}), 8.13$ (d, $J=8.4$ $\mathrm{Hz}, 1 \mathrm{H}), 8.24$ (s, $1 \mathrm{H}) ;{ }^{13} \mathrm{C} \mathrm{NMR}\left(\mathrm{CDCl}_{3}\right) \delta 13.8,62.2,73.1,120.7,122.8,124.4$, 125.0, 127.2, 127.9, 129.0, 129.6, 130.3, 134.2, 135.9, 136.1, 140.9, 142.6, 142.9, 143.9, 148.4, 167.7; IR $\left(\mathrm{CHCl}_{3}, \mathrm{~cm}^{-1}\right)$ 3019, 2983, 1727, 1715, 1219; HRMS calcd for $\mathrm{C}_{27} \mathrm{H}_{23} \mathrm{NO}_{6} 457.1525$, found 457.1531.

\section{Diethyl 2-phenyl-3-[4-(trifluoromethyl)phenyl]-1H-indene-1,1-dicarboxylate (63).}

The indicated compound was obtained as a pale yellow oil in an $85 \%$ yield: ${ }^{1} \mathrm{H}$ NMR $\left(\mathrm{CDCl}_{3}\right) \delta 1.09(\mathrm{t}, J=7.2 \mathrm{~Hz}, 6 \mathrm{H}), 4.15$ (m, 4H), $7.23(\mathrm{~m}, 6 \mathrm{H}), 7.35$ (m, 2H), 7.45 (d, $J=8.4 \mathrm{~Hz}, 2 \mathrm{H}), 7.58$ (d, $J=8.0 \mathrm{~Hz}, 2 \mathrm{H}), 7.72$ (d, $J=7.6 \mathrm{~Hz}, 1 \mathrm{H}) ;{ }^{13} \mathrm{C} \mathrm{NMR}$ $\left(\mathrm{CDCl}_{3}\right) \delta 13.8,62.1,73.1,121.0,122.8,124.9,125.5,127.0,127.7,127.8,128.9$, 130.0, 130.3, 134.6, 138.2, 140.9, 141.0, 142.1, 143.6, 144.4, 168.0; IR $\left(\mathrm{CHCl}_{3}, \mathrm{~cm}^{-1}\right)$ 3006, 2982, 1732, 1247; HRMS calcd for $\mathrm{C}_{28} \mathrm{H}_{23} \mathrm{~F}_{3} \mathrm{O}_{4} 480.1548$, found 480.1555 .

Diethyl 2-phenyl-3-[3-(trifluoromethyl)phenyl]-1H-indene-1,1-dicarboxylate (64).

The indicated compound was obtained as a pale yellow oil in a 91\% yield: ${ }^{1} \mathrm{H}$ NMR $\left(\mathrm{CDCl}_{3}\right) \delta 1.09(\mathrm{t}, J=7.2 \mathrm{~Hz}, 6 \mathrm{H}), 4.15$ (m, 4H), 7.23 (m, 6H), 7.37 (m, 3H), 7.49 (d, $J=7.6 \mathrm{~Hz}, 1 \mathrm{H}), 7.54$ (d, $J=7.6 \mathrm{~Hz}, 1 \mathrm{H}), 7.63$ (s, 1H), 7.72 (d, $J=6.8 \mathrm{~Hz}, 1 \mathrm{H}) ;{ }^{13} \mathrm{C}$ NMR $\left(\mathrm{CDCl}_{3}\right) \delta 13.8,62.1,73.0,120.9,124.6,124.9,126.4,126.5,127.0,127.7$, 127.8, 128.9, 129.0, 130.3, 133.0, 134.5, 135.2, 141.0, 142.1, 143.5, 144.3, 168.0; IR $\left(\mathrm{CHCl}_{3}, \mathrm{~cm}^{-1}\right)$ 3057, 2983, 1729, 1266; HRMS calcd for $\mathrm{C}_{28} \mathrm{H}_{23} \mathrm{~F}_{3} \mathrm{O}_{4} 480.1548$, found 480.1556.

Diethyl 2-phenyl-3-[2-(trifluoromethyl)phenyl]-1H-indene-1,1-dicarboxylate (65).

The indicated compound was obtained as a pale yellow oil in a $53 \%$ yield: ${ }^{1} \mathrm{H}$ NMR $\left(\mathrm{CDCl}_{3}\right) \delta 1.07(\mathrm{~m}, 6 \mathrm{H}), 4.13(\mathrm{~m}, 4 \mathrm{H}), 6.82(\mathrm{~m}, 1 \mathrm{H}), 7.11-7.17(\mathrm{~m}, 5 \mathrm{H}), 7.28(\mathrm{~m}, 2 \mathrm{H})$, 7.37 (d, $J=7.6 \mathrm{~Hz}, 1 \mathrm{H}), 7.47$ (t, $J=7.6 \mathrm{~Hz}, 1 \mathrm{H}), 7.54$ (t, $J=7.6 \mathrm{~Hz}, 1 \mathrm{H}), 7.66-7.73$ 
(m, 2H); ${ }^{13} \mathrm{C} \mathrm{NMR}\left(\mathrm{CDCl}_{3}\right) \delta 13.6,13.8,61.9,62.0,72.4,121.1,124.0,126.7,126.8$, 126.9, 127.4, 127.5, 128.2, 128.7, 129.4, 131.9, 132.1, 133.6, 134.3, 140.3, 141.8, 142.5, 146.4, 168.0, 168.2; IR $\left(\mathrm{CHCl}_{3}, \mathrm{~cm}^{-1}\right)$ 3067, 2982, 1734, 1717, 1220; HRMS calcd for $\mathrm{C}_{28} \mathrm{H}_{23} \mathrm{~F}_{3} \mathrm{O}_{4} 480.1548$, found 480.1556 .

Diethyl 3-(4-acetylphenyl)-2-phenyl-1H-indene-1,1-dicarboxylate (66). The indicated compound was obtained as a pale yellow oil in a 94\% yield: ${ }^{1} \mathrm{H}$ NMR $\left(\mathrm{CDCl}_{3}\right) \delta 1.09(\mathrm{t}, J=7.2 \mathrm{~Hz}, 6 \mathrm{H}), 2.59$ (s, 3H), 4.14 (m, 4H), 7.20 (m, 5H), 7.28 (m, 1H), 7.35 (m, 2H), 7.43 (dt, $J=1.8,8.7 \mathrm{~Hz}, 2 \mathrm{H}), 7.70$ (m, 1H), 7.92 (dt, $J=8.7 \mathrm{~Hz}$, $2 \mathrm{H}) ;{ }^{13} \mathrm{C} \mathrm{NMR}\left(\mathrm{CDCl}_{3}\right) \delta 13.8,26.7,62.1,73.0,121.0,124.8,126.9,127.6,127.7$, 128.5, 128.8, 129.9, 130.3, 134.7, 136.3, 139.5, 141.0, 142.0, 143.9, 144.4, 168.0, 197.7; IR $\left(\mathrm{CHCl}_{3}, \mathrm{~cm}^{-1}\right)$ 2980, 1733, 1685, 1221; HRMS calcd for $\mathrm{C}_{29} \mathrm{H}_{26} \mathrm{O}_{5}$ 454.1780, found 454.1786 .

Diethyl 3-(4-chlorophenyl)-2-phenyl-1H-indene-1,1-dicarboxylate (67). The indicated compound was obtained as a pale yellow oil in an $84 \%$ yield: ${ }^{1} \mathrm{H}$ NMR $\left(\mathrm{CDCl}_{3}\right) \delta 1.08(\mathrm{t}, J=7.2 \mathrm{~Hz}, 6 \mathrm{H}), 4.14(\mathrm{~m}, 4 \mathrm{H}), 7.17-7.37$ (m, 12H), 7.70 (d, $J=7.2$ $\mathrm{Hz}, 1 \mathrm{H}) ;{ }^{13} \mathrm{C} \mathrm{NMR}\left(\mathrm{CDCl}_{3}\right) \delta 14.0,62.2,73.1,121.2,125.0,127.1,127.7,127.9$, 129.0, 130.5, 130.6, 131.2, 133.0, 133.9, 135.0, 141.1, 141.4, 143.9, 144.8, 168.3; IR $\left(\mathrm{CHCl}_{3}, \mathrm{~cm}^{-1}\right)$ 3019, 2983, 1725, 1714, 1218; HRMS calcd for $\mathrm{C}_{27} \mathrm{H}_{23} \mathrm{ClO}_{4} 446.1285$, found 446.1292 .

Diethyl 2-phenyl-3-(thien-2-yl)-1H-indene-1,1-dicarboxylate (68). The indicated compound was obtained as a pale yellow oil in a 64\% yield: ${ }^{1} \mathrm{H}$ NMR $\left(\mathrm{CDCl}_{3}\right) \delta$ $1.08(\mathrm{t}, J=7.2 \mathrm{~Hz}, 6 \mathrm{H}), 4.14(\mathrm{~m}, 4 \mathrm{H}), 7.00$ (dd, $J=3.6,5.1 \mathrm{~Hz}, 1 \mathrm{H}), 7.13$ (dd, $J=$ 1.2, 3.6 Hz, 1H), 7.25-7.45 (m, 8H), 7.63 (d, $J=7.2 \mathrm{~Hz}, 1 \mathrm{H}), 7.69$ (d, $J=7.2 \mathrm{~Hz}$, 1H); ${ }^{13} \mathrm{C} \mathrm{NMR}\left(\mathrm{CDCl}_{3}\right) \delta 14.0,62.2,62.3,73.3,121.6,124.9,126.6,127.1,127.2$, 127.9, 128.0, 128.3, 130.0, 130.7, 135.3, 135.5, 138.1, 140.8, 141.4, 144.4, 168.2; IR $\left(\mathrm{CHCl}_{3}, \mathrm{~cm}^{-1}\right)$ 3001, 2984, 1714, 1363, 1223; HRMS calcd for $\mathrm{C}_{25} \mathrm{H}_{22} \mathrm{SO}_{4}$ 418.1239, found 418.1245 . 
Diethyl 2,3-diphenyl-1H-indene-1,1-dicarboxylate (69). The indicated compound was obtained as white crystals in a $72 \%$ yield: $\mathrm{mp} 85-87{ }^{\circ} \mathrm{C} ;{ }^{1} \mathrm{H}$ NMR $\left(\mathrm{CDCl}_{3}\right) \delta$ 1.07 (t, $J=7.2 \mathrm{~Hz}, 6 \mathrm{H}), 4.14$ (m, 4H), 7.15-7.36 (m, 13H), 7.69 (d, $J=7.2 \mathrm{~Hz}, 1 \mathrm{H}$ ); ${ }^{13} \mathrm{C} \mathrm{NMR}\left(\mathrm{CDCl}_{3}\right) \delta 13.8,61.9,72.8,121.3,124.6,126.7,127.3,127.6,127.8,128.5$, 128.7, 129.6, 130.3, 134.3, 135.1, 140.6, 141.0, 144.8, 145.1, 168.3; IR $\left(\mathrm{CHCl}_{3}, \mathrm{~cm}^{-1}\right)$ 3057, 3019, 2982, 1727, 1466, 1216; HRMS calcd for $\mathrm{C}_{27} \mathrm{H}_{24} \mathrm{O}_{4} 412.1675$, found 412.1680 .

Diethyl 3-(4-methylphenyl)-2-phenyl-1H-indene-1,1-dicarboxylate (70). The indicated compound was obtained as a pale yellow oil in a $69 \%$ yield: ${ }^{1} \mathrm{H}$ NMR $\left(\mathrm{CDCl}_{3}\right) \delta 1.08(\mathrm{t}, J=7.2 \mathrm{~Hz}, 6 \mathrm{H}), 2.34(\mathrm{~s}, 3 \mathrm{H}), 4.15(\mathrm{~m}, 4 \mathrm{H}), 7.12-7.35$ (m, 12H), $7.68(\mathrm{~d}, J=6.8 \mathrm{~Hz}, 1 \mathrm{H}) ;{ }^{13} \mathrm{C} \mathrm{NMR}\left(\mathrm{CDCl}_{3}\right) \delta 14.0,21.6,62.1,73.0,121.5,124.7$, 126.8, 127.4, 127.7, 127.8, 128.7, 128.9, 129.4, 129.7, 129.8, 130.5, 130.6, 135.5, 137.7, 141.2, 145.0, 145.4, 168.6; IR $\left(\mathrm{CHCl}_{3}, \mathrm{~cm}^{-1}\right)$ 3019, 2983, 1732, 1728, 1216; HRMS calcd for $\mathrm{C}_{28} \mathrm{H}_{26} \mathrm{O}_{4}$ 426.1831, found 426.1837; we have no good explanation as to why there are 18 aromatic signals in the ${ }^{13} \mathrm{C}$ NMR spectrum for compound.

Diethyl 3-(3-methylphenyl)-2-phenyl-1H-indene-1,1-dicarboxylate (71). The indicated compound was obtained as a pale yellow oil in a $74 \%$ yield: ${ }^{1} \mathrm{H}$ NMR $\left(\mathrm{CDCl}_{3}\right) \delta 1.08(\mathrm{t}, J=7.2 \mathrm{~Hz}, 6 \mathrm{H}), 2.30(\mathrm{~s}, 3 \mathrm{H}), 4.14(\mathrm{~m}, 4 \mathrm{H}), 7.09-7.36(\mathrm{~m}, 12 \mathrm{H})$, $7.70(\mathrm{~d}, J=7.8 \mathrm{~Hz}, 1 \mathrm{H}) ;{ }^{13} \mathrm{C} \mathrm{NMR}\left(\mathrm{CDCl}_{3}\right) \delta 14.0,21.7,62.1,72.9,121.5,124.7$, 126.8, 126.9, 127.4, 127.8, 128.5, 128.6, 128.7, 128.8, 129.8, 130.3, 130.5, 134.4, 138.2, 141.2, 145.1, 145.5, 168.5; IR $\left(\mathrm{CHCl}_{3}, \mathrm{~cm}^{-1}\right)$ 3021, 2983, 1728, 1218; HRMS calcd for $\mathrm{C}_{28} \mathrm{H}_{26} \mathrm{O}_{4} 426.1831$, found 426.1837 .

Diethyl 3-(2-methylphenyl)-2-phenyl-1H-indene-1,1-dicarboxylate (72). The indicated compound was obtained as a pale yellow oil in a $73 \%$ yield: ${ }^{1} \mathrm{H}$ NMR $\left(\mathrm{CDCl}_{3}\right) \delta 1.02(\mathrm{t}, J=7.2 \mathrm{~Hz}, 3 \mathrm{H}), 1.12(\mathrm{t}, J=7.2 \mathrm{~Hz}, 3 \mathrm{H}), 2.06$ (s, 3H), 4.12 (m, 4H), 6.97 (d, $J=8.0 \mathrm{~Hz}, 1 \mathrm{H}), 7.13$ (m, 3H), 7.19-7.31 (m, 8H), 7.69 (d, $J=8.0 \mathrm{~Hz}$, 
$1 \mathrm{H}) ;{ }^{13} \mathrm{C} \mathrm{NMR}\left(\mathrm{CDCl}_{3}\right) \delta 13.7,13.9,19.6,61.8,62.0,72.2,121.3,124.1,125.9$, 126.6, 127.3, 127.6, 128.0, 128.8, 129.1, 129.7, 130.5, 134.2, 135.0, 136.7, 140.8, 140.9, 144.5, 145.9, 168.0, 168.8; IR $\left(\mathrm{CHCl}_{3}, \mathrm{~cm}^{-1}\right)$ 3019, 2983, 1728, 1717, 1219; HRMS calcd for $\mathrm{C}_{28} \mathrm{H}_{26} \mathrm{O}_{4}$ 426.1831, found 426.1837.

Diethyl 3-(4-methoxyphenyl)-2-phenyl-1H-indene-1,1-dicarboxylate (73). The indicated compound was obtained as a pale yellow oil in a $14 \%$ yield: ${ }^{1} \mathrm{H}$ NMR $\left(\mathrm{CDCl}_{3}\right) \delta 1.08(\mathrm{t}, J=7.2 \mathrm{~Hz}, 6 \mathrm{H}), 3.80(\mathrm{~s}, 3 \mathrm{H}), 4.14(\mathrm{~m}, 4 \mathrm{H}), 6.85$ (d, $J=8.8 \mathrm{~Hz}$, 2H), 7.17-7.36 (m, 10H), 7.68 (d, $J=7.2 \mathrm{~Hz}, 1 \mathrm{H}) ;{ }^{13} \mathrm{C}$ NMR $\left(\mathrm{CDCl}_{3}\right) \delta$ 13.8, 55.2, 61.9, 72.7, 113.9, 121.2, 124.6, 126.5, 126.6, 127.1, 127.6, 128.7, 130.4, 130.8, 135.3, 139.9, 141.0, 144.4, 145.3, 159.1, 168.4; IR $\left(\mathrm{CHCl}_{3}, \mathrm{~cm}^{-1}\right)$ 2980, 1732, 1510, 1247; HRMS calcd for $\mathrm{C}_{28} \mathrm{H}_{26} \mathrm{O}_{5} 442.1780$, found 442.1785 .

Diethyl 2-allyl-2-[(2-phenylethynyl)phenyl]malonate (75). The indicated compound was obtained as a pale yellow oil in a $86 \%$ yield: ${ }^{1} \mathrm{H}$ NMR $\left(\mathrm{CDCl}_{3}\right) \delta$ 1.21 (t, $J=7.2 \mathrm{~Hz}, 6 \mathrm{H}$ ), 3.31 (d, $J=7.2 \mathrm{~Hz}, 2 \mathrm{H}$ ), 4.16-4.30 (m, 4H), 4.92 (d, $J=10.2$ Hz, 1H), 5.00 (d, $J=17.1 \mathrm{~Hz}, 1 \mathrm{H}), 5.81-5.90$ (m, 1H), 7.23-7.37 (m, 6H), 7.54-7.58 (m, 3H); ${ }^{13} \mathrm{C} \mathrm{NMR}\left(\mathrm{CDCl}_{3}\right) \delta 14.0,39.7,61.9,64.1,88.4,95.0,118.0,122.9,123.2$, 127.2, 127.9, 128.2, 128.5, 128.6, 131.3, 133.9, 134.2, 139.5, 170.2; IR $\left(\mathrm{CHCl}_{3}, \mathrm{~cm}^{-1}\right)$ 3056, 2937, 1742, 1717, 1266; HRMS calcd for $\mathrm{C}_{24} \mathrm{H}_{24} \mathrm{O}_{4}$ 376.1675, found 376.1683.

Diethyl 3-[4-(ethoxycarbonyl)phenyl]-2-hexyl-1H-indene-1,1-dicarboxylate (77). The indicated compound was obtained as a pale yellow oil in a $61 \%$ yield: ${ }^{1} \mathrm{H}$ NMR $\left(\mathrm{CDCl}_{3}\right) \delta 0.78(\mathrm{t}, J=7.2 \mathrm{~Hz}, 3 \mathrm{H}), 1.12(\mathrm{~m}, 6 \mathrm{H}), 1.26(\mathrm{t}, J=7.2 \mathrm{~Hz}, 8 \mathrm{H}), 1.40(\mathrm{t}, J=$ $7.2 \mathrm{~Hz}, 3 \mathrm{H}), 2.58$ (t, $J=8.0 \mathrm{~Hz}, 2 \mathrm{H}), 4.22$ (m, 4H), 4.39 (q, $J=7.2 \mathrm{~Hz}, 2 \mathrm{H}), 6.98$ (d, $J$ $=7.2 \mathrm{~Hz}, 1 \mathrm{H}), 7.23(\mathrm{~m}, 2 \mathrm{H}), 7.46(\mathrm{~d}, J=8.0 \mathrm{~Hz}, 2 \mathrm{H}), 7.62(\mathrm{~d}, J=7.2 \mathrm{~Hz}, 1 \mathrm{H}), 8.13$ (d, $J=8.0 \mathrm{~Hz}, 2 \mathrm{H}) ;{ }^{13} \mathrm{C} \mathrm{NMR}\left(\mathrm{CDCl}_{3}\right) \delta 14.0,14.1,14.4,22.6,27.6,29.1,29.7,31.4$, 61.1, 62.1, 71.9, 119.8, 124.9, 126.0, 128.6, 128.9, 129.8, 129.9, 139.6, 140.1, 142.6, 143.4, 145.4, 166.5, 168.4; IR $\left(\mathrm{CHCl}_{3}, \mathrm{~cm}^{-1}\right)$ 2981, 2930, 1730, 1273; HRMS calcd for $\mathrm{C}_{30} \mathrm{H}_{36} \mathrm{O}_{6}$ 492.2512, found 492.2521 . 
Diethyl 2-hexyl-3-(4-nitrophenyl)-1H-indene-1,1-dicarboxylate (78). The indicated compound was obtained as a pale yellow oil in a $63 \%$ yield: ${ }^{1} \mathrm{H}$ NMR $\left(\mathrm{CDCl}_{3}\right) \delta 0.78(\mathrm{t}, J=7.2 \mathrm{~Hz}, 3 \mathrm{H}), 1.14(\mathrm{~m}, 6 \mathrm{H}), 1.27(\mathrm{t}, J=7.2 \mathrm{~Hz}, 8 \mathrm{H}), 2.59(\mathrm{t}, J=$ $7.6 \mathrm{~Hz}, 2 \mathrm{H}), 4.23$ (m, 4H), 6.95 (d, $J=6.8 \mathrm{~Hz}, 1 \mathrm{H}), 7.26$ (m, 2H), 7.56 (d, $J=8.4 \mathrm{~Hz}$, 2H), 7.63 ( d, $J=6.8 \mathrm{~Hz}, 1 \mathrm{H}), 8.32$ (d, $J=8.4 \mathrm{~Hz}, 2 \mathrm{H}) ;{ }^{13} \mathrm{C} \mathrm{NMR}\left(\mathrm{CDCl}_{3}\right) \delta 14.0$, 22.5, 27.6, 29.2, 29.7, 31.4, 62.2, 72.0, 119.6, 124.0, 125.1, 126.3, 128.7, 129.9, 140.0, 141.5, 141.9, 144.6, 144.7, 147.4, 168.1; IR $\left(\mathrm{CHCl}_{3}, \mathrm{~cm}^{-1}\right)$ 2981, 2930, 1732, 1243; HRMS calcd for $\mathrm{C}_{27} \mathrm{H}_{31} \mathrm{NO}_{6} 465.2151$, found 465.2160 .

Diethyl 2-hexyl-3-(4-methoxyphenyl)-1H-indene-1,1-dicarboxylate (79). The indicated compound was obtained as a pale yellow oil in a 9\% yield: ${ }^{1} \mathrm{H}$ NMR $\left(\mathrm{CDCl}_{3}\right) \delta 0.82(\mathrm{t}, J=7.2 \mathrm{~Hz}, 3 \mathrm{H}), 1.13-1.32(\mathrm{~m}, 14 \mathrm{H}), 2.58$ (t, $\left.J=8.0 \mathrm{~Hz}, 2 \mathrm{H}\right), 3.87$ (s, 3H), 4.21 (m, 1H), 7.00 (d, $J=8.8 \mathrm{~Hz}, 2 \mathrm{H}), 7.06$ (d, $J=7.2 \mathrm{~Hz}, 1 \mathrm{H}), 7.21$ (t, $J=$ $7.2 \mathrm{~Hz}, 1 \mathrm{H}), 7.27$ (t, $J=7.2 \mathrm{~Hz}, 1 \mathrm{H}), 7.32$ (d, $J=8.4 \mathrm{~Hz}, 2 \mathrm{H}), 7.61$ (d, $J=7.2 \mathrm{~Hz}$, $1 \mathrm{H}) ;{ }^{13} \mathrm{C} \mathrm{NMR}\left(\mathrm{CDCl}_{3}\right) \delta 14.1,14.2,22.7,27.6,29.1,29.8,31.5,55.3,61.9,71.6$, 114.2, 120.0, 125.6, 126.9, 127.8, 128.4, 130.0, 140.1, 142.1, 143.1, 146.2, 159.1, 168.8; IR $\left(\mathrm{CHCl}_{3}, \mathrm{~cm}^{-1}\right)$ 3053, 2985, 2926, 1714, 1265; HRMS calcd for $\mathrm{C}_{28} \mathrm{H}_{34} \mathrm{O}_{5}$ 450.2406, found 450.2414 .

\section{Diethyl}

\section{2-(cyclohexen-1-yl)-3-[4-(ethoxycarbonyl)phenyl]-1H-indene-1,1-dicarboxylate}

(80). The indicated compound was obtained as a pale yellow oil in an $83 \%$ yield: ${ }^{1} \mathrm{H} \mathrm{NMR}\left(\mathrm{CDCl}_{3}\right) \delta 1.24(\mathrm{t}, J=7.2 \mathrm{~Hz}, 6 \mathrm{H}), 1.42(\mathrm{t}, J=7.2 \mathrm{~Hz}, 3 \mathrm{H}), 1.53(\mathrm{~m}, 4 \mathrm{H})$, 2.00 (m, 4H), 4.19 (m, 4H), 4.41 (t, $J=7.2 \mathrm{~Hz}, 2 \mathrm{H}), 5.60$ (s, 1H), 7.15 (d, $J=7.6 \mathrm{~Hz}$, 1H), 7.27 (m, 2H), 7.52 (d, $J=8.0 \mathrm{~Hz}, 2 \mathrm{H}), 7.60$ (d, $J=7.2 \mathrm{~Hz}, 1 \mathrm{H}), 8.10$ (d, $J=8.0$ $\mathrm{Hz}, 2 \mathrm{H}) ;{ }^{13} \mathrm{C} \mathrm{NMR}\left(\mathrm{CDCl}_{3}\right) \delta 14.0,14.4,21.8,22.9,25.9,29.1,61.1,61.9,72.0$, 120.5, 124.4, 126.3, 128.6, 129.1, 129.6, 129.7, 130.9, 132.4, 140.2, 140.7, 141.8, 144.8, 144.9, 166.6, 168.3; IR $\left(\mathrm{CHCl}_{3}, \mathrm{~cm}^{-1}\right)$ 3055, 2983, 2934, 1716, 1269; HRMS calcd for $\mathrm{C}_{30} \mathrm{H}_{32} \mathrm{O}_{6}$ 488.2199, found 488.2206. 
Diethyl 2-(cyclohexen-1-yl)-3-(4-nitrophenyl)-1H-indene-1,1-dicarboxylate (81).

The indicated compound was obtained as a pale yellow oil in an $87 \%$ yield: ${ }^{1} \mathrm{H}$ NMR $\left(\mathrm{CDCl}_{3}\right) \delta 1.26(\mathrm{t}, J=7.2 \mathrm{~Hz}, 6 \mathrm{H}), 1.54$ (m, 4H), 1.98 (m, 2H), 2.05 (m, 2H), 4.22 (m, 4H), 5.59 (s, 1H), 7.14 (m, 1H), 7.30 (m, 2H), 7.63 (m, 3H), 8.30 (m, 2H);

${ }^{13} \mathrm{C}$ NMR $\left(\mathrm{CDCl}_{3}\right) \delta 14.2,21.9,23.0,26.1,29.4,62.2,72.2,120.5,123.9,124.9$, 126.9, 127.8, 128.6, 128.9, 130.3, 131.9, 132.4, 140.9, 142.7, 144.2, 146.3, 147.3, 168.1; IR $\left(\mathrm{CHCl}_{3}, \mathrm{~cm}^{-1}\right)$ 3055, 2984, 1733, 1719, 1265; HRMS calcd for $\mathrm{C}_{27} \mathrm{H}_{27} \mathrm{NO}_{6}$ 461.1838, found 461.1846 .

\section{Diethyl 2-(cyclohexen-1-yl)-3-[4-(acetylphenyl)phenyl]-1H-indene-1,1-dicarbox-} ylate (82). The indicated compound was obtained as a pale yellow oil in a $78 \%$ yield: $\quad{ }^{1} \mathrm{H} \mathrm{NMR}\left(\mathrm{CDCl}_{3}\right) \delta 1.24(\mathrm{t}, J=7.2 \mathrm{~Hz}, 6 \mathrm{H}), 1.53(\mathrm{~m}, 4 \mathrm{H}), 2.00(\mathrm{~m}, 4 \mathrm{H}), 2.66$ (s, 3H), 4.22 (m, 4H), 5.60 (s, 1H), 7.16 (d, $J=7.6 \mathrm{~Hz}, 1 \mathrm{H}), 7.28$ (m, 2H), 7.55 (d, $J=$ $8.4 \mathrm{~Hz}, 2 \mathrm{H}), 7.60(\mathrm{~d}, J=7.2 \mathrm{~Hz}, 1 \mathrm{H}), 8.02(\mathrm{~d}, J=8.0 \mathrm{~Hz}, 2 \mathrm{H}) ;{ }^{13} \mathrm{C} \mathrm{NMR}\left(\mathrm{CDCl}_{3}\right) \delta$ 14.1, 21.8, 22.9, 25.9, 26.7, 29.1, 61.9, 72.0, 120.5, 124.4, 126.4, 128.4, 128.6, 129.4, 131.0, 132.4, 136.2, 140.6, 140.7, 141.7, 144.7, 145.0, 168.3, 197.9; IR $\left(\mathrm{CHCl}_{3}, \mathrm{~cm}^{-1}\right)$ 3056, 2983, 2934, 1739, 1716, 1268; HRMS calcd for $\mathrm{C}_{29} \mathrm{H}_{30} \mathrm{O}_{5}$ 458.2093, found 458.2101.

\section{Ethyl 1-cyano-3-[4-(ethoxycarbonyl)phenyl]-2-hexyl-1H-indene-1-carboxylate}

(83). The indicated compound was obtained as a pale yellow oil in a 53\% yield: ${ }^{1} \mathrm{H} \mathrm{NMR}\left(\mathrm{CDCl}_{3}\right) \delta 1.24(\mathrm{t}, J=7.2 \mathrm{~Hz}, 6 \mathrm{H}), 1.20-1.31(\mathrm{~m}, 9 \mathrm{H}), 1.43(\mathrm{t}, J=7.2 \mathrm{~Hz}$, 3H), 1.60 (m, 2H), 2.48 (m, 1H), $2.58(\mathrm{~m}, 1 \mathrm{H}), 4.28$ (m, 2H), 4.41 (q, J = $7.2 \mathrm{~Hz}, 2 \mathrm{H})$, 7.09 (d, $J=7.6 \mathrm{~Hz}, 1 \mathrm{H}), 7.34$ (m, 2H), 7.47 (d, $J=8.4 \mathrm{~Hz}, 2 \mathrm{H}), 7.61$ (d, $J=7.2 \mathrm{~Hz}$, $1 \mathrm{H}), 8.18(\mathrm{~d}, J=8.0 \mathrm{~Hz}, 2 \mathrm{H}) ;{ }^{13} \mathrm{C} \mathrm{NMR}\left(\mathrm{CDCl}_{3}\right) \delta 14.0,14.1,14.4,22.5,27.6,28.6$, 29.5, 31.3, 58.3, 61.2, 63.6, 116.4, 120.9, 123.2, 127.1, 128.8, 129.8, 130.0, 130.5, 138.0, 138.9, 140.8, 144.0, 144.7, 165.6, 166.3; IR $\left(\mathrm{CHCl}_{3}, \mathrm{~cm}^{-1}\right)$ 2983, 2930, 1741, 1715, 1266; HRMS calcd for $\mathrm{C}_{28} \mathrm{H}_{31} \mathrm{NO}_{4} 445.2253$, found 445.2261 . 


\section{Ethyl 3-[4-(ethoxycarbonyl)phenyl]-2-phenyl-1-phenylsulfonyl-1H-indene-1-car-}

boxylate (84). The indicated compound was obtained as a pale yellow oil in a $42 \%$ yield: $\quad{ }^{1} \mathrm{H} \mathrm{NMR}\left(\mathrm{CDCl}_{3}\right) \delta 1.25(\mathrm{t}, J=7.2 \mathrm{~Hz}, 3 \mathrm{H}), 1.37(\mathrm{t}, J=7.2 \mathrm{~Hz}, 3 \mathrm{H}), 4.35(\mathrm{~m}$, 4H), 6.91 (d, $J=8.4 \mathrm{~Hz}, 3 \mathrm{H}), 7.06$ (d, $J=7.2 \mathrm{~Hz}, 2 \mathrm{H}), 7.11-7.24$ (m, 7H), 7.37 (m, 2H), 7.47 (m, 1H), 7.93 (d, $J=8.4 \mathrm{~Hz}, 3 \mathrm{H}) ;{ }^{13} \mathrm{C} \mathrm{NMR}\left(\mathrm{CDCl}_{3}\right) \delta 13.9,14.4,61.2$, 63.0, 85.4, 121.1, 126.3, 127.3, 127.6, 127.8, 128.2, 129.0, 129.9, 130.0, 130.1, 130.2, 130.6, 132.4, 133.7, 135.7, 137.4, 137.8, 138.8, 145.2, 146.8, 165.8, 166.2; IR $\left(\mathrm{CHCl}_{3}, \mathrm{~cm}^{-1}\right)$ 2982, 1739, 1717, 1272; HRMS calcd for $\mathrm{C}_{33} \mathrm{H}_{28} \mathrm{SO}_{6} 552.1607$, found 552.1616.

Diethyl 13H-indeno[1,2-I]phenanthrene-13,13-dicarboxylate (85). The indicated compound was obtained as a pale yellow oil in a 35\% yield: ${ }^{1} \mathrm{H}$ NMR $\left(\mathrm{CDCl}_{3}\right) \delta$ $1.02(\mathrm{t}, J=7.2 \mathrm{~Hz}, 6 \mathrm{H}), 4.10(\mathrm{~m}, 4 \mathrm{H}), 7.41(\mathrm{t}, J=7.6 \mathrm{~Hz}, 1 \mathrm{H}), 7.61(\mathrm{~m}, 3 \mathrm{H}), 7.75(\mathrm{~m}$, 2H), 7.88 ( d, $J=7.6 \mathrm{~Hz}, 1 \mathrm{H}$ ), 8.21 (d, $J=8.0 \mathrm{~Hz}, 1 \mathrm{H}$ ), 8.40 (d, $J=7.6 \mathrm{~Hz}, 1 \mathrm{H}), 8.74$ (d, $J=7.6 \mathrm{~Hz}, 1 \mathrm{H}), 8.86(\mathrm{~m}, 2 \mathrm{H}) ;{ }^{13} \mathrm{C} \mathrm{NMR}\left(\mathrm{CDCl}_{3}\right) \delta 13.8,62.2,68.7,123.1,123.3$, 123.7, 124.8, 124.9, 126.5, 126.6, 126.8, 126.9, 127.0, 127.1, 128.5, 128.7, 129.1, 130.8, 131.9, 136.4, 137.5, 142.6, 142.9, 168.9; IR $\left(\mathrm{CHCl}_{3}, \mathrm{~cm}^{-1}\right)$ 3053, 2984, 1749, 1265; HRMS calcd for $\mathrm{C}_{27} \mathrm{H}_{22} \mathrm{O}_{4} 410.1518$, found 410.1526 .

\section{Diethyl 3-[(E)-3-ethoxy-3-oxoprop-1-enyl]-2-hexyl-1H-indene-1,1-dicarboxylate}

(89). The indicated compound was obtained as a pale yellow oil in a $36 \%$ yield: ${ }^{1} \mathrm{H}$ NMR $\left(\mathrm{CDCl}_{3}\right) \delta 0.89(\mathrm{t}, J=6.9 \mathrm{~Hz}, 3 \mathrm{H}), 1.22-1.49(\mathrm{~m}, 17 \mathrm{H}), 2.73(\mathrm{~m}, 2 \mathrm{H})$, 4.15-4.33 (m, 6H), 6.55 (d, $J=16.5 \mathrm{~Hz}, 1 \mathrm{H}), 7.27$ (dt, $J=0.9,7.5 \mathrm{~Hz}, 1 \mathrm{H}), 7.37$ (dt, $J$ $=0.9,7.5 \mathrm{~Hz}, 1 \mathrm{H}), 7.56$ (d, $J=7.5 \mathrm{~Hz}, 1 \mathrm{H}), 7.62$ (d, $J=7.2 \mathrm{~Hz}, 1 \mathrm{H}), 7.77$ (d, $J=16.5$ $\mathrm{Hz}, 1 \mathrm{H}) ;{ }^{13} \mathrm{C} \mathrm{NMR}\left(\mathrm{CDCl}_{3}\right) \delta 14.2,14.3,14.5,16.6,22.8,28.4,30.1,30.5,31.7,60.9$, 62.4, 72.0, 120.7, 121.5, 125.4, 126.4, 128.9, 136.2, 136.9, 140.2, 142.7, 151.2, 167.3, 168.1; IR $\left(\mathrm{CHCl}_{3}, \mathrm{~cm}^{-1}\right)$ 3054, 2985, 1727, 1718, 1265; HRMS calcd for $\mathrm{C}_{26} \mathrm{H}_{34} \mathrm{O}_{6}$ 442.2355, found 442.2362. 


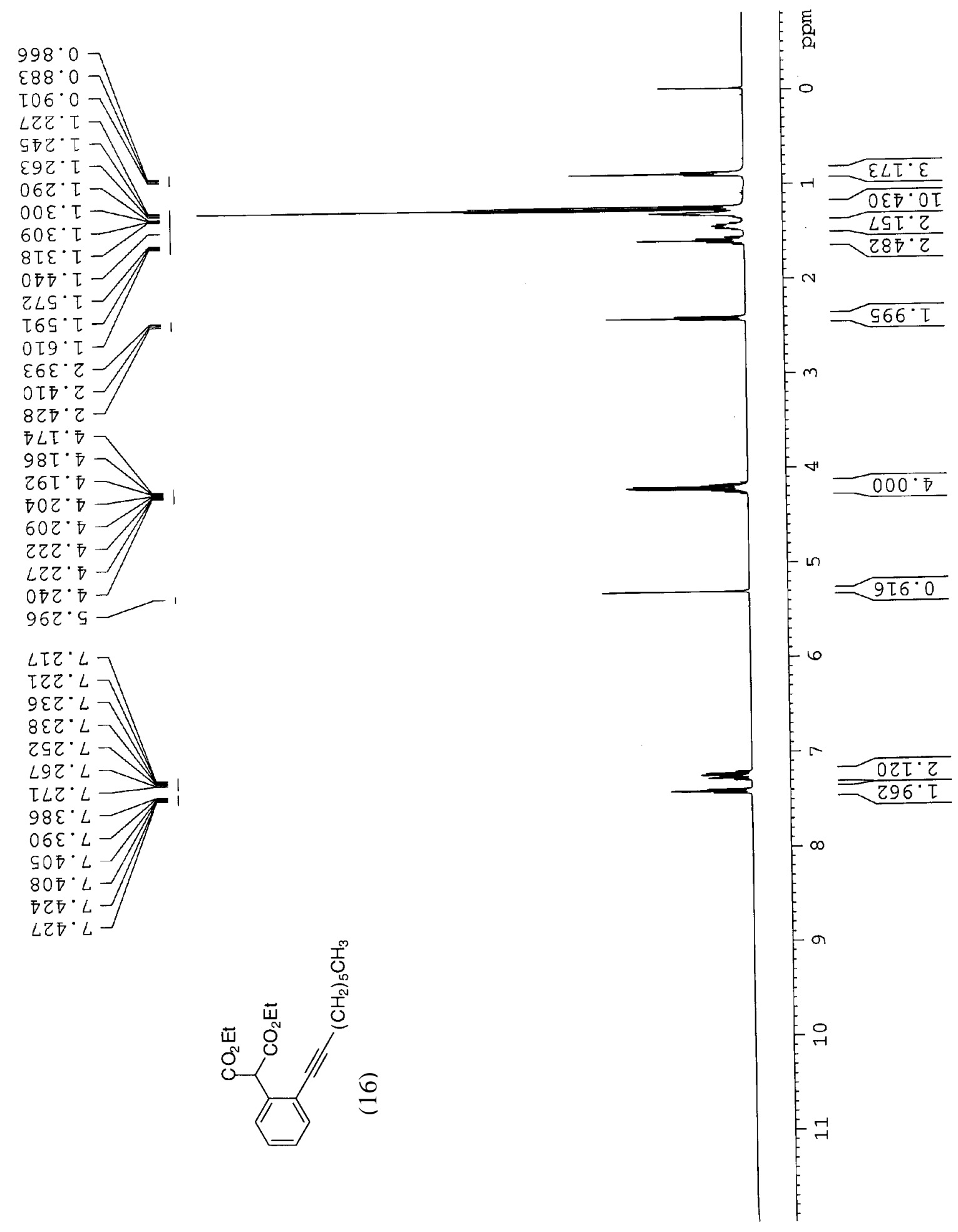



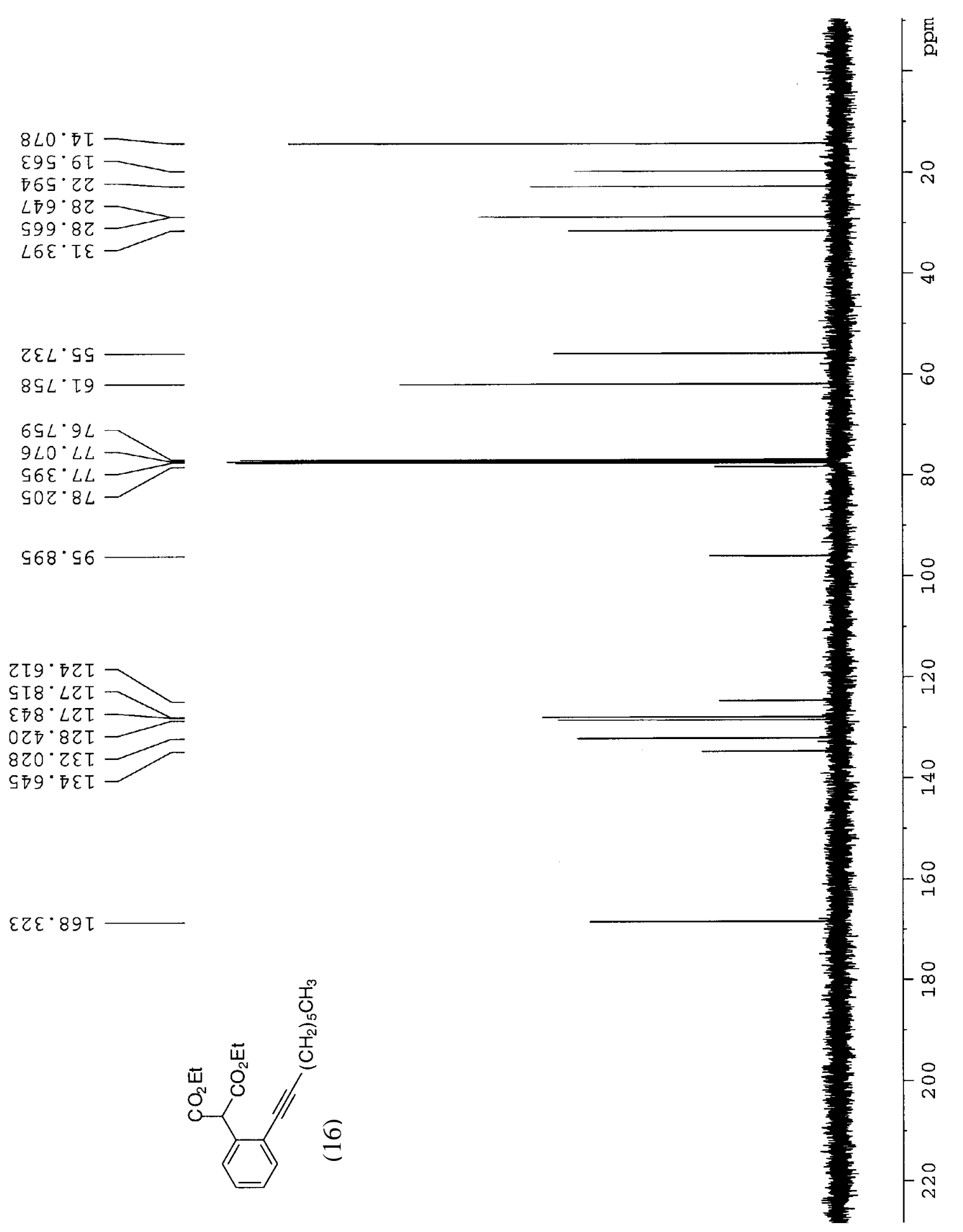

$\varepsilon Z \varepsilon^{\circ} 89 \tau$

$9 \angle 0 \cdot \angle L$

$96 \varepsilon^{\circ} L L$

$S 0 Z \cdot 8 L$

$\varsigma 68 \cdot 96$

$8 Z 0^{\circ} Z \varepsilon \mathrm{L}$ 


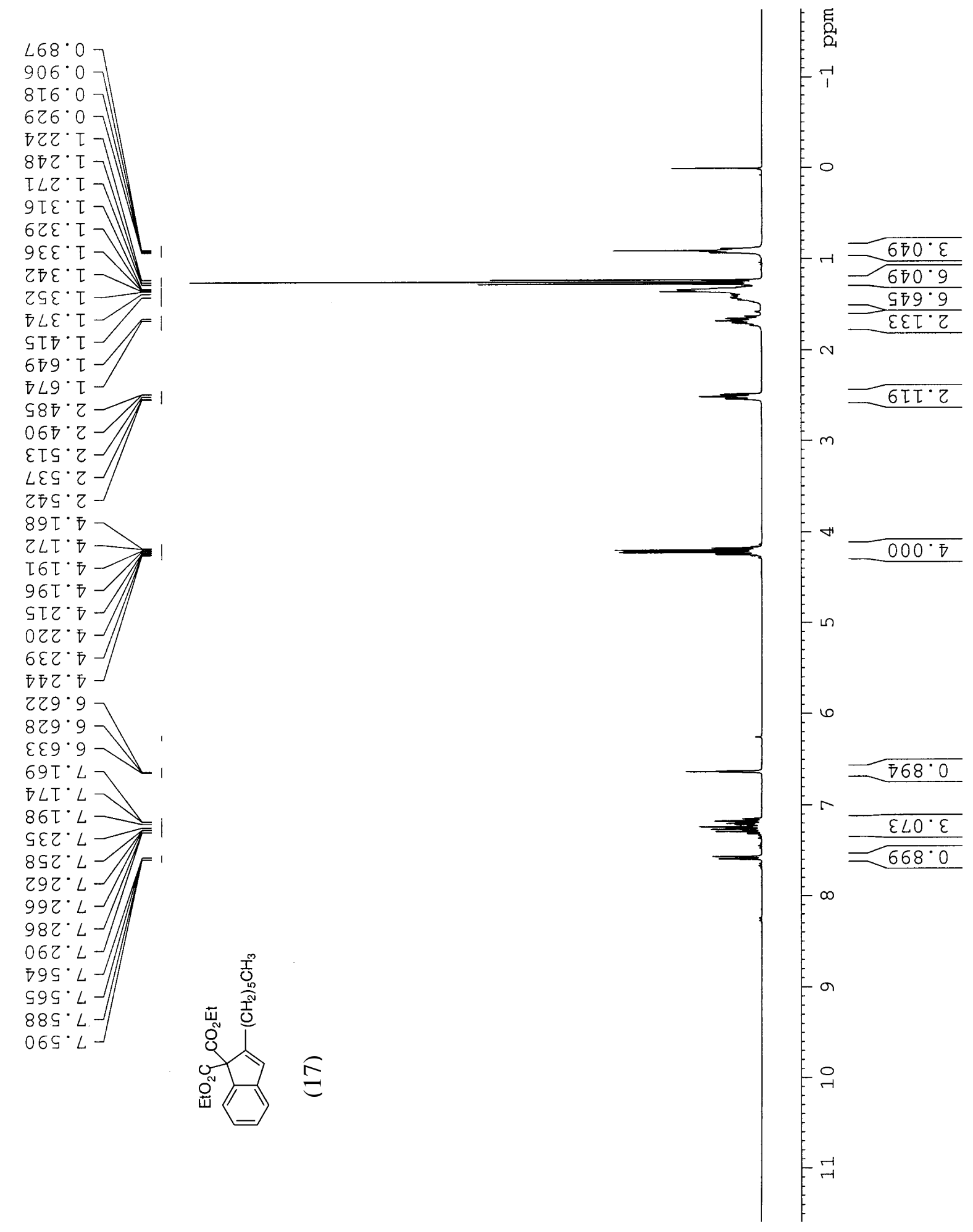




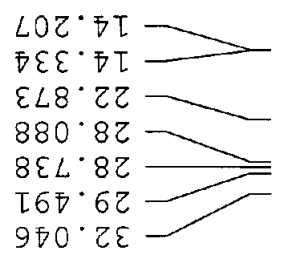

$660 \cdot 29$

ZT2・ $2 L$

$678 \cdot 9 L$

$Z L C \cdot L L-$
$969 \cdot L L$

8SL.0ZI

$8 \tau 0^{\circ} \mathrm{gzT} \longrightarrow$

$\varepsilon 乙 \downarrow \cdot \varsigma \longrightarrow$

$99 L^{\circ} 8 z \tau=$
$9 \varepsilon 8 \cdot 6 z \tau=-$

$\angle \angle 8^{\circ}$ ODI

$889^{\circ}$ 元

$975^{\circ} 87$

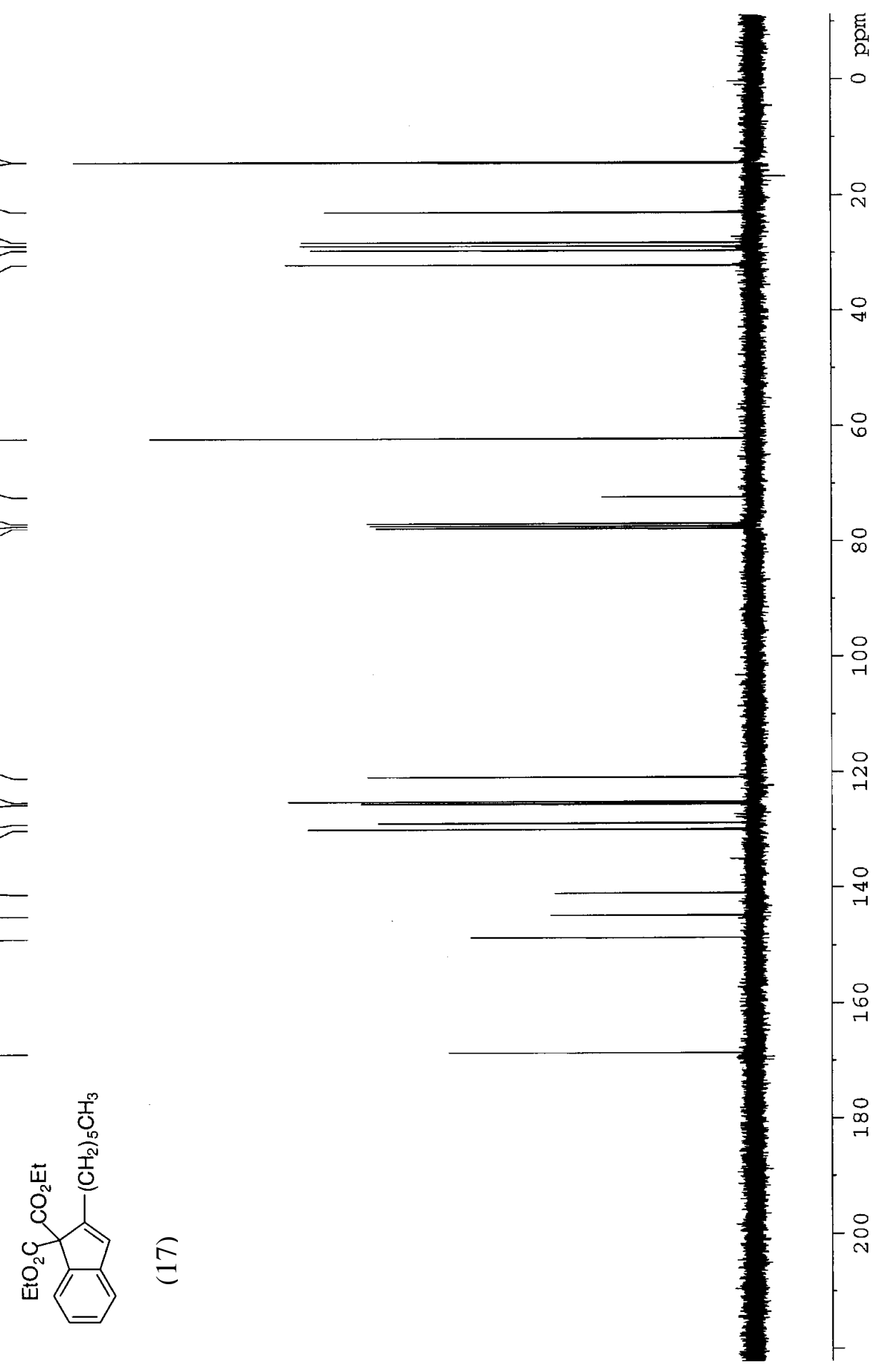

$2 T 5 \cdot 89 T$

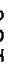

总

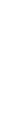




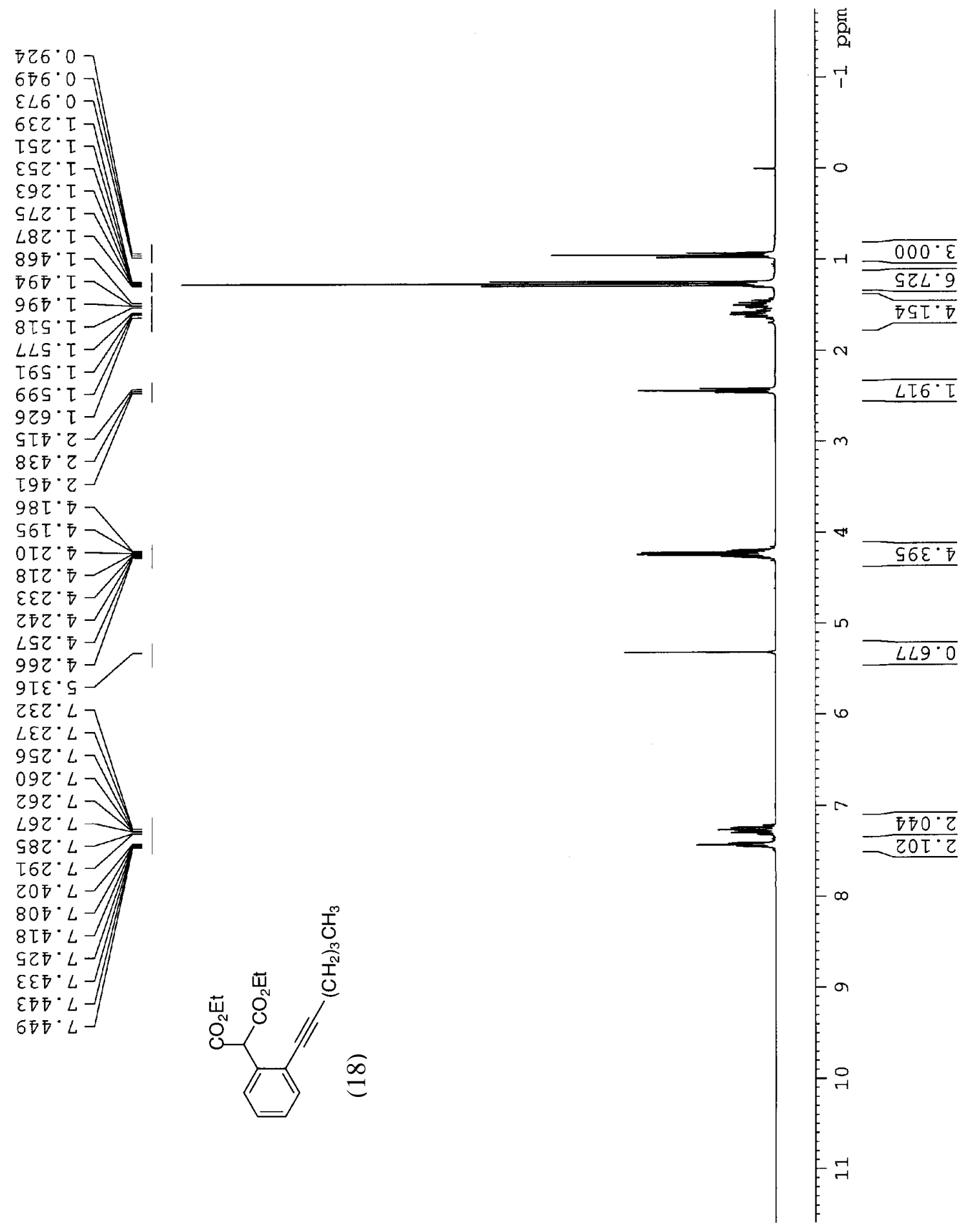


$299^{\circ} \varepsilon \tau \longrightarrow$

$\angle \nabla Z \cdot 6 I$

$620^{\circ} \mathrm{ZZ}$

$8 \nabla L \cdot 0 \varepsilon$

$6 \varepsilon L \cdot S 9$

$\varepsilon L L^{*}$ โ9

$008 \cdot 9 L$

8II. $L L$

$\nabla 0 乙 \cdot 8 L$

Ђร8・ $ธ 6$

Z65

0 $88^{\circ} \angle Z T$

$298^{\circ} \angle Z T$

$90 \overline{0}^{\circ} 8 Z \mathrm{~L}$

$6 Z 0^{\circ} \mathrm{ZL \tau}$

โ૬9

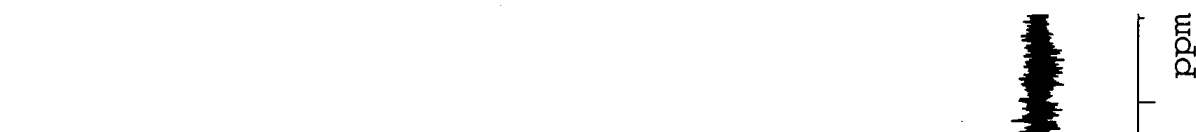

है
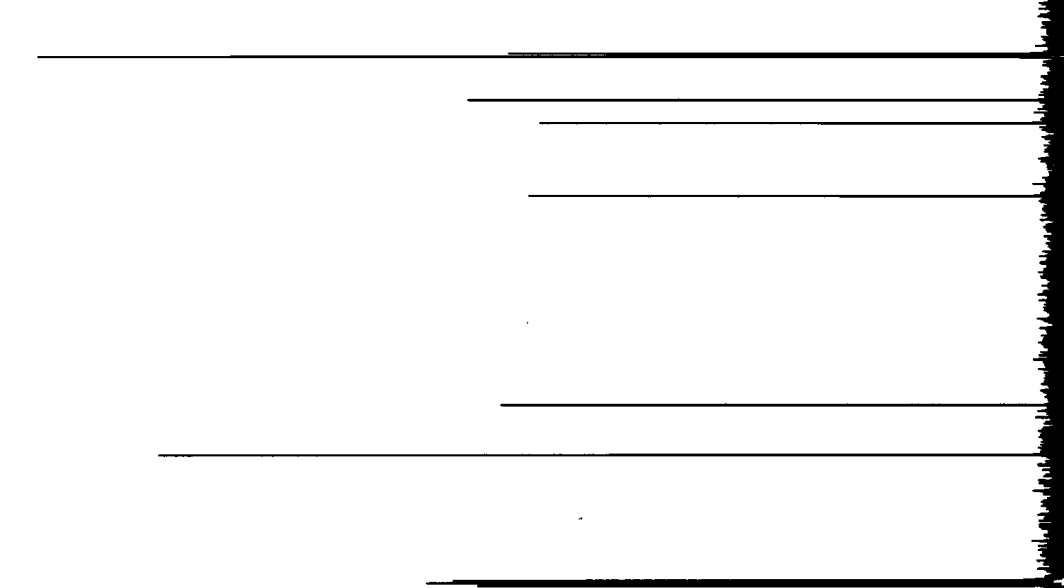


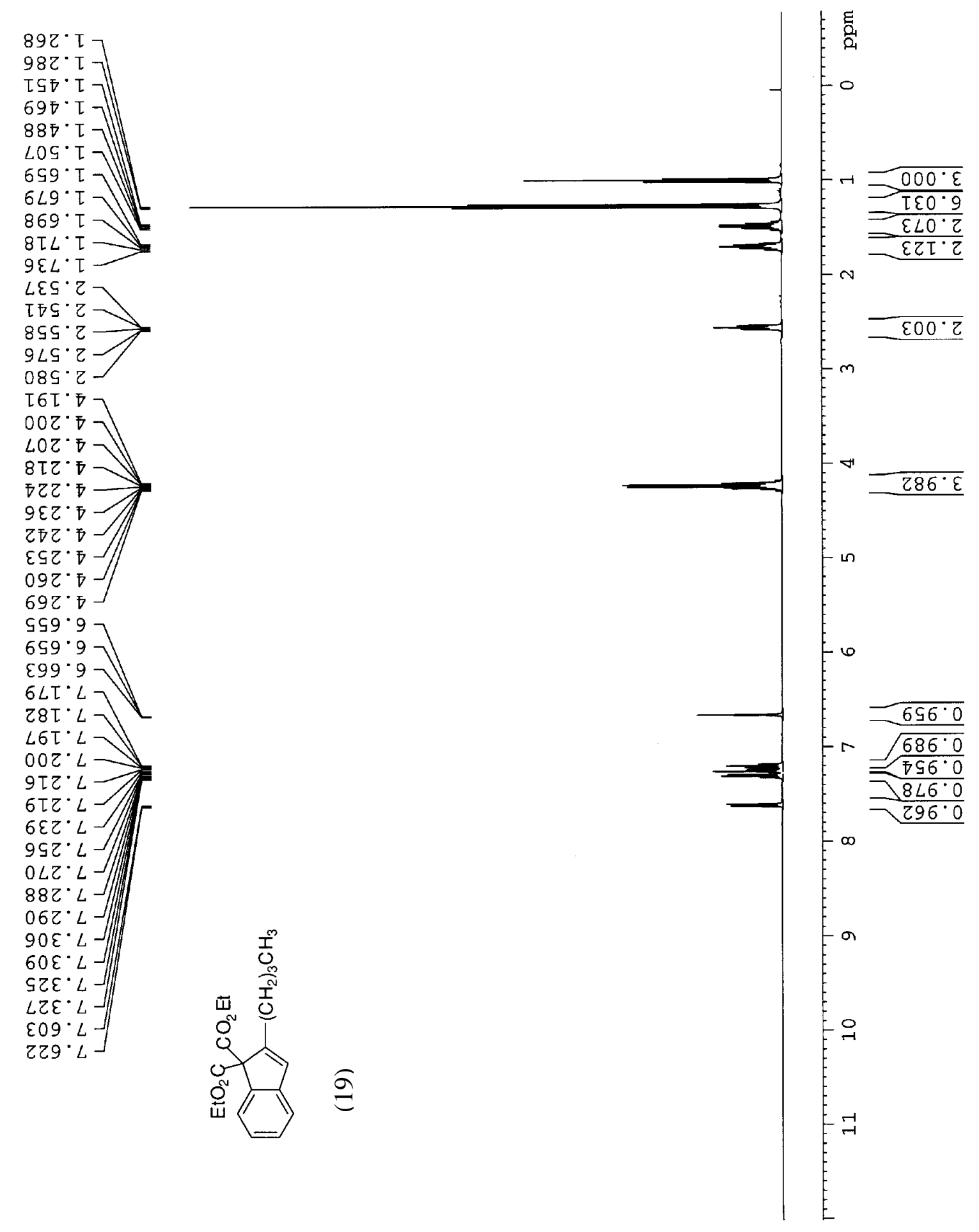


ยEO $0^{\circ} \tau$

$9 \tau \tau \cdot \nabla \tau$

乙โL て乙

$852 \cdot 87$

$860^{\circ} 0 \varepsilon$

จ26 โ9

万๐० $2 L$

$998^{\circ} 9 L$

$78 T^{\circ} L L$

EOS $L L$

$209^{\circ}$ OZI

8モ8. ठ乙L

$\varepsilon 9 \mathrm{C}^{\circ} \mathrm{LLI}$

$\angle 09^{\circ} 8 Z \mathrm{~T}$

$\tau 69^{\circ} 62 \tau$

ITL.ODI

हIS ${ }^{\circ} \bar{\nabla} I$

$\varsigma 8 \mathrm{r} \cdot 8 \bar{T}$

IE\&・89

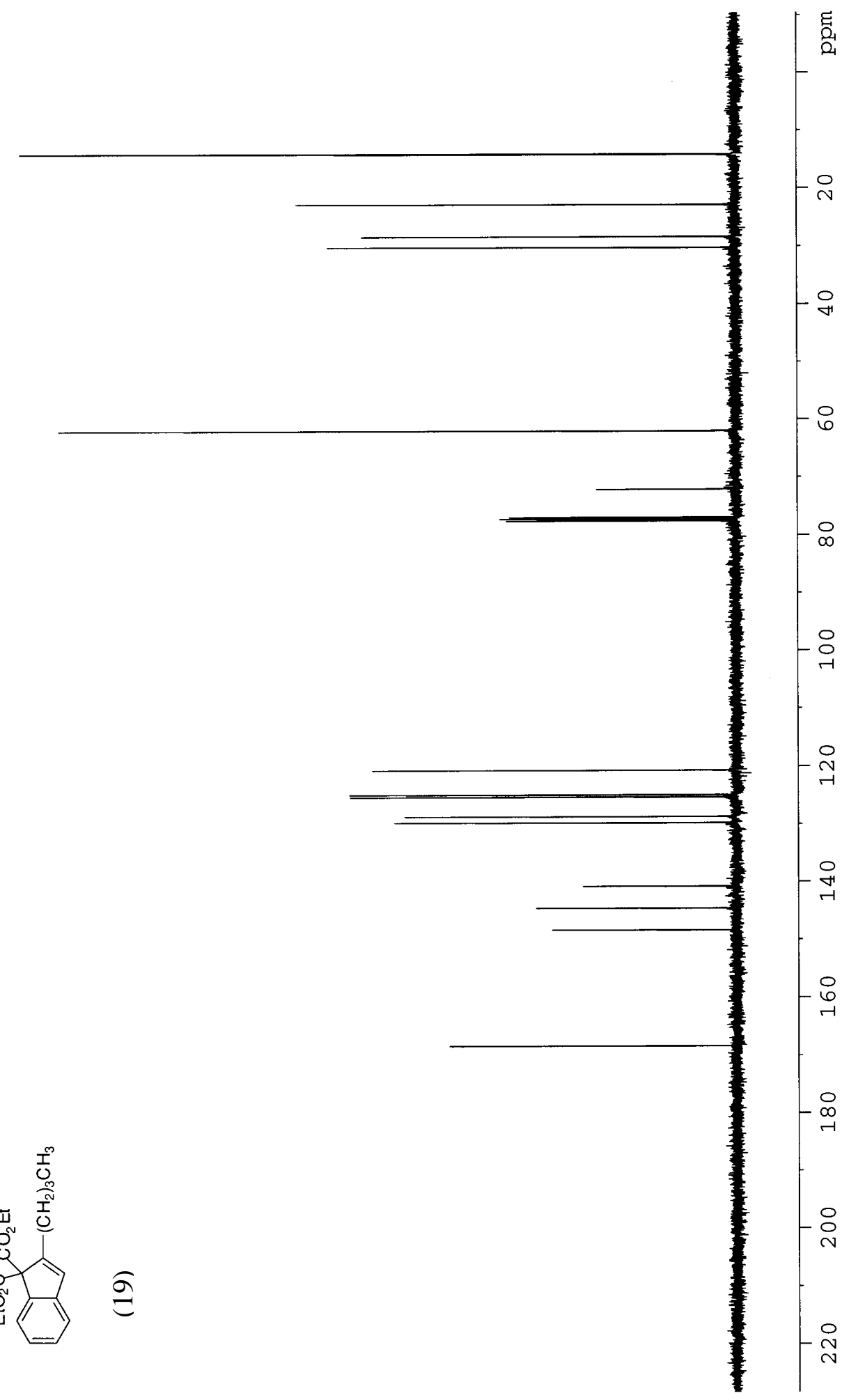




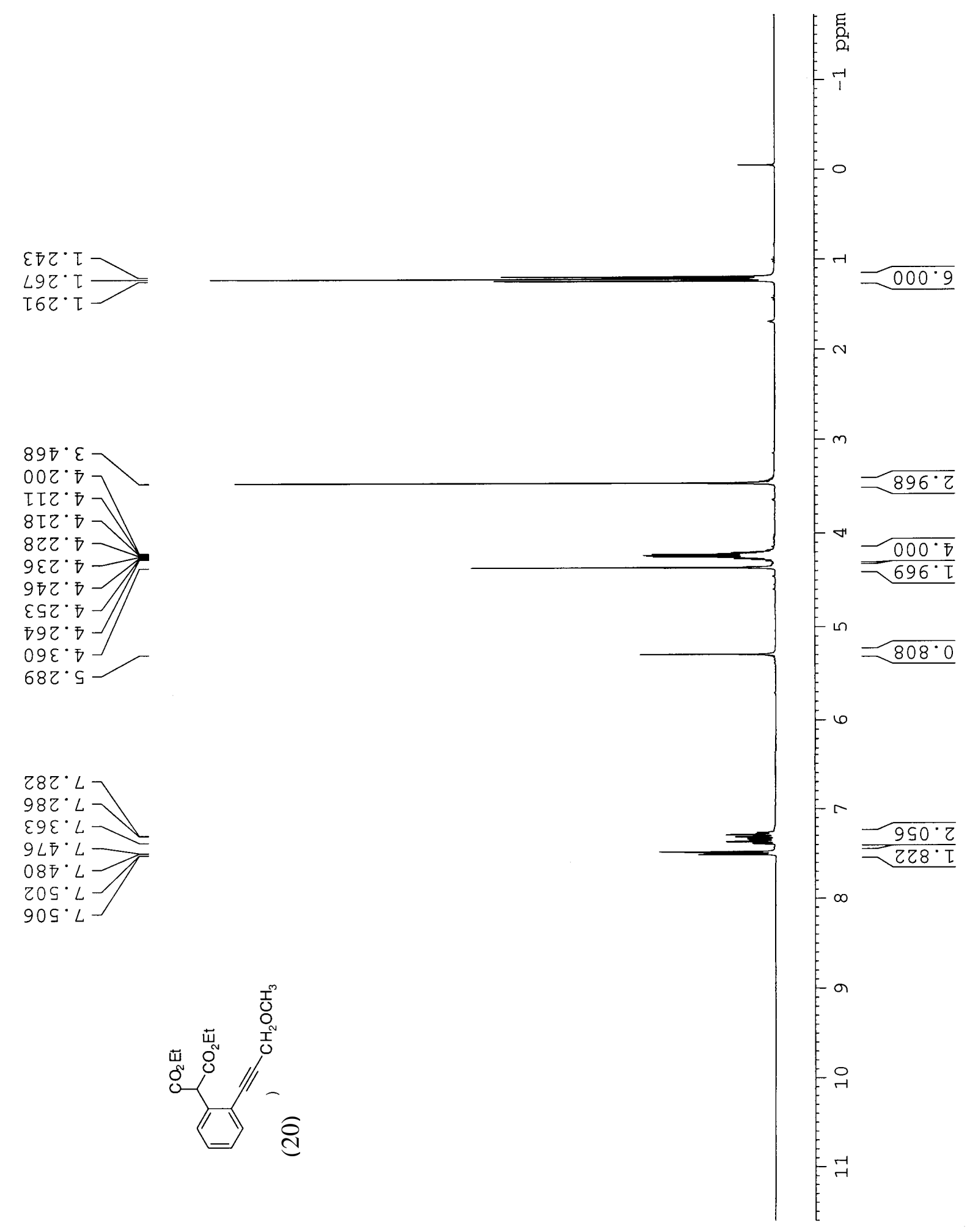


$90 L$ ' 9

$20 \angle . \angle S$

Z6ह. 09

โ26. โ9

ZLL. $9 L$

$060^{\circ} \mathrm{LL}$

$800^{\circ} \angle L$

$096^{\circ} \varepsilon 8$

$680^{\circ} 06$

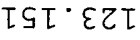

$286^{\circ} \mathrm{LZT}$

ЂعL $8 Z \tau$

$888^{\circ} 8 Z \mathrm{~T}$

$0 \angle \sigma^{\circ} Z \varepsilon \tau$

$\varepsilon 06^{\circ} \nabla \varepsilon T$

५०I·89

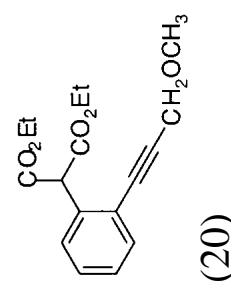

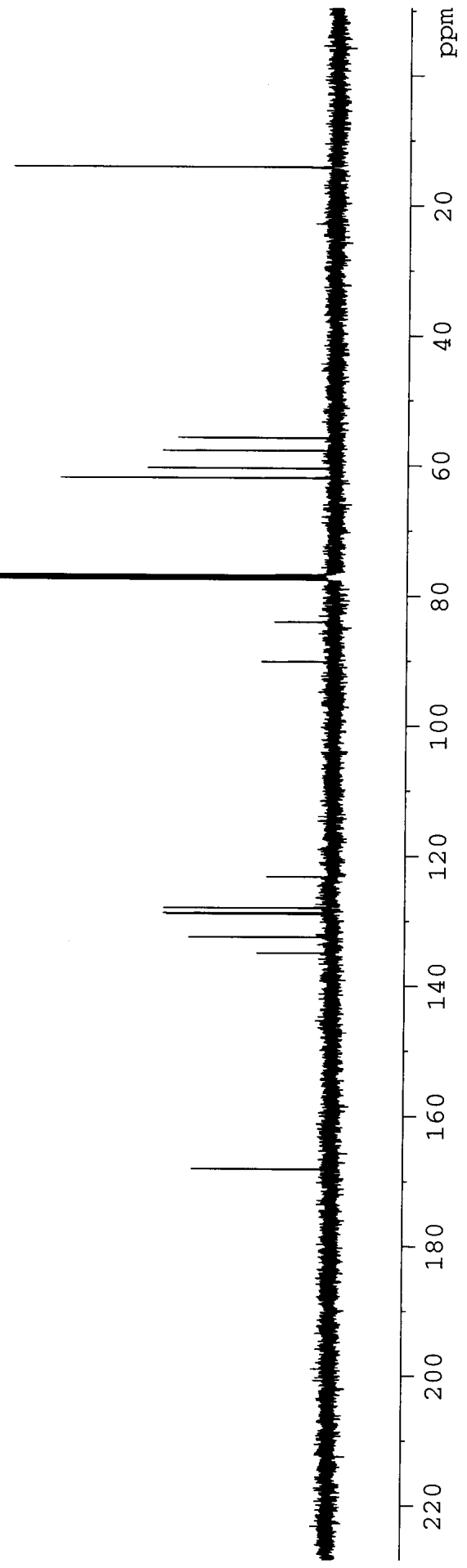




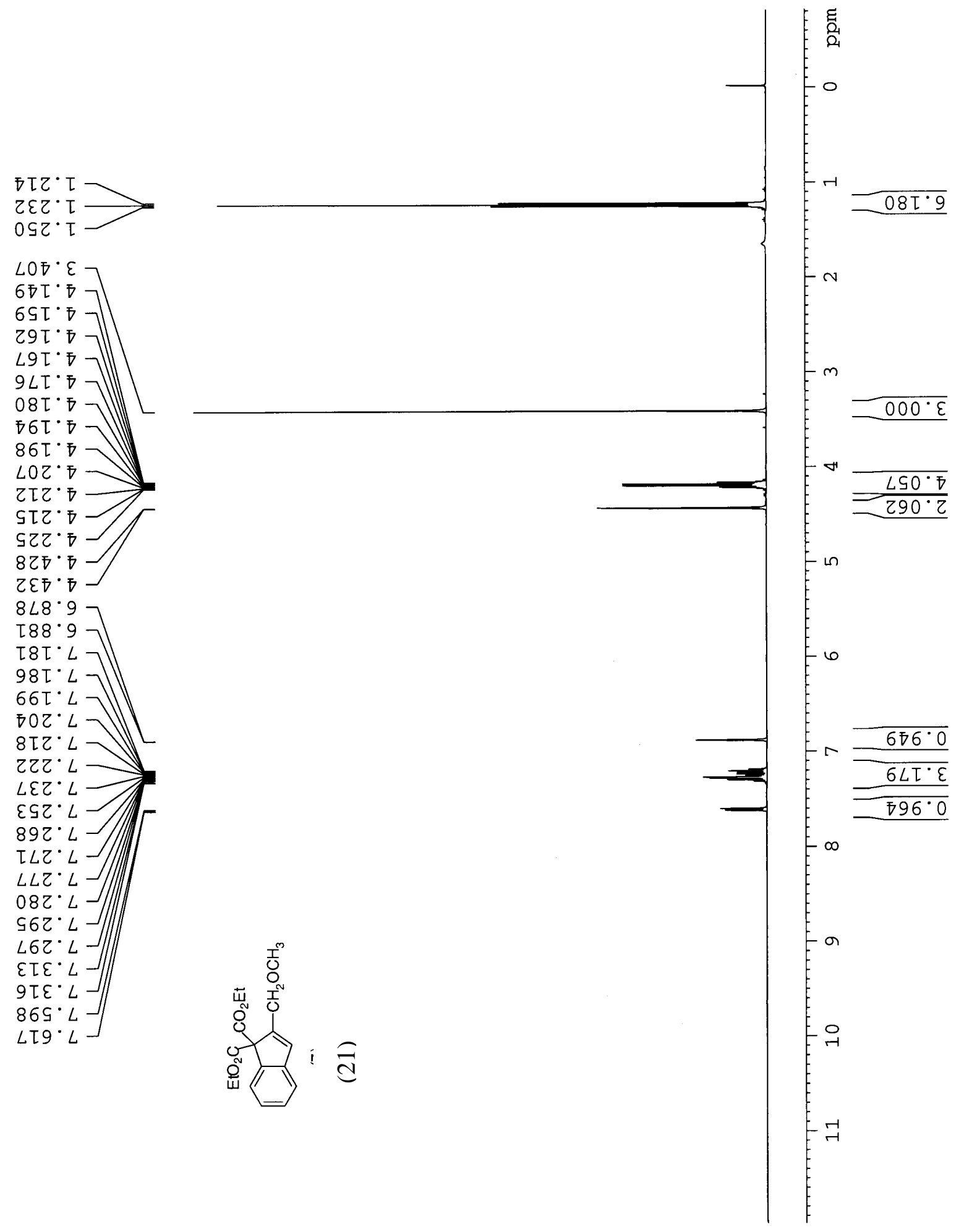




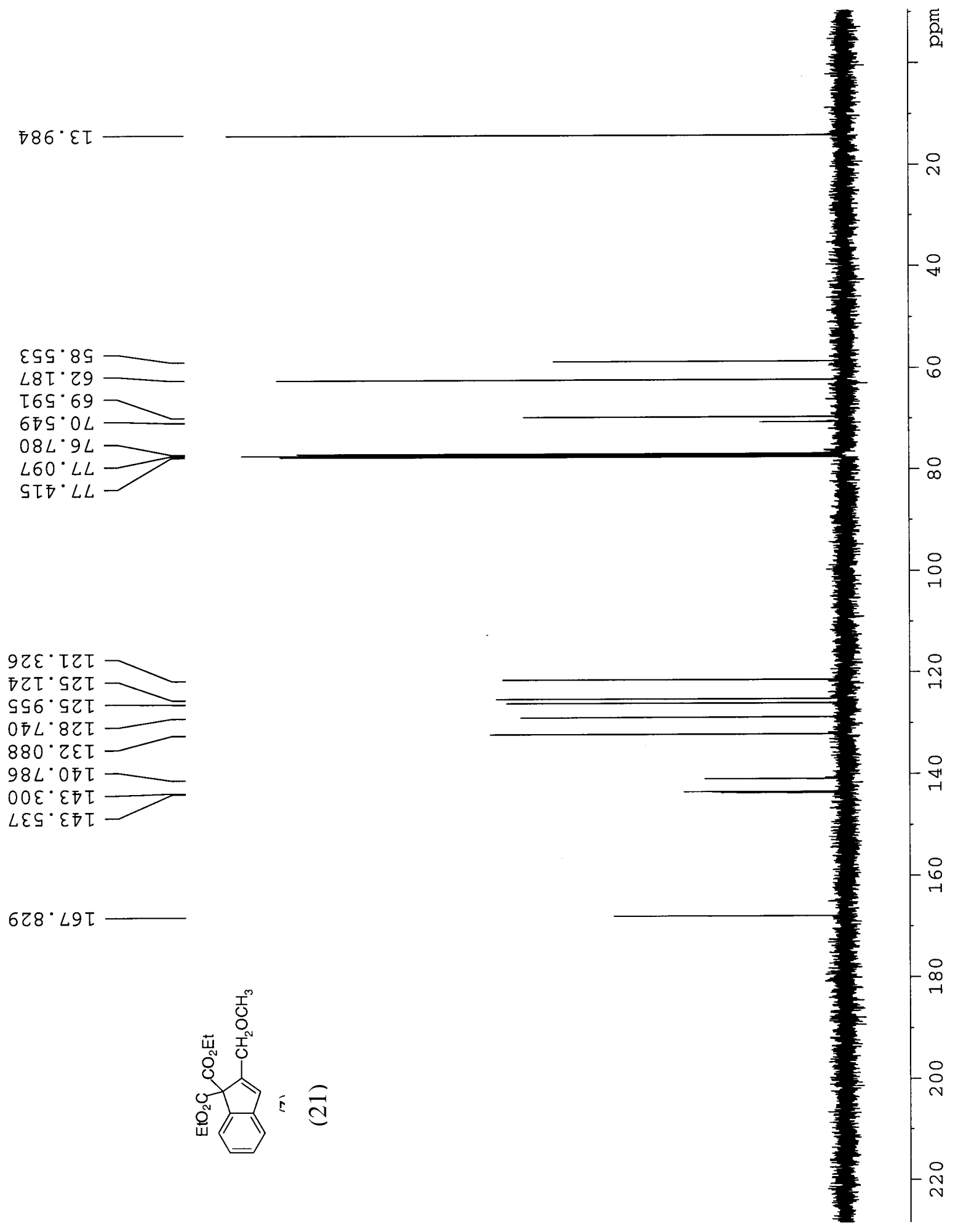




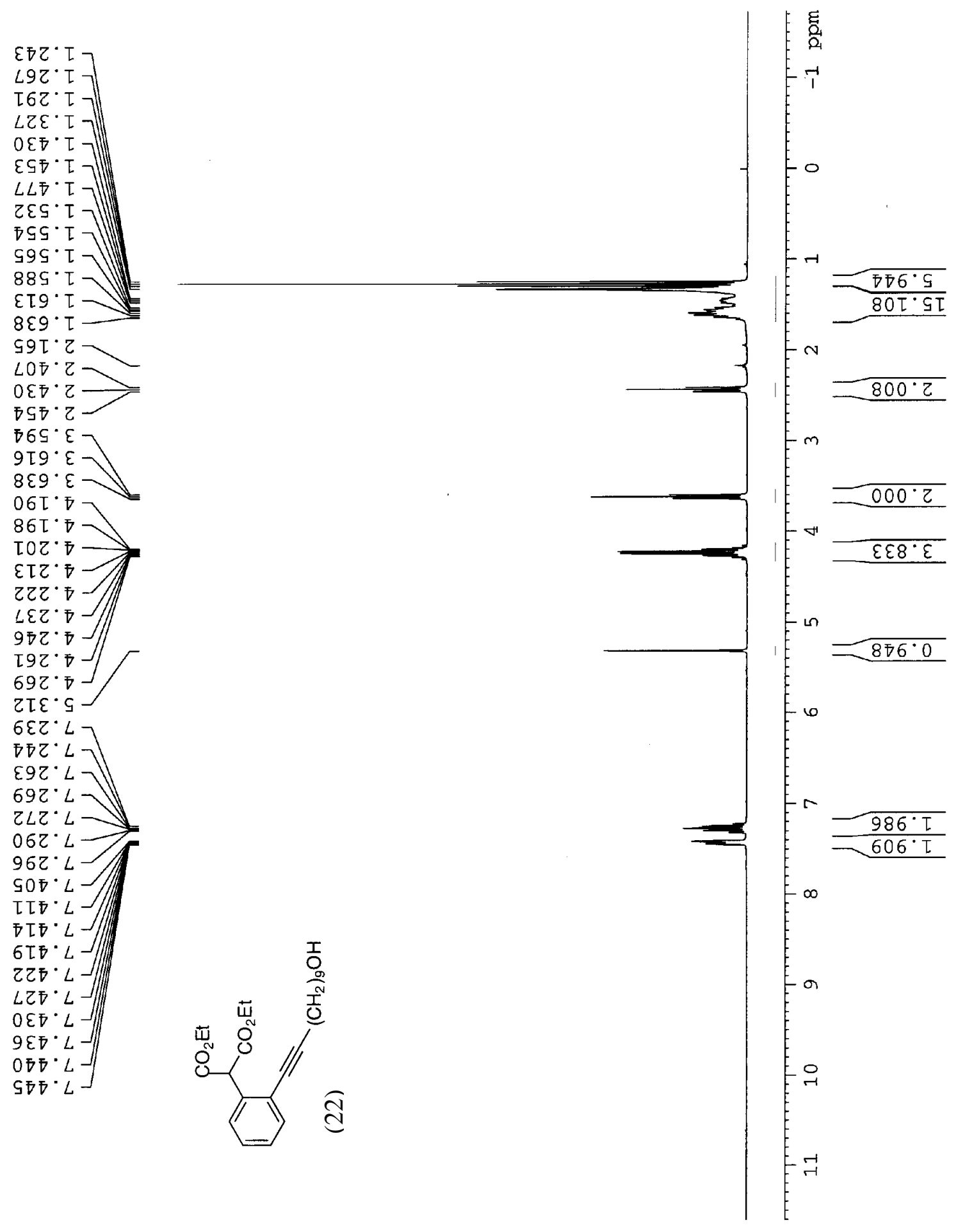


$892^{\circ}$

万乙L・6

$076^{\circ} \mathrm{sz}$

ர $8^{\circ} 8 \mathrm{Z}$

OT. $\cdot 62$

$782 \cdot 62$

$109^{\circ} 62$

โ $89^{\circ} 62$

L $\angle 6^{\circ} \mathrm{ZE}$

$976^{\circ} 9 \mathcal{G}$

$\angle 96^{\circ}$ โ9

टLI. $19=$

$9 L 8 \cdot 9 L$

$662^{\circ} L L$

$\overline{Z Z L} L L$

$\varepsilon 6 \varepsilon \cdot 8 L$

$290 \cdot 96$

79L. $72 T$

$600.82 \mathrm{~T}$

$170.82 \mathrm{~T}$

$085^{\circ} 82 \mathrm{~T}$

ZTZ ZET

$908^{\circ}$ ธहL

$\varepsilon I S^{\cdot} 89 T$

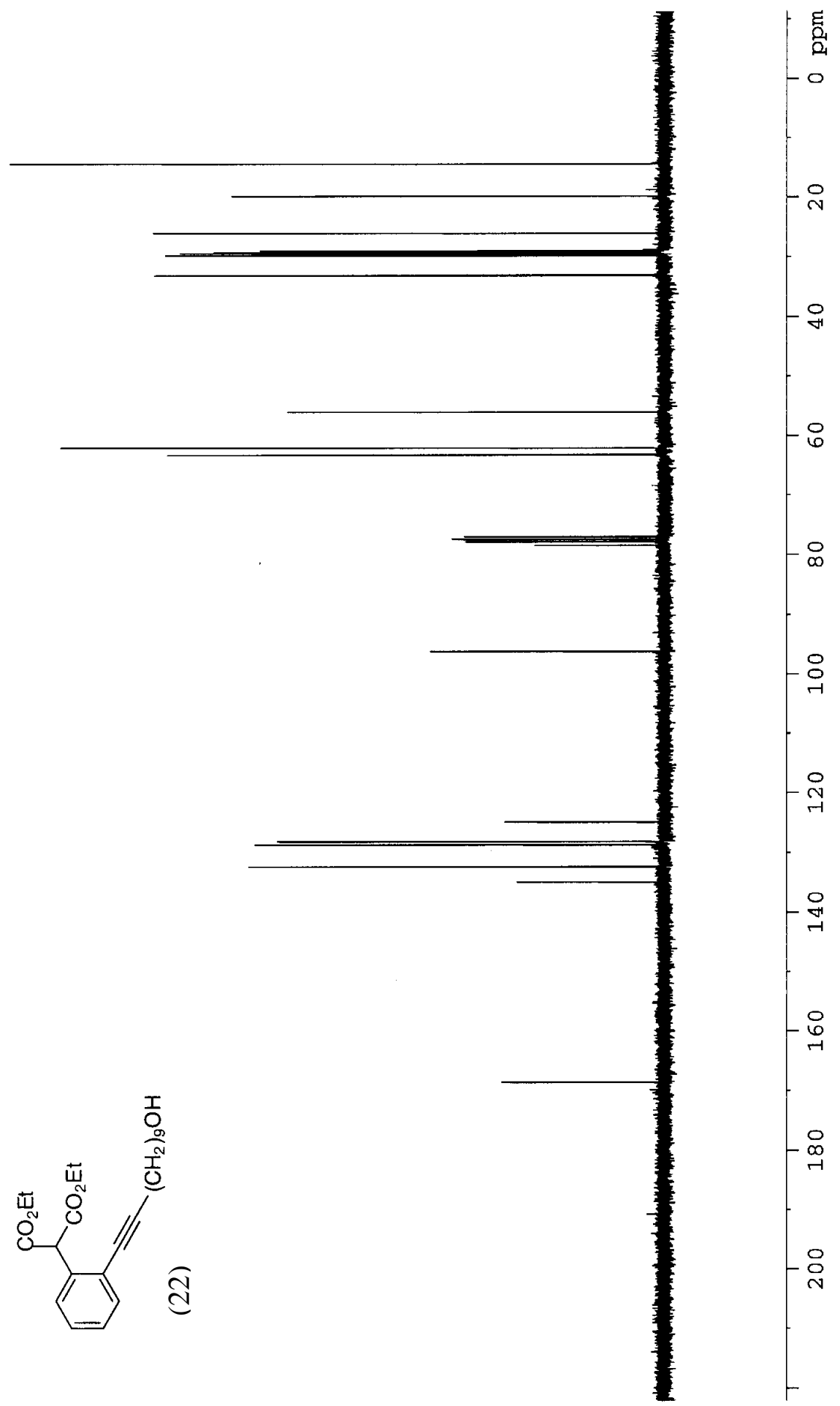




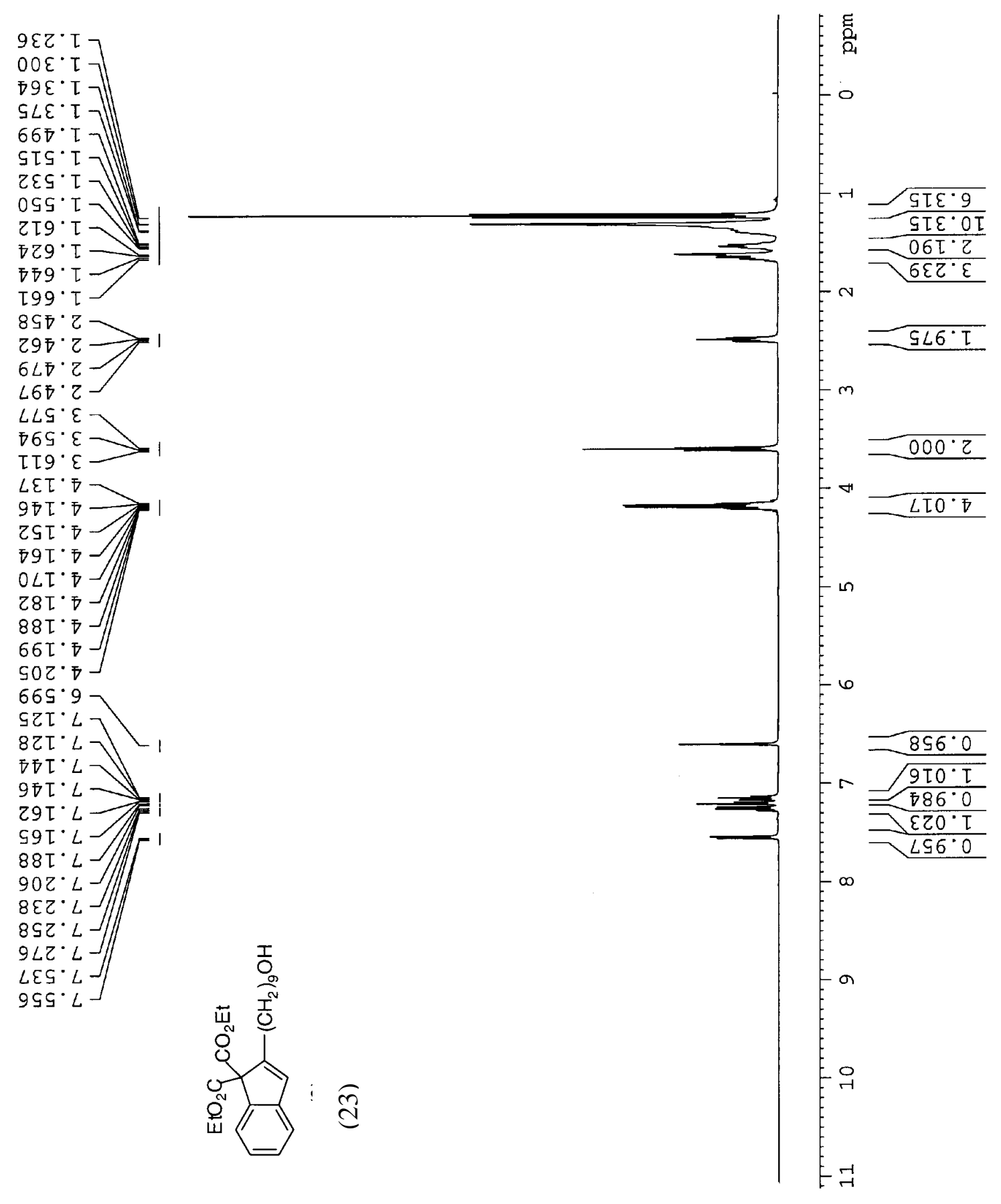



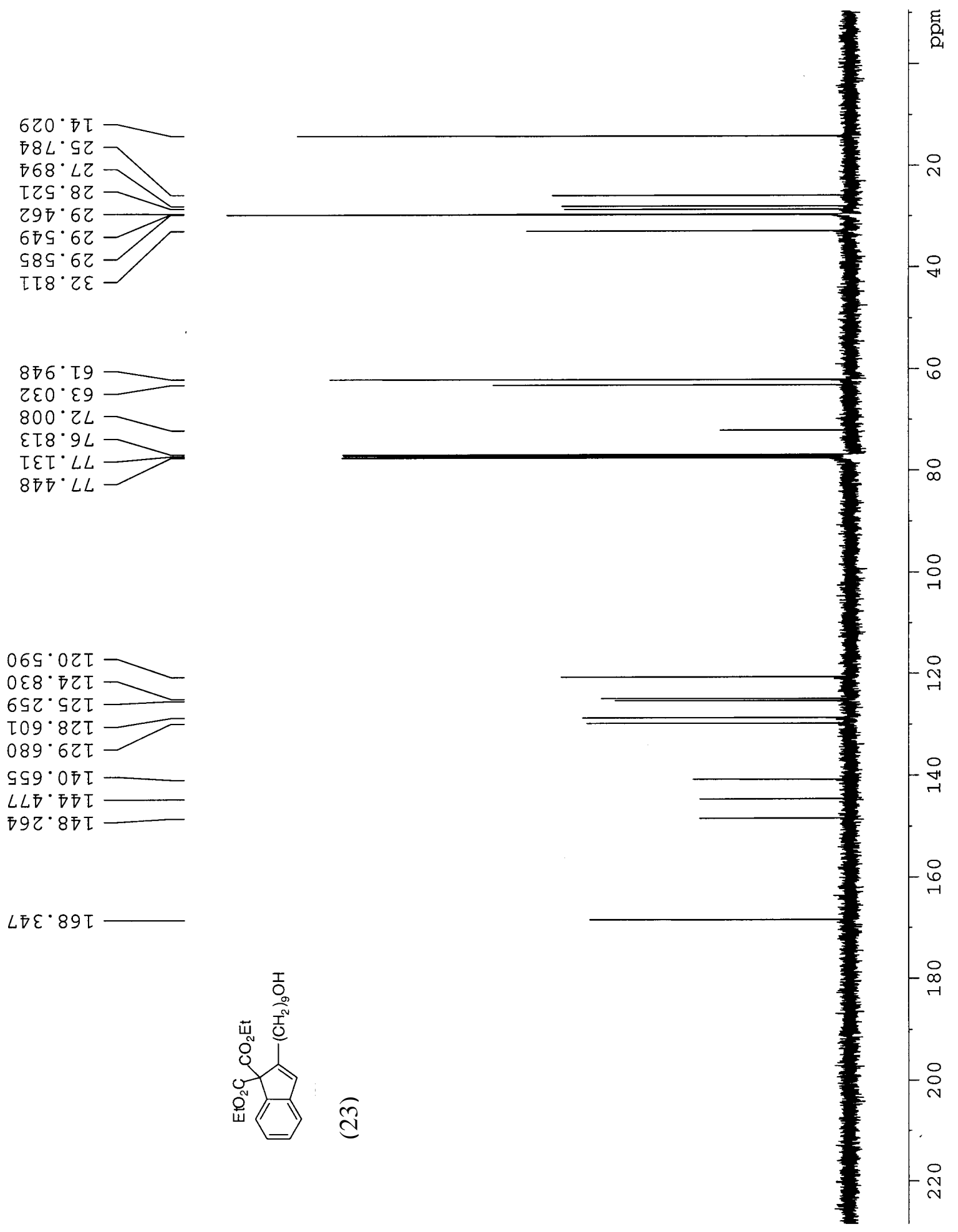


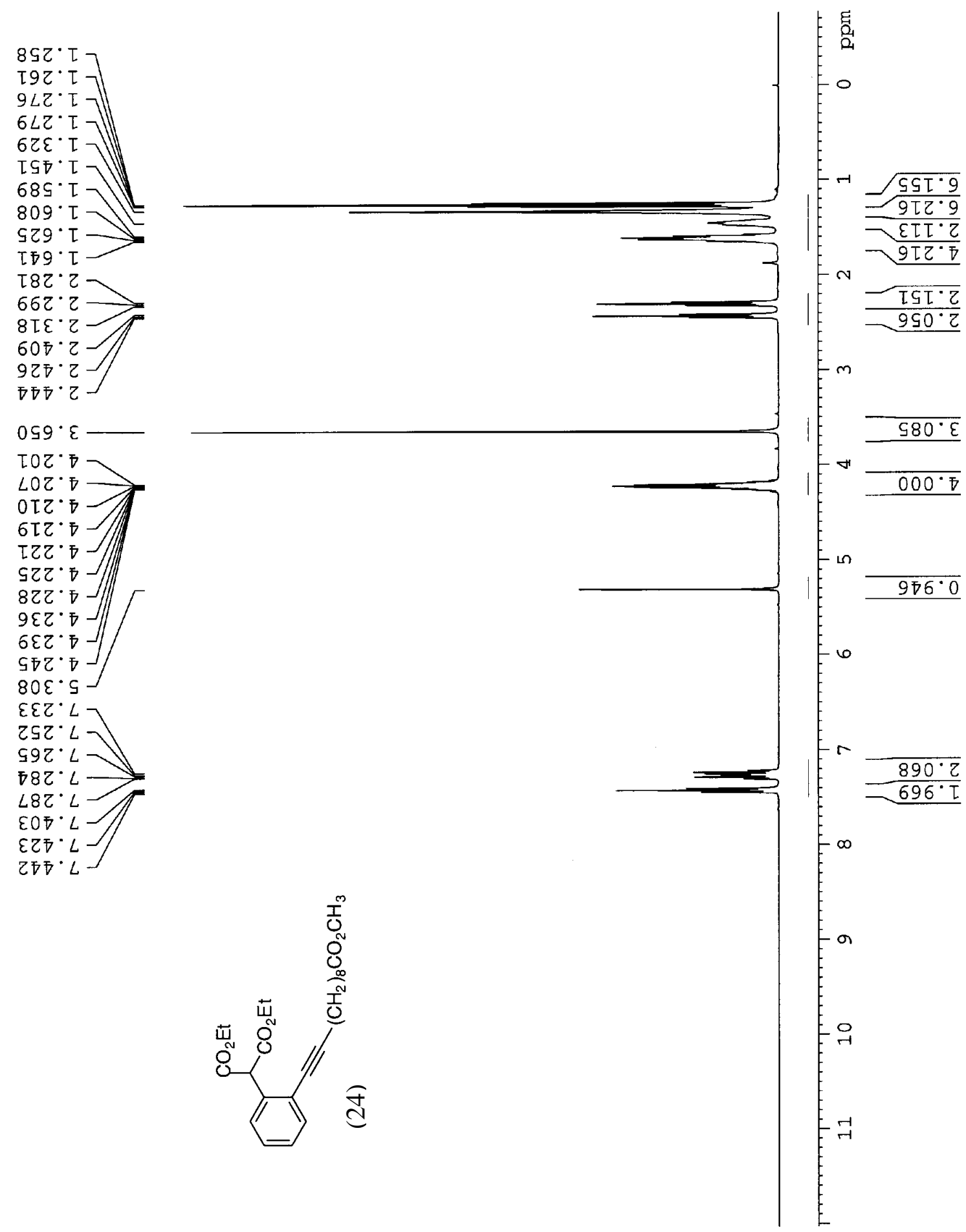




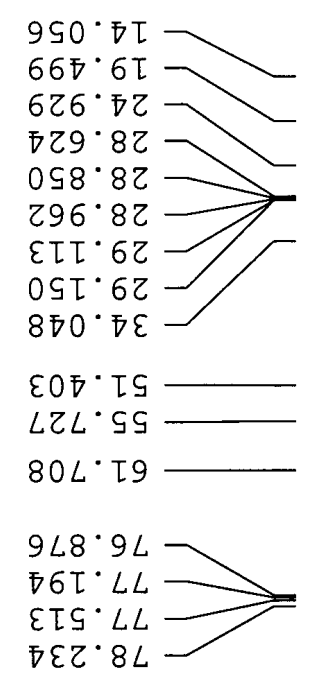

ILL· S 6
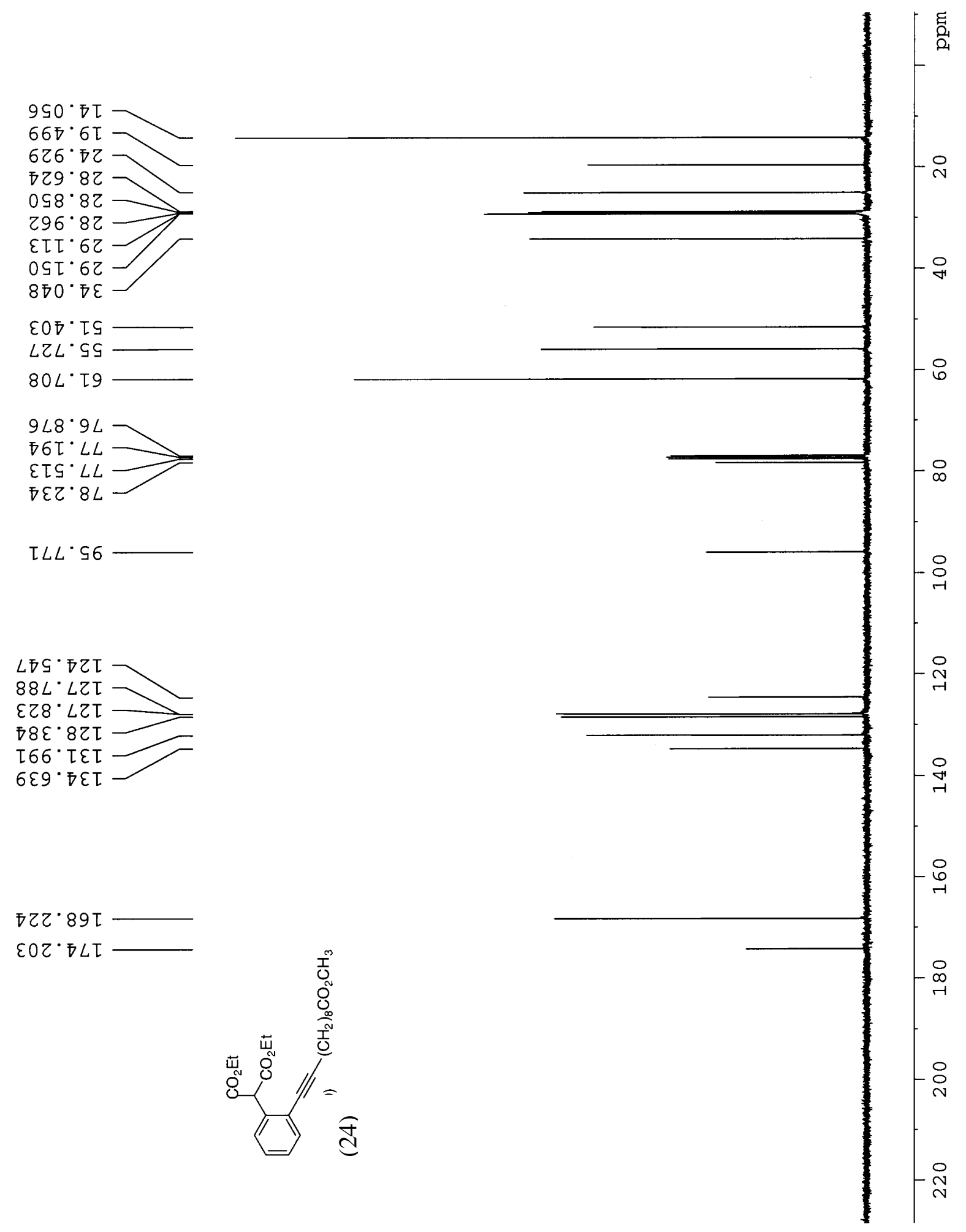


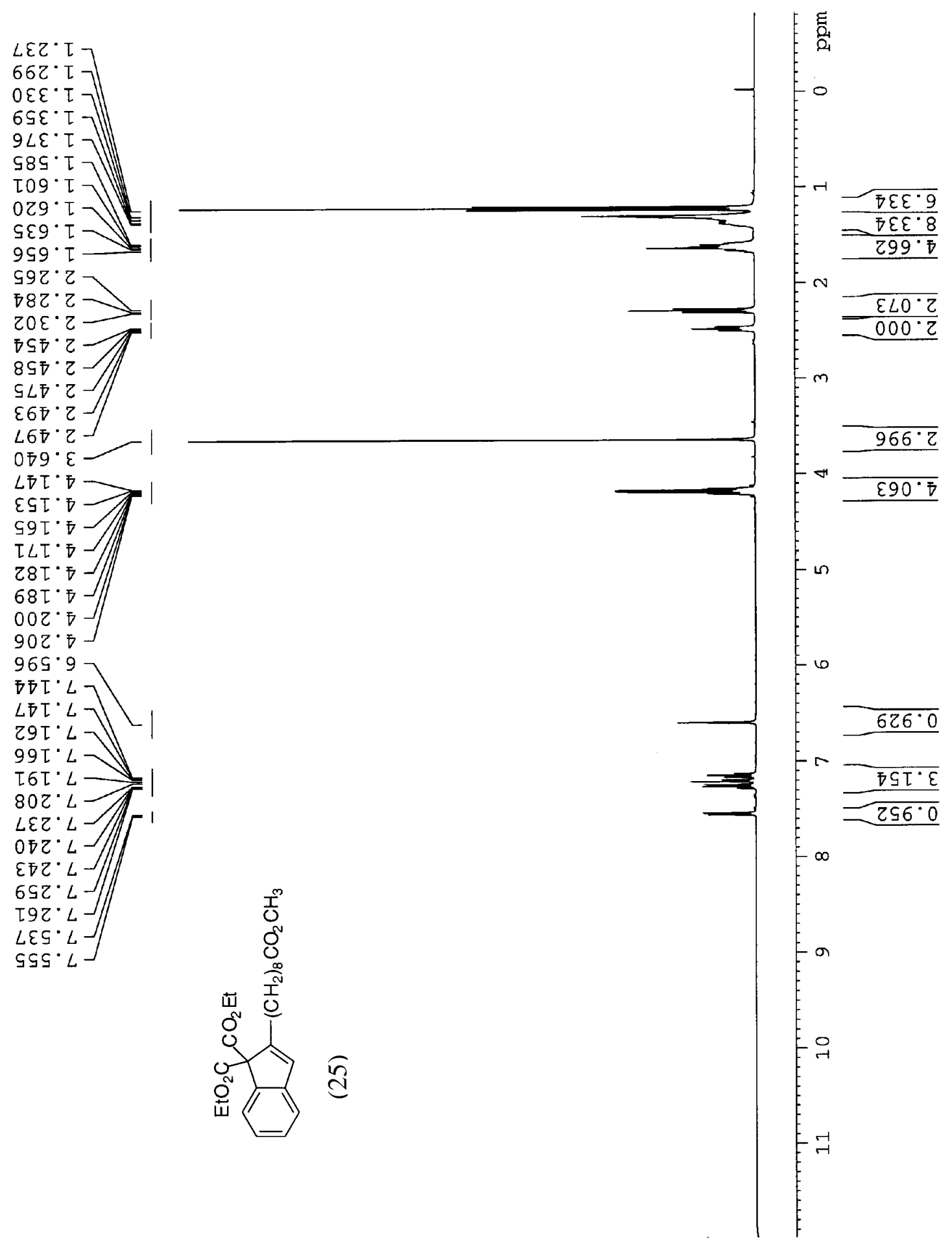



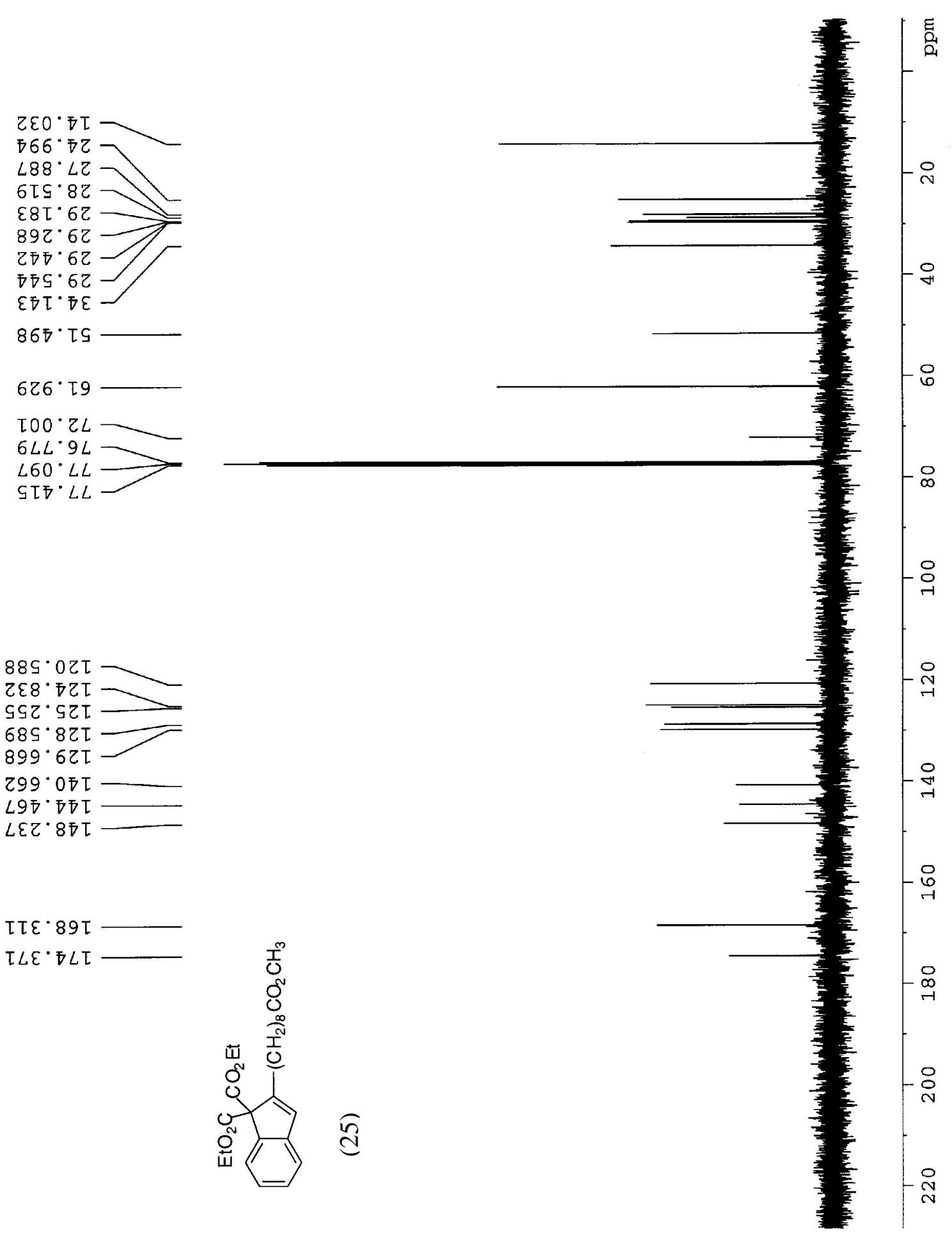

$889^{\circ} 0 \mathrm{0Z} \longrightarrow$

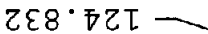

$\mathrm{gSZ} \cdot \mathrm{GZT} \longrightarrow$

$68 \mathrm{~s}^{\circ} 8 \mathrm{zT} \longrightarrow$

$899^{\circ} 62 \mathrm{ZT}$

$299^{\circ} 07 \mathrm{~T}$

$\angle 9 \bar{\circ}^{\circ}$ 元

$\angle \varepsilon Z \cdot 8 T T$

$\tau \tau \varepsilon \cdot 89 \tau$

$\tau L \varepsilon^{\circ} \nabla L \tau$

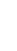

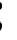




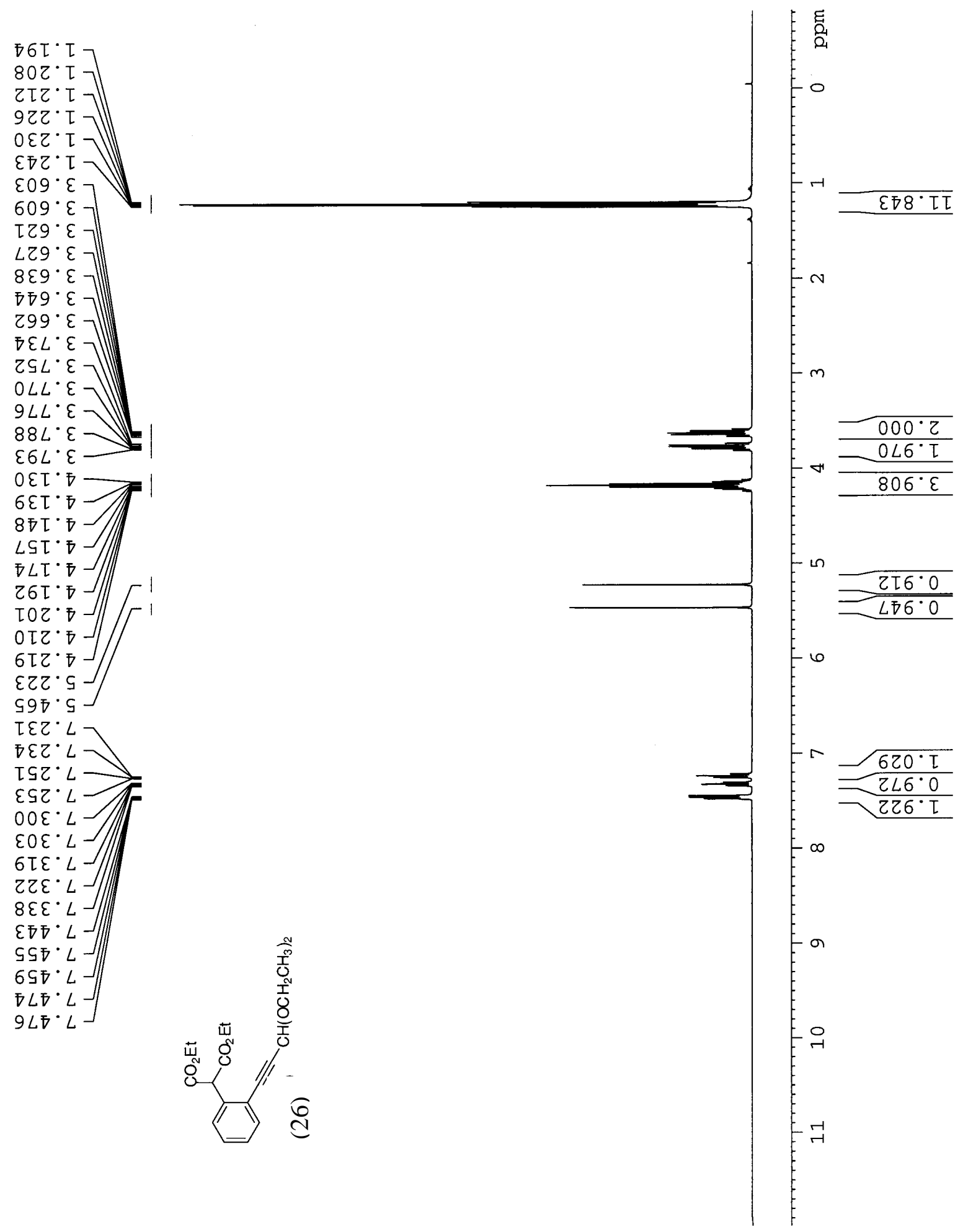




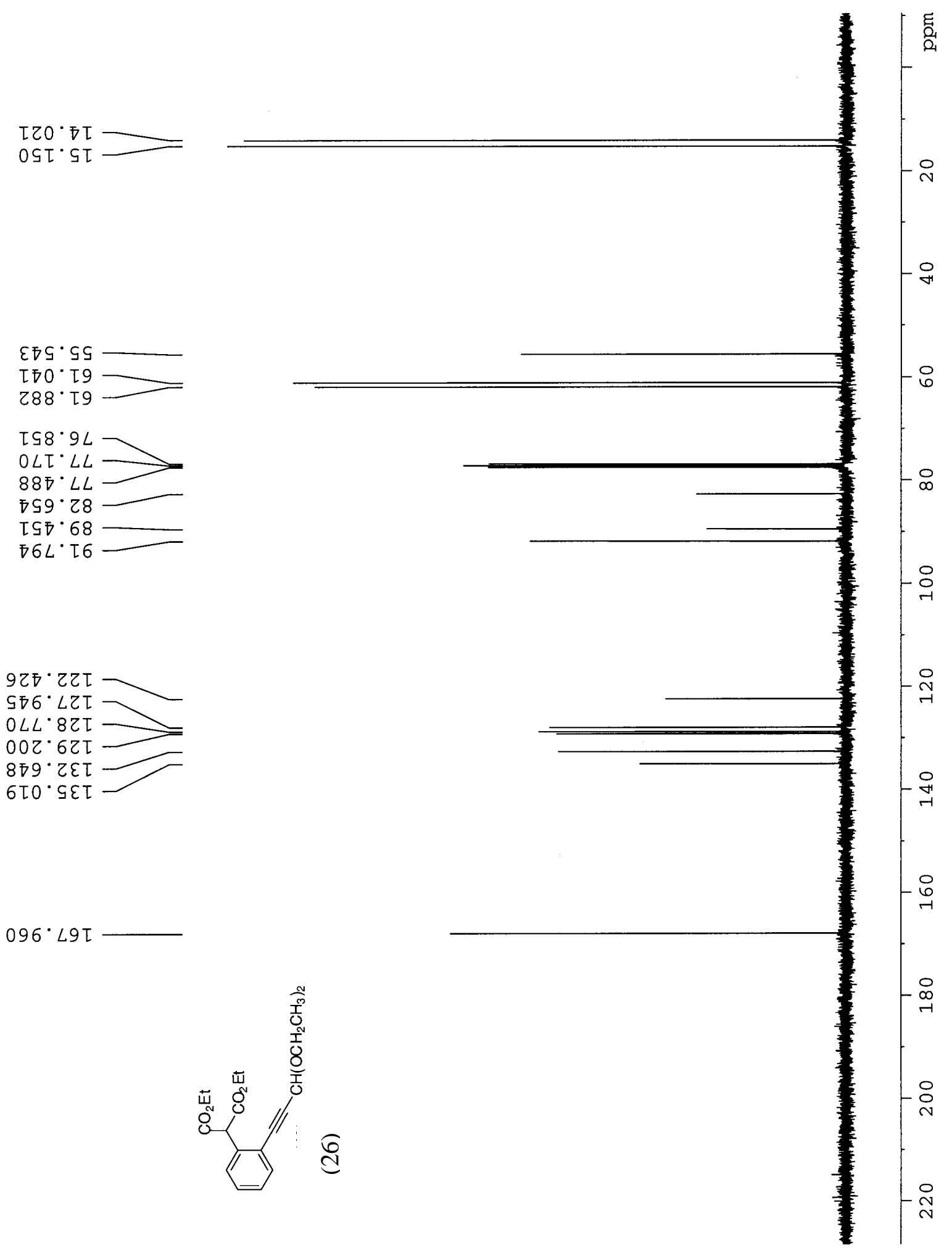




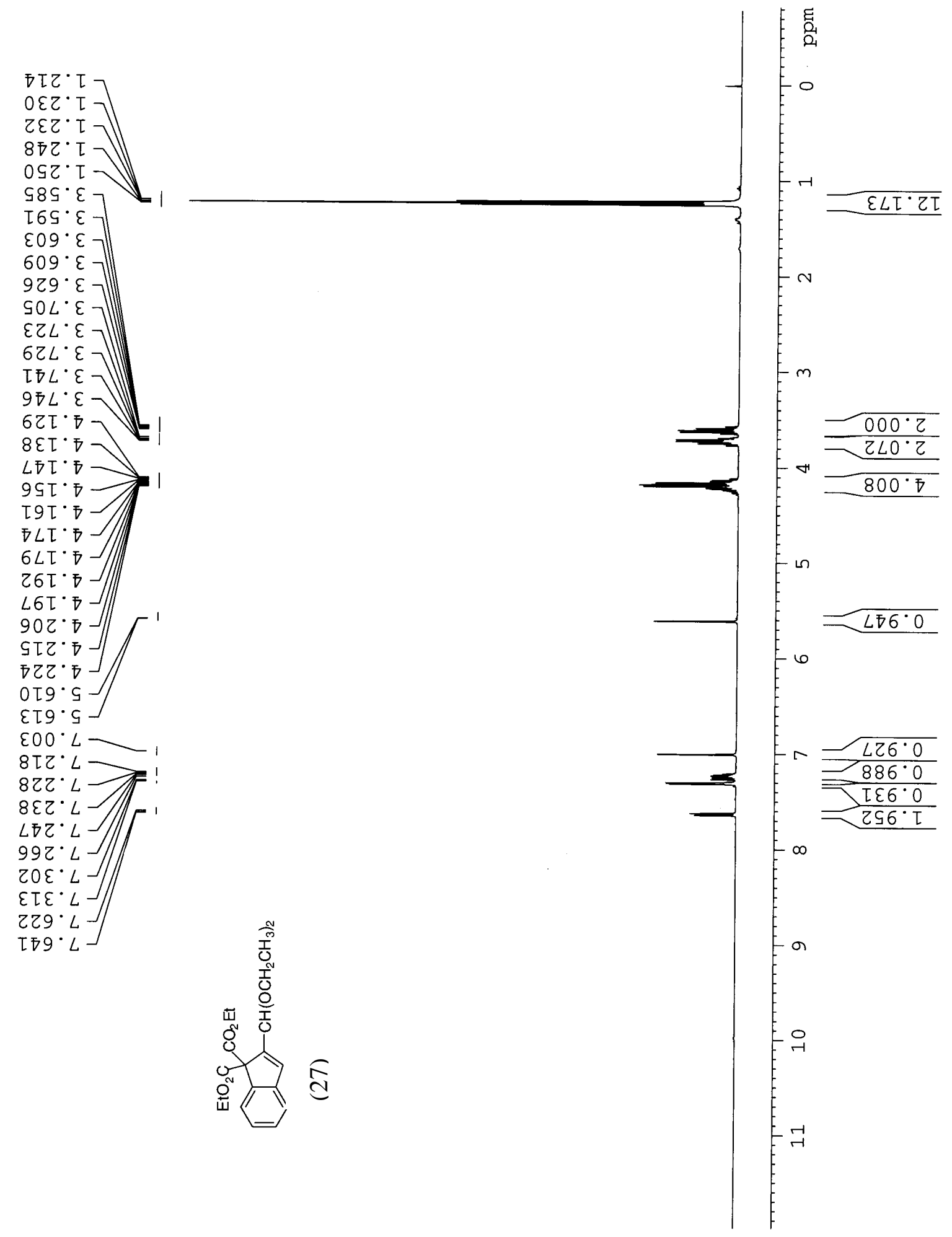


$0 \nabla 6^{\circ} \varepsilon \tau$

$\varsigma L Z \cdot S T$

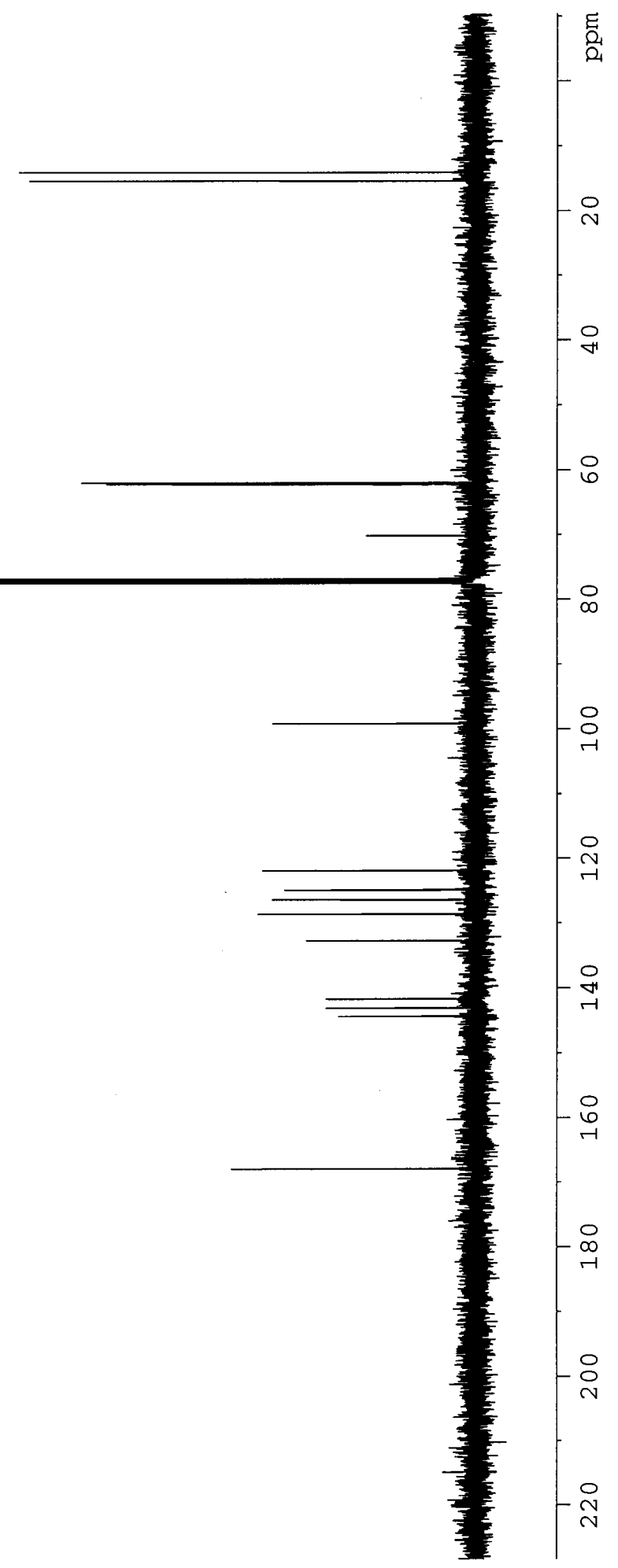

S๐8 T2T

8५8

26ह.9ZT

L8S.8ZT

โโL $2 \varepsilon \tau$

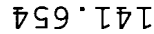

ธऽ० हธโ

8乙ย

$\varepsilon \varepsilon 6^{\circ} \angle 9 T$

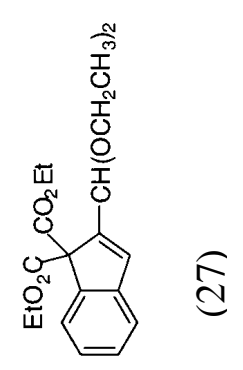

ำ 


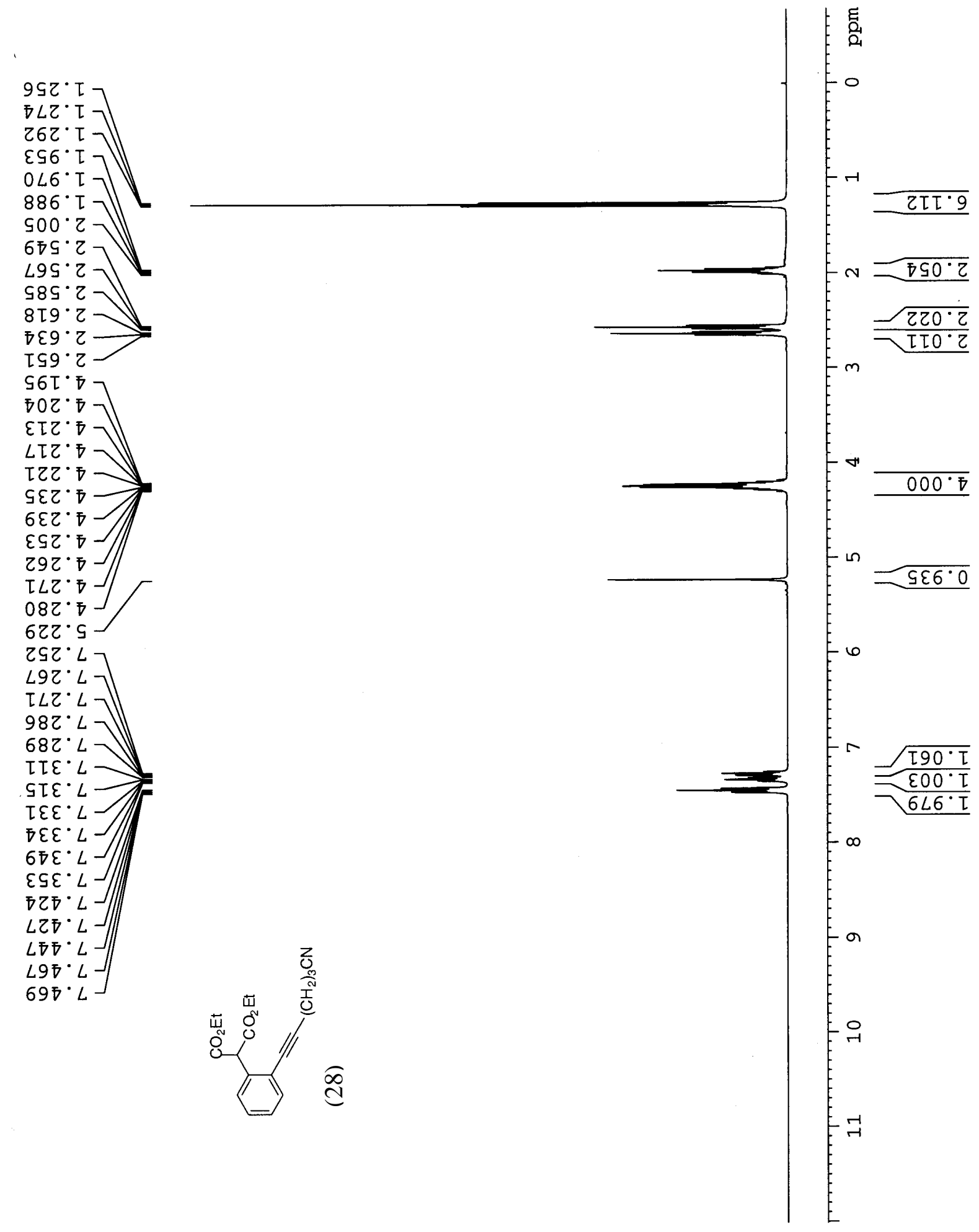


$\angle 60^{\circ} \mathrm{DT}$

$\mathrm{DZZ} \cdot 9 \mathrm{I}$

$569 \cdot 8 \mathrm{I}$

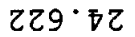

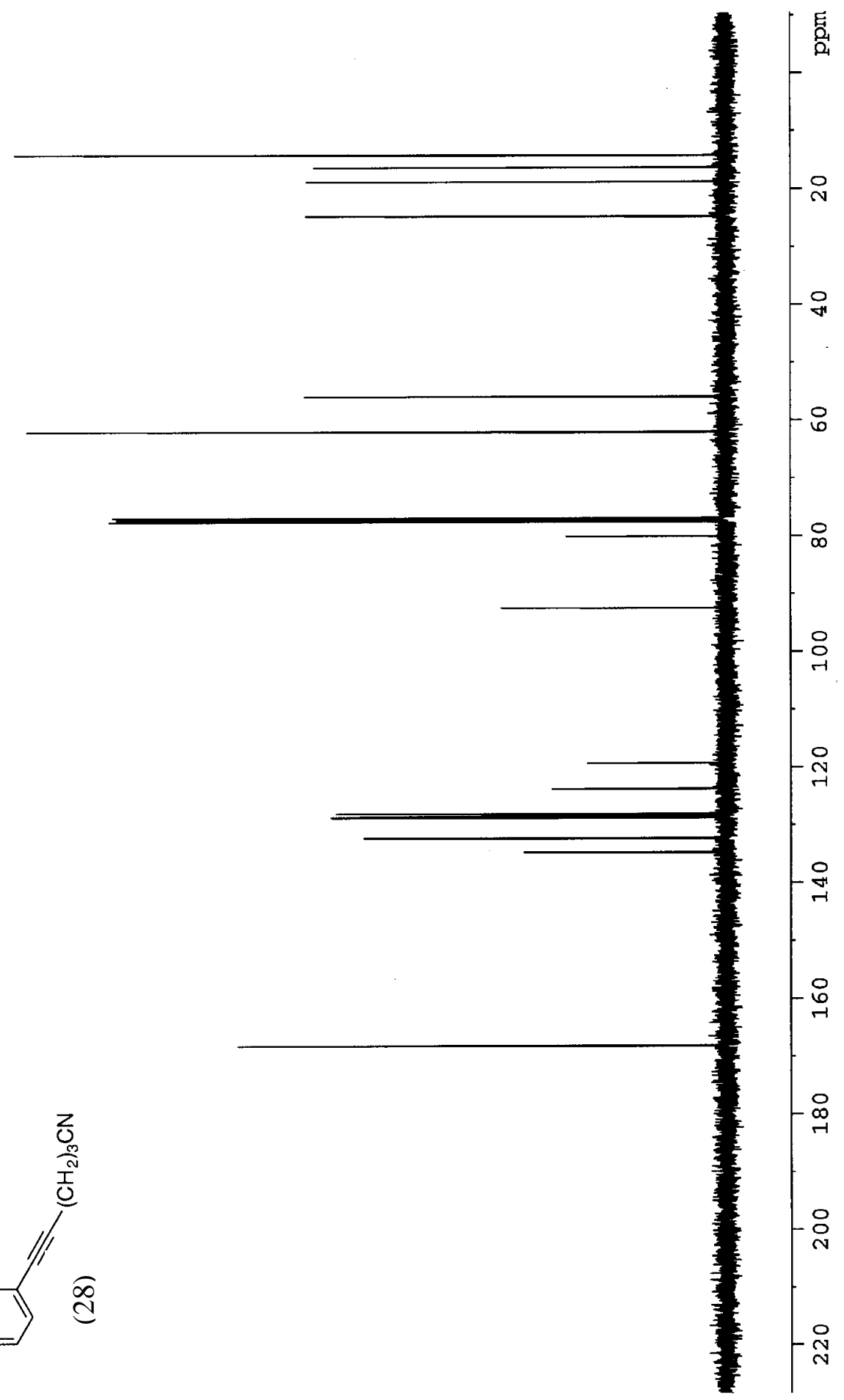

$58 I \cdot 6 I \tau-$

$\angle \angle 9^{\circ} \varepsilon Z T$

ธ86. $\angle Z T$

ฤฤ $8 Z T$

$Z \tau L \cdot 8 Z \tau$

$8 Z Z \cdot 乙 \varepsilon \tau$

$989^{\circ}$ ТEI -

$\mathrm{G} 80 \cdot 89 \tau$

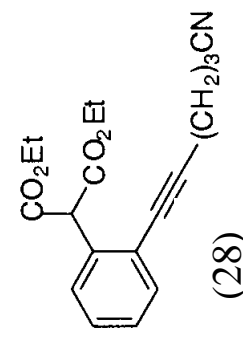




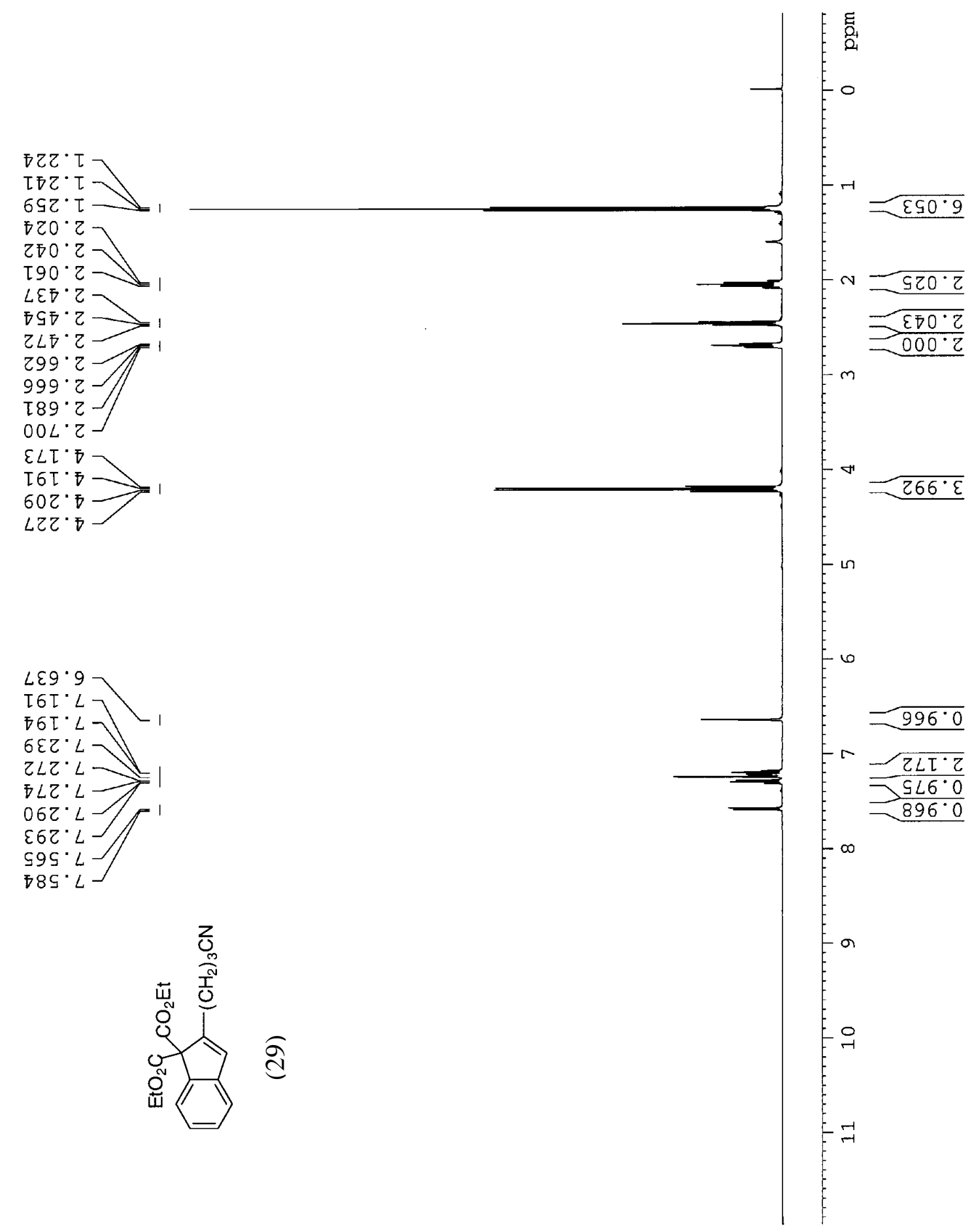




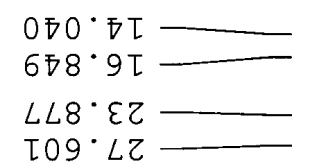

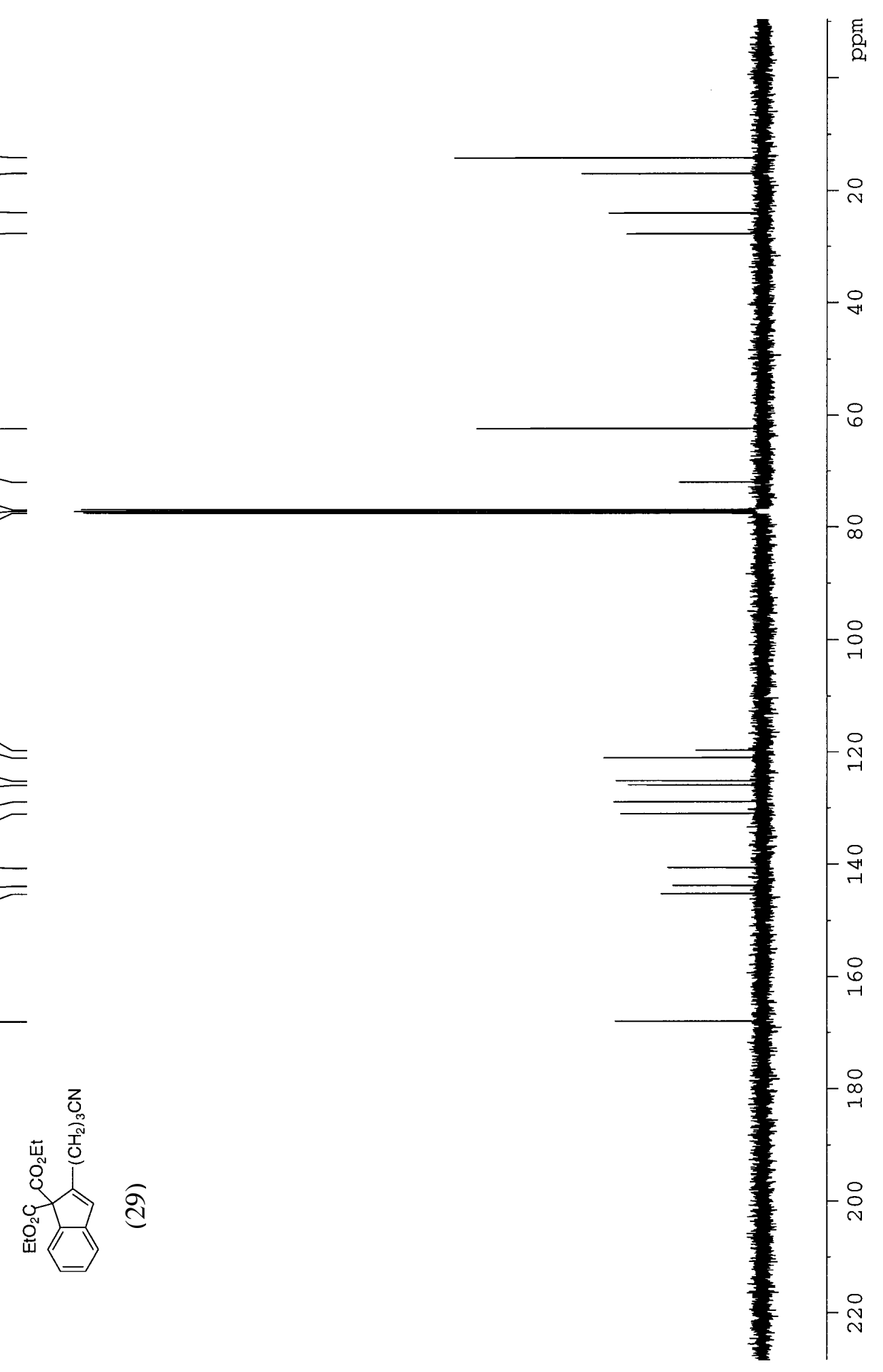

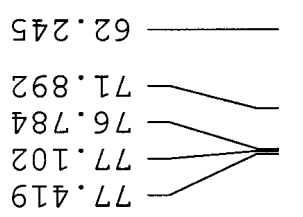

$\varepsilon I 9^{\circ} 6 \tau I$

ธ乙6. 0 Z

$0 \subseteq 0.5 Z T$

$\angle Z 8^{\circ} \mathrm{SZI}$

$06 L^{\circ} 8 Z T$

$806^{\circ} 0 \varepsilon I$

จ $6 \bar{\nabla}^{\circ} 0 \bar{I}$

$\angle 99^{\circ} \varepsilon \nabla \tau$

ऽ $60^{\circ} \varsigma \nabla \tau$

$\varepsilon 06^{\circ} \angle 9 I$

\section{颔}




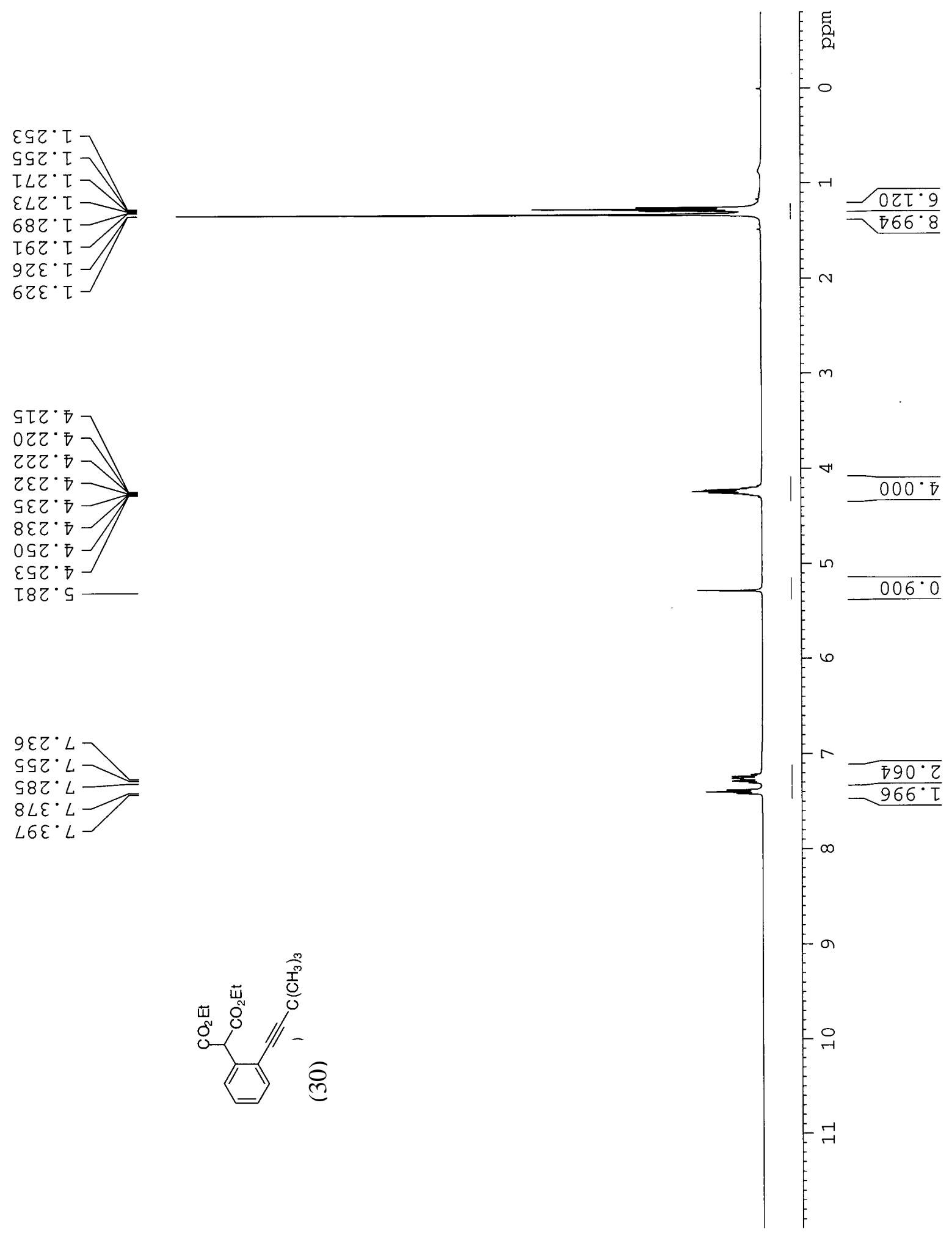


OOT' TI

$\varepsilon z Z \cdot 8 Z$

$9 \varepsilon \sigma^{\circ} \circ$

$088 \cdot 59$

ZLL T9

$56 L \cdot 9 L=$

हIT $L L$

TET $L$

$8 \varepsilon 0^{\circ}$ TOL

$890^{\circ}$ 万ZT

I8 ${ }^{\circ} \angle Z T$

Z५ $8 \mathrm{8ZT}$

9२8 โहL

乙LL' TEL

$89 \varepsilon^{\circ} 89 I$

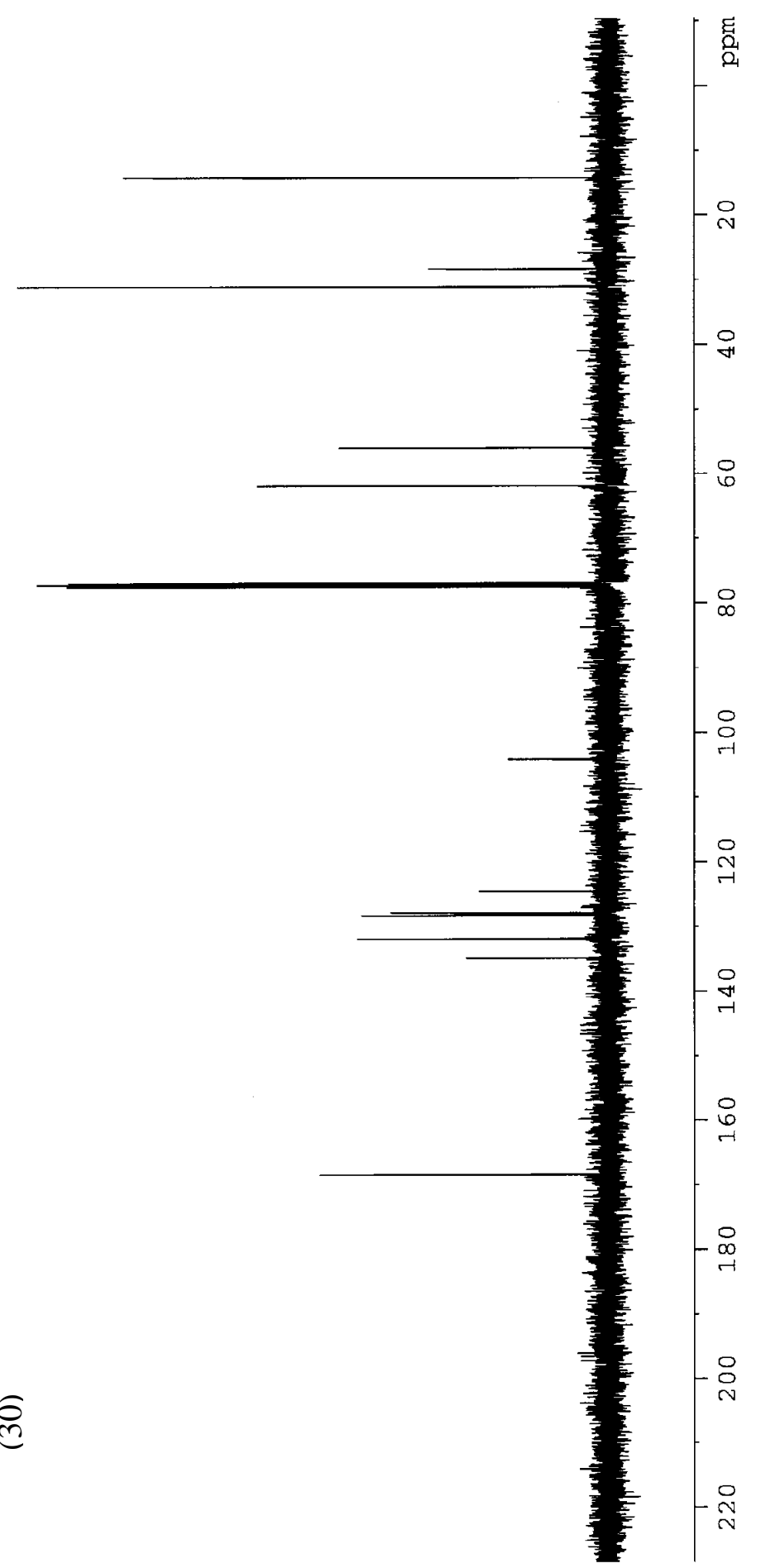




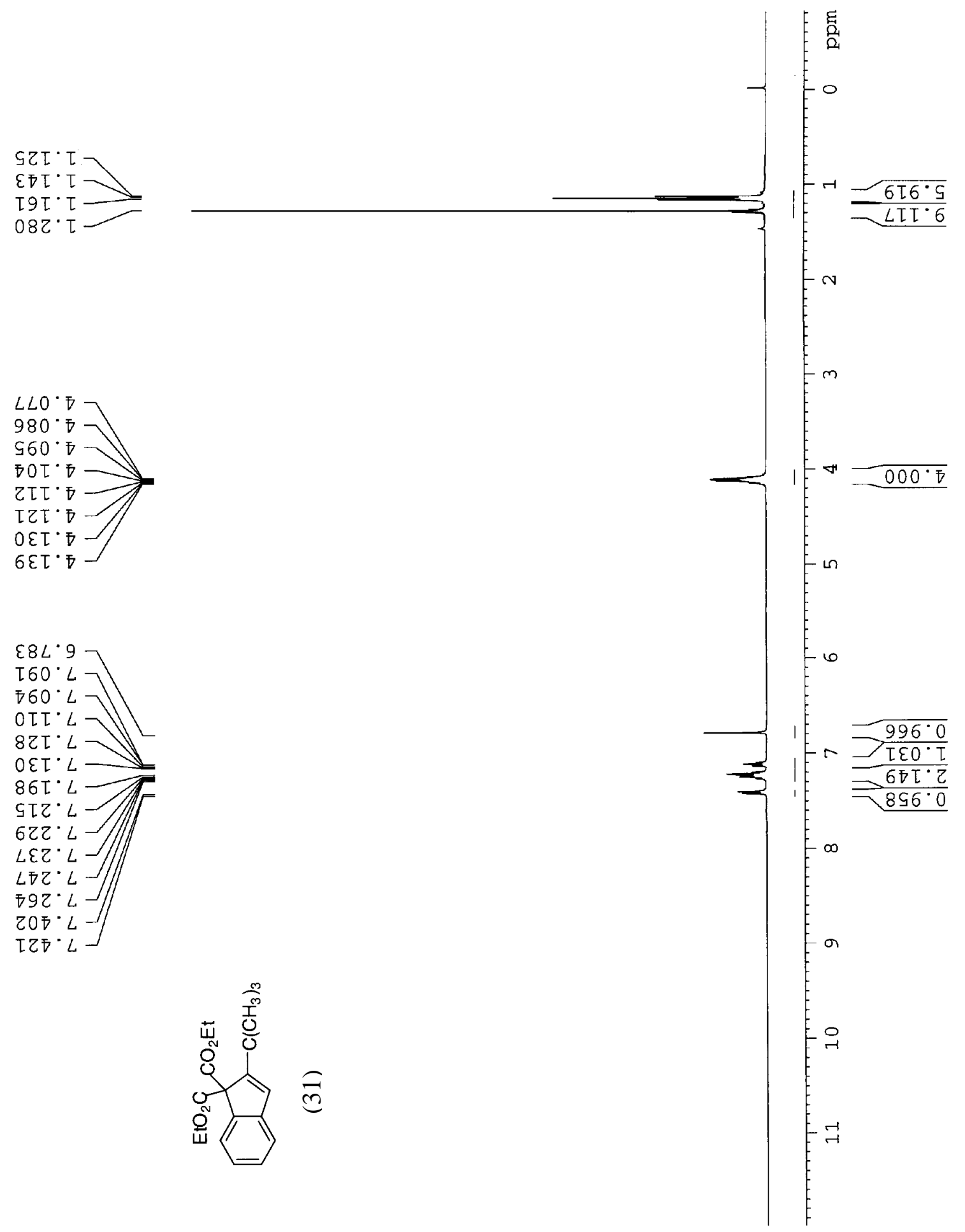




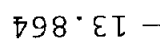

$\tau 6 \tau \cdot \tau \varepsilon=$

$6 L L \cdot T \varepsilon$

8 โ9. 29

$009^{\circ} 0 L$

$0 L L \cdot 9 L$

$880^{\circ} \mathrm{LL}$

$900^{\circ} \angle L$

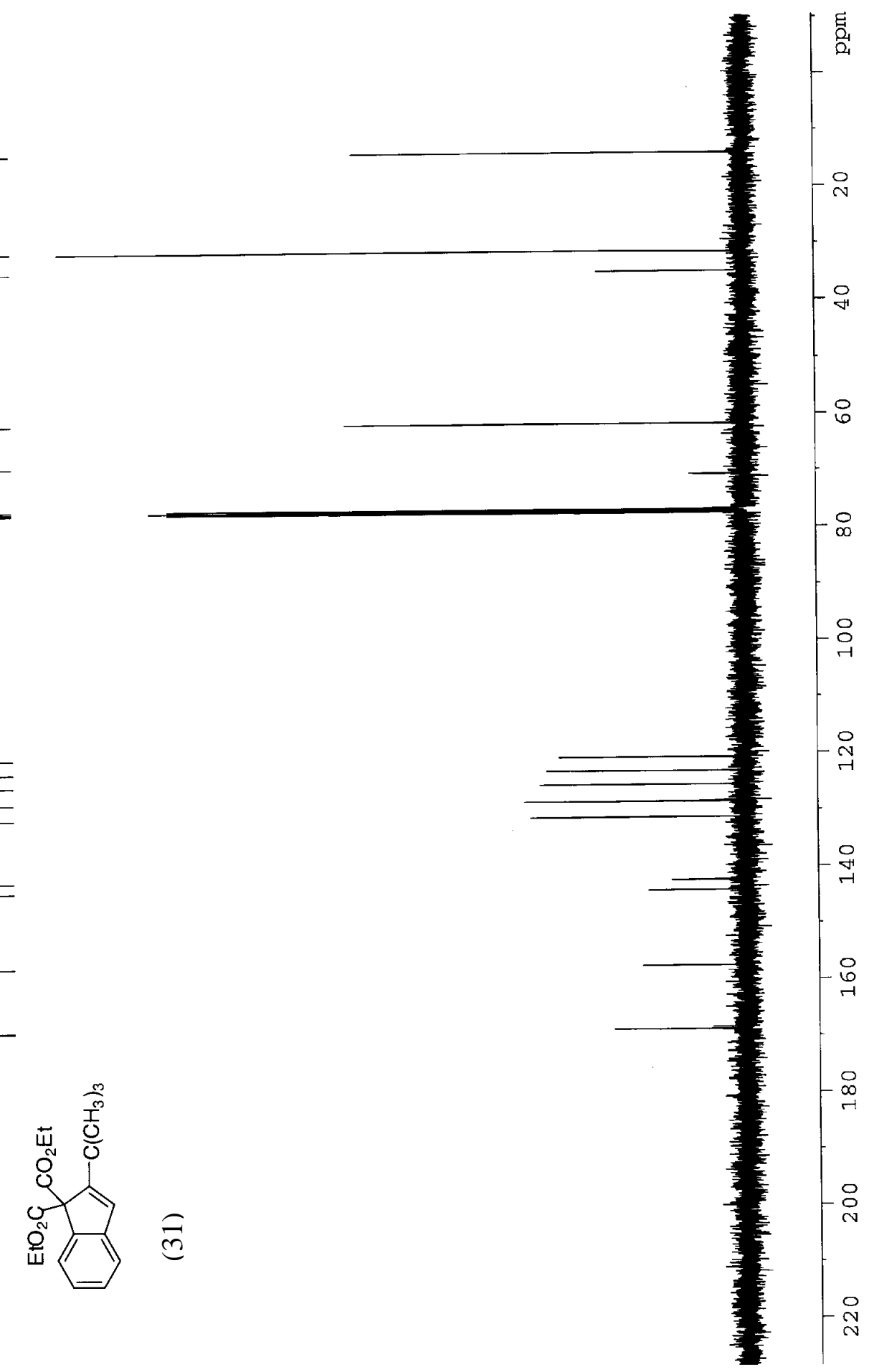

$\angle \varepsilon 9^{\circ} \mathrm{OZT}$

$080^{\circ} \varepsilon Z \mathrm{Z} \longrightarrow$

โ๐๊.รZT

$8 Z{ }^{\circ} 8 Z T$

$9 L \tau \cdot \tau E \tau-$

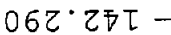

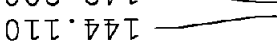

$S L \bar{C} \angle S T$

$\angle 9 L \cdot 89 I$ 


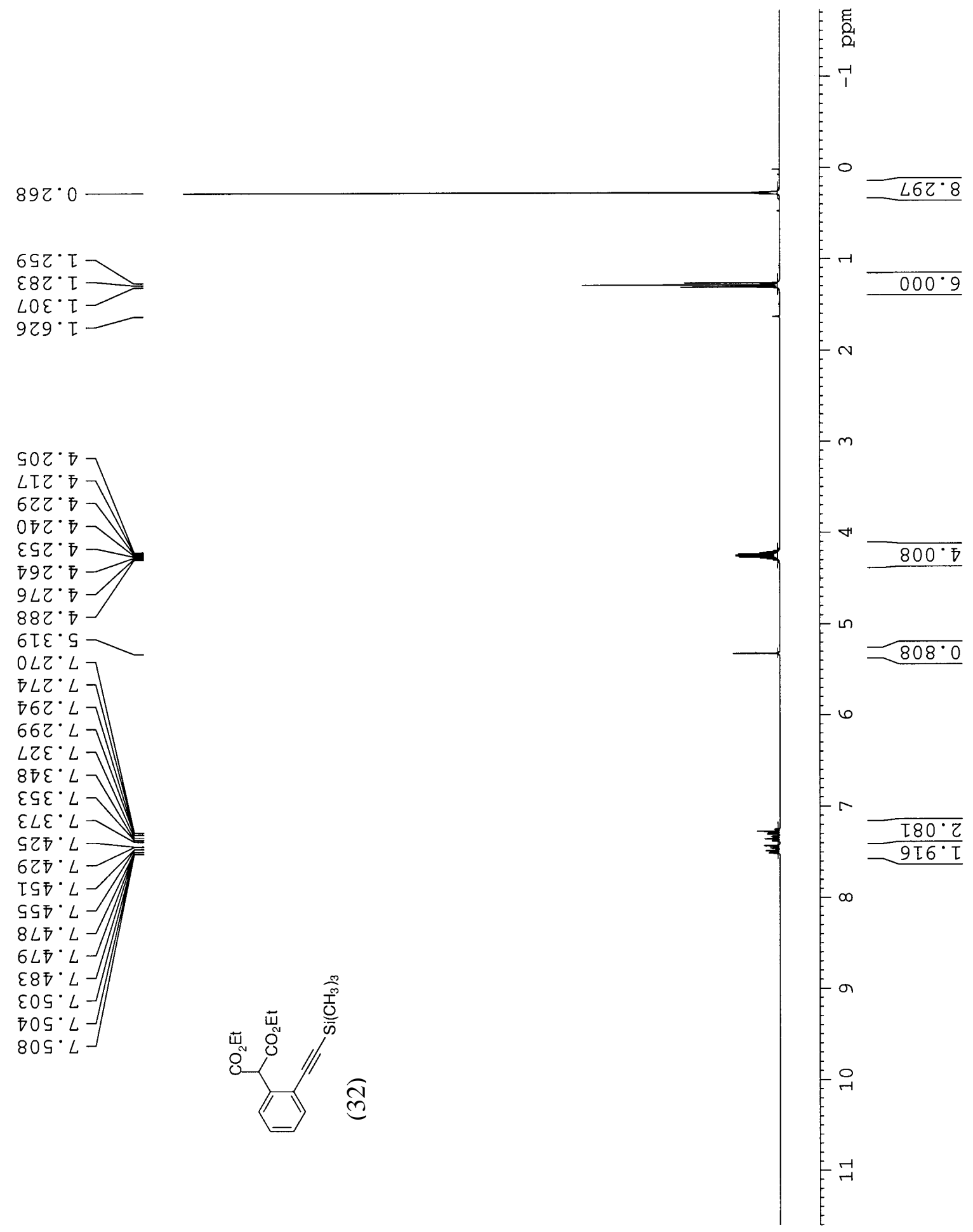




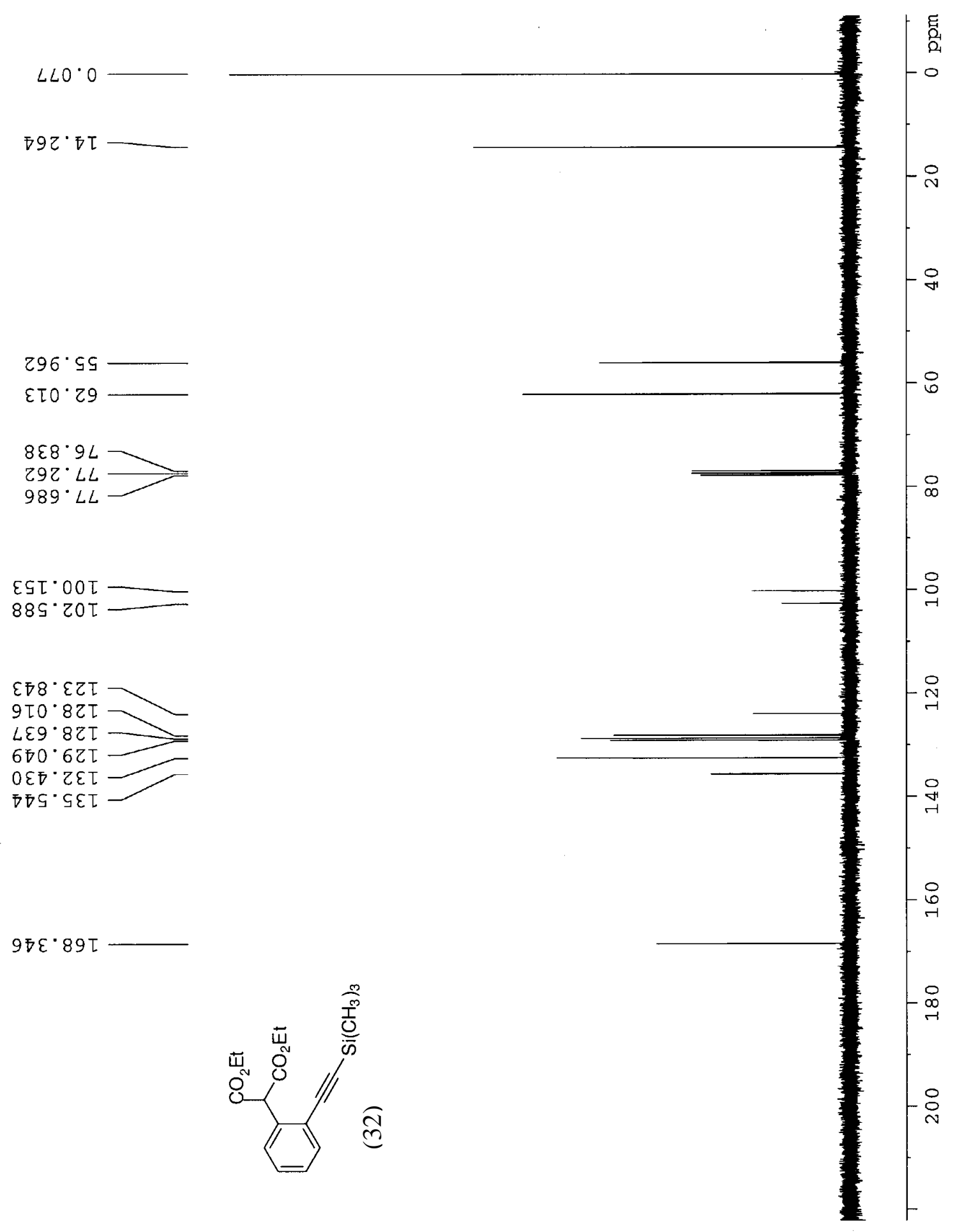




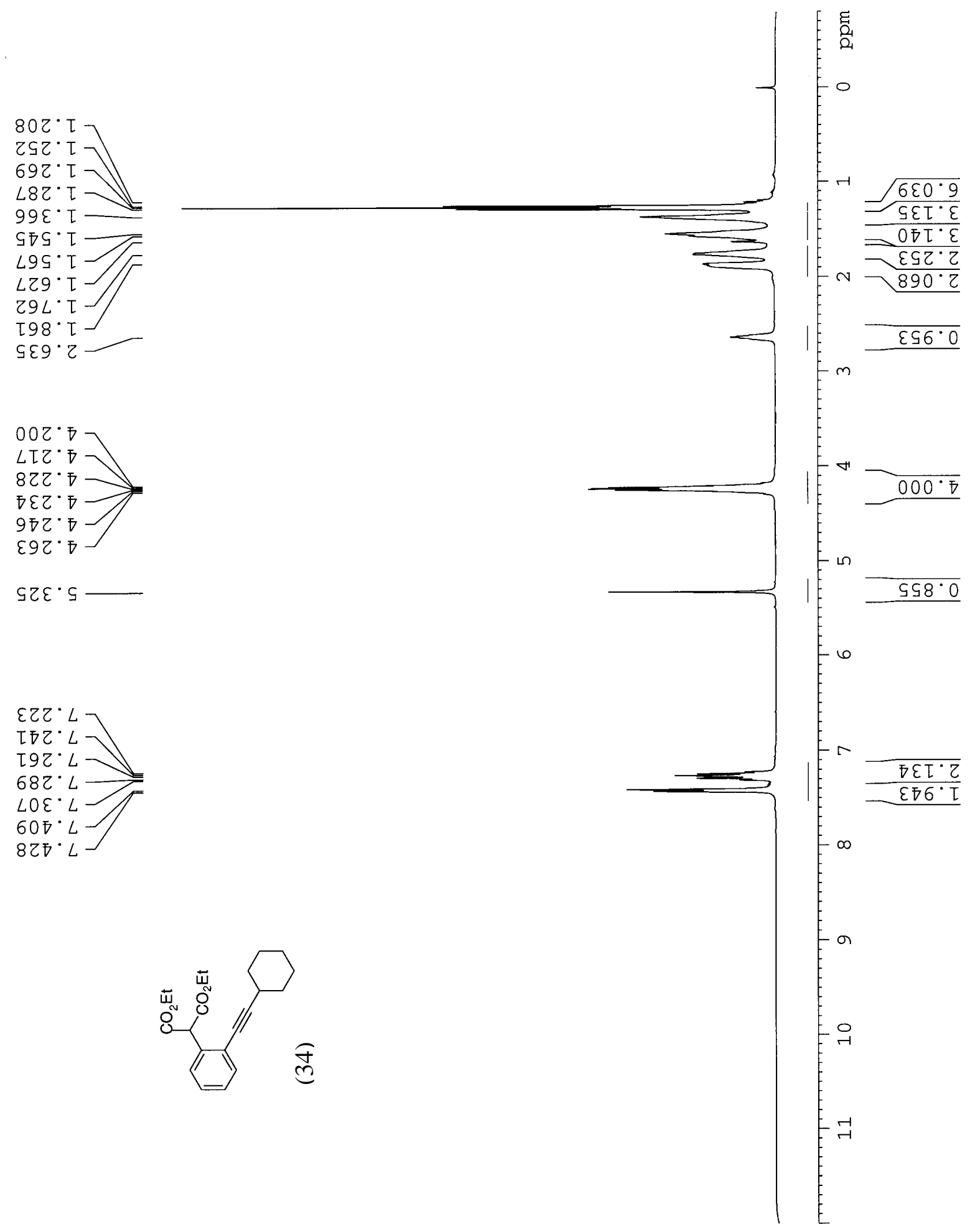



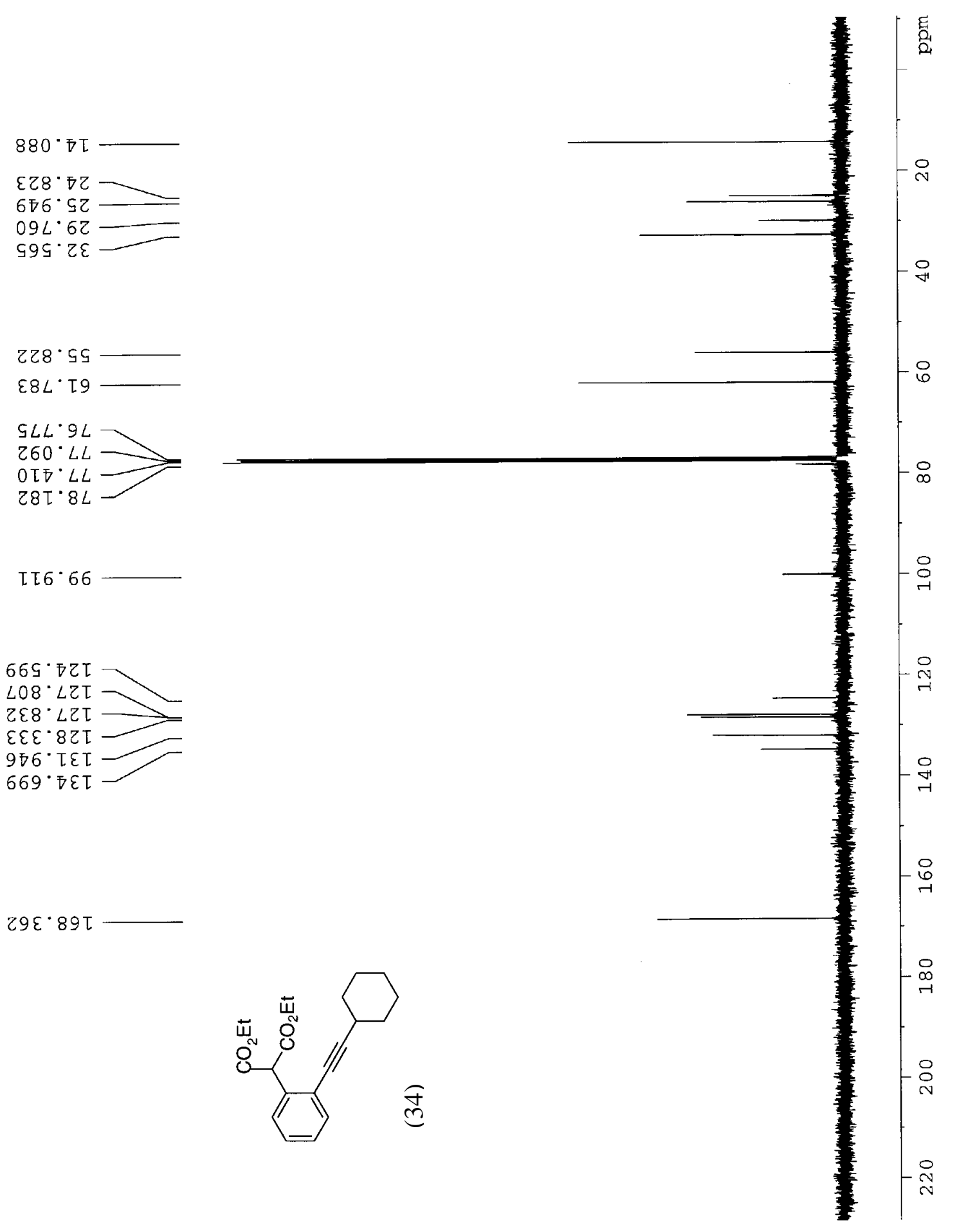

$669^{\circ}$ จ2

$\angle 08^{\circ} \angle Z T$

zह8. LZL $\longrightarrow$

$9 \varpi \sigma^{\circ} \mathrm{T} \varepsilon \mathrm{T}$

$669^{\circ}$ $\varepsilon \mathrm{I}$

$29 \varepsilon \cdot 89 \tau$ 


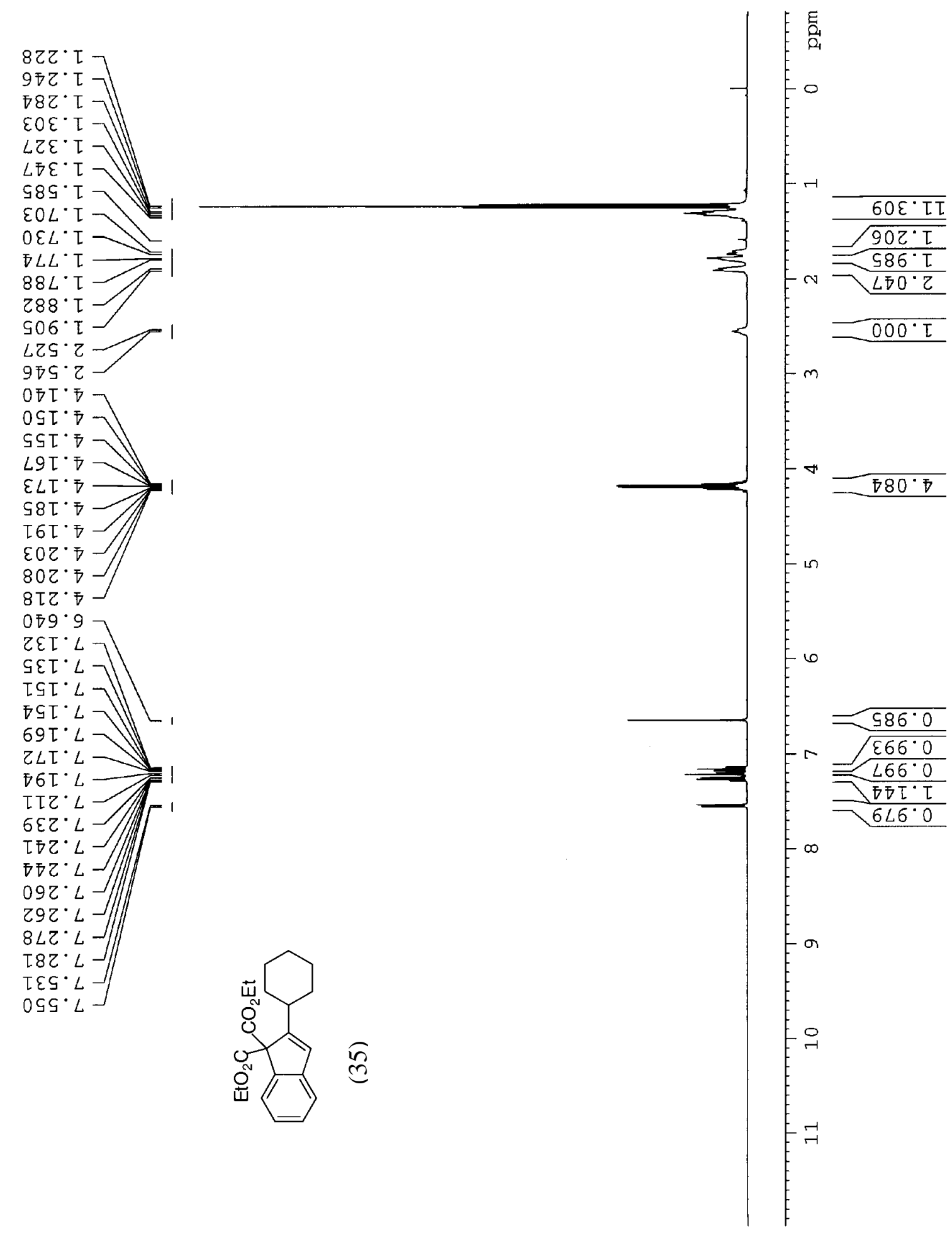



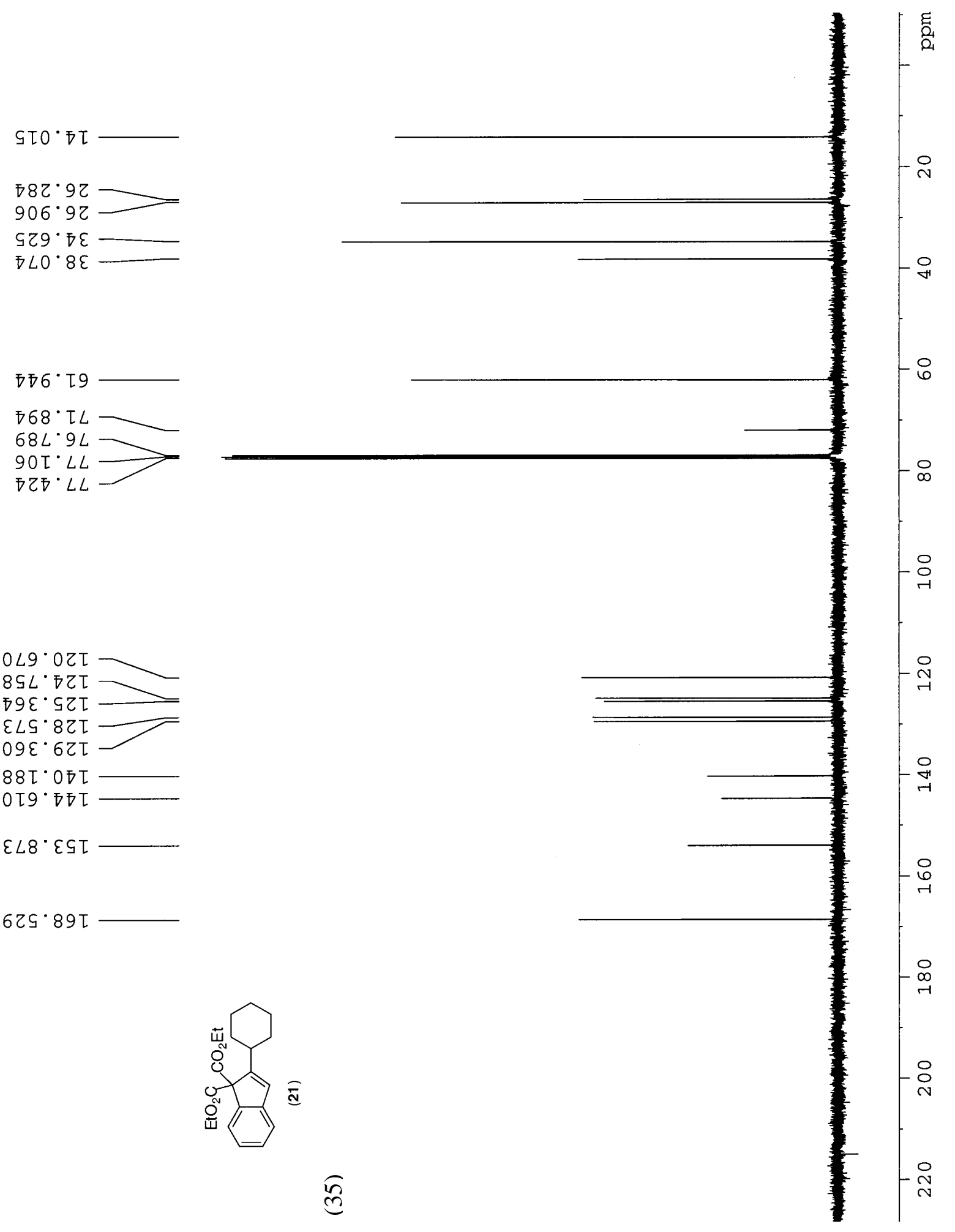

$0 \angle 9^{\circ} 0 Z T$

8SL ஏ乙L

ฤ $9 \varepsilon^{\circ} \varsigma 乙 \tau$

$\varepsilon \angle S^{\circ} 8 Z T$

$09 \varepsilon \cdot 6 Z \tau$

$88 \tau^{\circ} 0 \mathrm{TL}$

Oโ9

$\varepsilon\left\llcorner 8^{\cdot} \varepsilon \subseteq \tau\right.$

$629 \cdot 89 \tau$ 


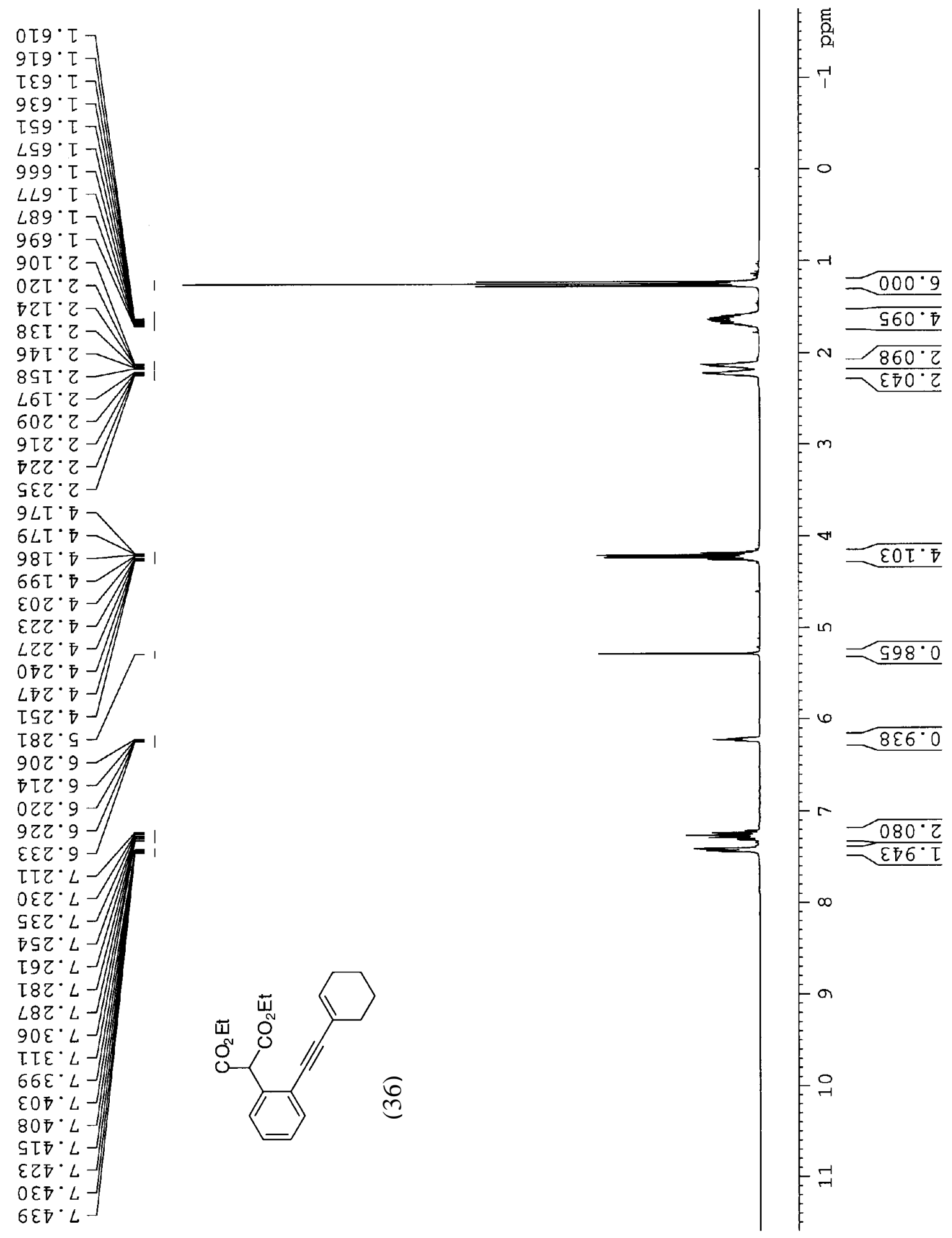




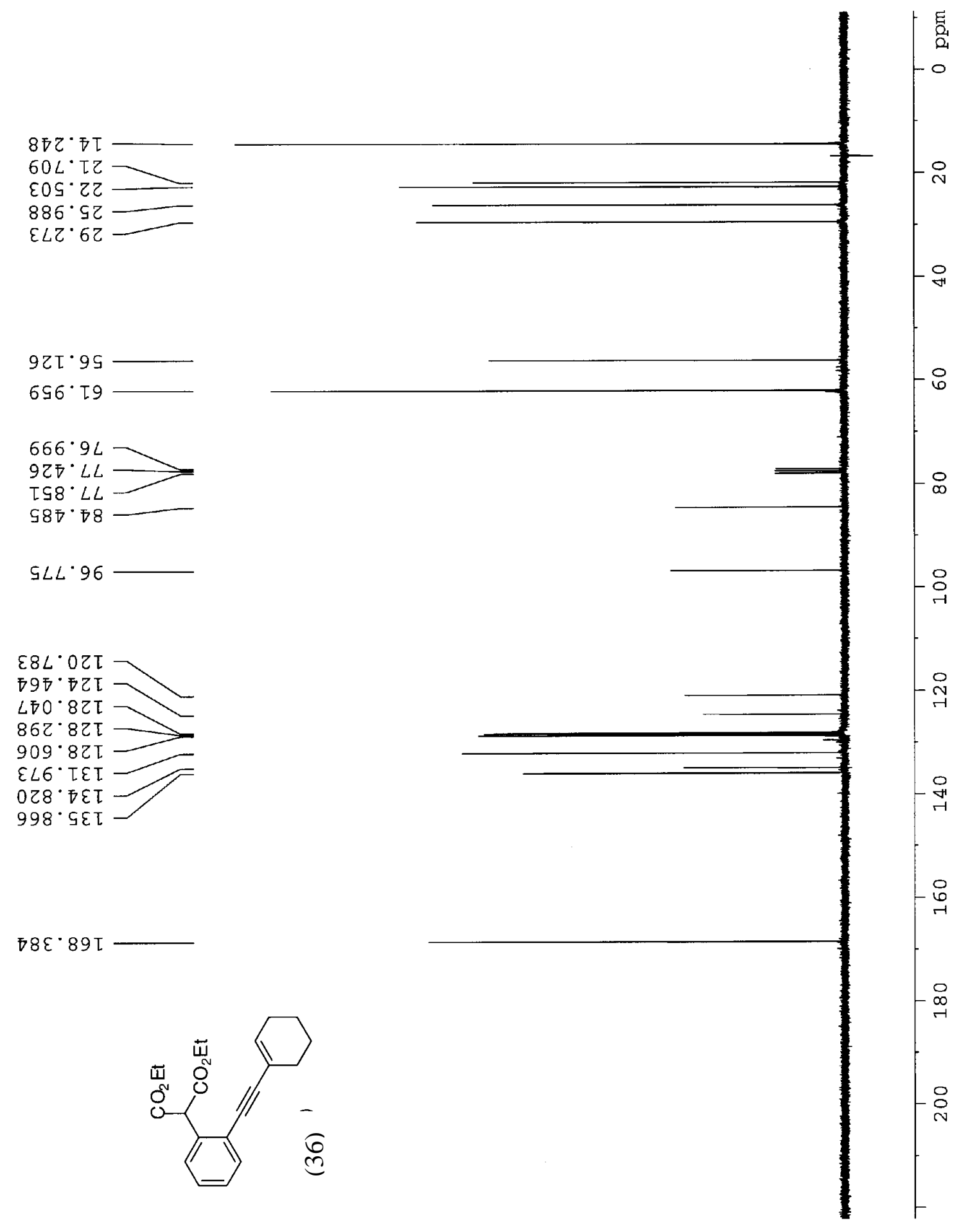




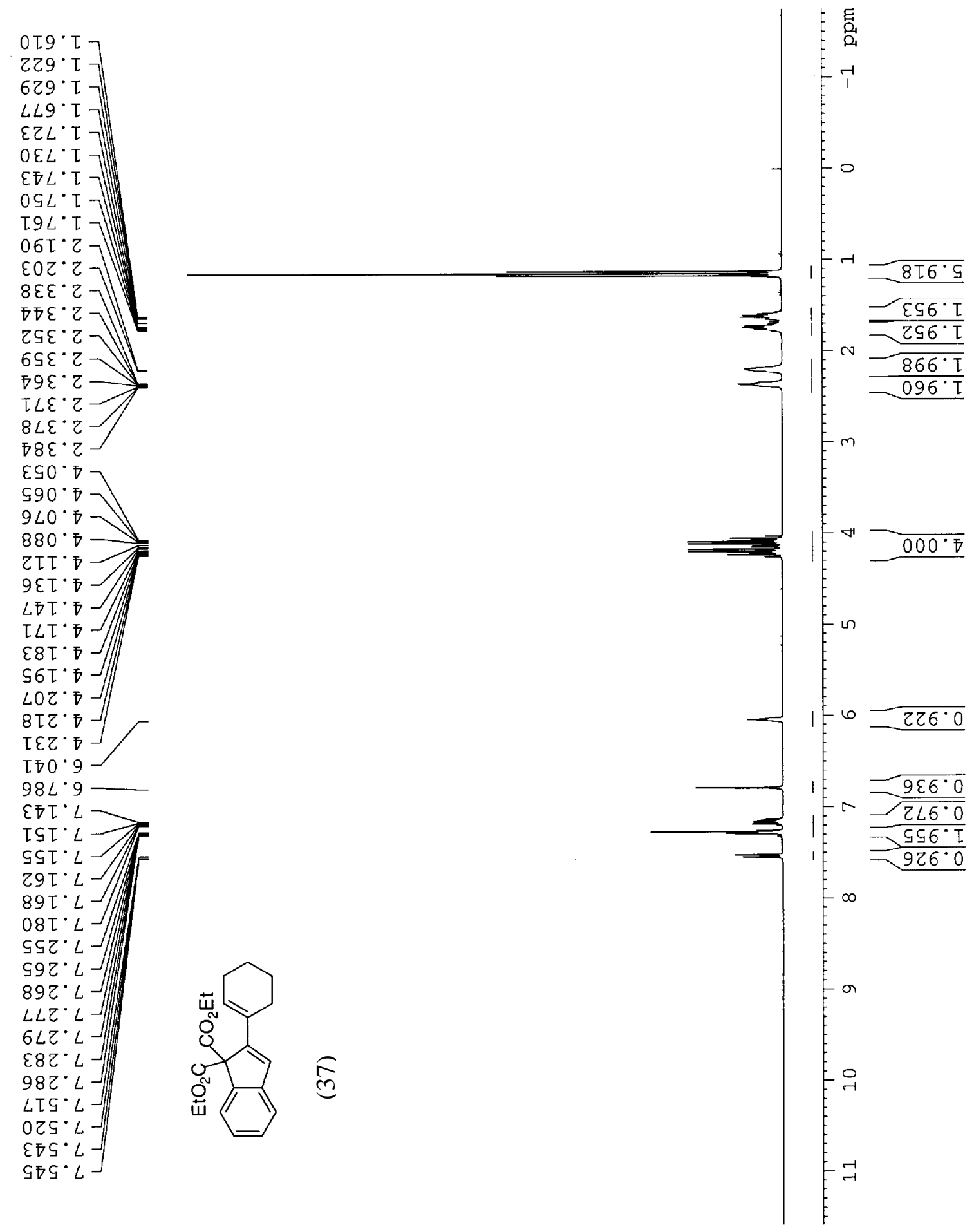


$676^{\circ} \varepsilon \tau$

$9 L L \cdot z Z-$

zoL.9Z

$\varepsilon \tau L \cdot 9 Z$

T89. $โ 9$

0षL.69

0ह8. $9 L$

$8 T^{\circ} \cdot L L$

$\angle 97^{\circ} \angle L$

ट89. हटT

$882 \cdot 8 Z T$

$969 \cdot 8 Z T$

万Z००6ZT

OZS OEL

ÐL䏝

6LI・市

$\varepsilon Z 6^{\circ} L \nabla \tau$

$899 \cdot 89 T$

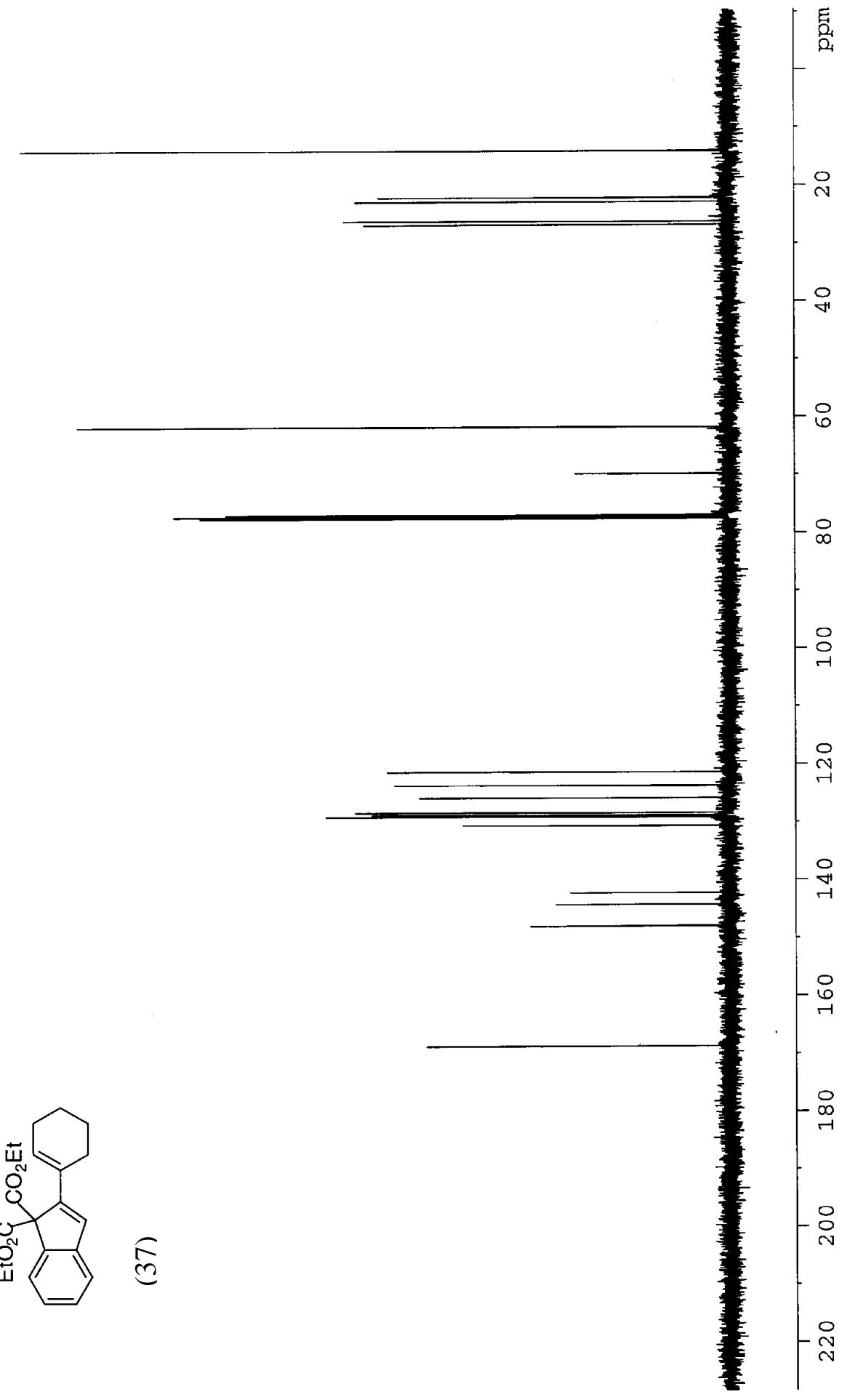




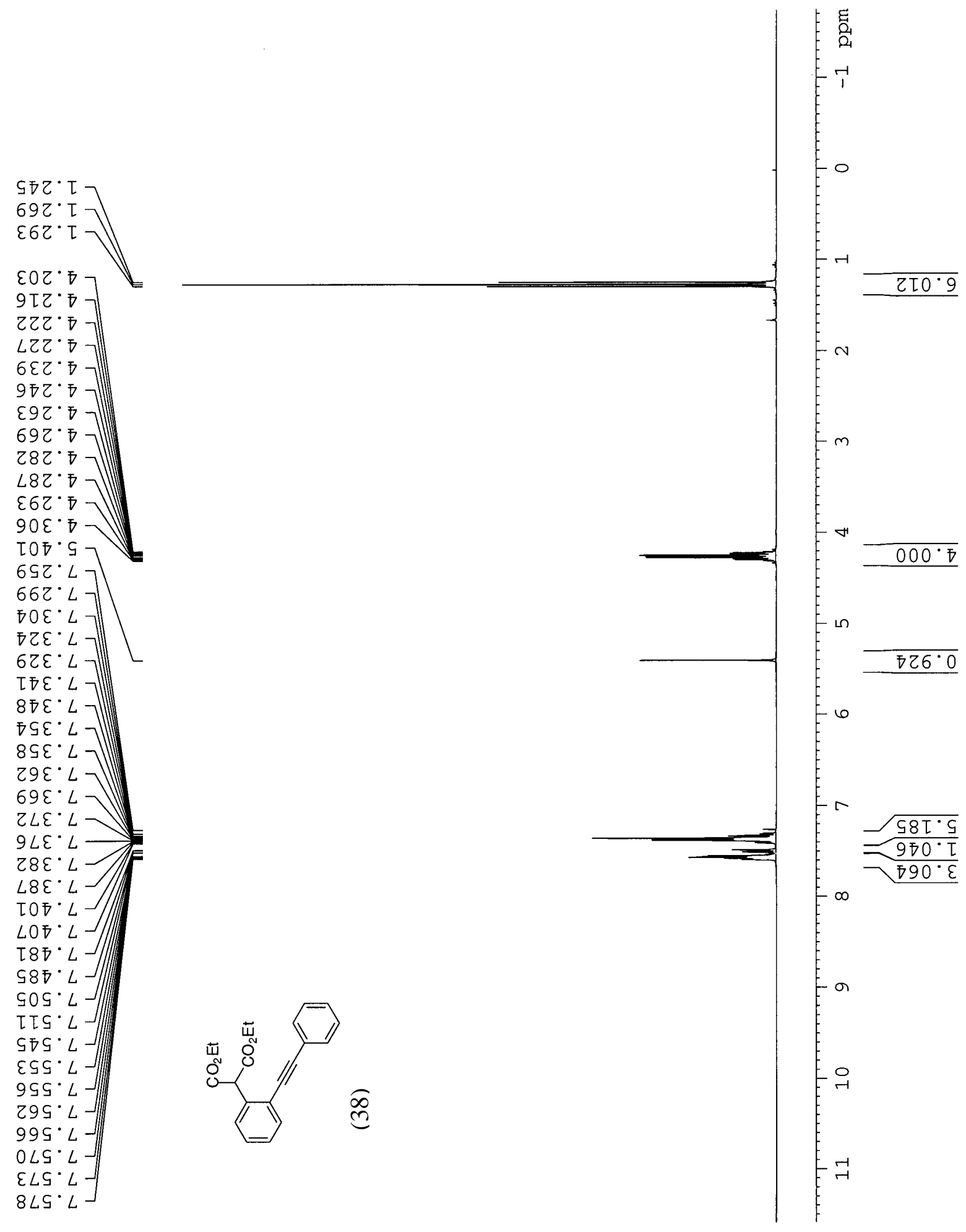


๑60・五

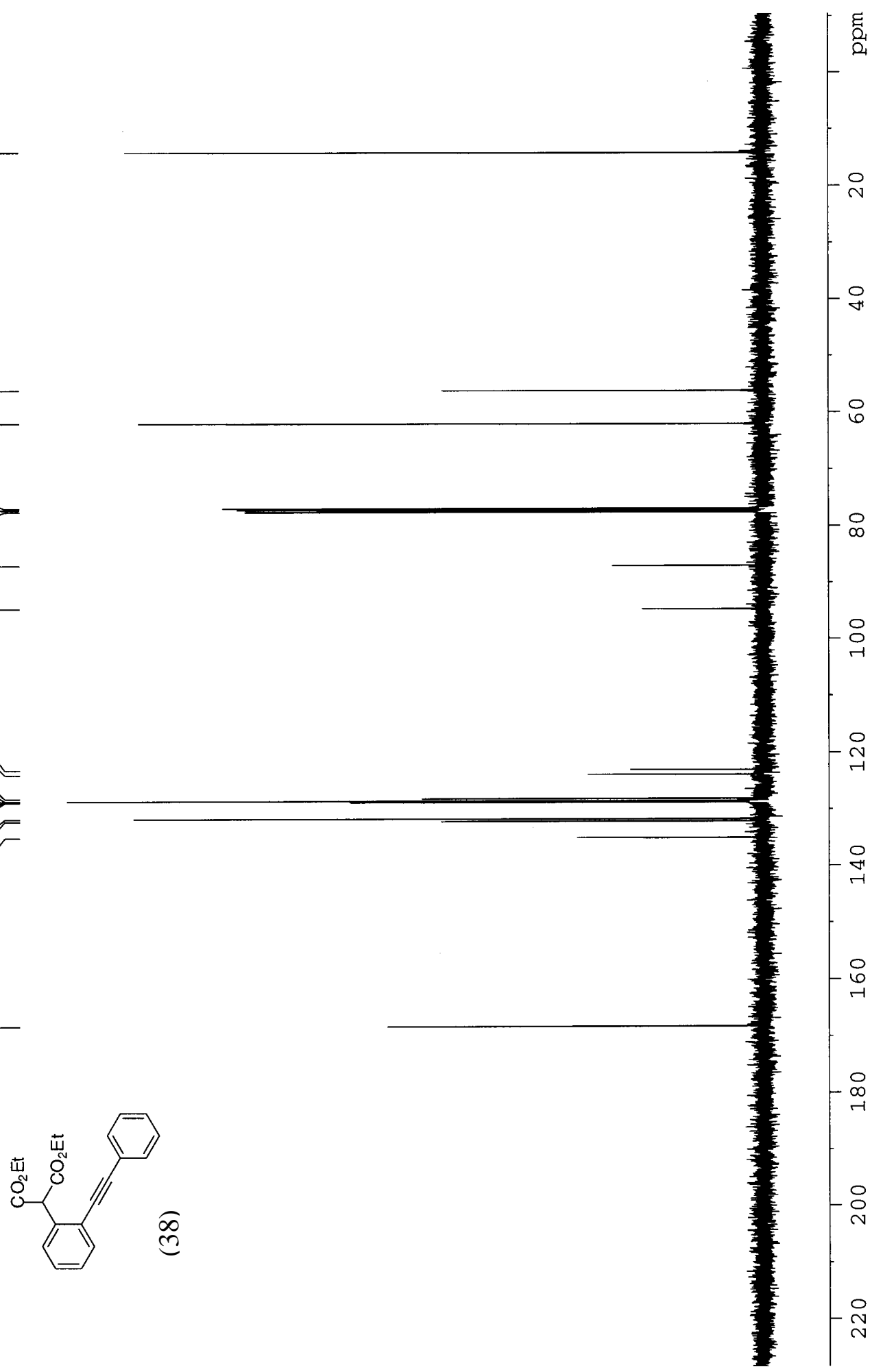

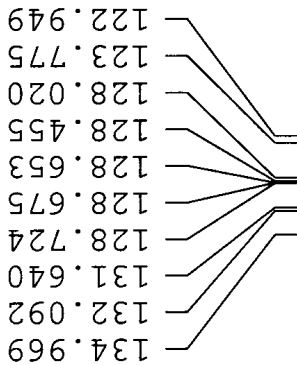

$\varepsilon Z Z \cdot 89 \tau$ 


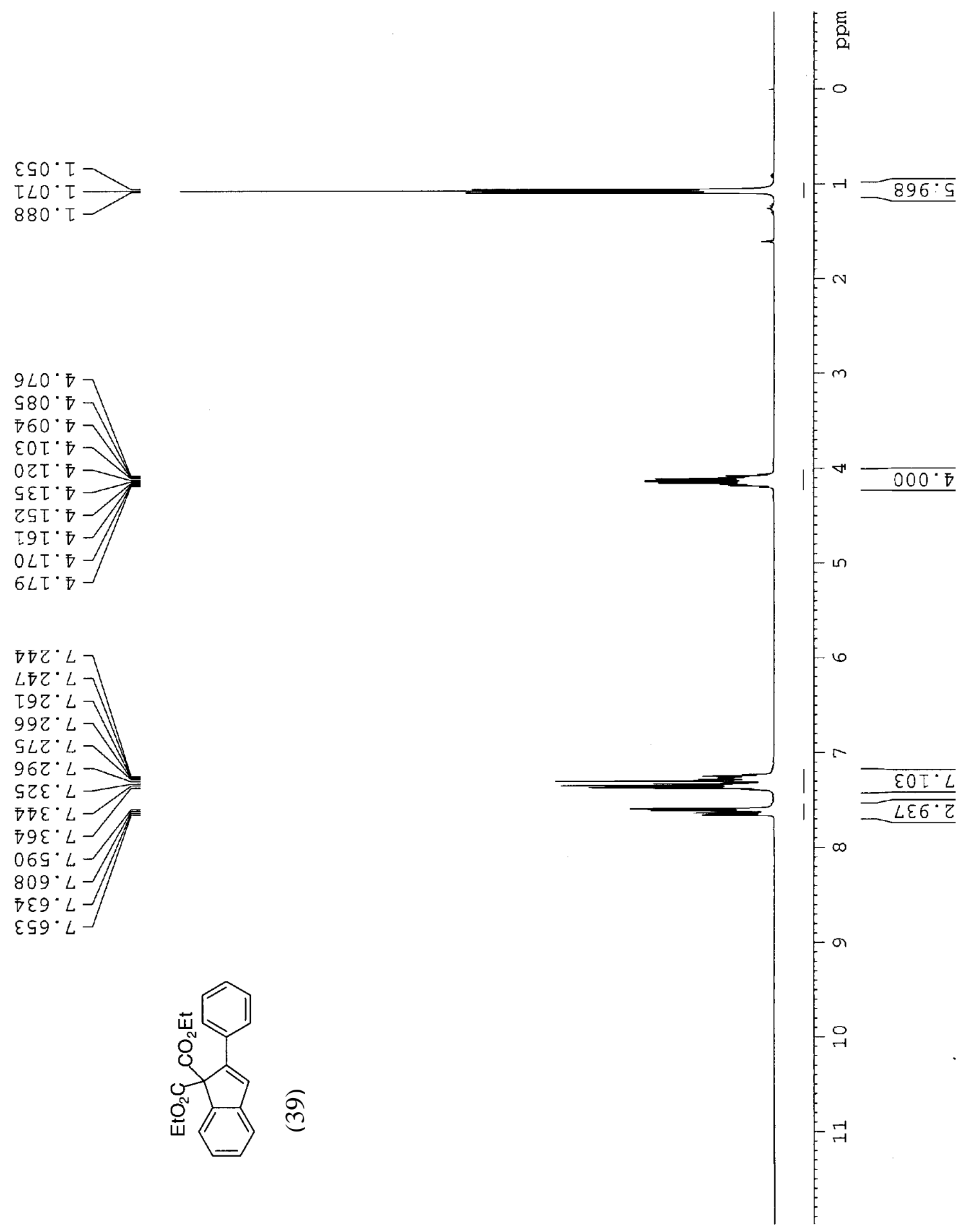


$8 \tau 8 \cdot \varepsilon \tau$

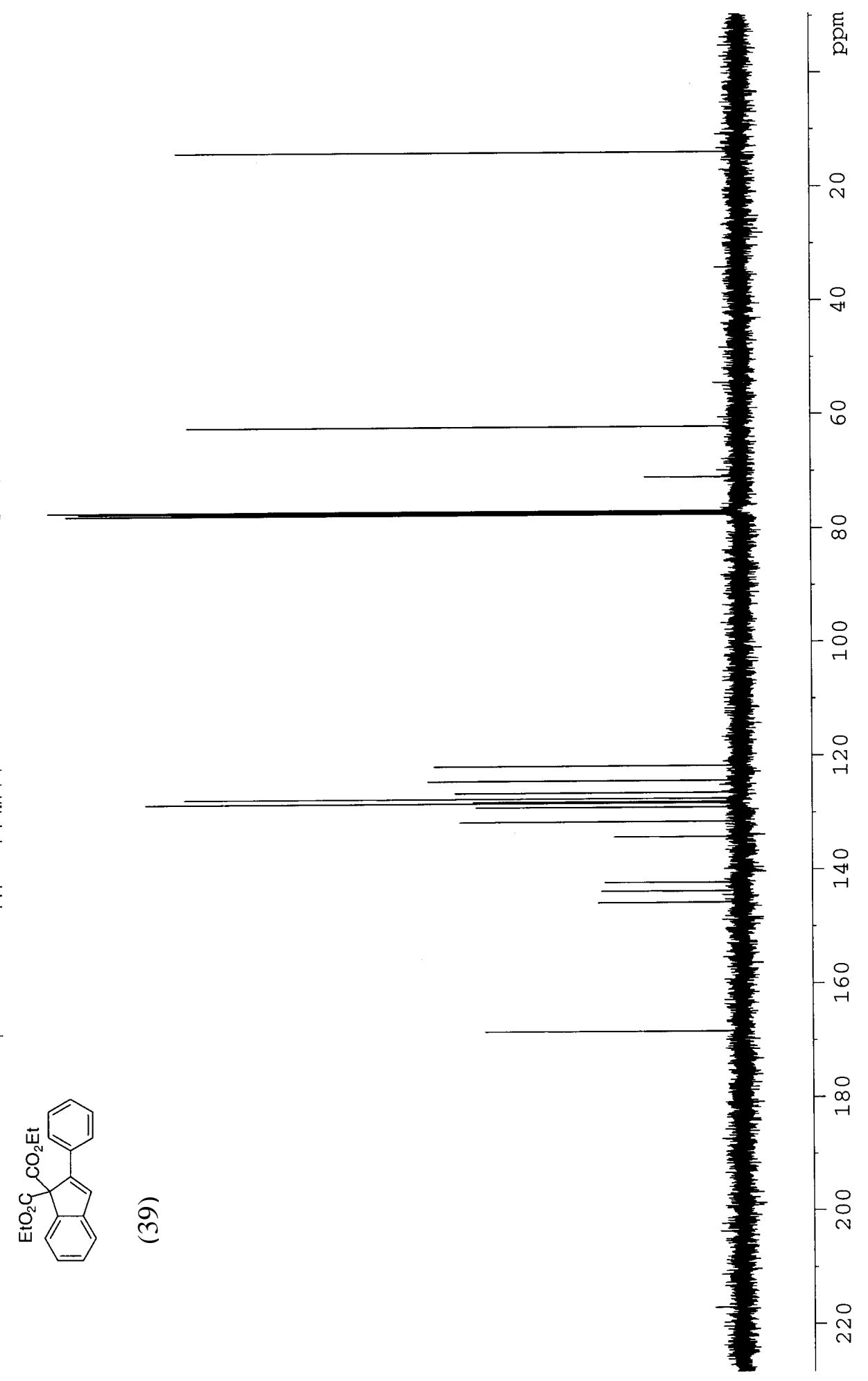

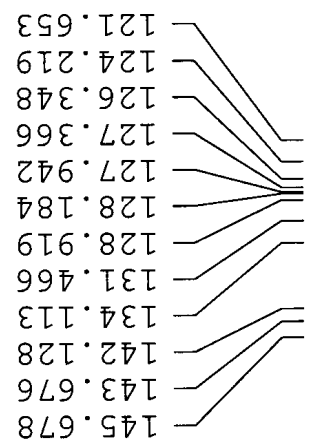

$\varepsilon L Z \cdot 89 I$

290.29

$508^{\circ} 9 L$

$\varepsilon Z T^{\circ} L L$

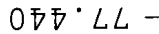

\section{展}

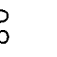




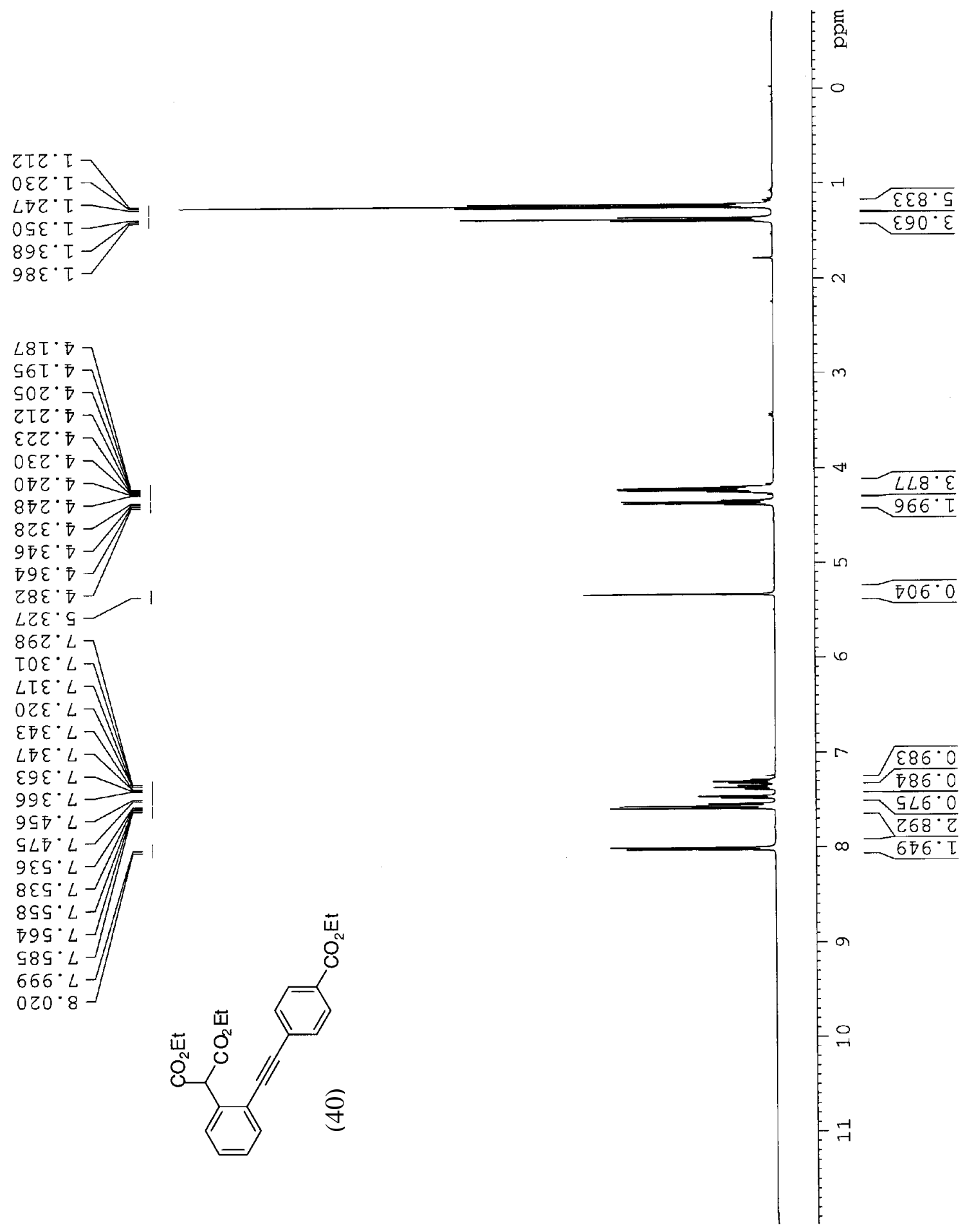


$\tau 80^{\circ} \bar{\square}$

$\varepsilon \varpi \tau \cdot 95$

OहZ ' I9

น86. โ9

$278 \cdot 9 L$

$09 T^{\circ} L L$

$8 \angle T^{\circ} \angle L$

$8 \tau L \cdot 68$

$\varepsilon 8 L^{\circ} \varepsilon \sigma$

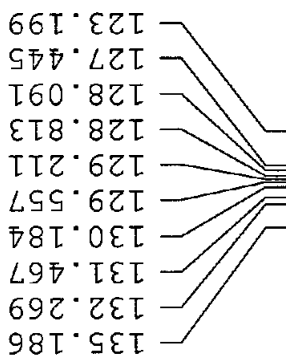

ZT0.99T

$870 \cdot 89 \mathrm{I}$

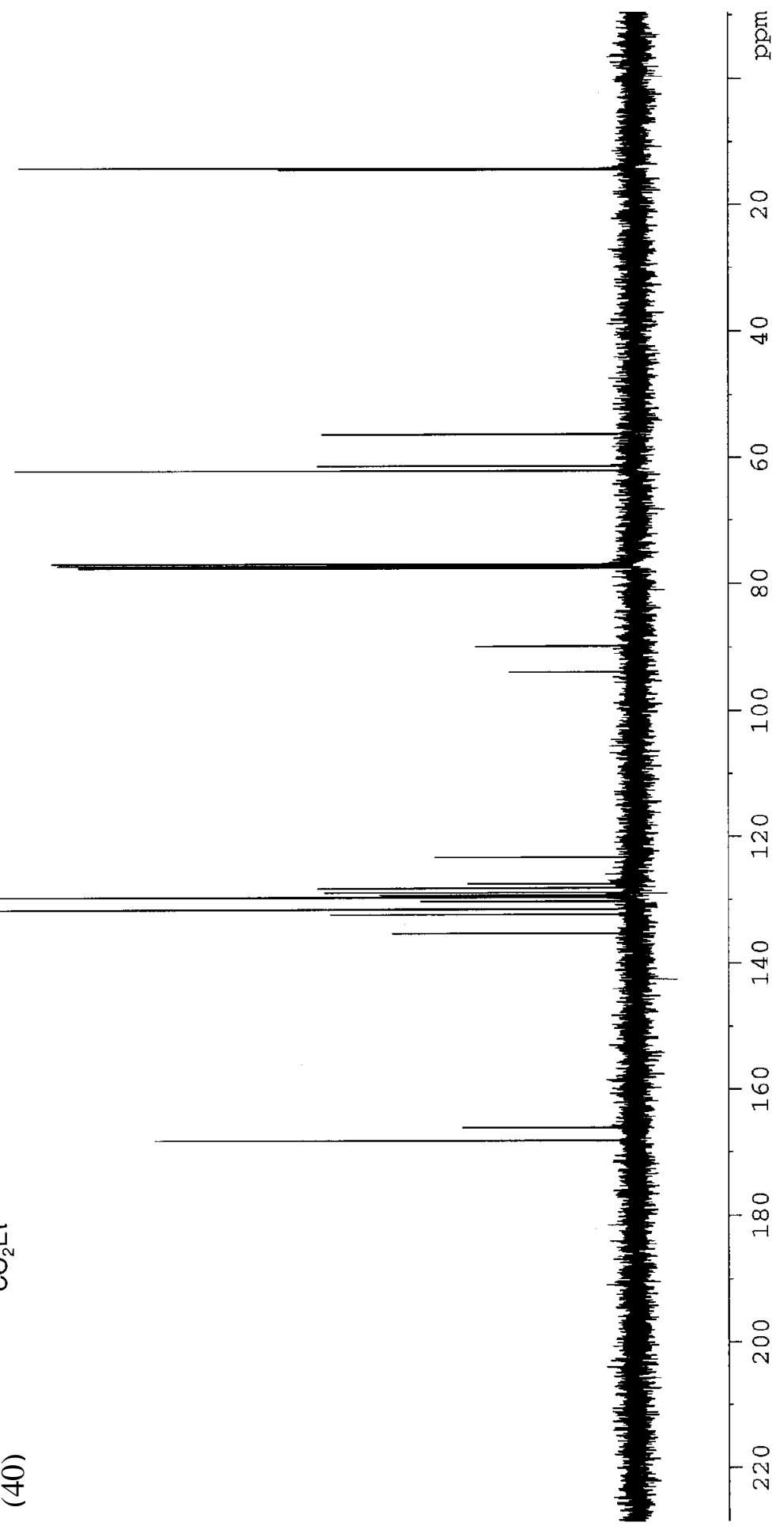




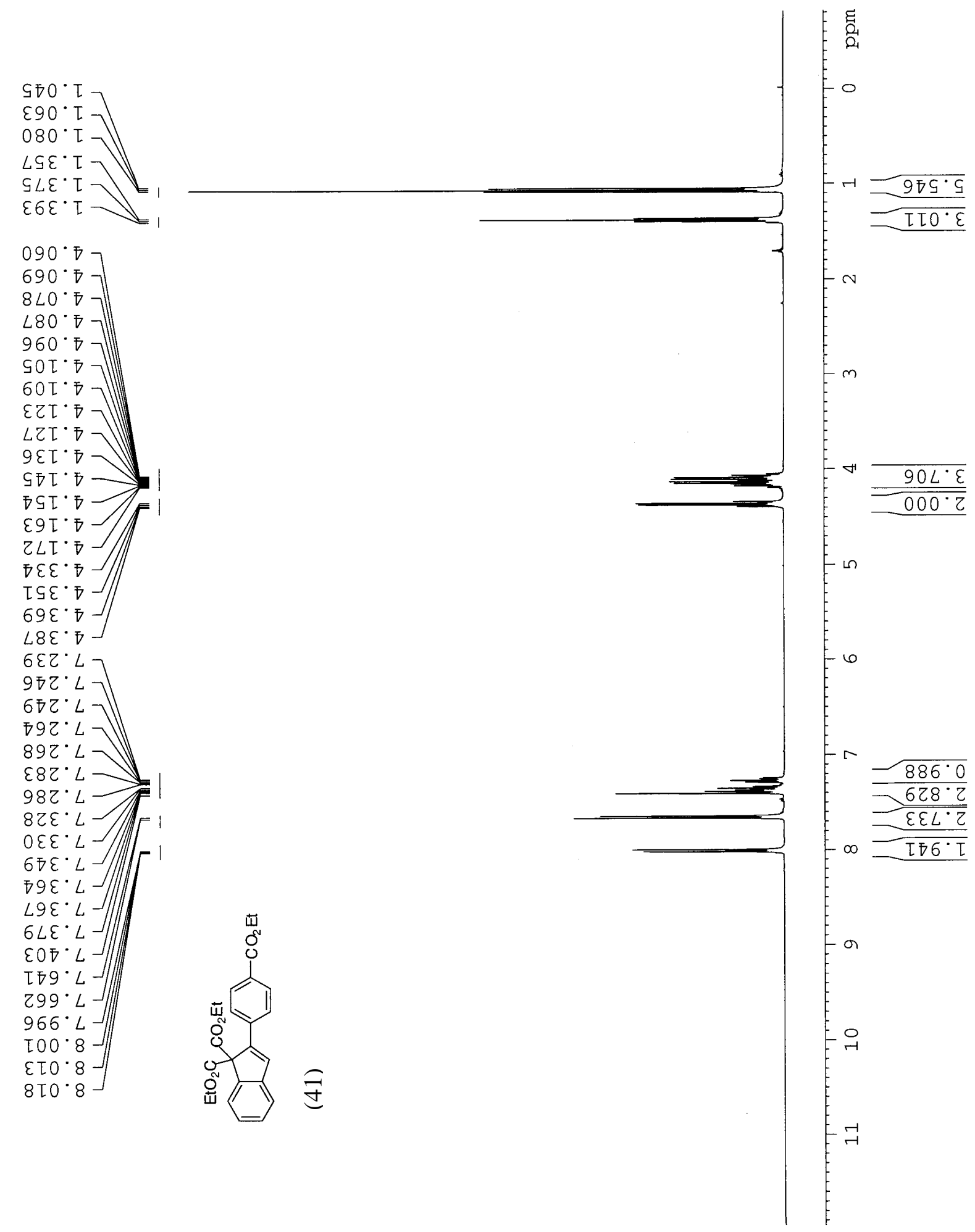


$8 乙 8 \cdot \varepsilon \tau \longrightarrow$

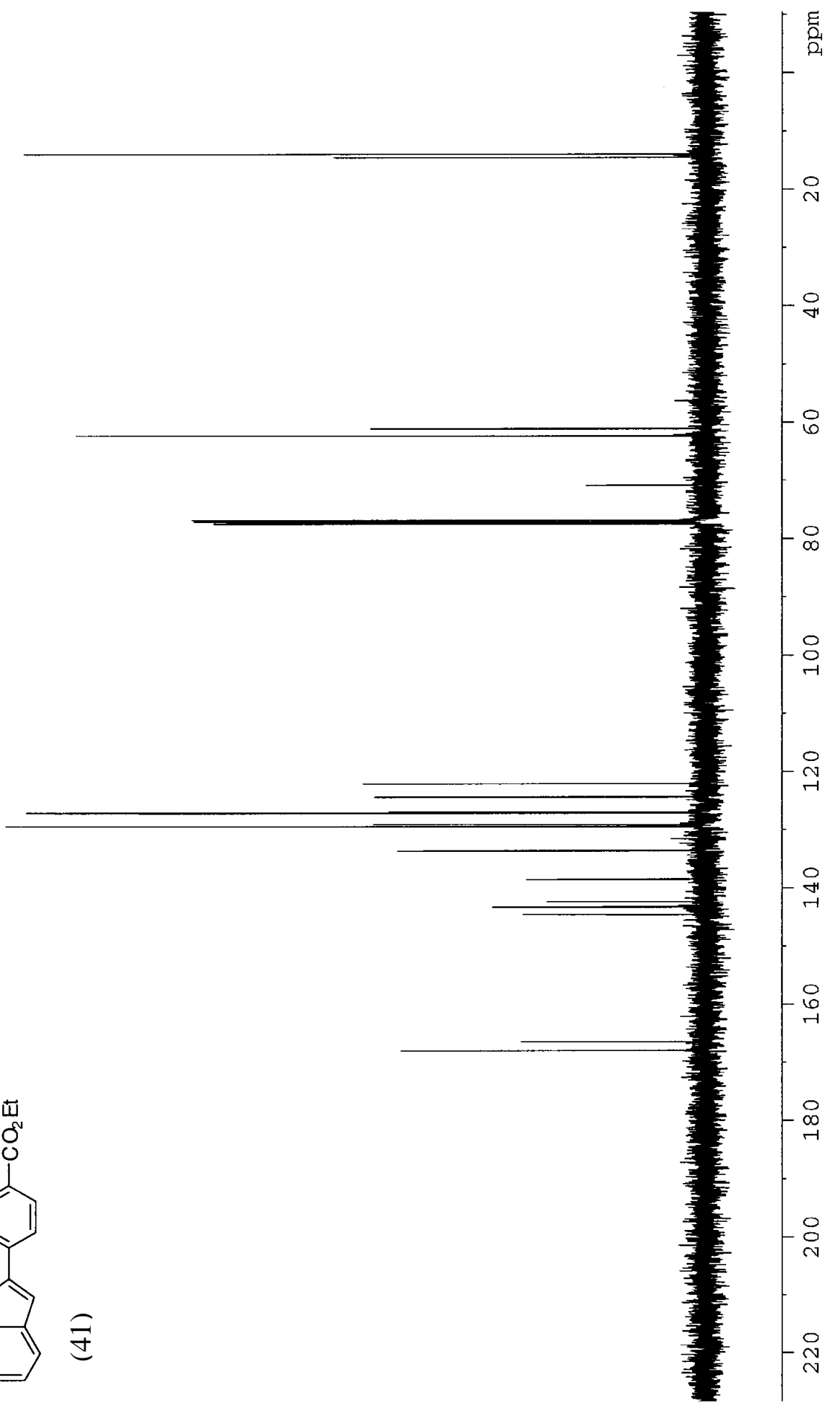

$690^{\circ} \mathrm{zz}$

ธย์

ऽ $6^{\circ} 9 Z \mathrm{Z}$

$20 T^{\circ} \angle Z T$

$\overline{750} 0^{\circ} 6 Z$

$\varepsilon \tau \nabla \cdot 6 Z \tau$

6๑ฑ $6 Z \tau$

$699^{\circ} \varepsilon \varepsilon$

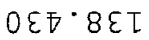

ॠ须

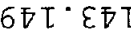

ฤらあ・市

$\angle 0 D^{\circ} 99 \mathrm{~T}$

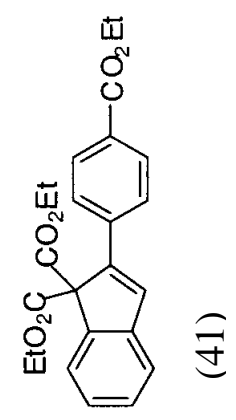

$\underset{-1}{\infty}$

$\stackrel{\circ}{\circ}$

옥 


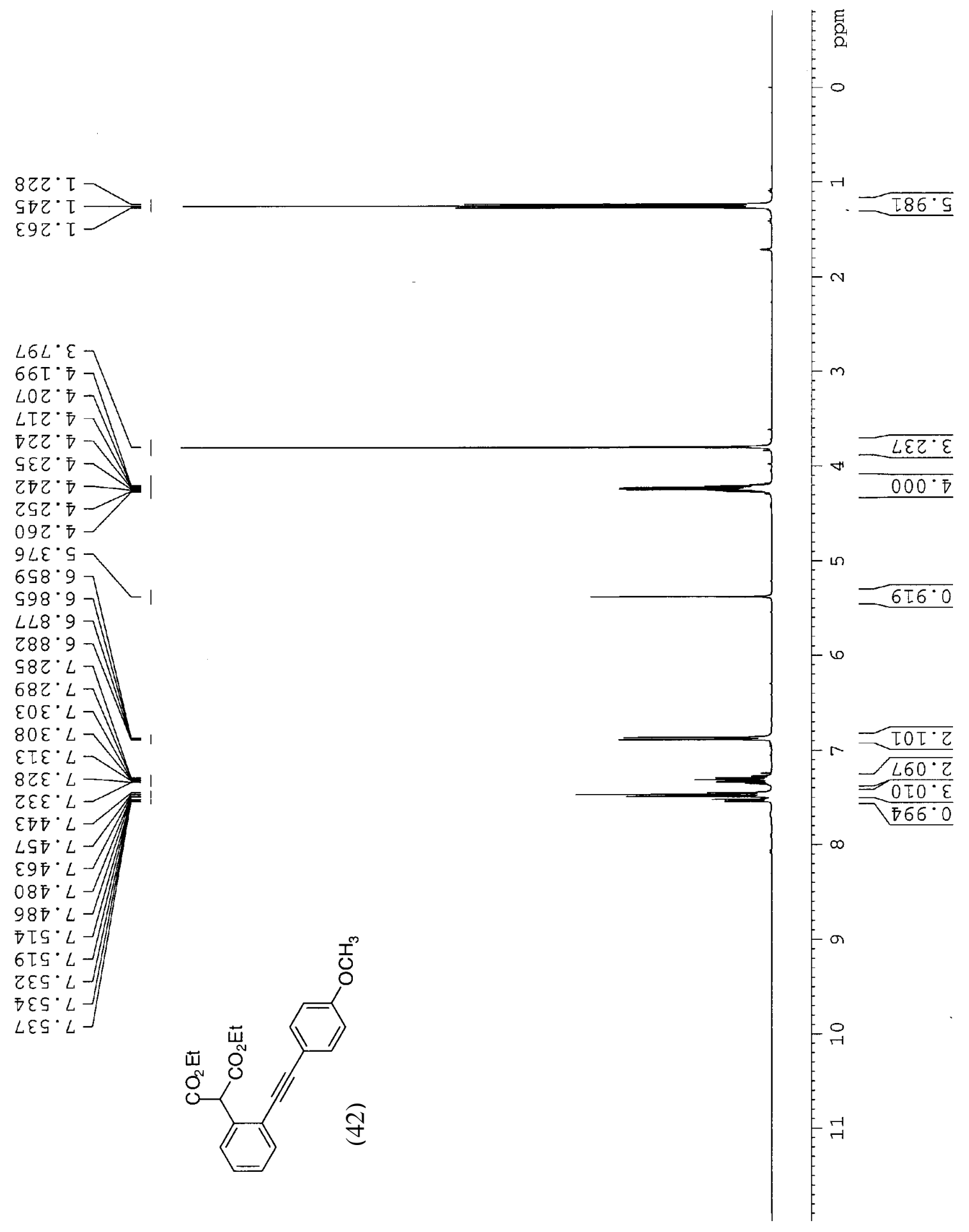


GOL' $\nabla I$

$09 \varepsilon \cdot \varsigma \varsigma$

OST.9S

$806^{\circ}$ โ9

$758 \cdot 9 L$

TLT' $\angle L$

$067^{\circ} L L$

$769^{\circ} 98$

9டL・テ6

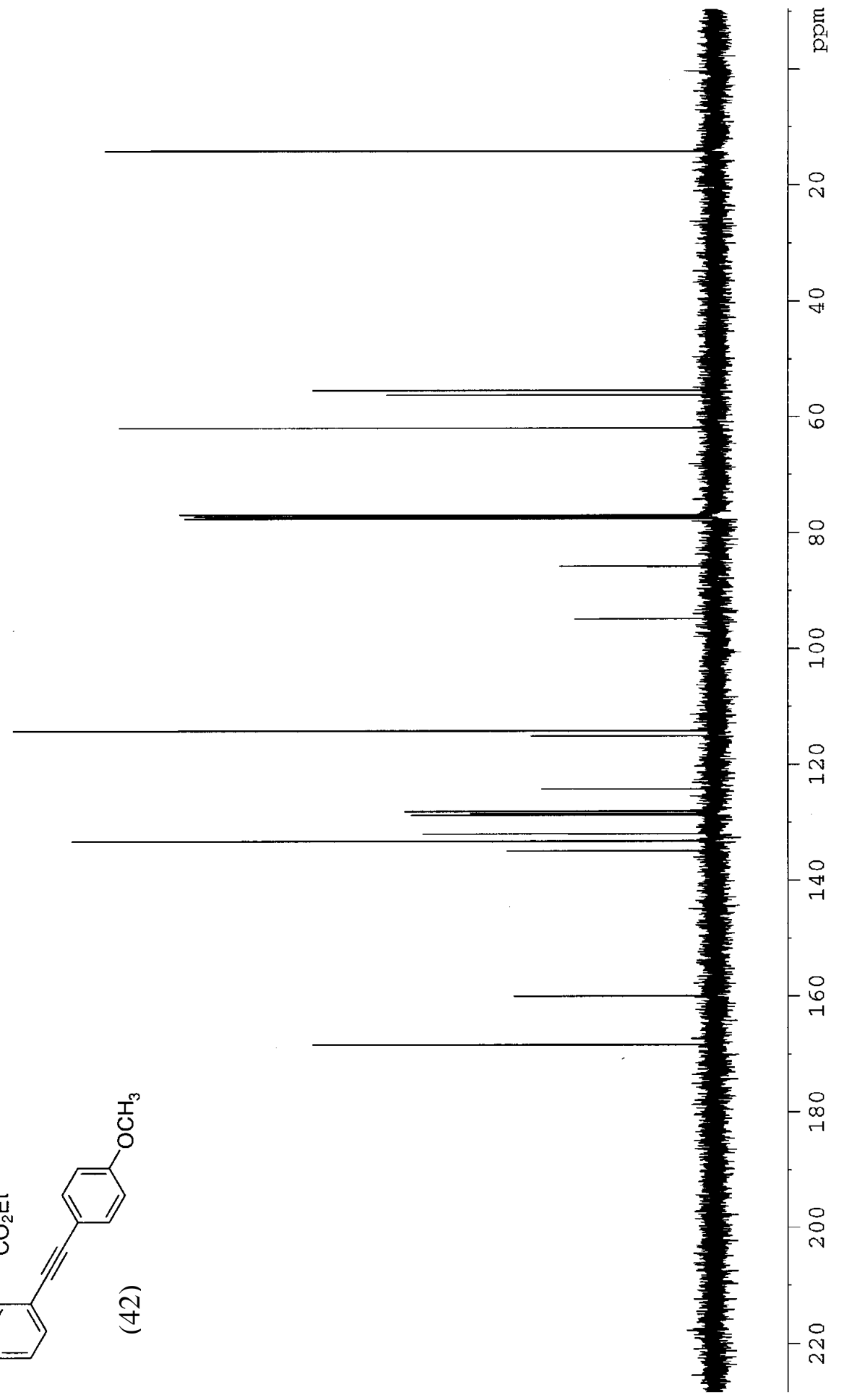

苈

$60 \tau \cdot 7 \mathrm{TL}$

SIO.SIT

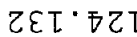

$686^{\circ} \mathrm{LZT}$

998.8ZT

56 $.82 \mathrm{I}$

$898^{\circ}$ T $\mathrm{T}$

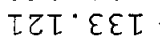

$69 L^{\circ}$ ๑ $\mathrm{L}$

$8 \nabla 6^{\circ} 6 \mathrm{~T} \mathrm{~T}$

$9 L Z \cdot 89 I$ 


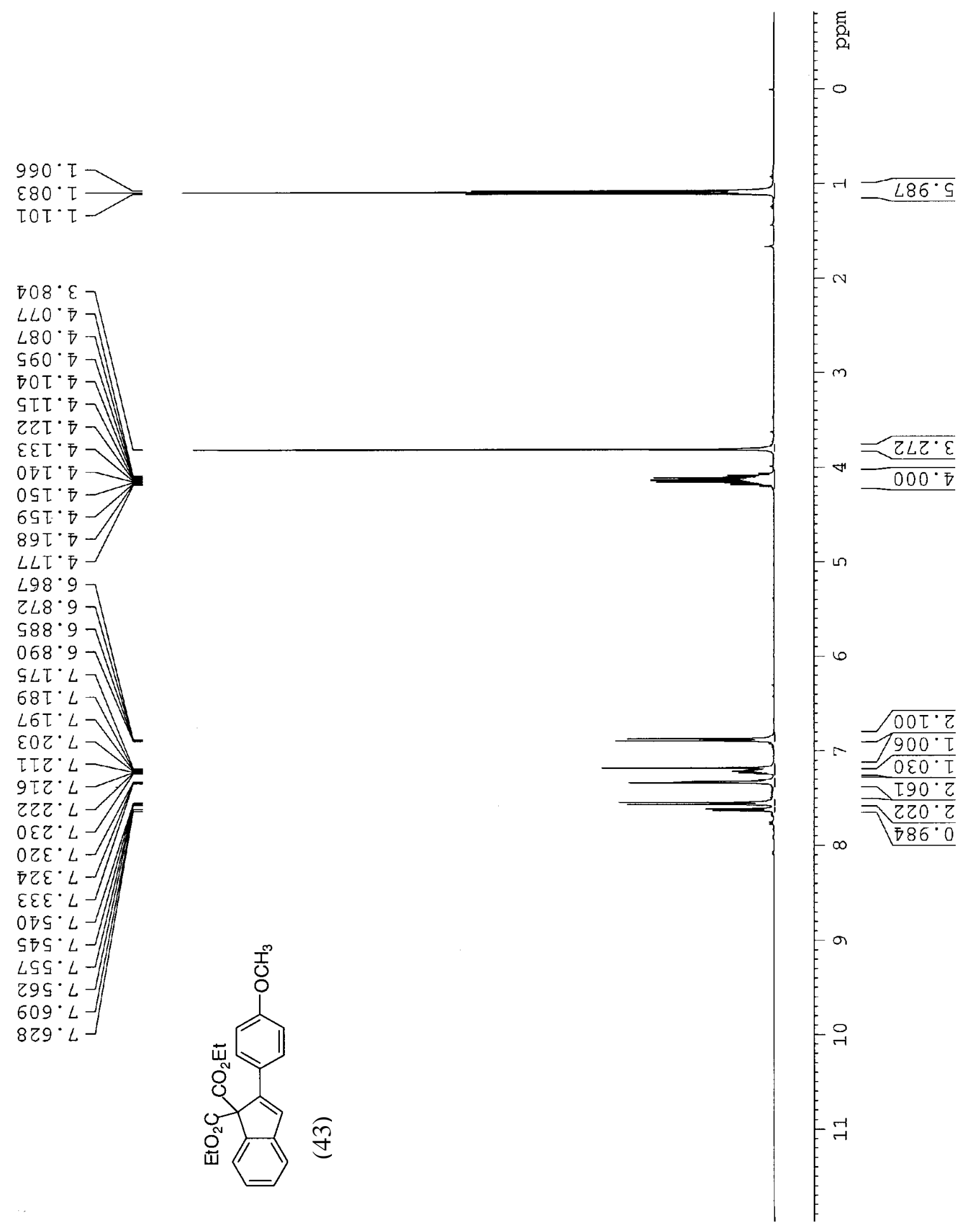


โIย. ऽ

$920 \cdot 29$

$568.0 L$

$9 \nabla 8 \cdot 9 L$

$\varepsilon 9 \tau \cdot L L \longrightarrow$

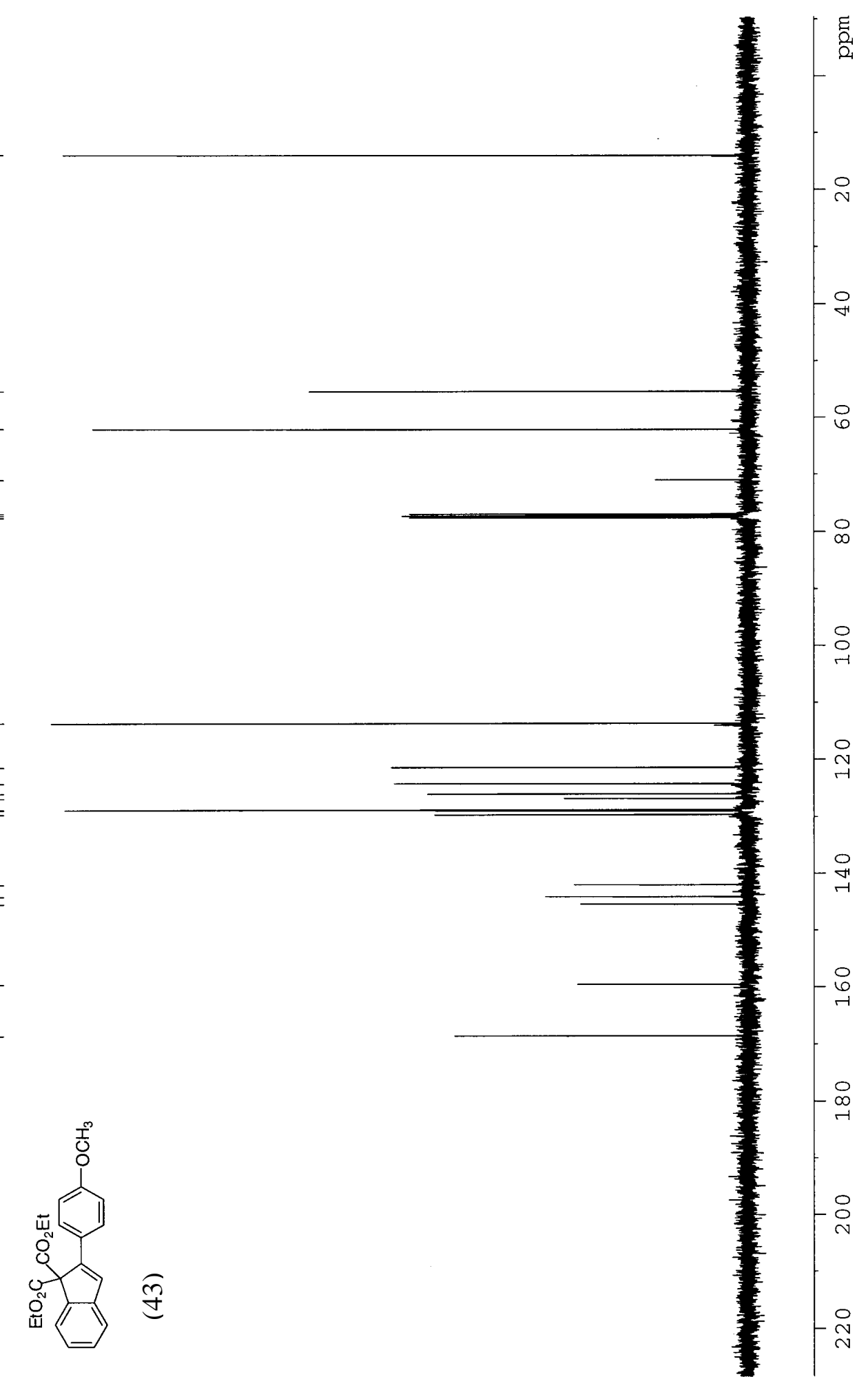

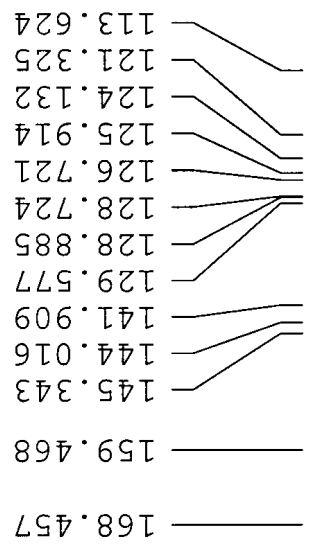




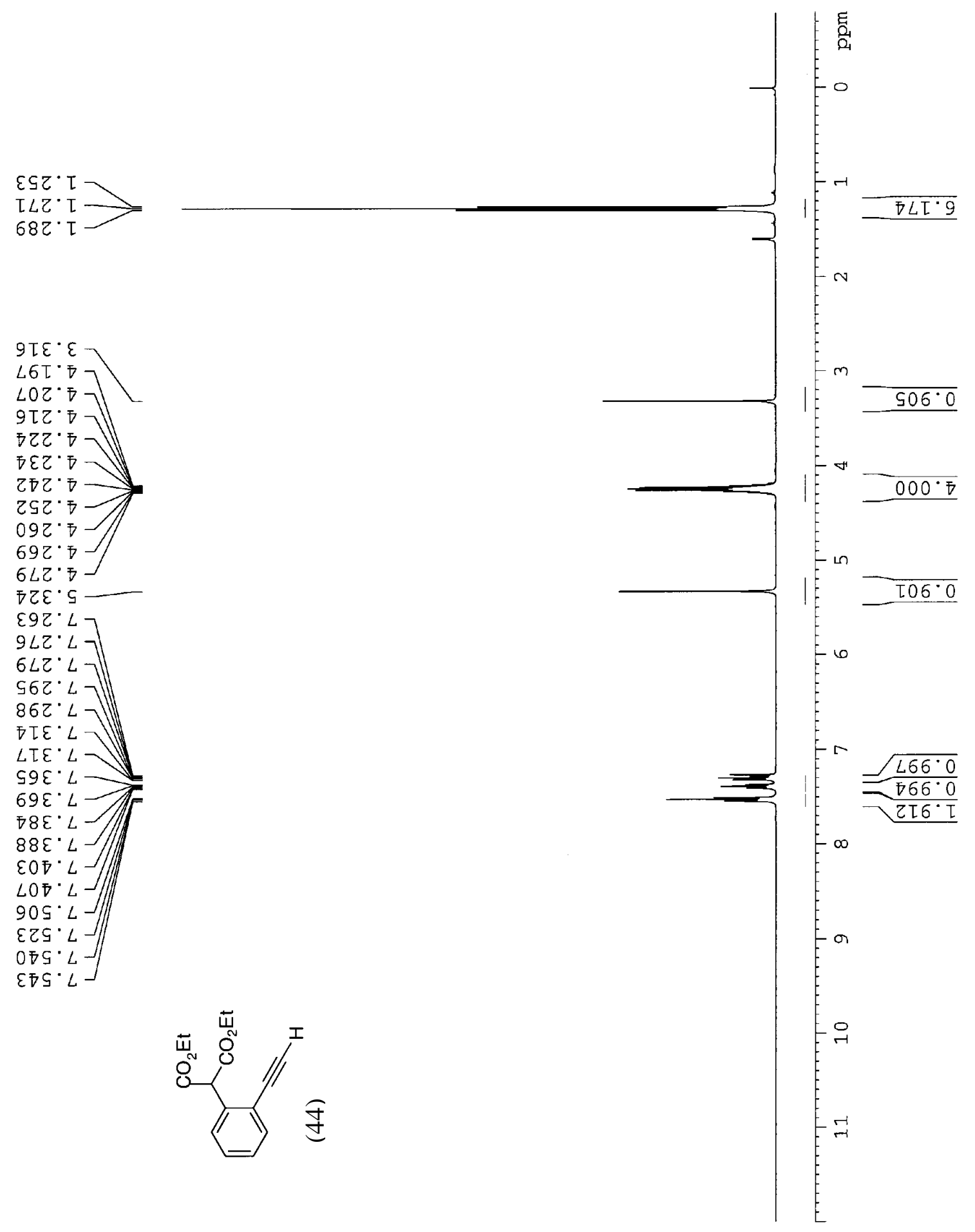


$8 \angle 0^{\circ} D T$

LES.SS

9 $\sigma^{*}$ โ9

$8 S L \cdot 9 L$

$S L 0^{\circ} \angle L$

$\varepsilon 6 \varepsilon^{\circ} L L$

$08 \mathrm{~L} \cdot \mathrm{L8}$

ILZ'Z8

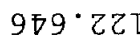

E86 $\mathrm{LZT}$

T6L.8ZT

$98 T \cdot 6 \tau T$

$\angle 08^{\circ}$ 乙ع

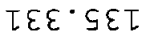

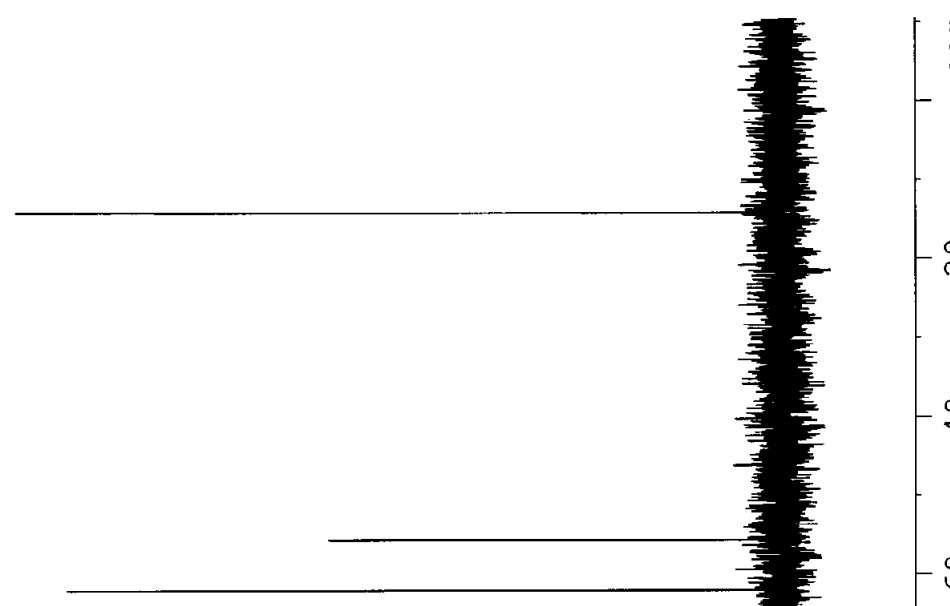

है

오

욱

웅

$-\infty$

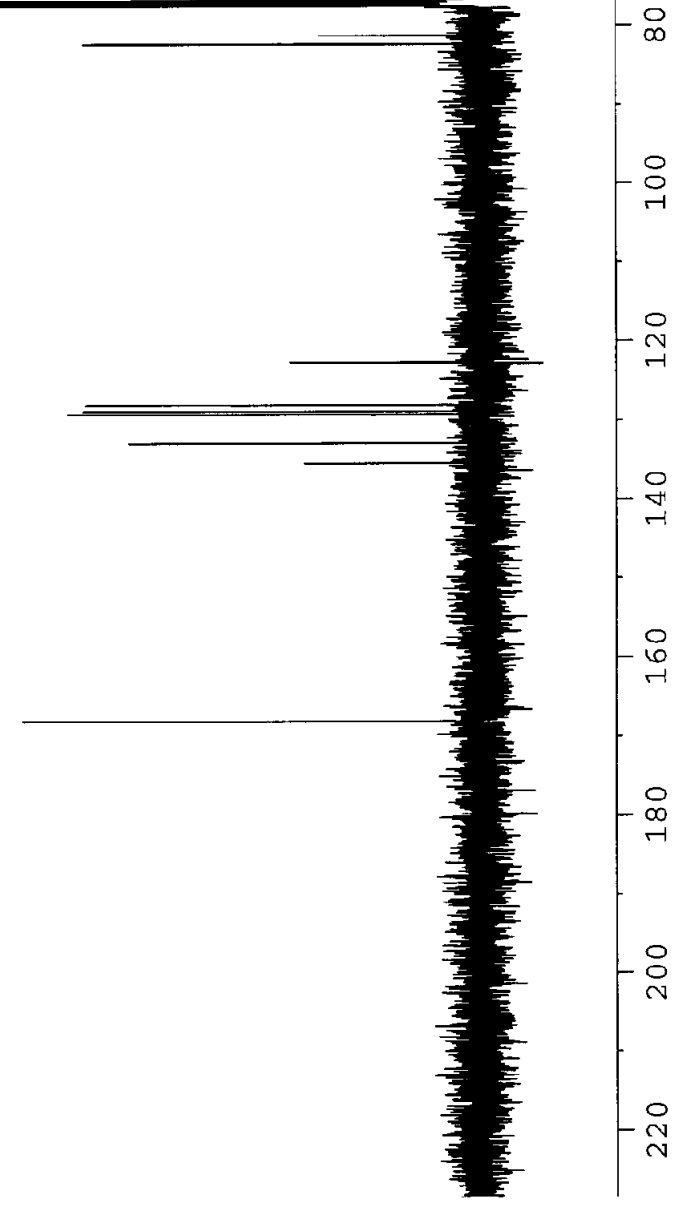

$-8$

$\rightarrow$

$\stackrel{\circ}{\rightarrow}$

$600.89 I$

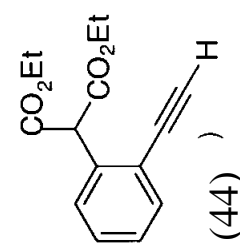




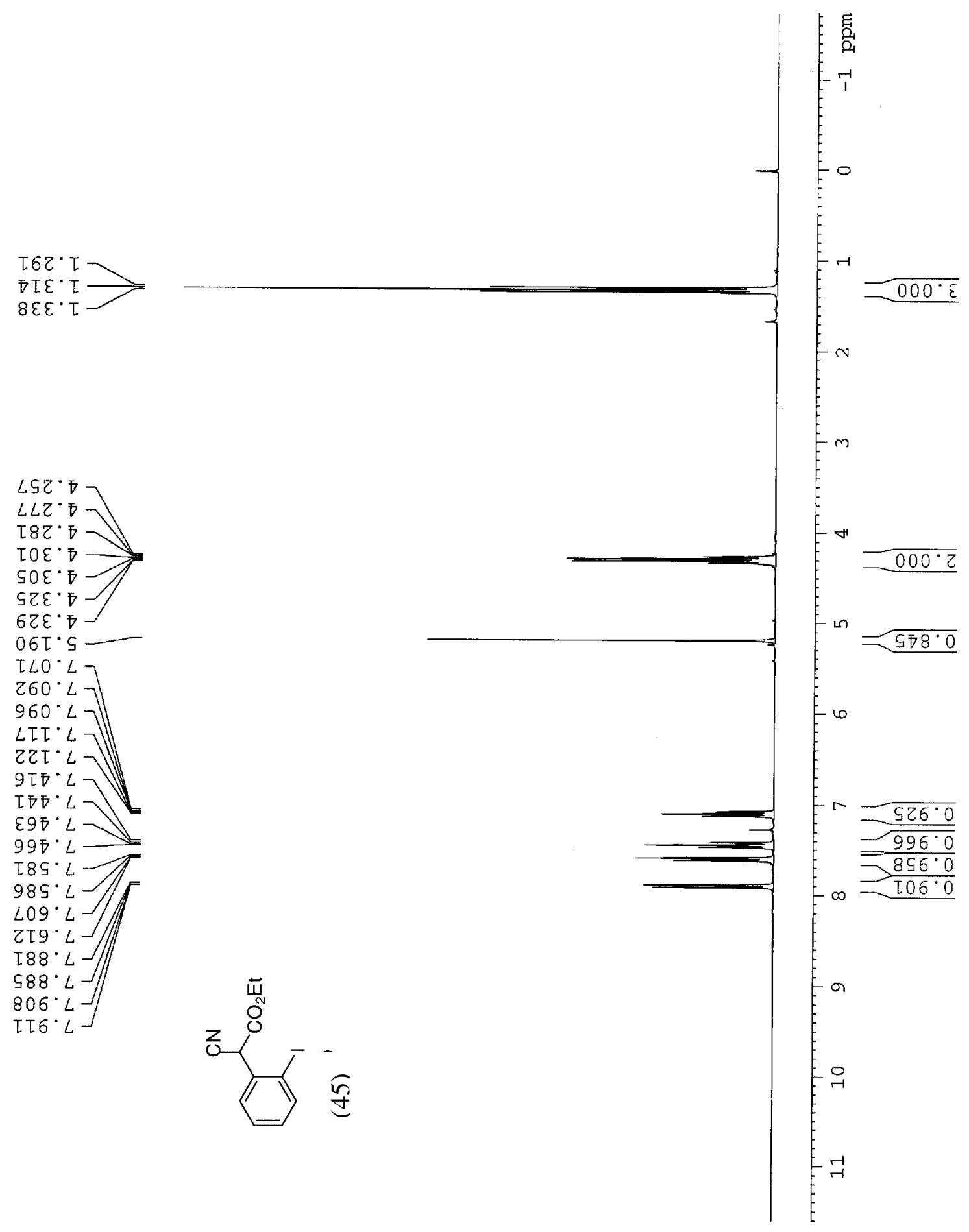


$28 \mathrm{~T} \cdot \tau \mathrm{I}$

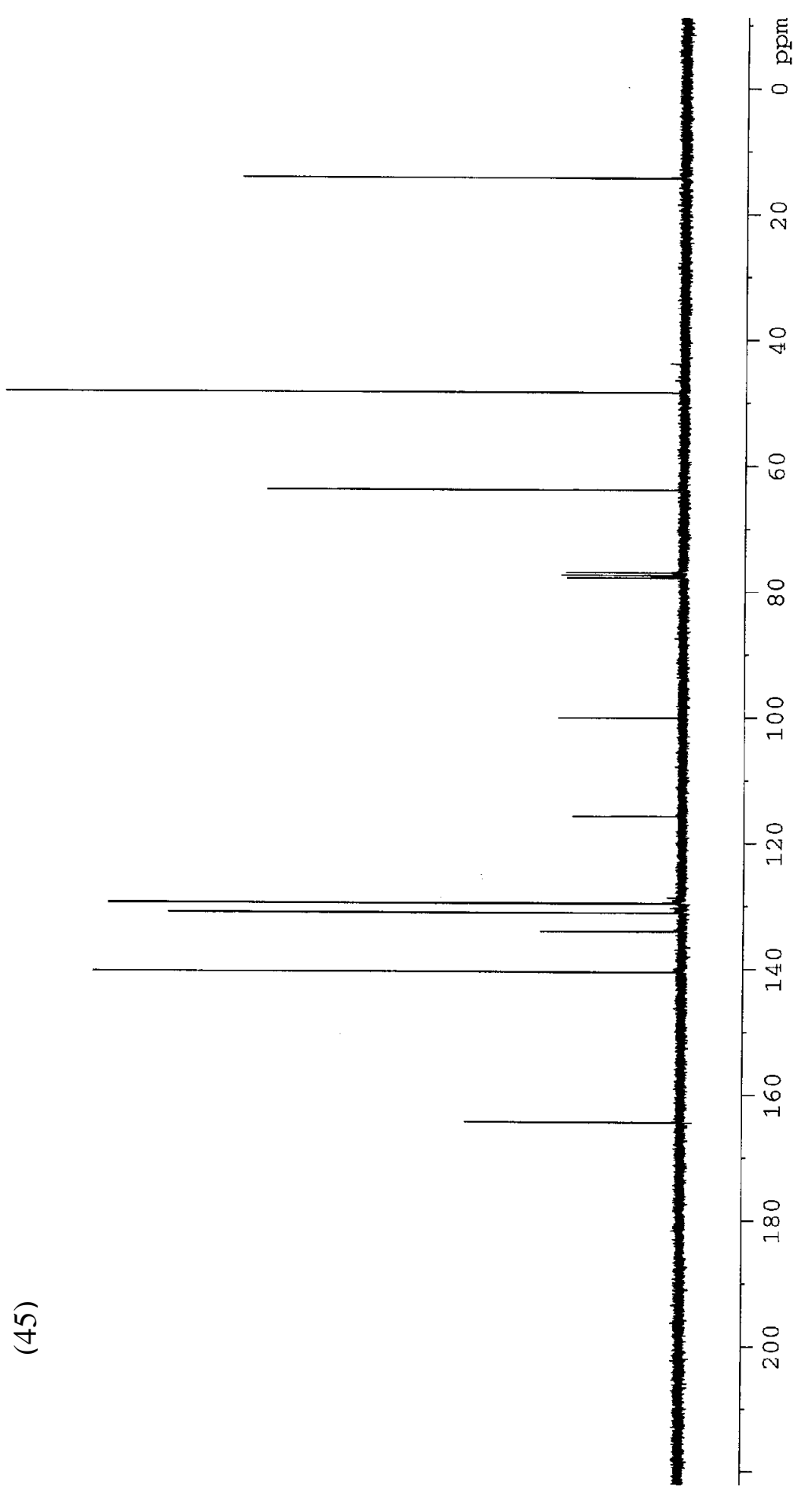

ธฤย・ซ9โ

茪 


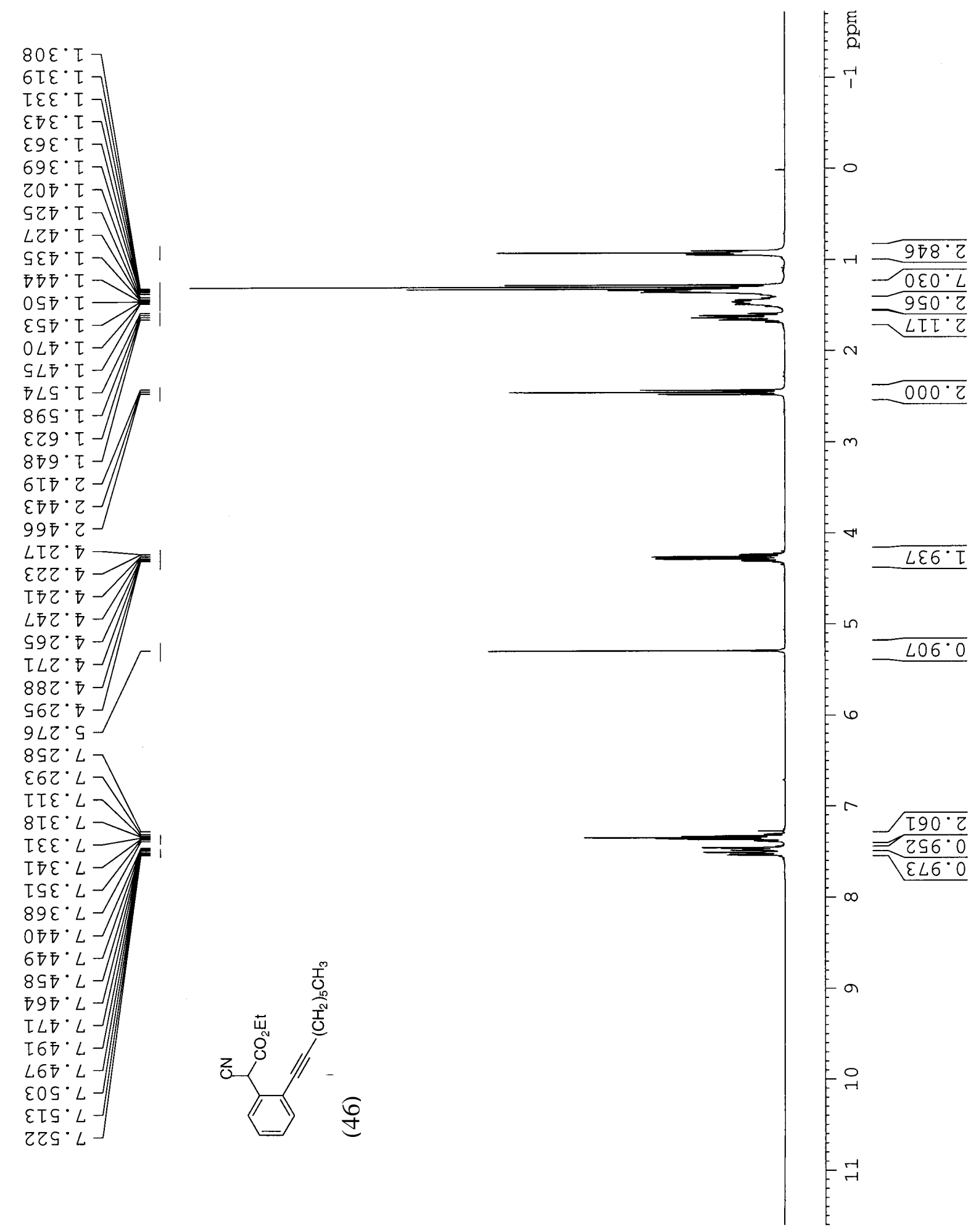




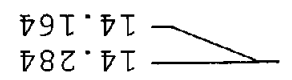

$9 S L \cdot 6 \tau$

ธLL ZZ

62L.82

$568 \cdot 87$

हร与 $T E$

$820^{\circ}$ 乙万

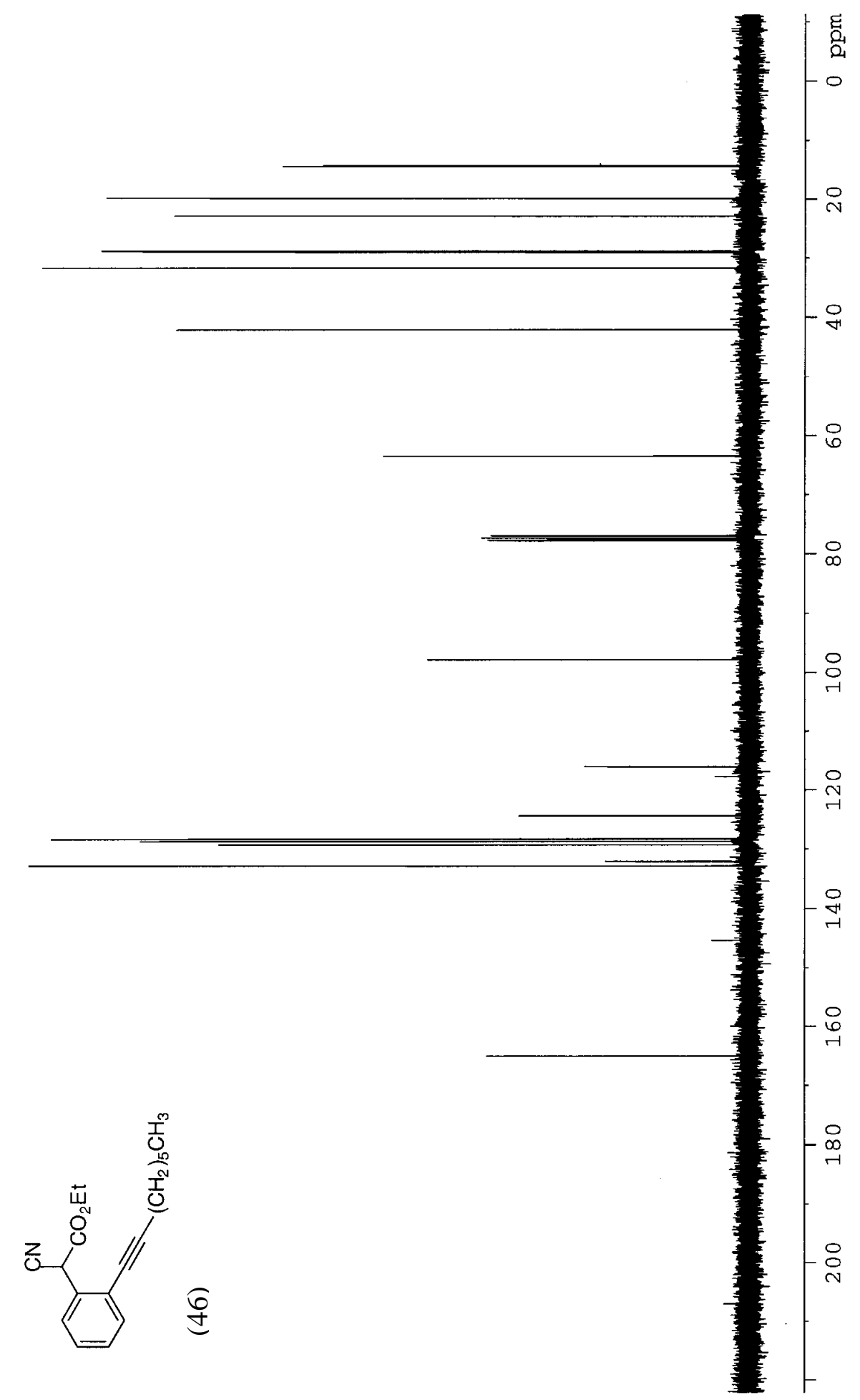

$896^{\circ}$ Ð 9

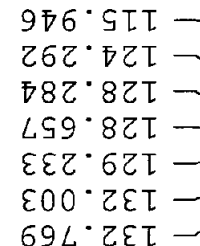

$\nabla 98 \cdot 9 L$

$\angle 82^{\circ} \angle L$

$E O S^{\circ} L L$
$\tau T L \cdot L L$

$\varepsilon 8 L \cdot L 6$

$69 L^{\circ}$ ZET

$896^{\circ} \nabla 9$

$\stackrel{\infty}{-}$

$\stackrel{8}{8}$ 


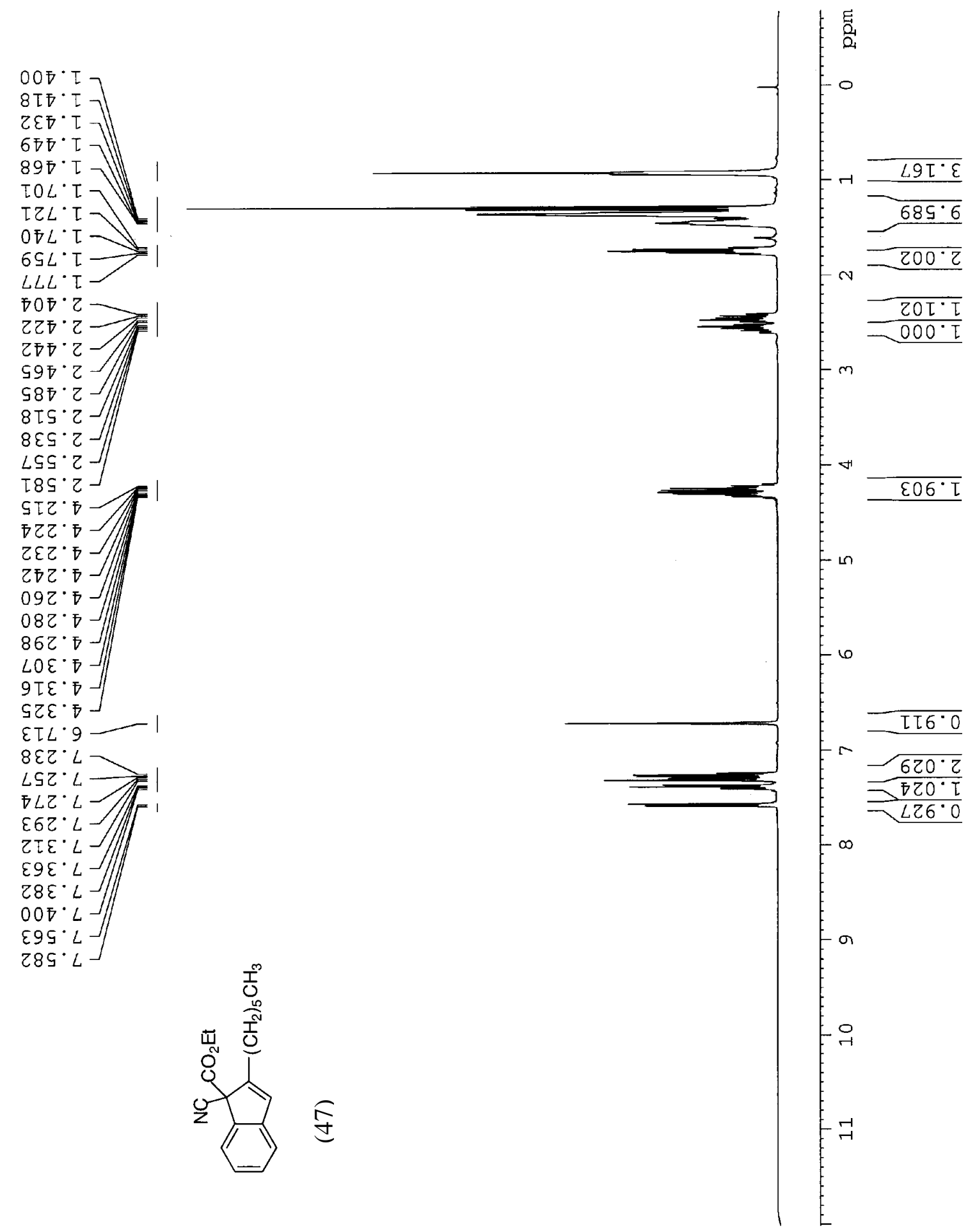



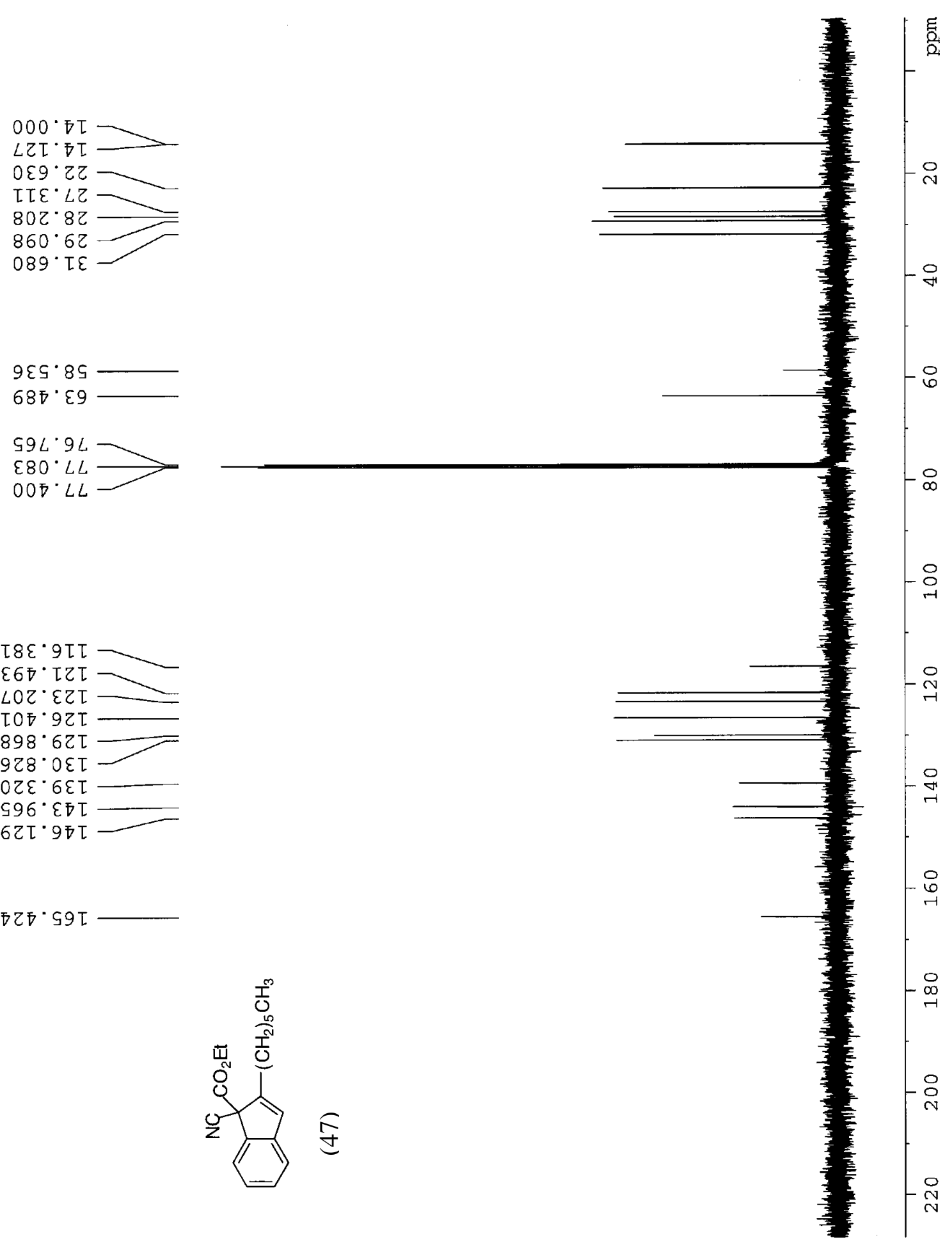

$\tau 8 \varepsilon^{\circ} 9 \tau \tau-$

$\varepsilon 6 \varpi^{\circ} \tau Z \tau$

$\angle 0 Z \cdot \varepsilon Z T$

IOT.9ZT

(2008.

9Z8. $0 \varepsilon T$

$0 Z \varepsilon^{\circ} 6 \varepsilon \tau$

$\varsigma 96^{\circ} \varepsilon \bar{\tau} \tau$

$6 乙 \tau \cdot 9 \succsim \tau$

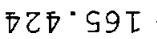




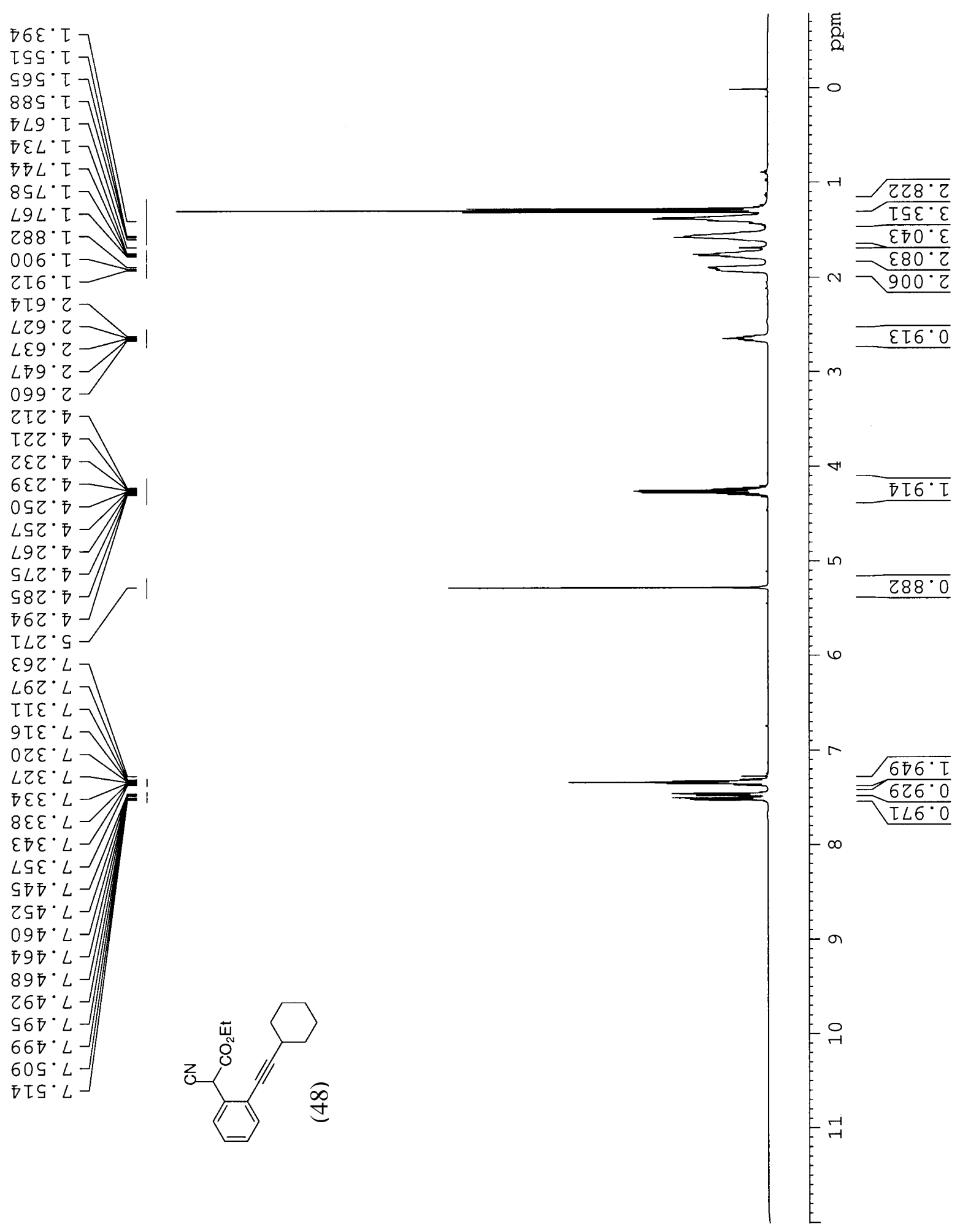




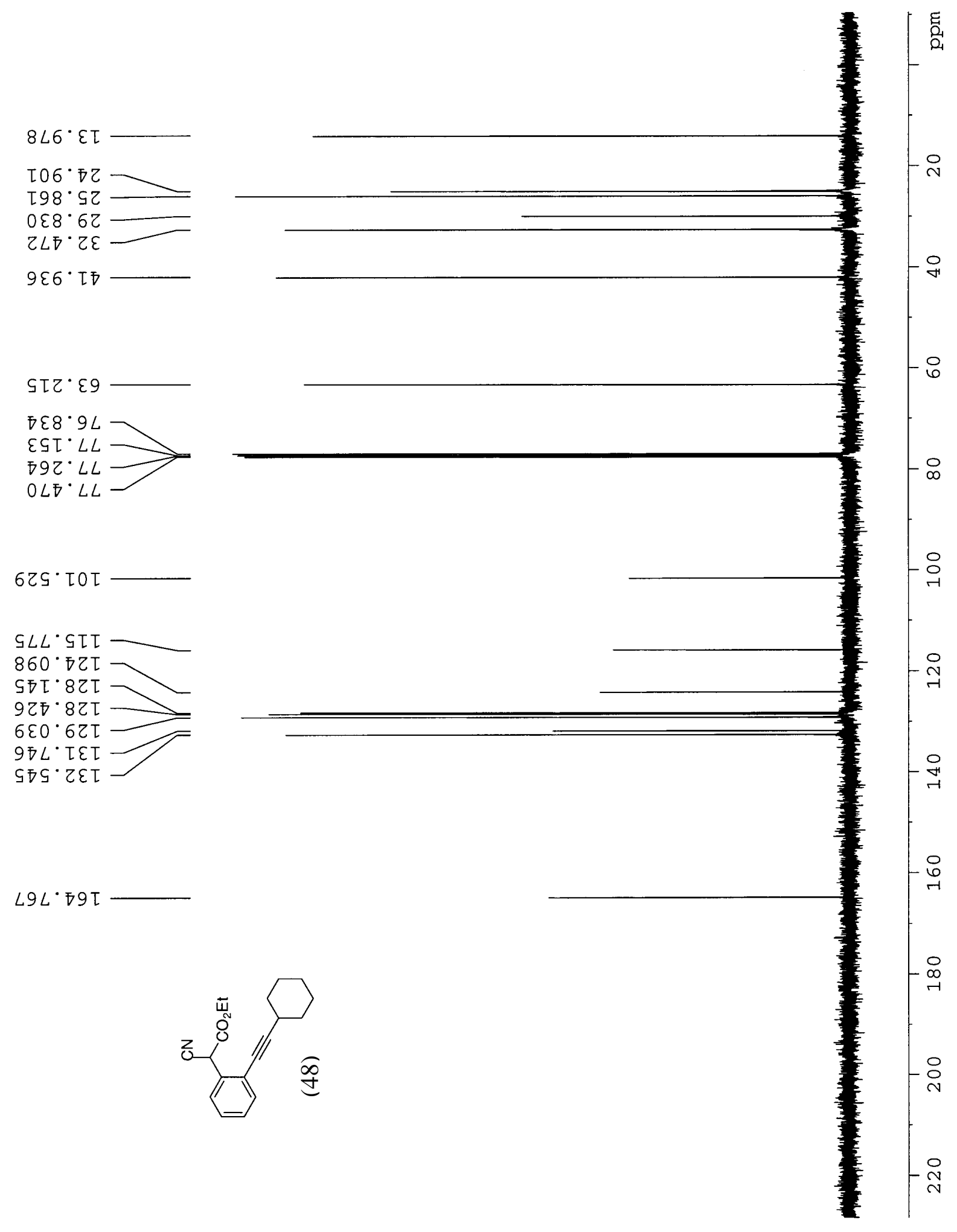



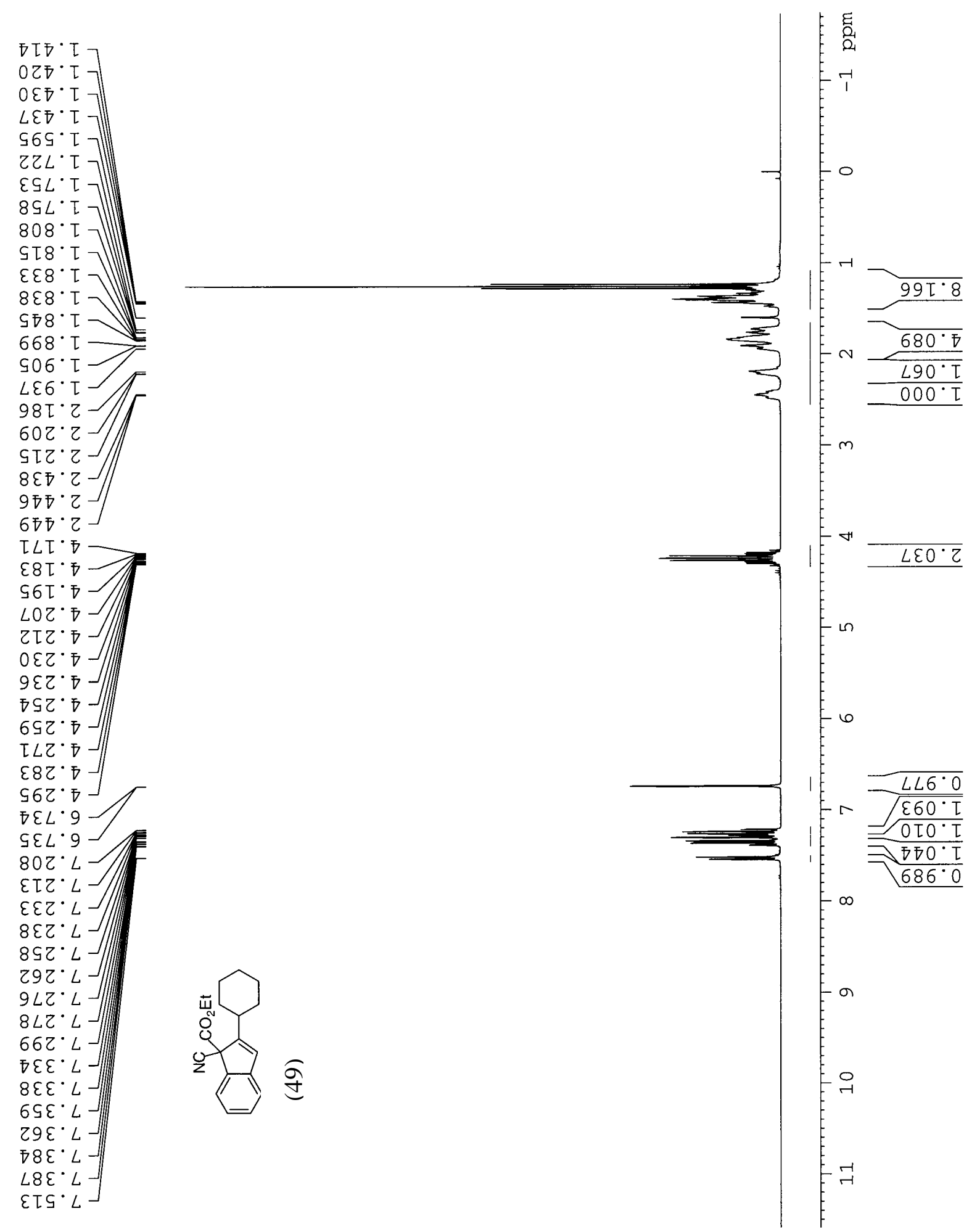


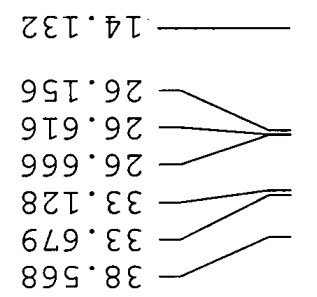

$6 \varepsilon 2 \cdot 89$

$889^{\circ} \varepsilon 9$
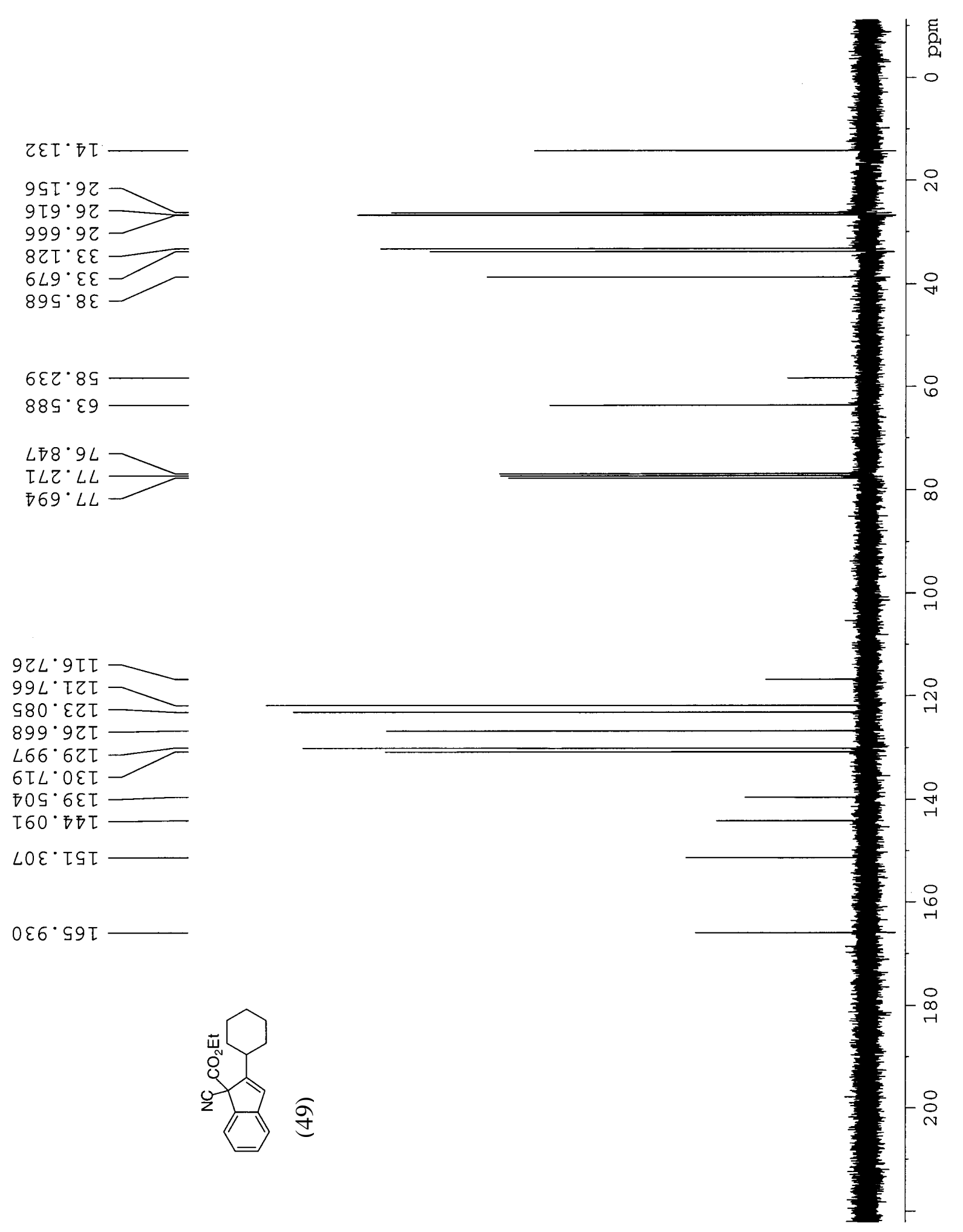


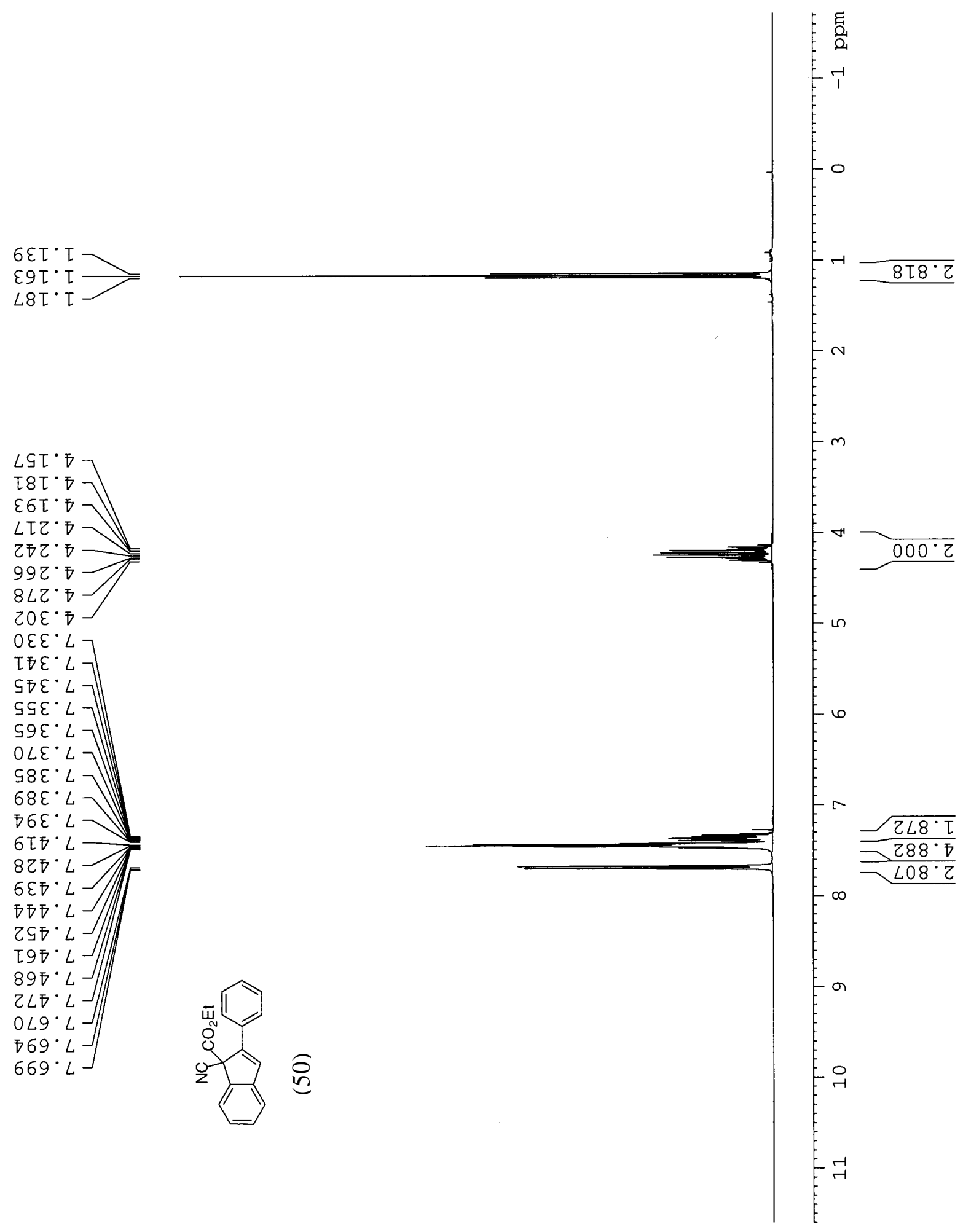


$\varsigma \mp 8 \cdot \varepsilon \tau$

$\angle \nabla L 9 S$

$969 \cdot \varepsilon 9$

$\overline{7} 88 \cdot 9 L$

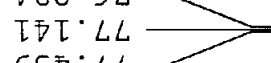

$655^{\circ} 4 L^{-}$
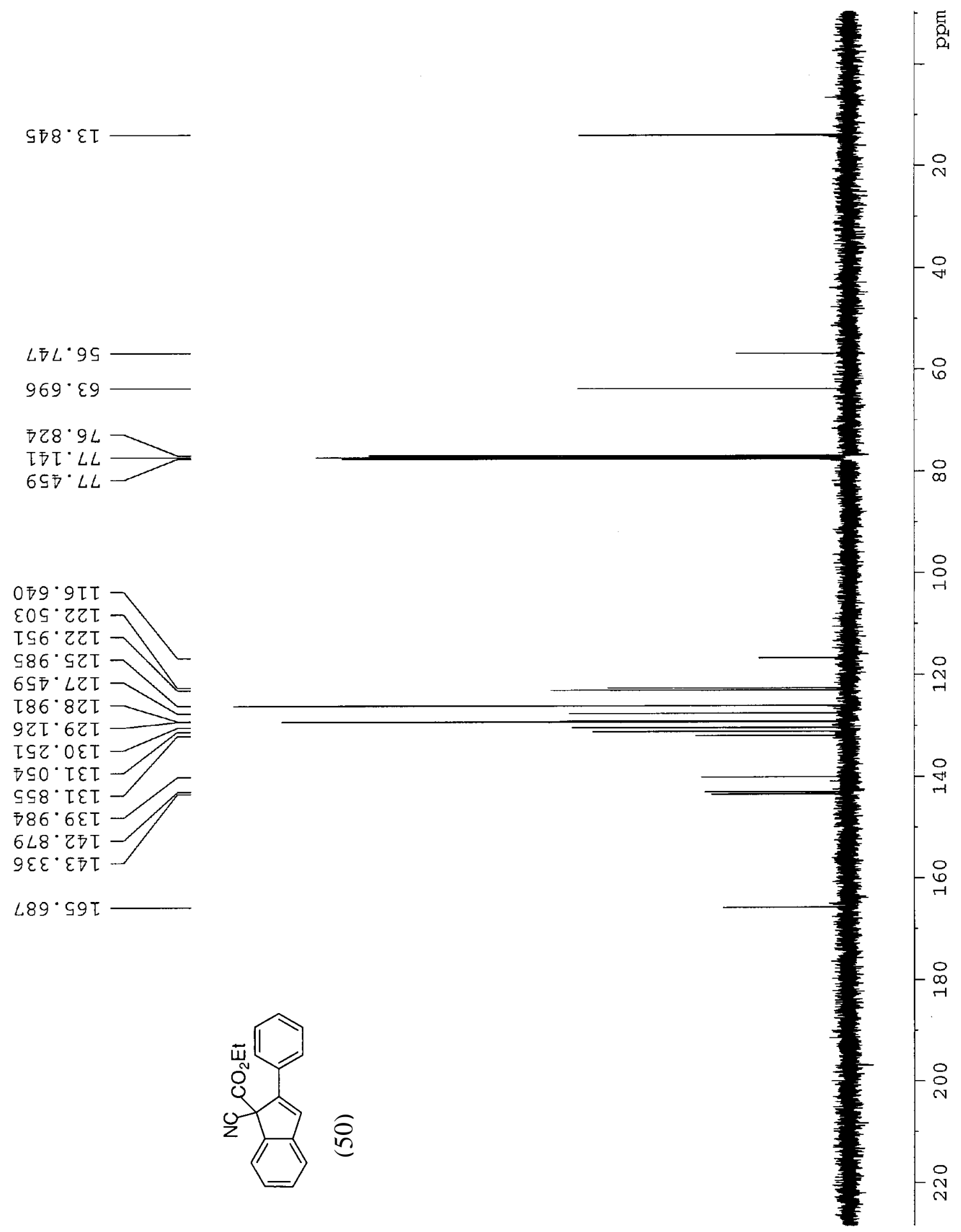


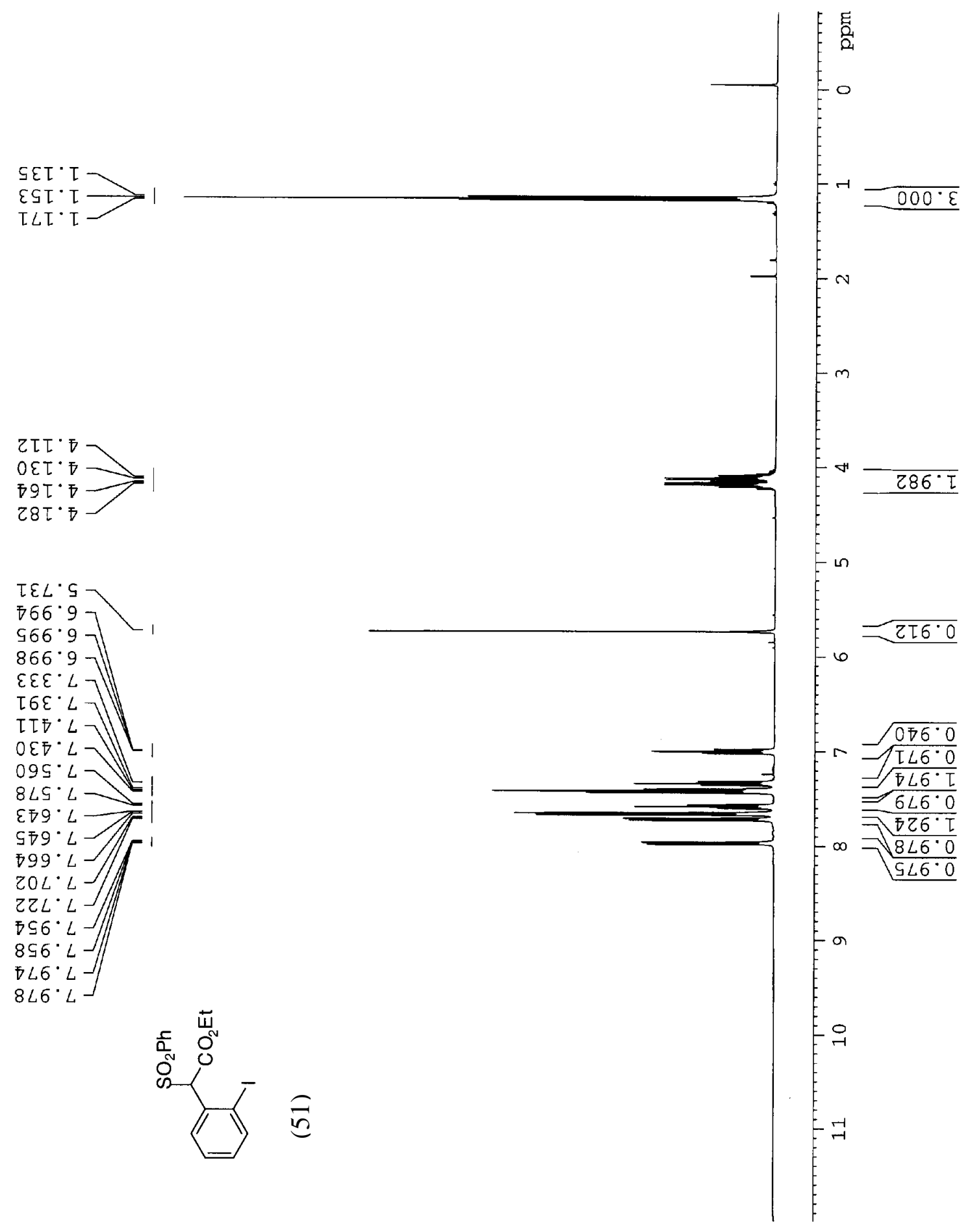




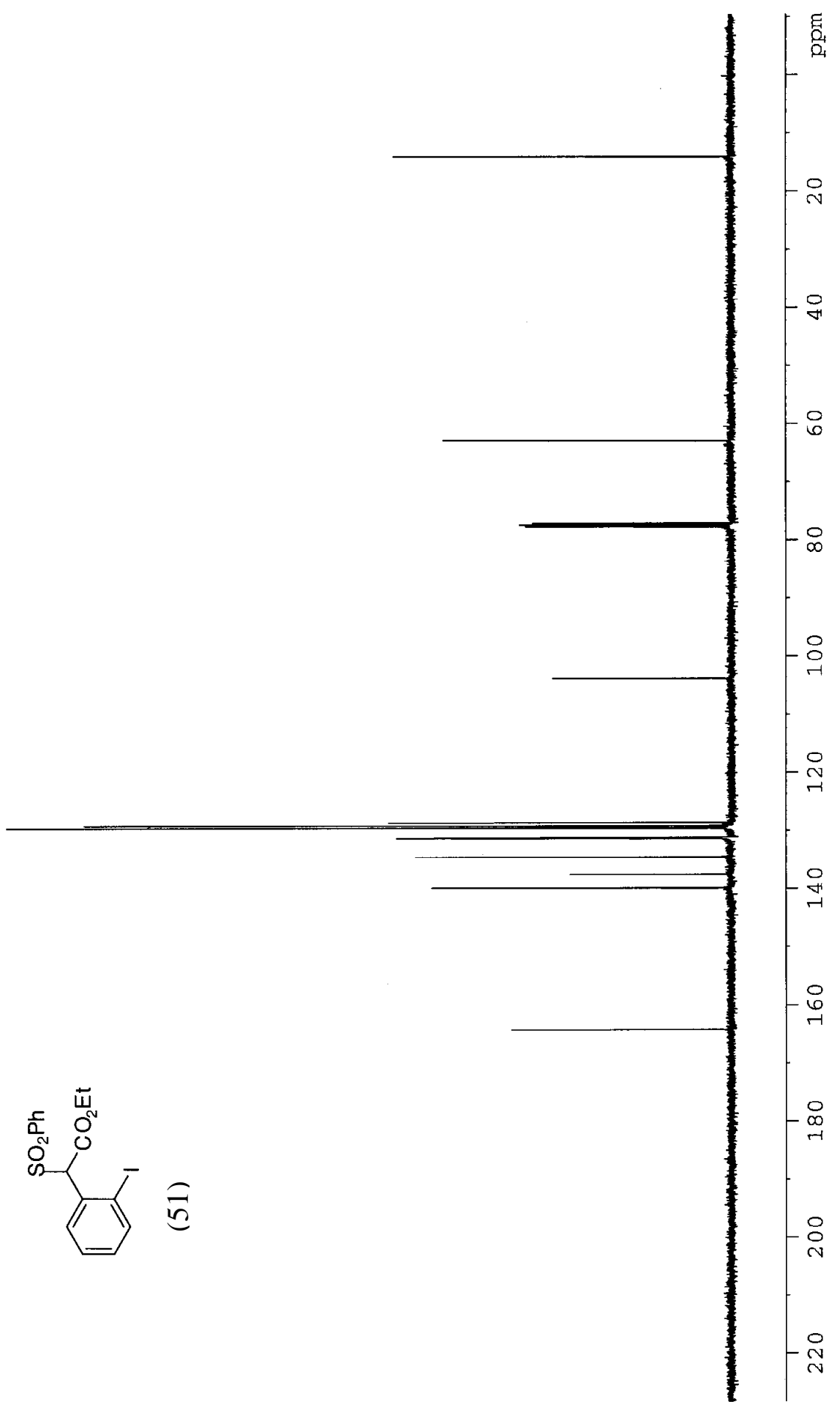

$620^{\circ} L L$ $\varepsilon \mp \varepsilon^{\circ} L L$

$999^{\circ} \angle L$

$\varepsilon \subseteq L \cdot \varepsilon 0 \tau$

T8G.8ZT
$00 \mathrm{I} \cdot 6 \mathrm{TI}$

Z6न $6 Z \mathrm{ZT}$

ฤ०โ โ $\varepsilon \tau$

$\nabla 6 \mathrm{I} \cdot \mathrm{L} \mathrm{\tau}$

$\nabla 6 \mathrm{Z}^{\circ} \tau \varepsilon \tau$

ธโธ. จยโ

Ђ9干 $\angle \varepsilon T$

$508^{\circ} 6 \varepsilon \tau$

$0 \mp \tau \cdot \nabla 9 \tau$

$\stackrel{\text { N }}{\sim}$ 


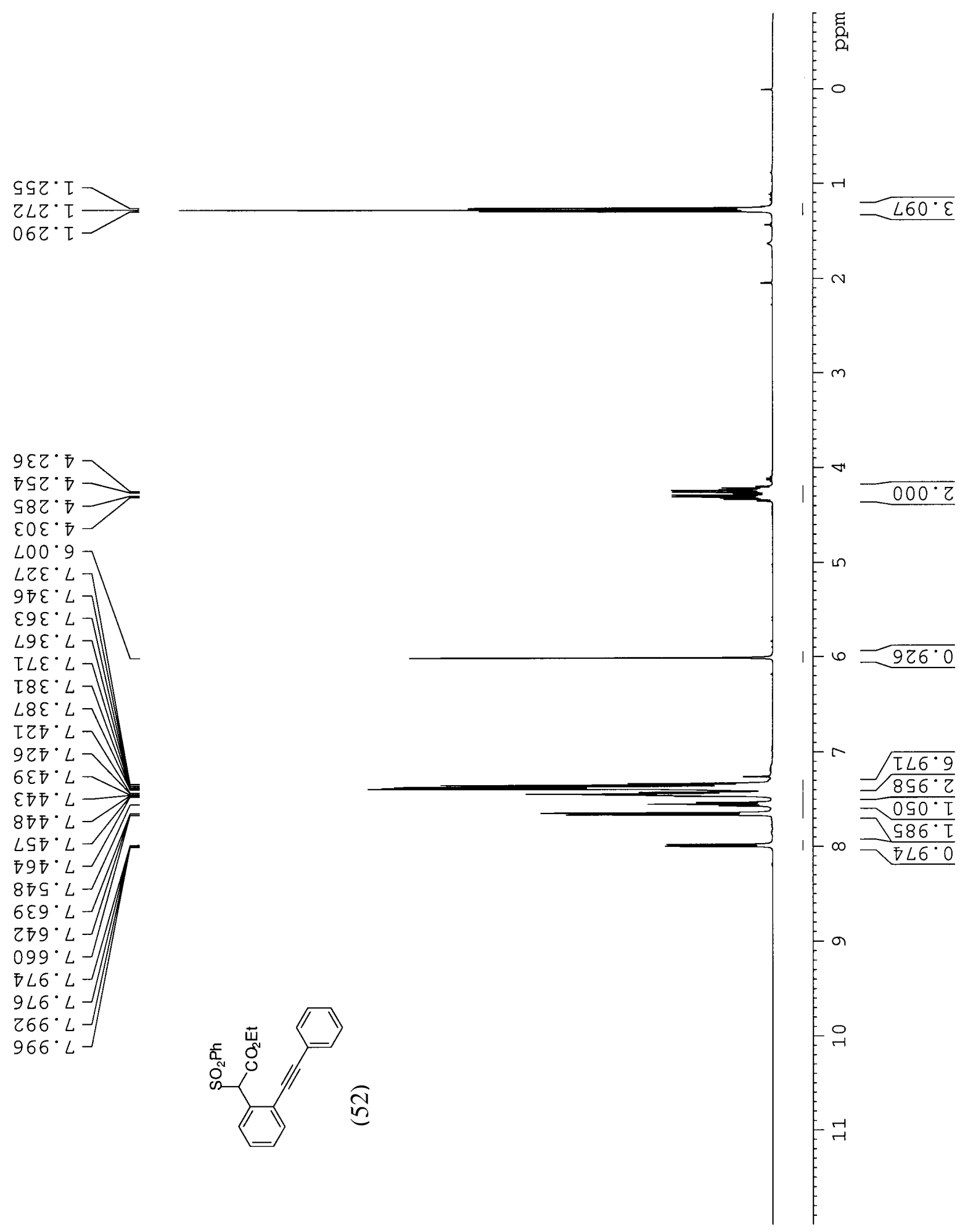



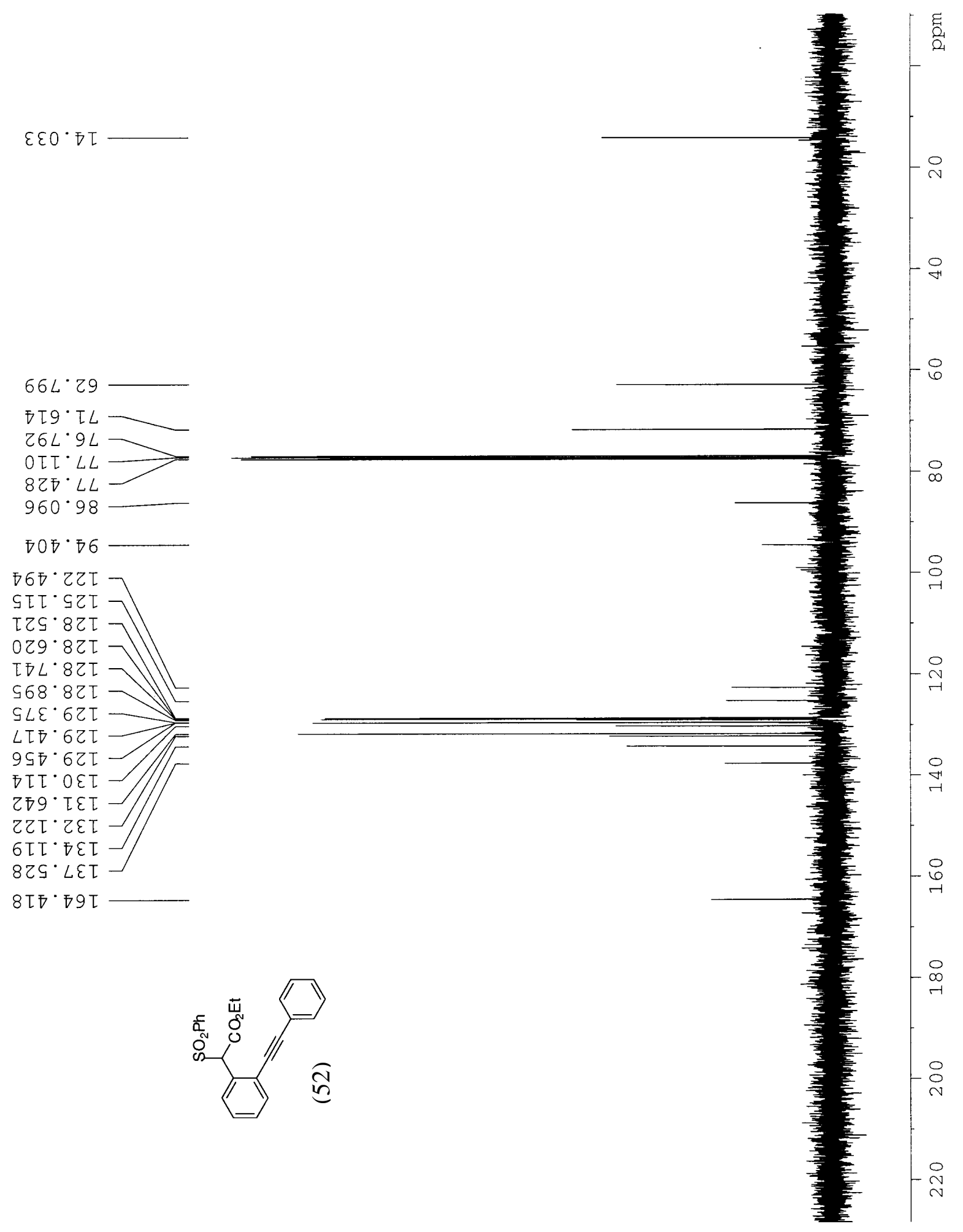

93S 


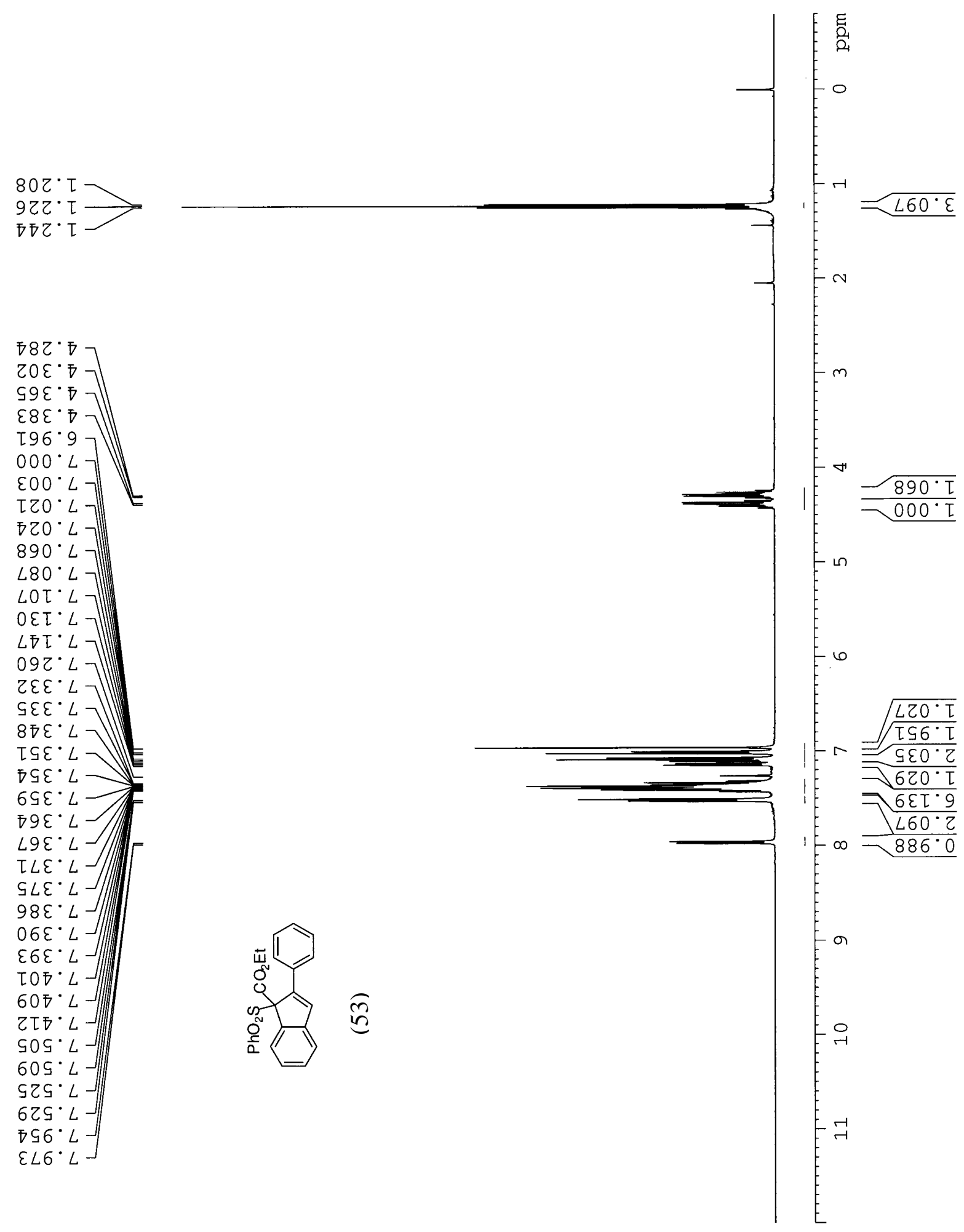


$9 โ \sigma^{\cdot} \varepsilon \tau$
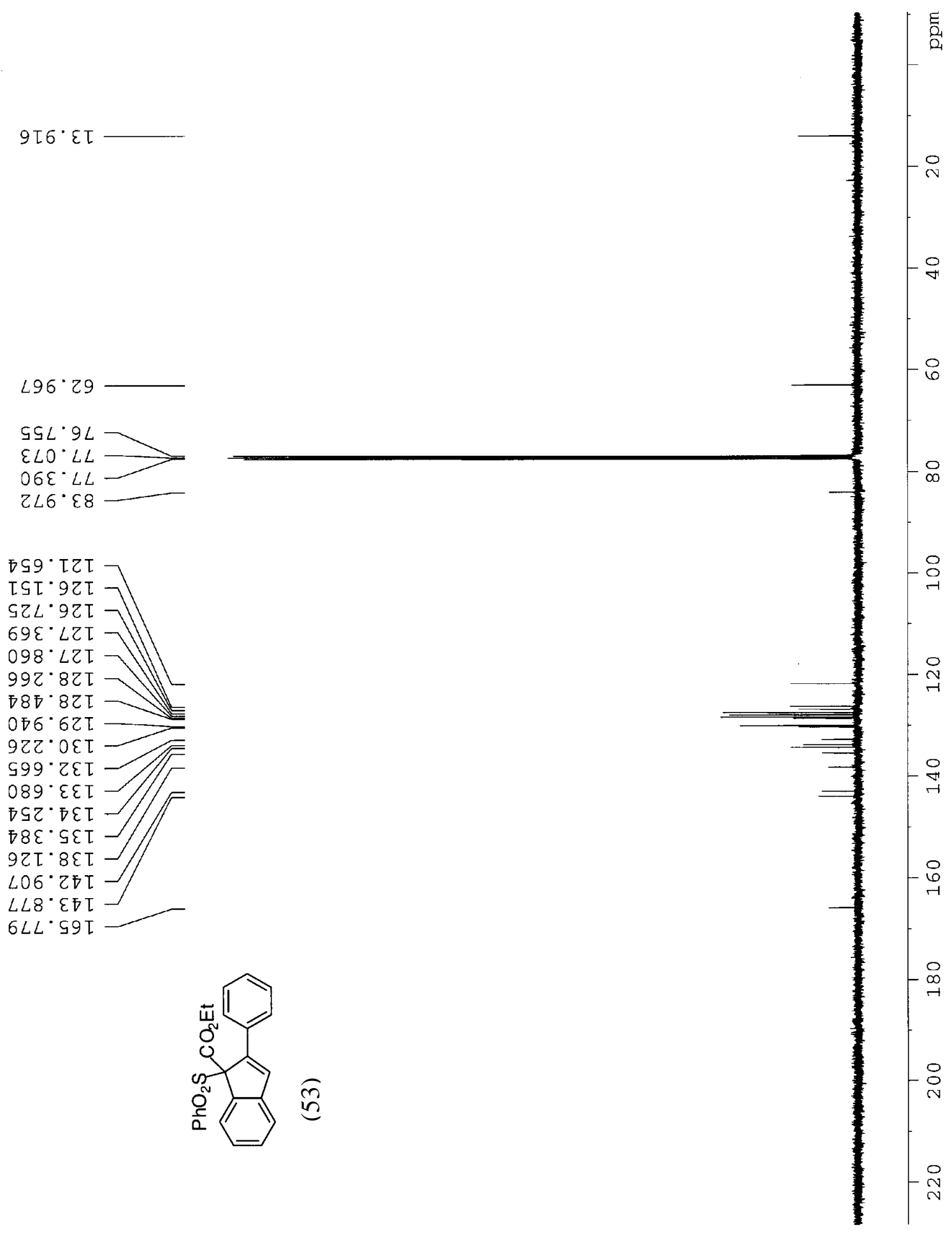

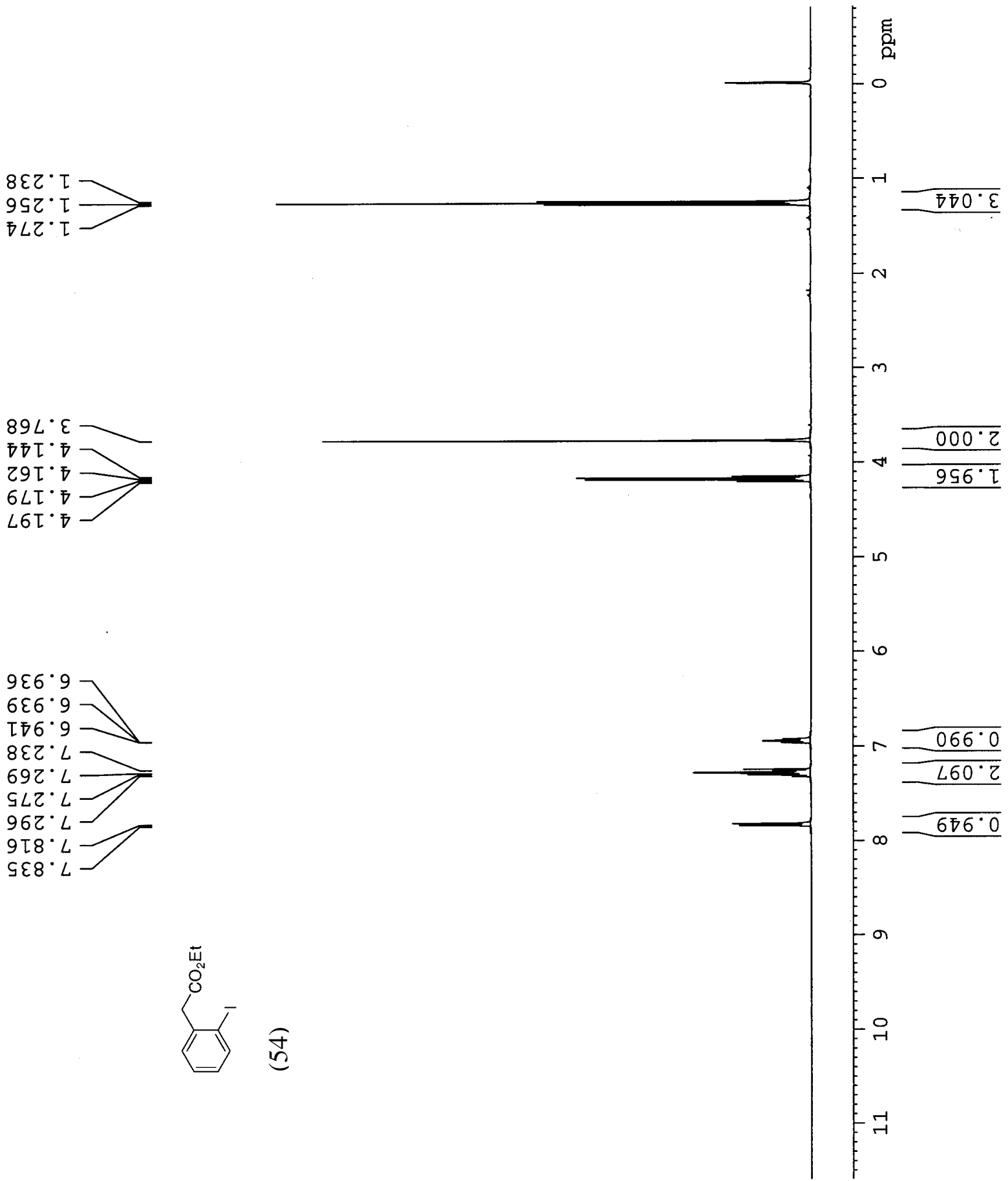


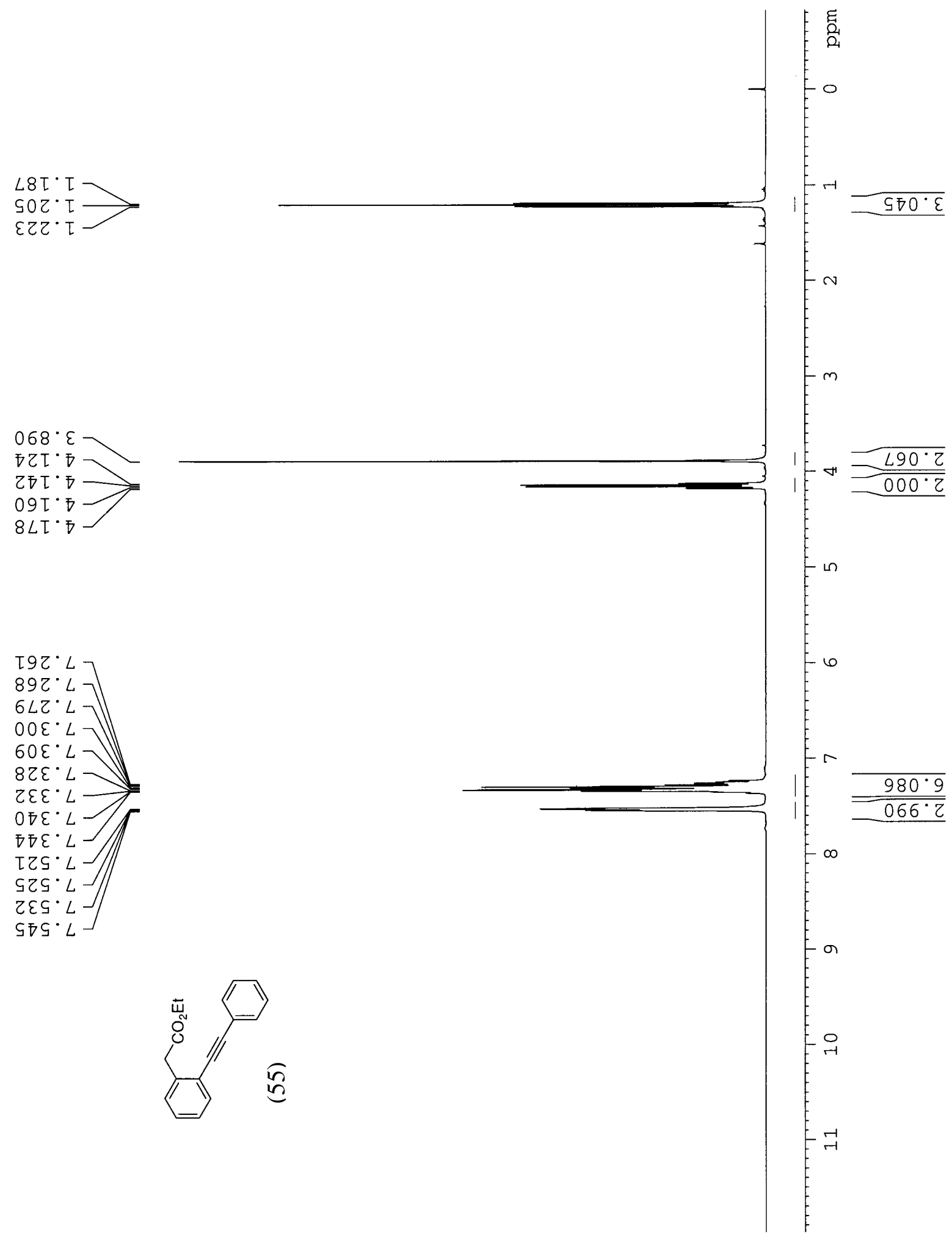


$\sigma \varepsilon 乙 \cdot \nabla \tau$

$9 \varsigma \varepsilon \cdot 07$

$Z \angle 6^{\circ} 09$

५โ8.9L

$\varepsilon \varepsilon[\cdot L L$

IS币 $L L$

ఐெ

ฤ $86^{\circ} \varepsilon 6$

$\varepsilon \varsigma て \cdot \varepsilon 乙 \tau$

$\varepsilon \sigma \varsigma^{*} \varepsilon Z \tau$
$L O Z \cdot L Z \tau$

$\angle Z$ LZ๐T

9S币.8ZT

$209^{\circ} 8 Z \mathrm{Z}$

$896^{\circ} 6 Z \tau$

$909^{\circ} \tau \varepsilon \tau$

$660^{\circ} \mathrm{Z \varepsilon \tau}$

$\angle L \nabla \cdot 9 \varepsilon \tau$

$\varsigma Z \varepsilon^{\cdot} \tau L \tau$

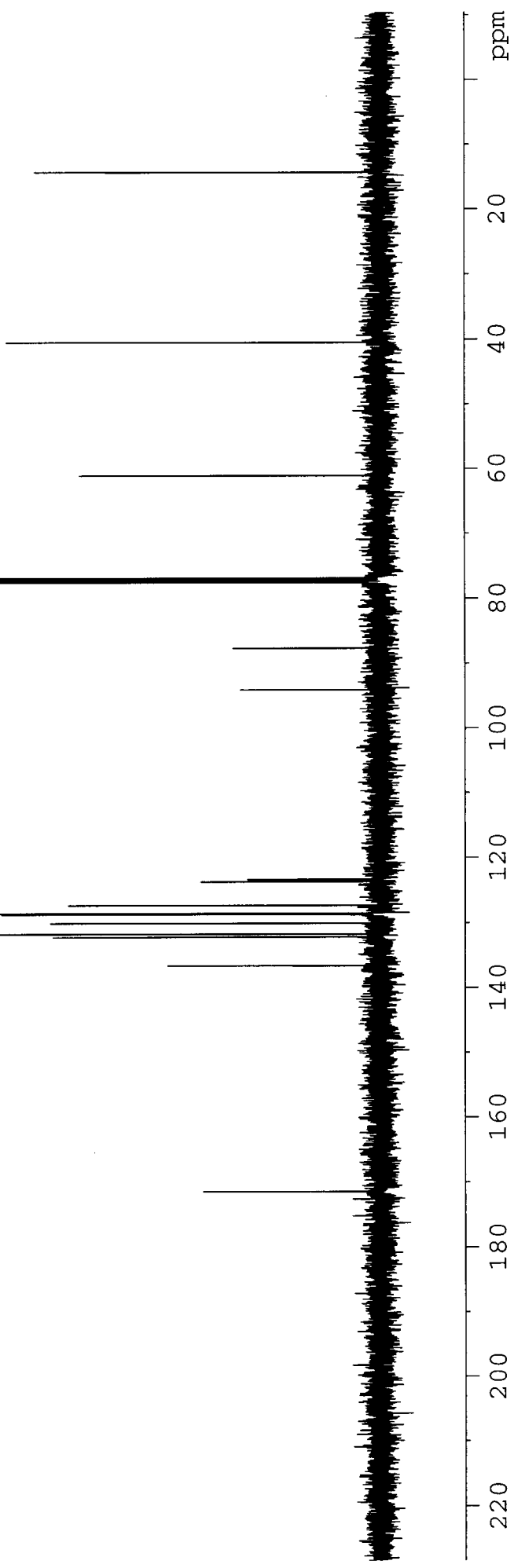



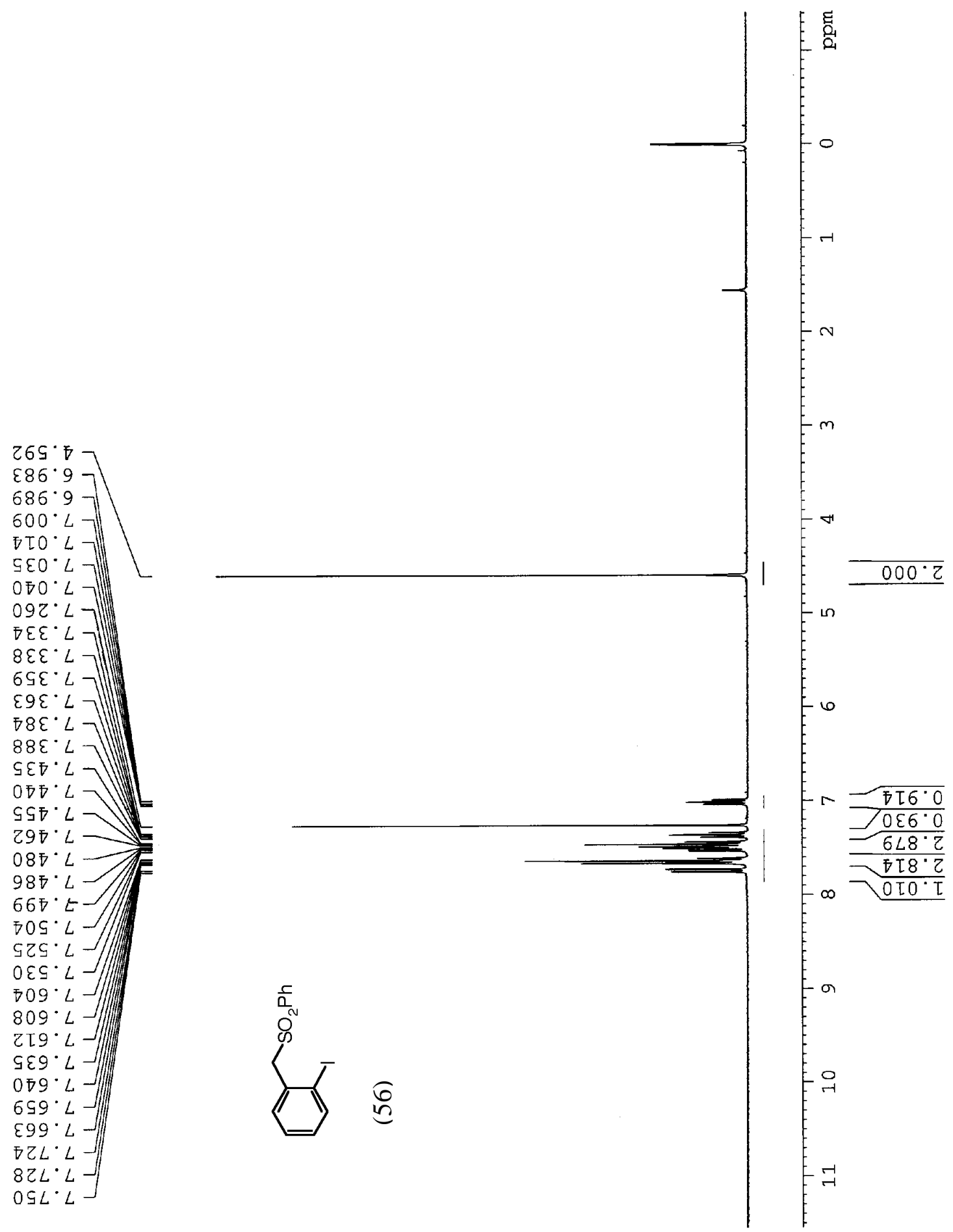


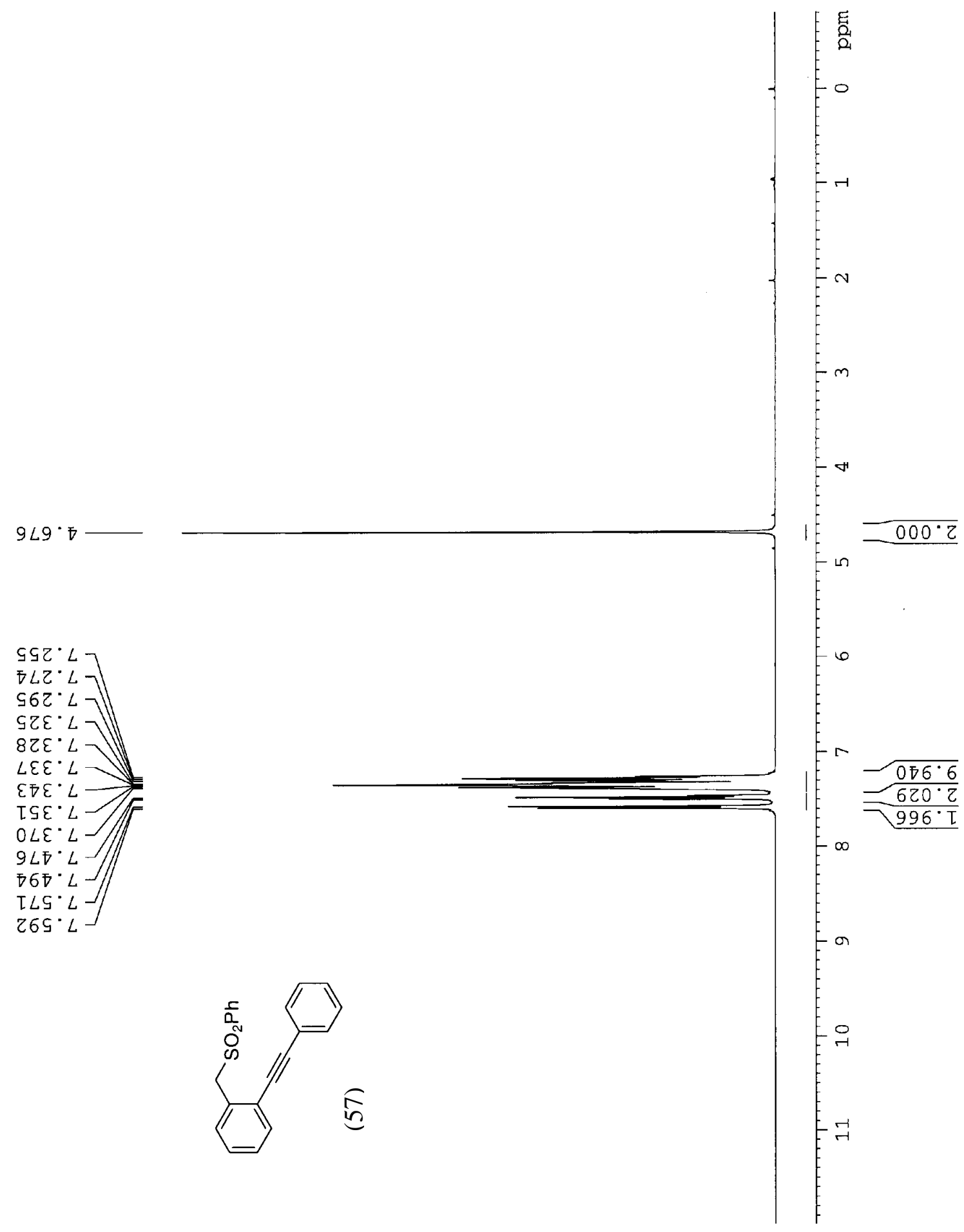



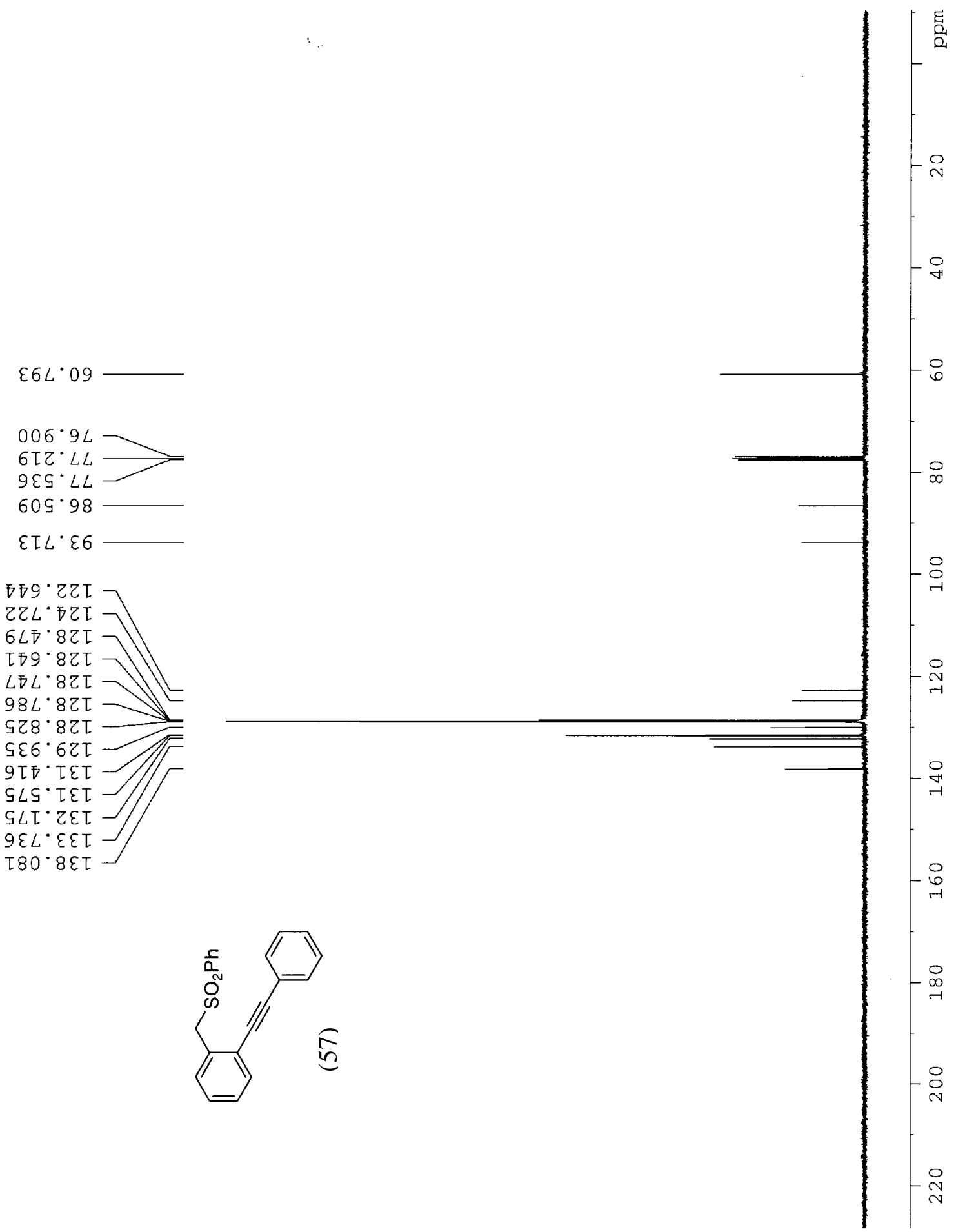


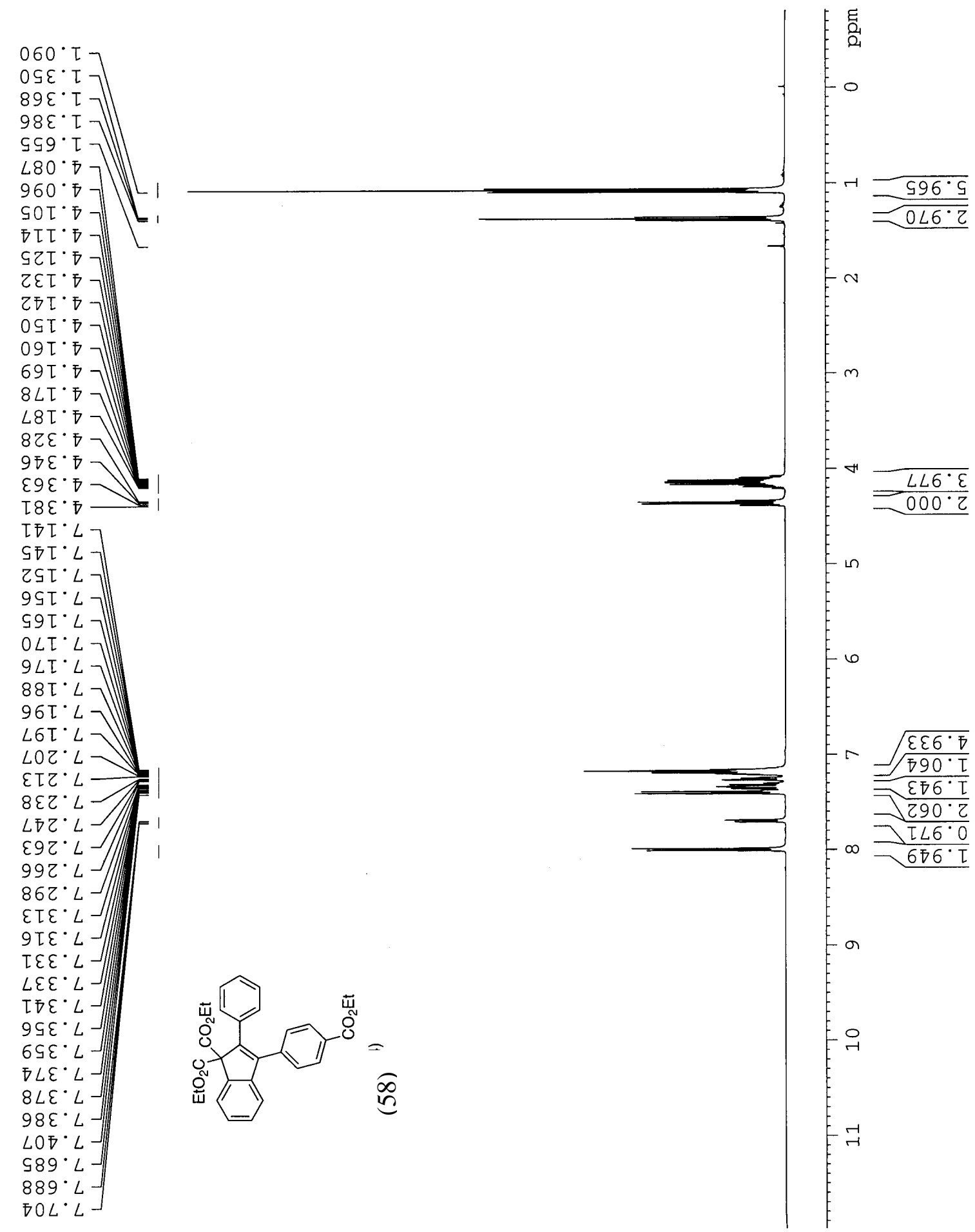




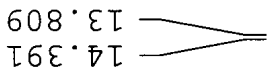

$7 \angle 0^{\circ}$ โ9

$9 L 6^{\circ} Z L$

T08.9L

6II' $L L$

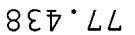
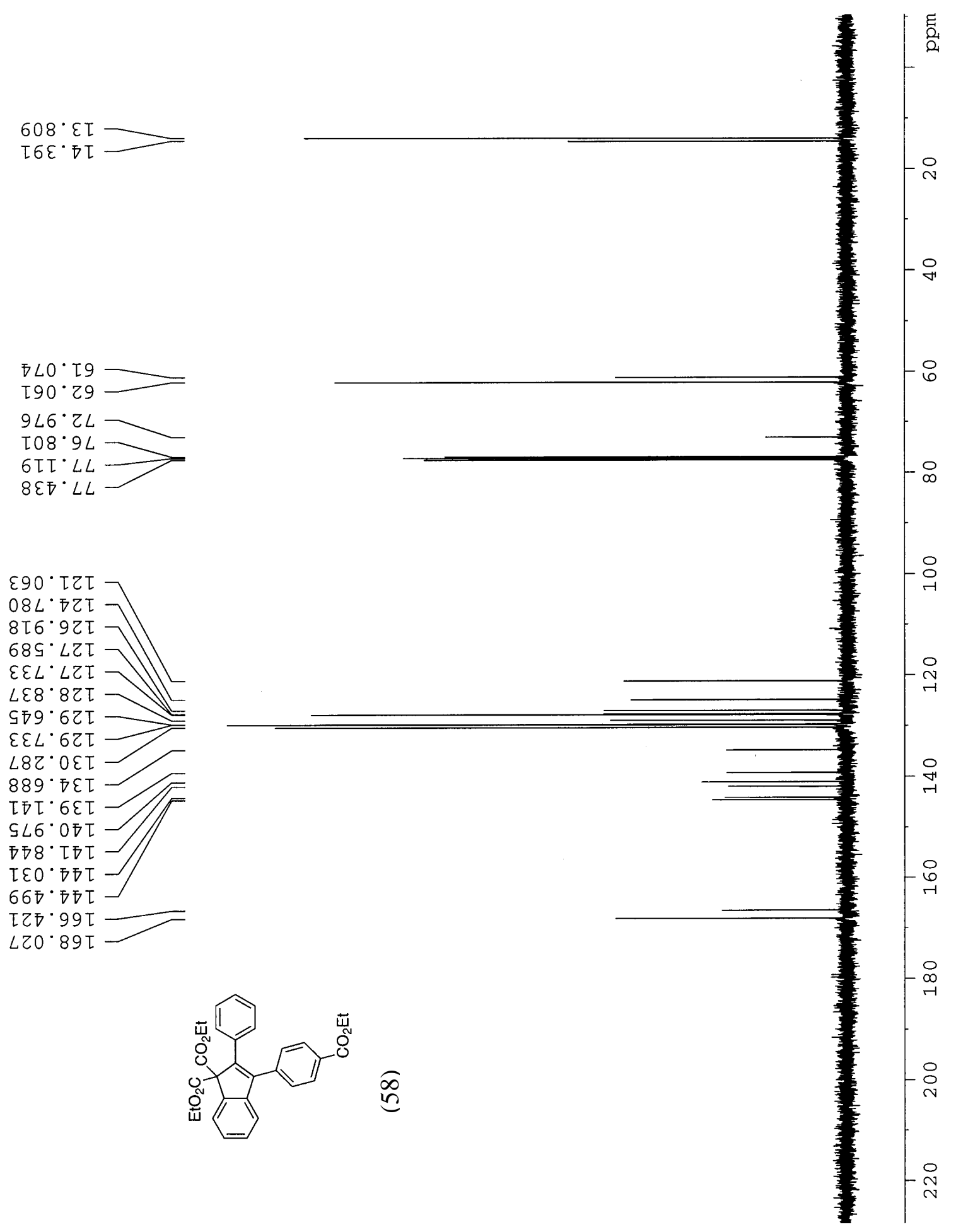


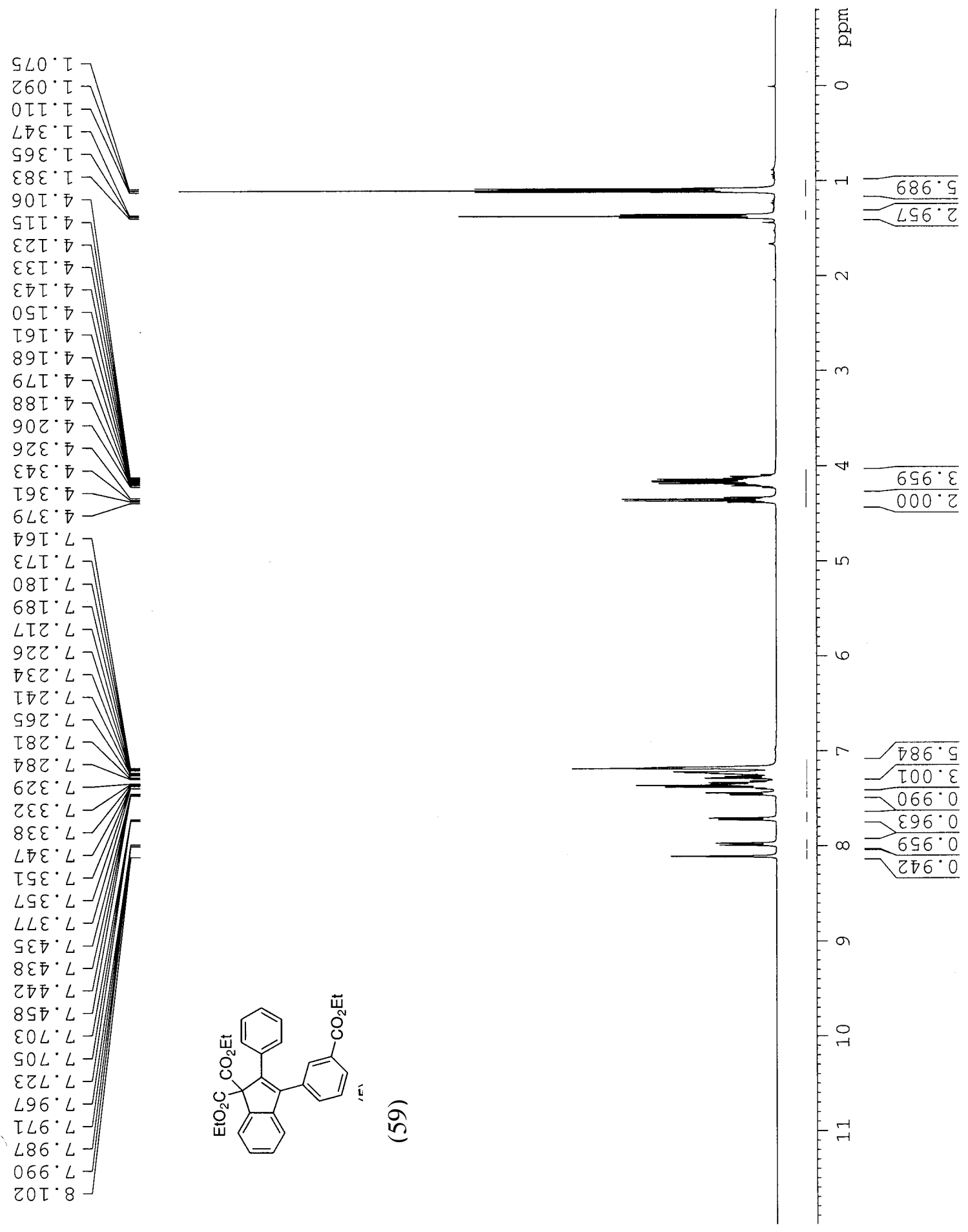


$Z Z 8^{\cdot} \varepsilon I \longrightarrow$

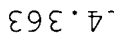

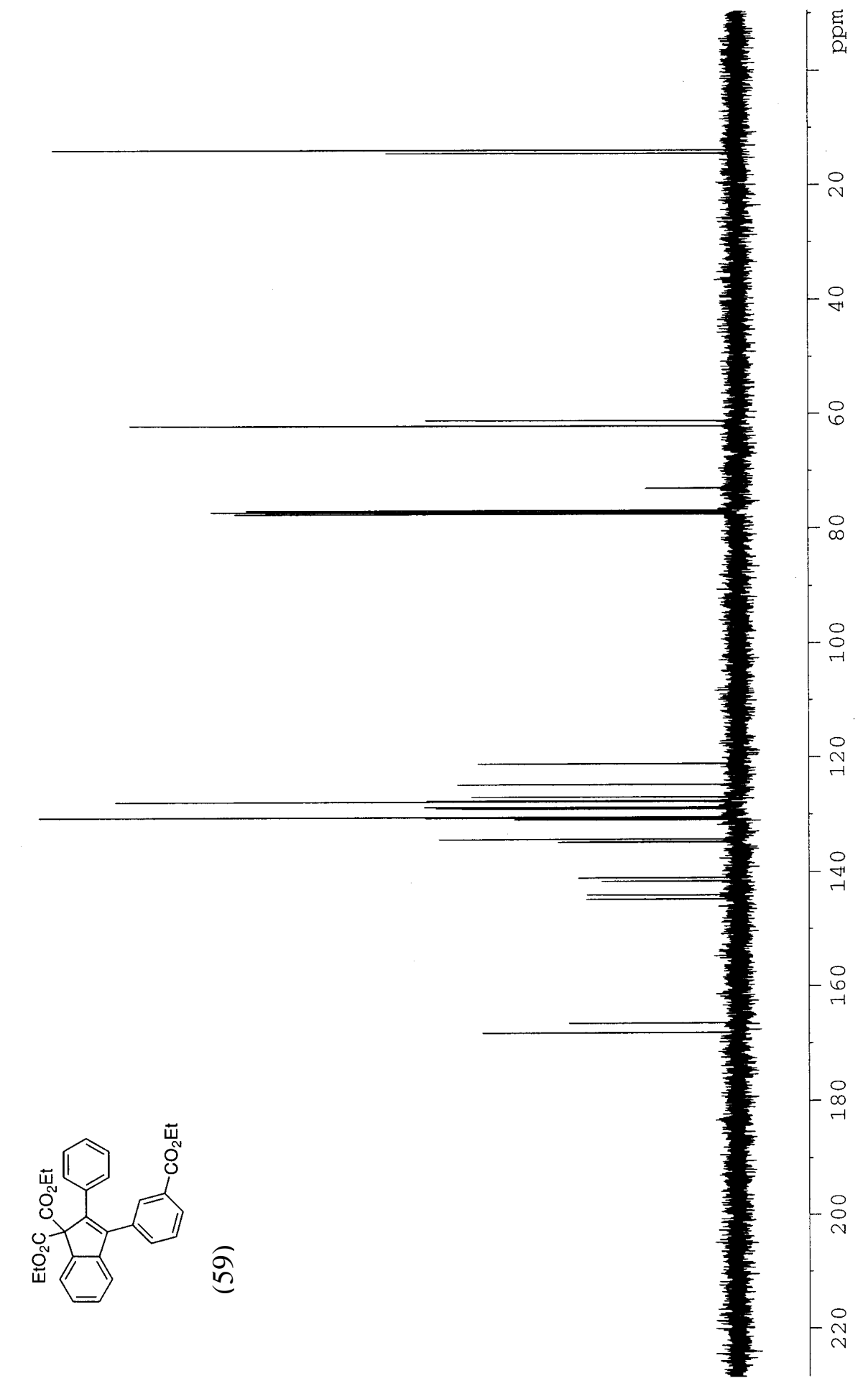

TOL' T9

$820 \cdot 29$

TE6. $2 L$

$808^{\circ} 9 L$

$9 Z I^{\circ} L L$
$9 \nabla \nabla \cdot L L$

โ80. โZ

STL・ 万ZT

IL8.9Z

$769^{\circ} \angle Z T$

与ฤ $9 \cdot 8 乙$

$\varepsilon \nabla 8.8 Z \tau$

५५6.8乙T

$8 \varepsilon \varepsilon^{\circ} 0 \varepsilon \tau$

905. OहT -

6โ8. ०हा

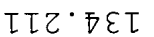

$\angle Z 9^{\circ} \nabla \varepsilon \tau$

$9 Z L \cdot \nabla \varepsilon \tau$

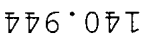

EZS. IDI

$\bar{\nabla} 6^{\circ} \varepsilon \nabla T$

S $\angle 9^{\circ} \nabla \nabla T$

$807 \cdot 99 T$

$960 \cdot 89 \mathrm{I}$ 


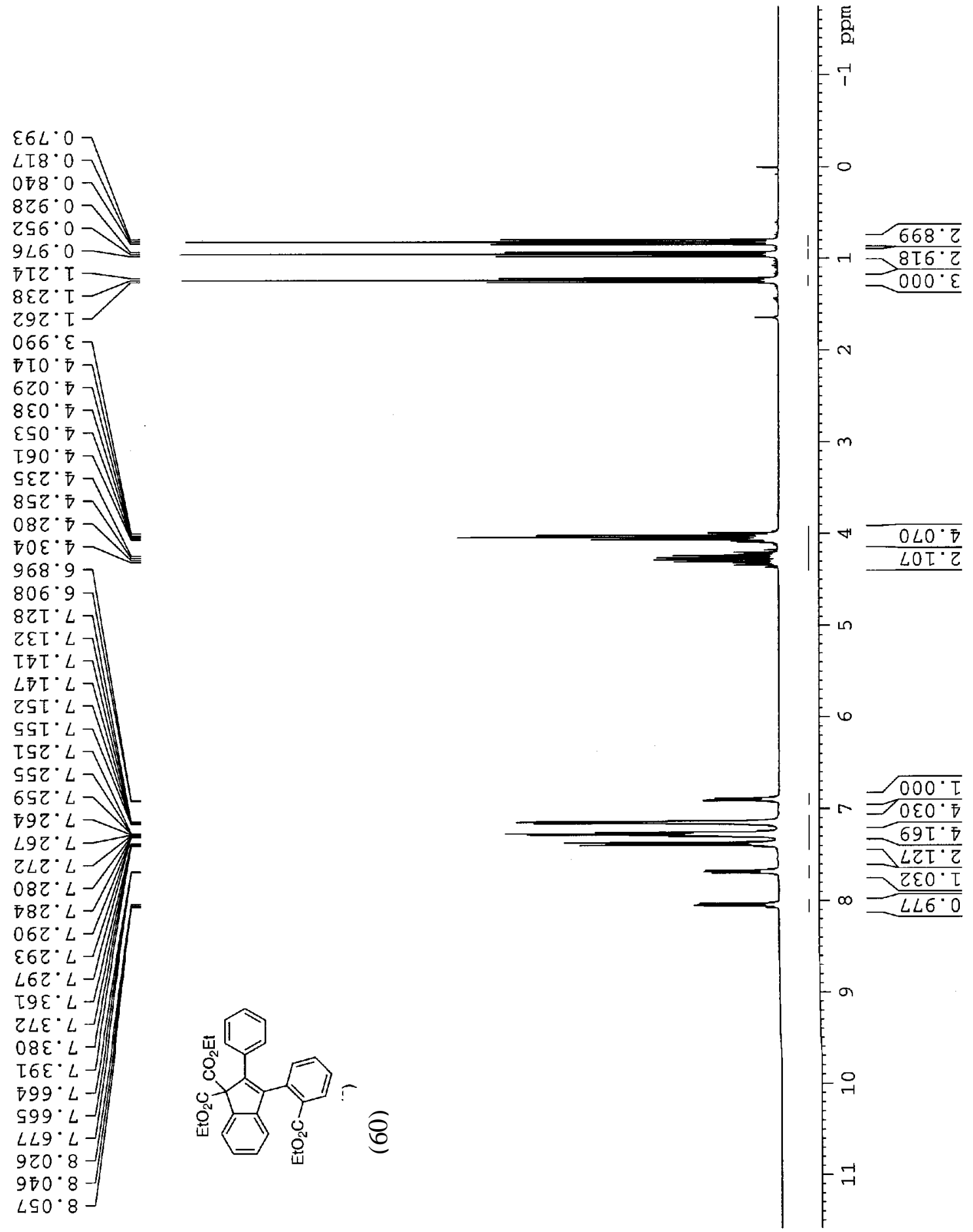




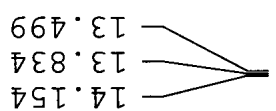

296. 29

06L T9

$09 L^{\circ} \mathrm{ZL}$

$678 \cdot 9 L$

$\varepsilon L Z^{\circ} L L$

$\angle 69^{\circ} L L$

$065^{\circ} 02 \mathrm{~T}$

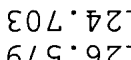

$60 \bar{D} \cdot \angle Z T$

$8 T L^{\circ} \angle Z I$

$8 \bar{T} \cdot 8 Z T$

$998^{\circ} 8 \mathrm{Z}$

โOZ. OET

$628^{\circ} 0 \varepsilon \tau$

Ђ0 ${ }^{\circ}$ โ $\tau$ โ

Ђ乙8 โहโ

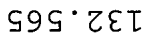

$\varepsilon 6 \tau \cdot \varsigma \varepsilon \tau$

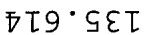

$\overline{D L G} 6 \varepsilon \tau$

8โ万. $07 \tau$

6โ0.9ரT

โZT.9ரT

$\angle 89^{\circ} \angle 9 T$

$9 \varepsilon L^{\circ} \angle 9 T$

SOI. $69 \mathrm{I}$

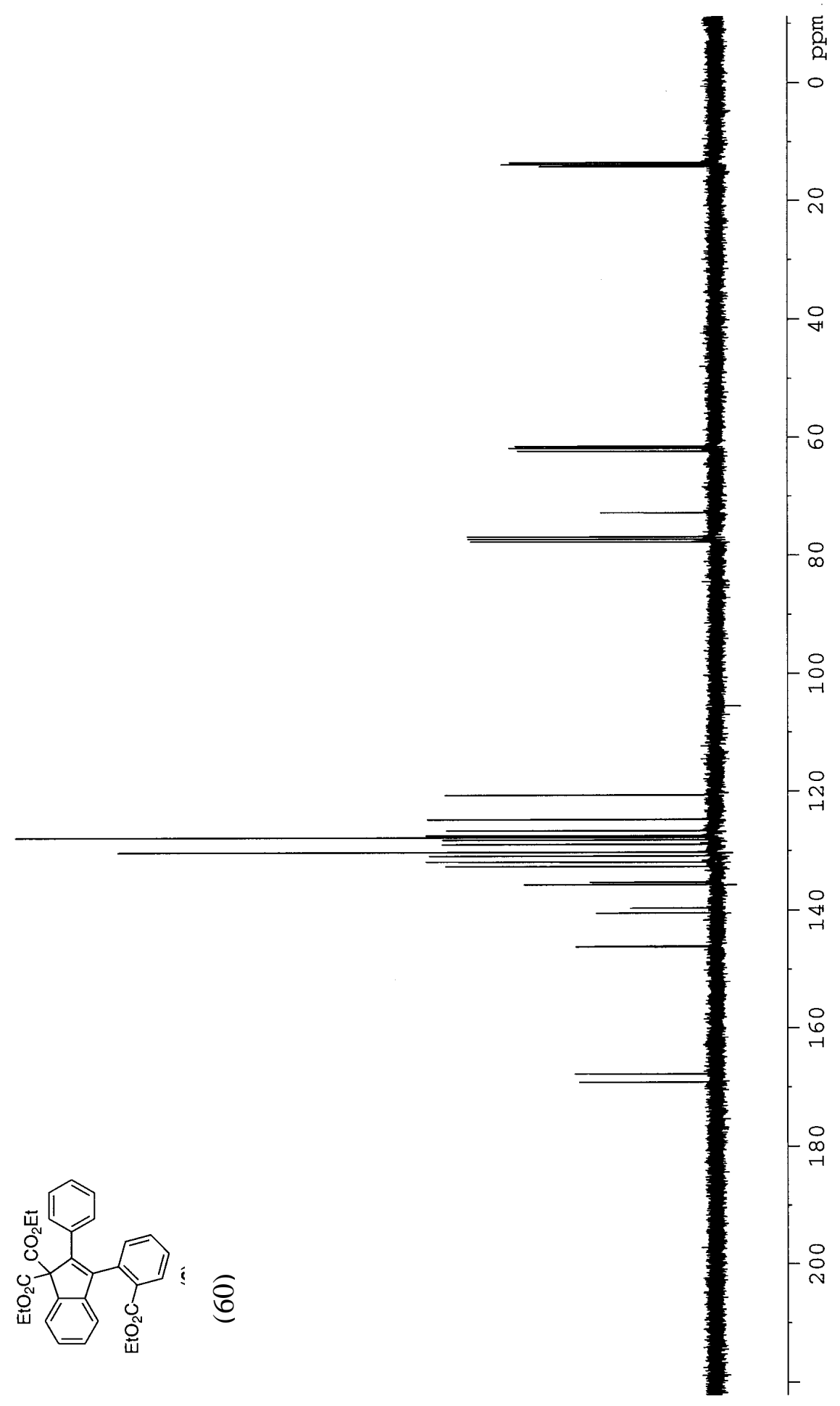




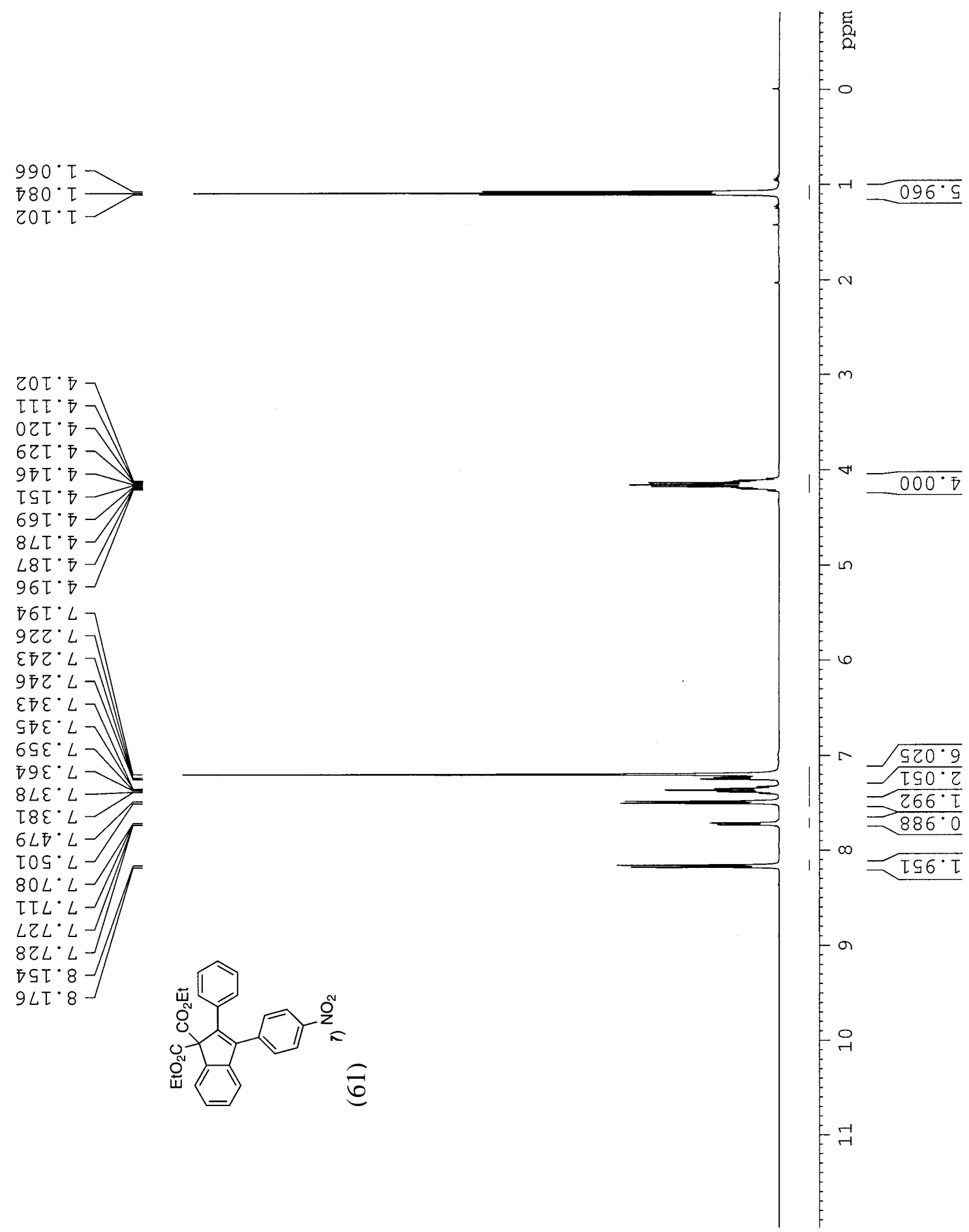


$\tau 08^{\circ} \varepsilon \tau$

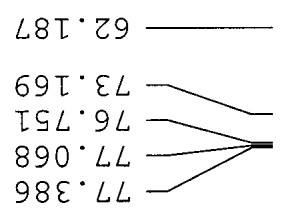

$6 L L \cdot 0 Z I$

$86 L \cdot \varepsilon Z$

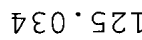

$8 \varepsilon \mathrm{C}^{\circ} \mathrm{LZT}$

$\angle Z 6^{\circ} \angle Z T$

$0 \angle 6^{\circ} \angle Z T$

$796^{\circ} 8 Z \mathrm{~T}$

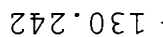

$889^{\circ} 0 \varepsilon \tau$

$98{ }^{\circ} \nabla \varepsilon \tau$

L $\sigma^{\circ}$ OळI

$68 \varepsilon^{\circ}$ 切

$626^{\circ}$ 乙๐

$\varsigma \varepsilon \tau \cdot \varepsilon \nabla \tau$

乙โ8.ह戸ٓ

$\angle \varepsilon{ }^{\circ} \angle \nabla \tau$

DOL L $L 9 T$

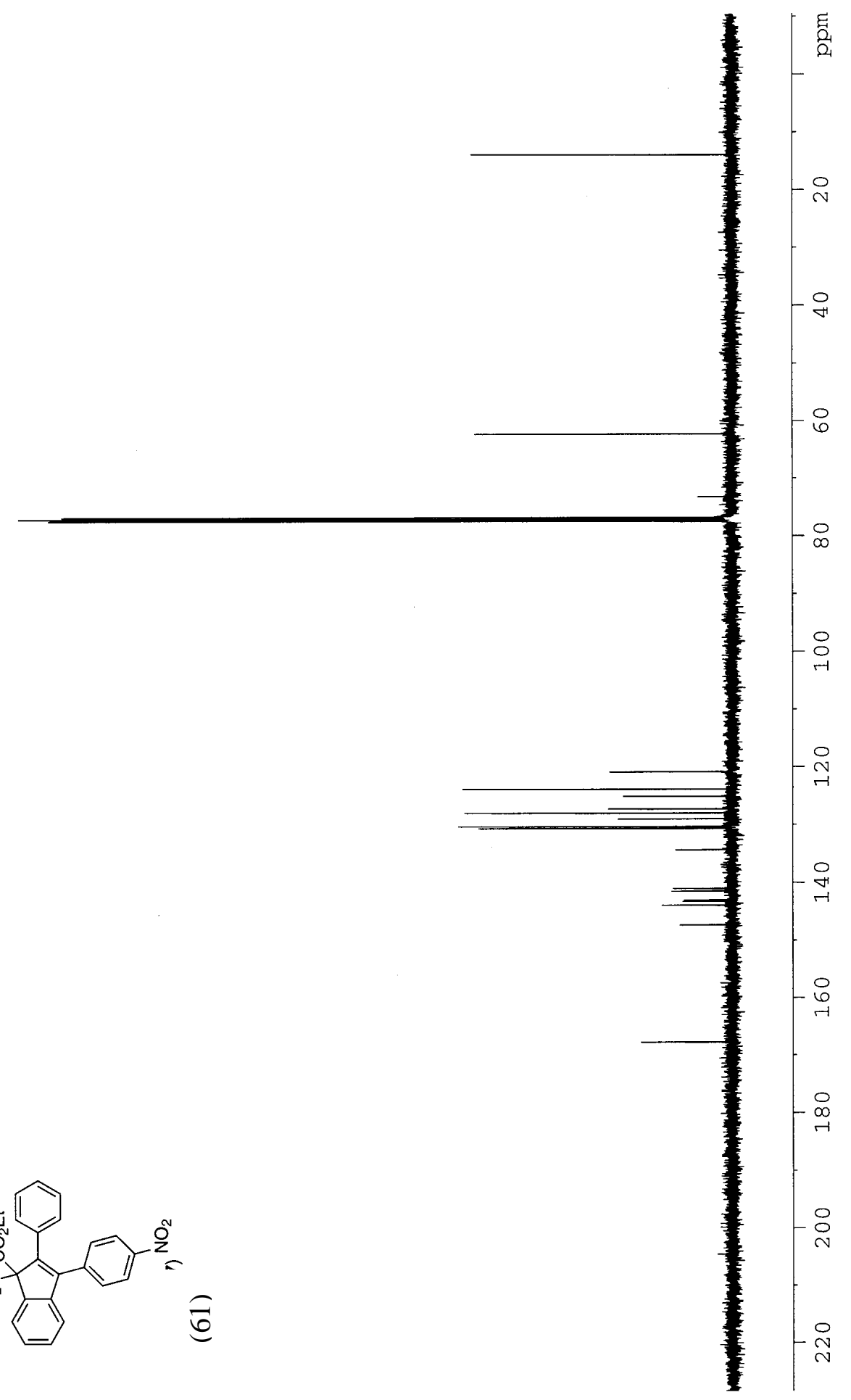


$Z \angle O^{\circ} I$

$680^{\circ} \mathrm{T}$

$\angle O L \cdot T$
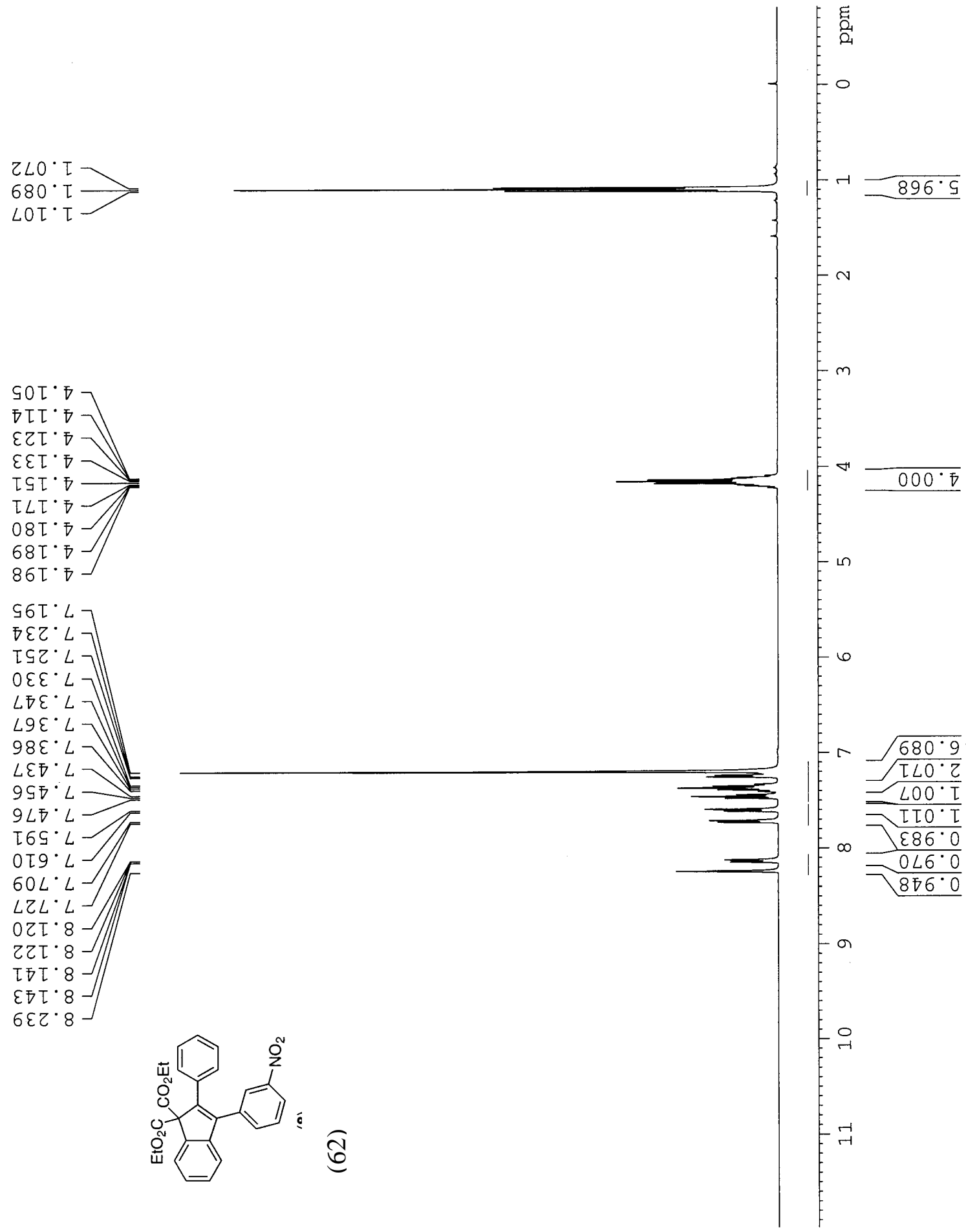

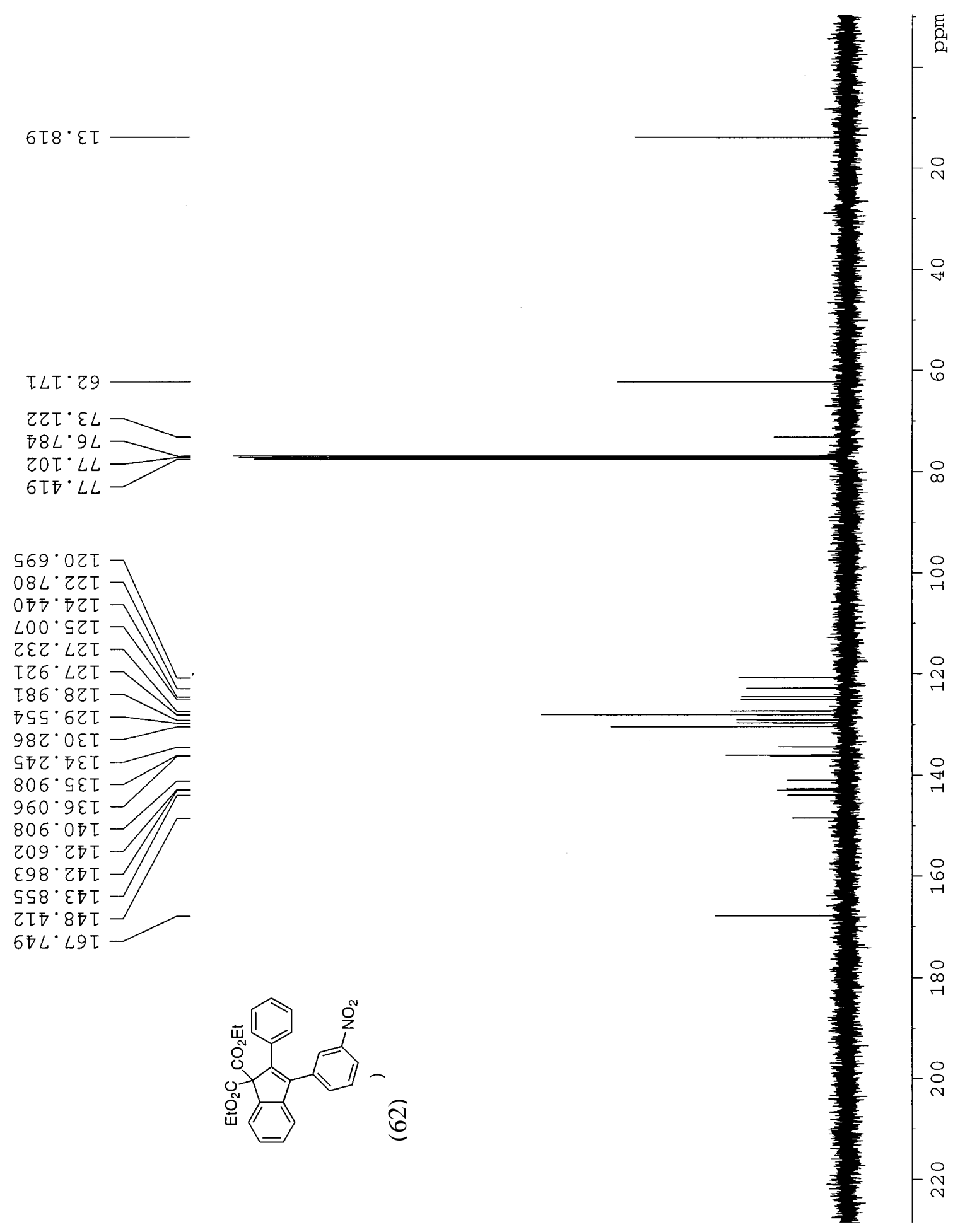


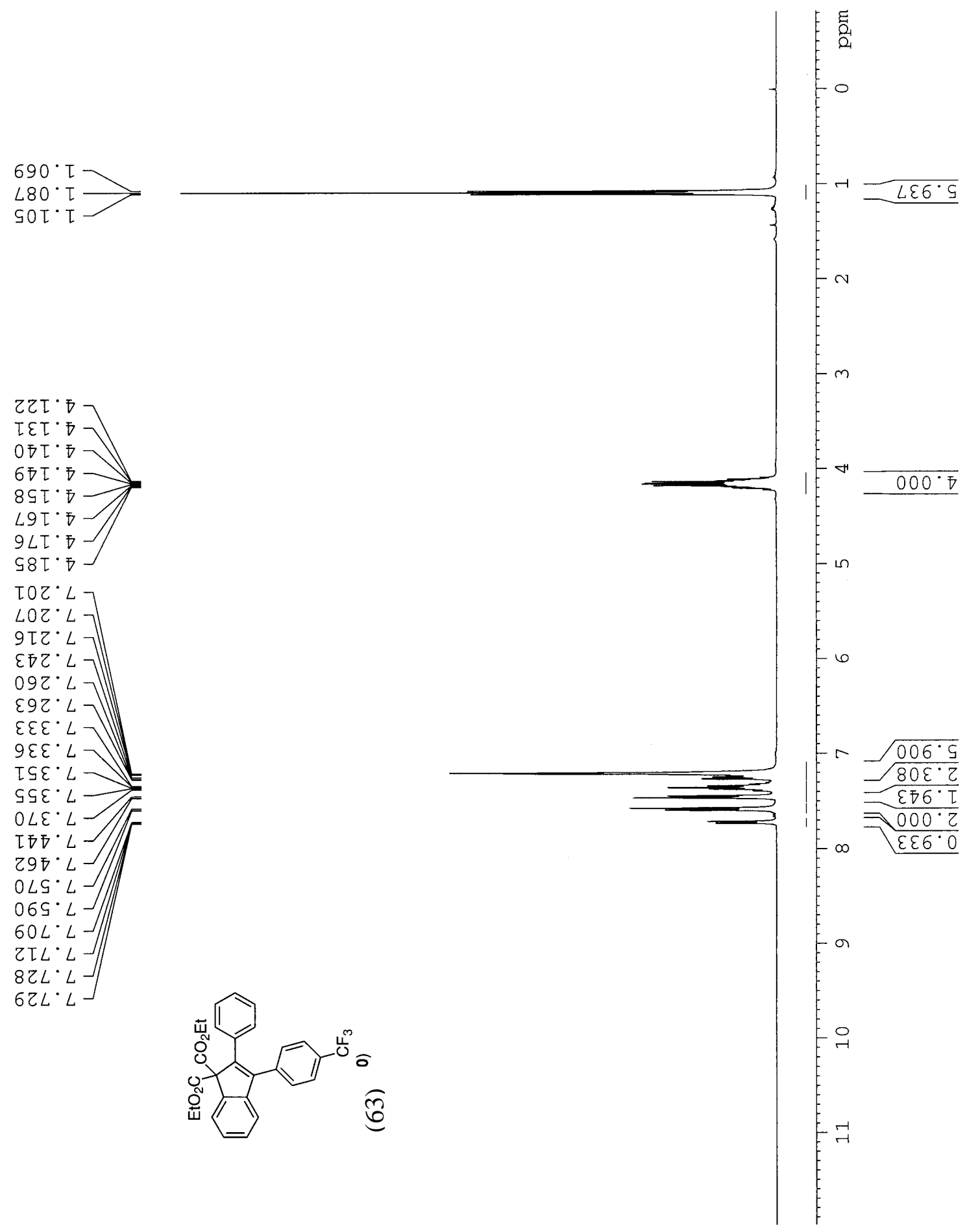




\section{$908^{*} \varepsilon \tau$}
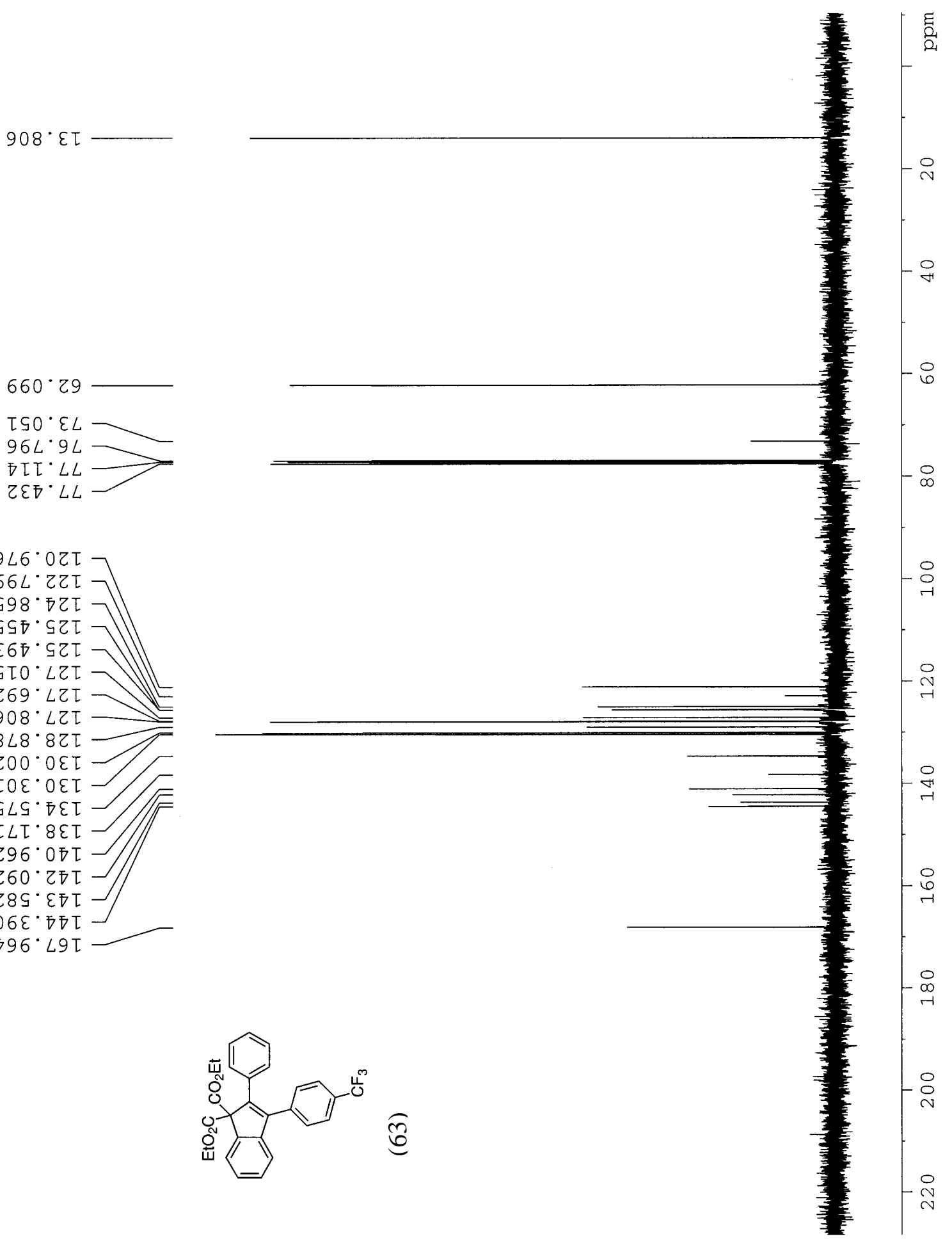

$9 \angle 6^{\circ} 0 z$

$66 L^{\circ} \mathrm{ZZT}$

ร98

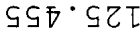

$\varepsilon 67 \cdot \varsigma ⿱ 一$

ST0 $\angle Z T$

$269^{\circ} \angle Z T$

$908^{\circ} \mathrm{LZT}$

$8 \angle 8^{\circ} 8 Z \tau$

乙०००० OET

Т०E. Oहा

५LS. $ธ \varepsilon \tau$

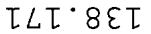

$296^{\circ} 0 \bar{T}$

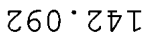

Z८५. $\varepsilon \nabla T-$

$06 \varepsilon^{\circ} \nabla \nabla \tau$

$796^{\circ} \angle 9 T$ 


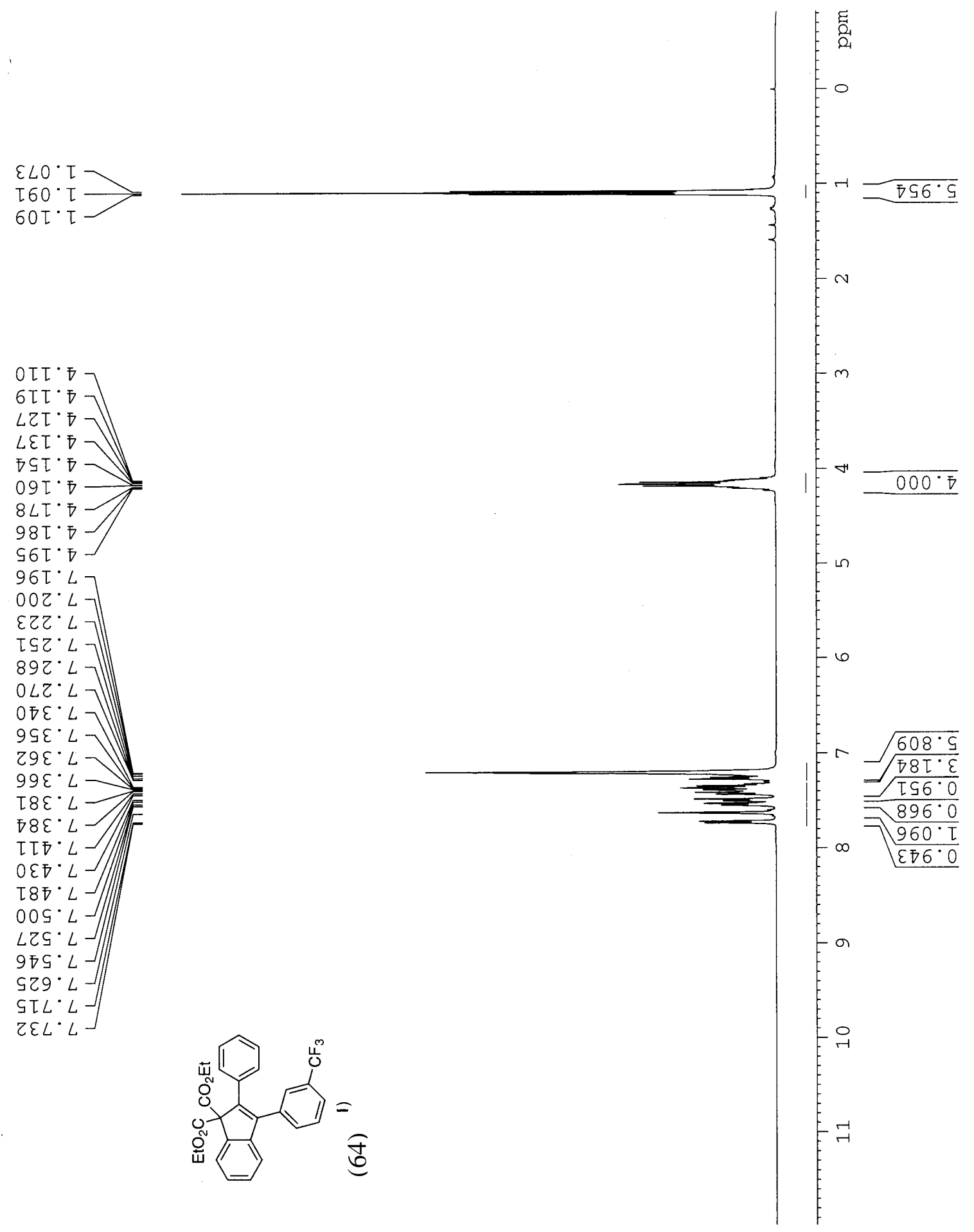


$\varepsilon \tau 8^{*} \varepsilon \tau$
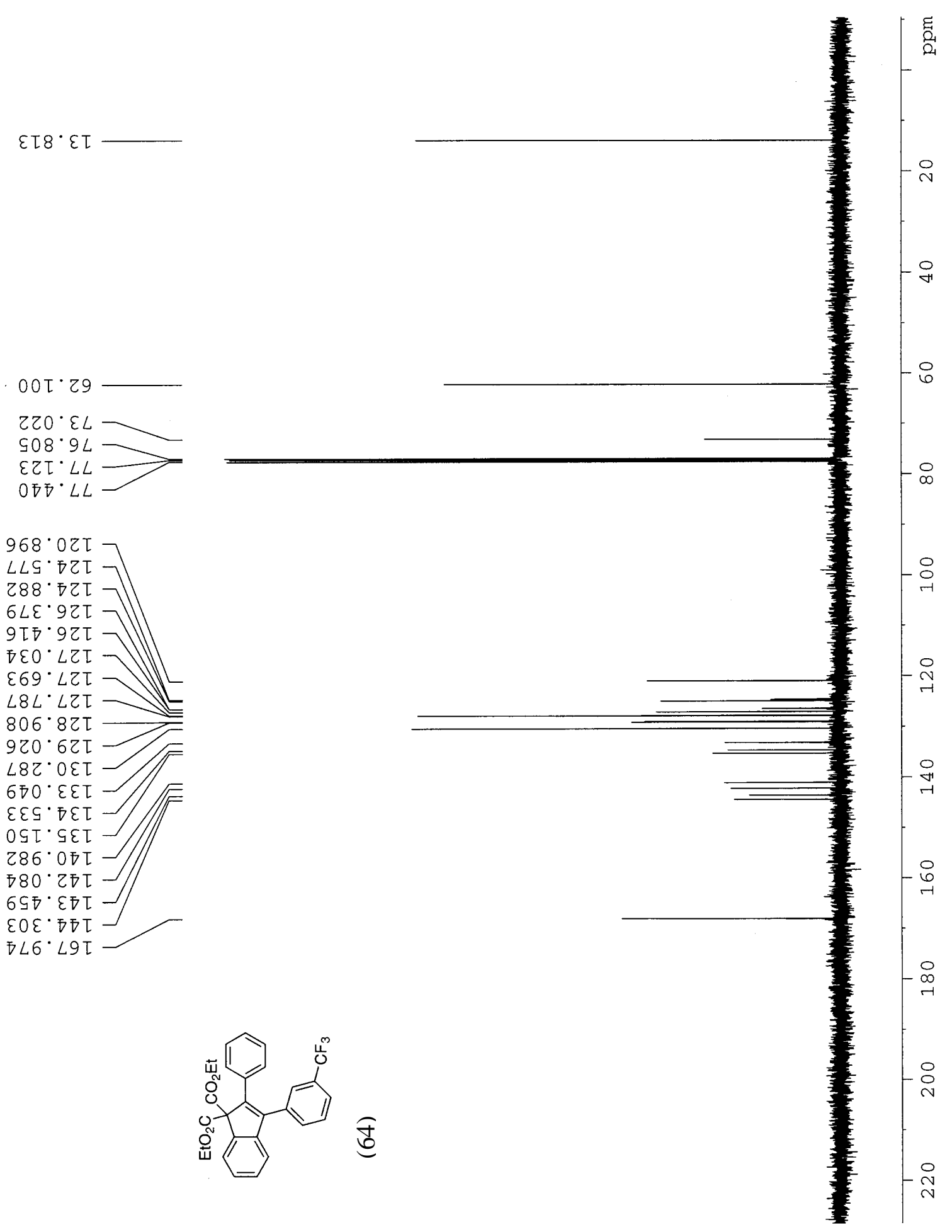


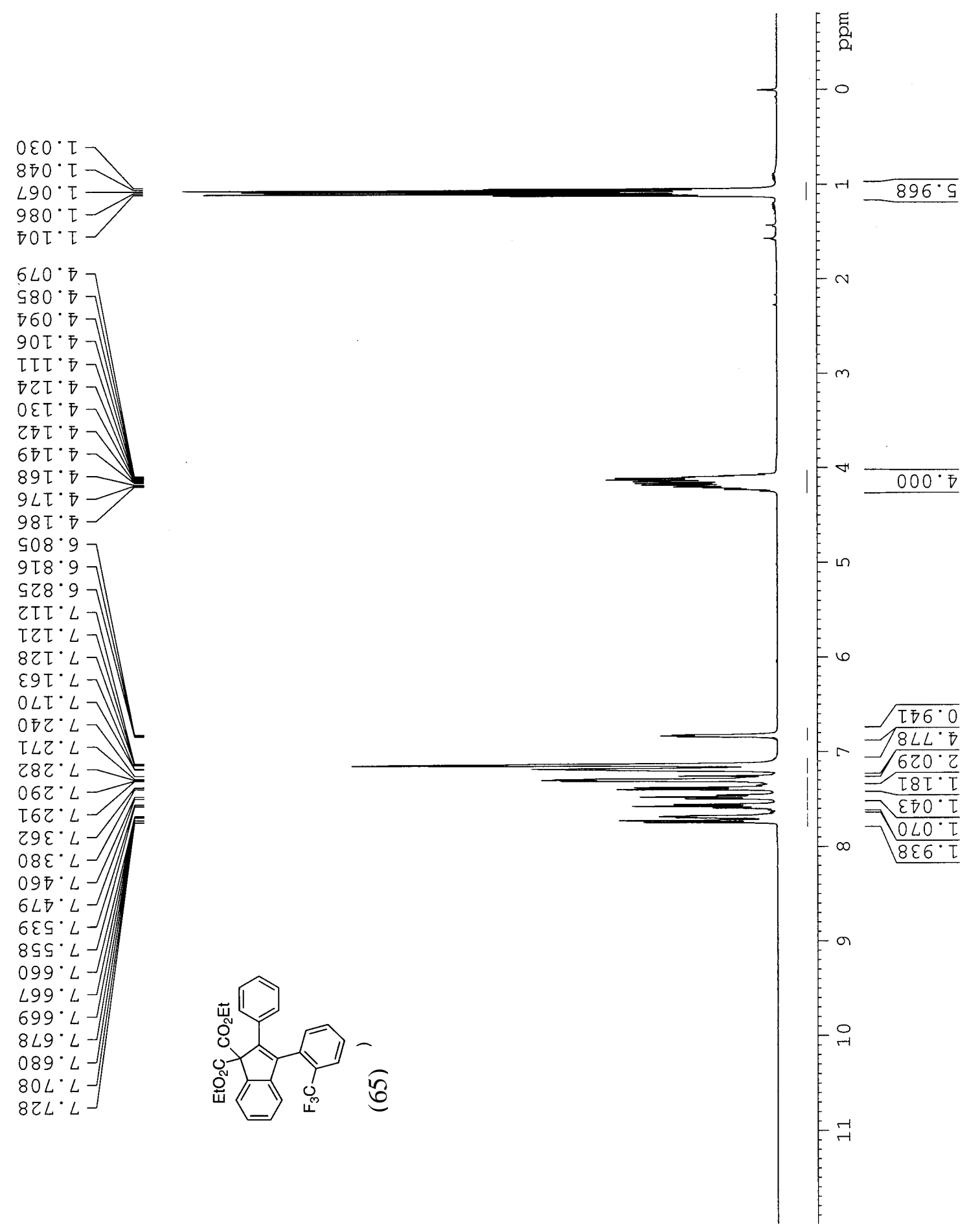



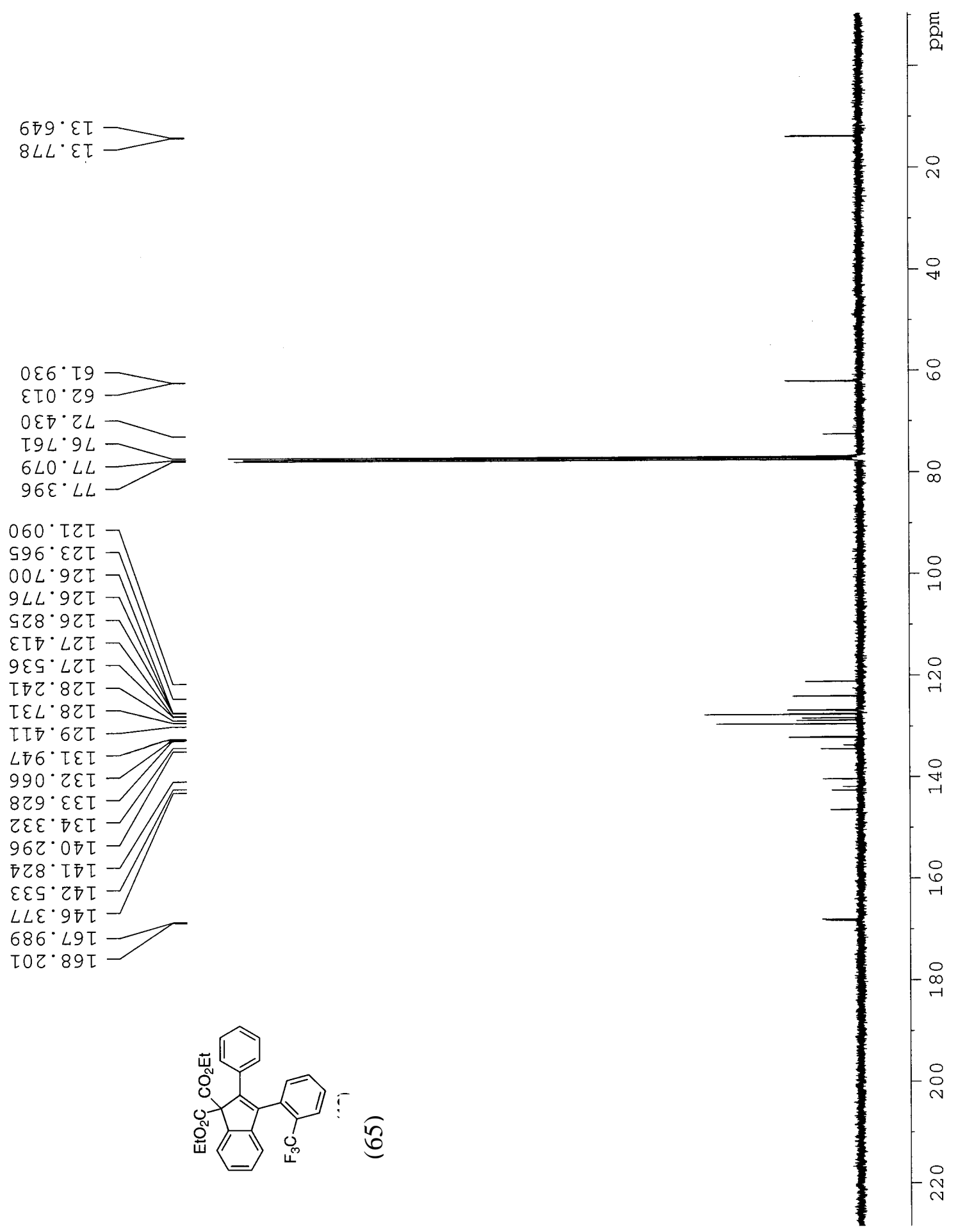


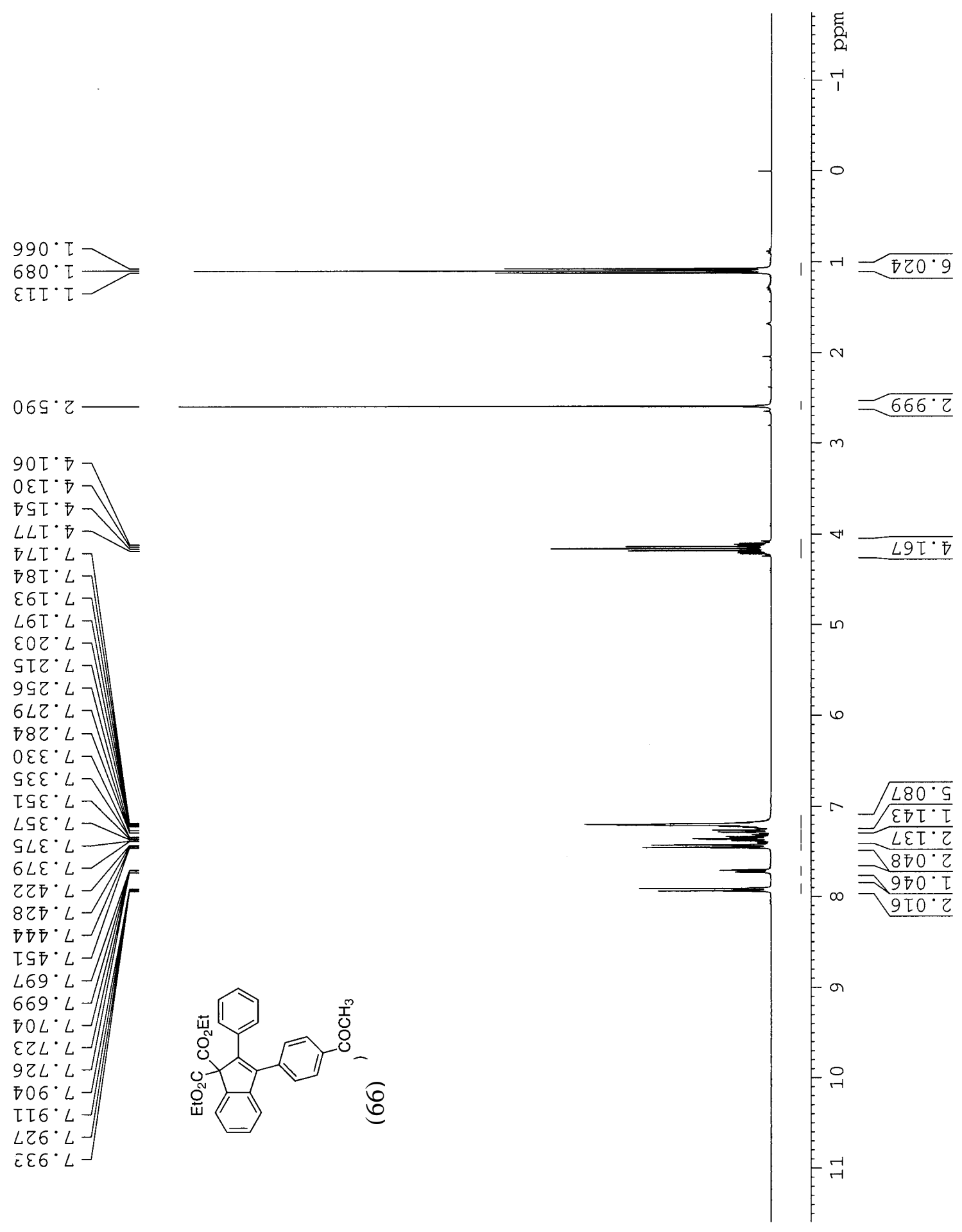


$\varepsilon 08^{\cdot} \varepsilon \tau$

$\angle ฐ 9 \cdot 9 Z$

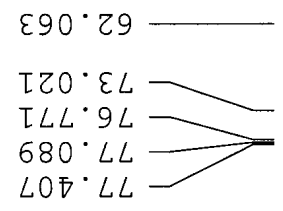

$820^{\circ} \tau z$

6โ8 จ乙

8๐6.9Z

SZ9. $\angle Z T$

$8 \nabla L \cdot \angle Z T$

ZZS.8ZI

งह8.8ZT

$088^{\circ} 6 Z \mathrm{I}$

$88 \mathrm{Z}^{\circ}$. $\varepsilon \mathrm{I}$

๑8 $จ \varepsilon \tau$

$\angle 82 \cdot 9 \varepsilon \tau$

$997 \cdot 6 \varepsilon \tau$

$\varepsilon 86^{\circ}$ ODT

$500^{\circ} 27 \tau$

$906^{\circ} \varepsilon \nabla \tau$

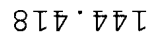

T $\angle 6 \cdot \angle 9 T$

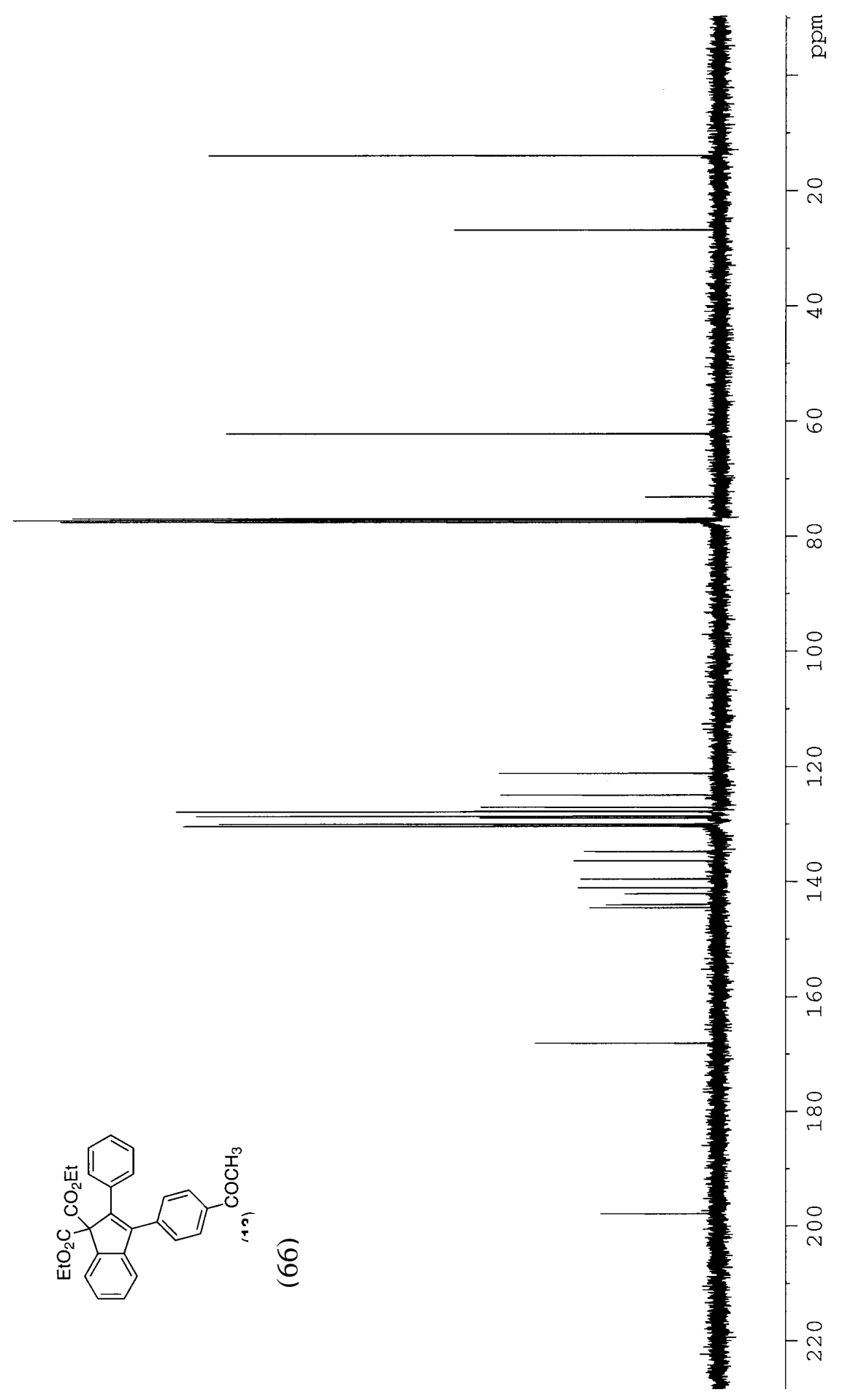

$\angle \nabla L \cdot \angle 6 T$

6 


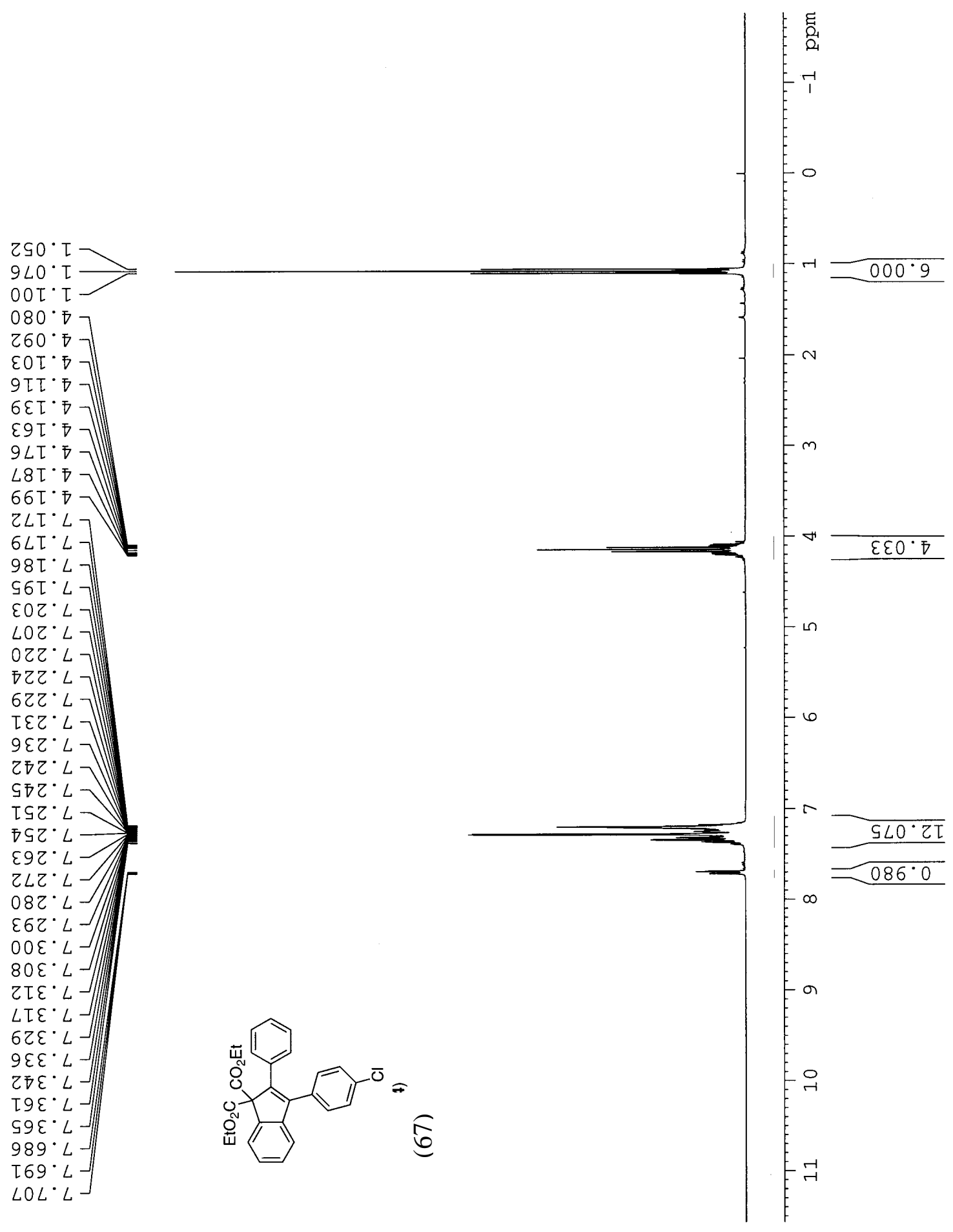


OTO. 万T
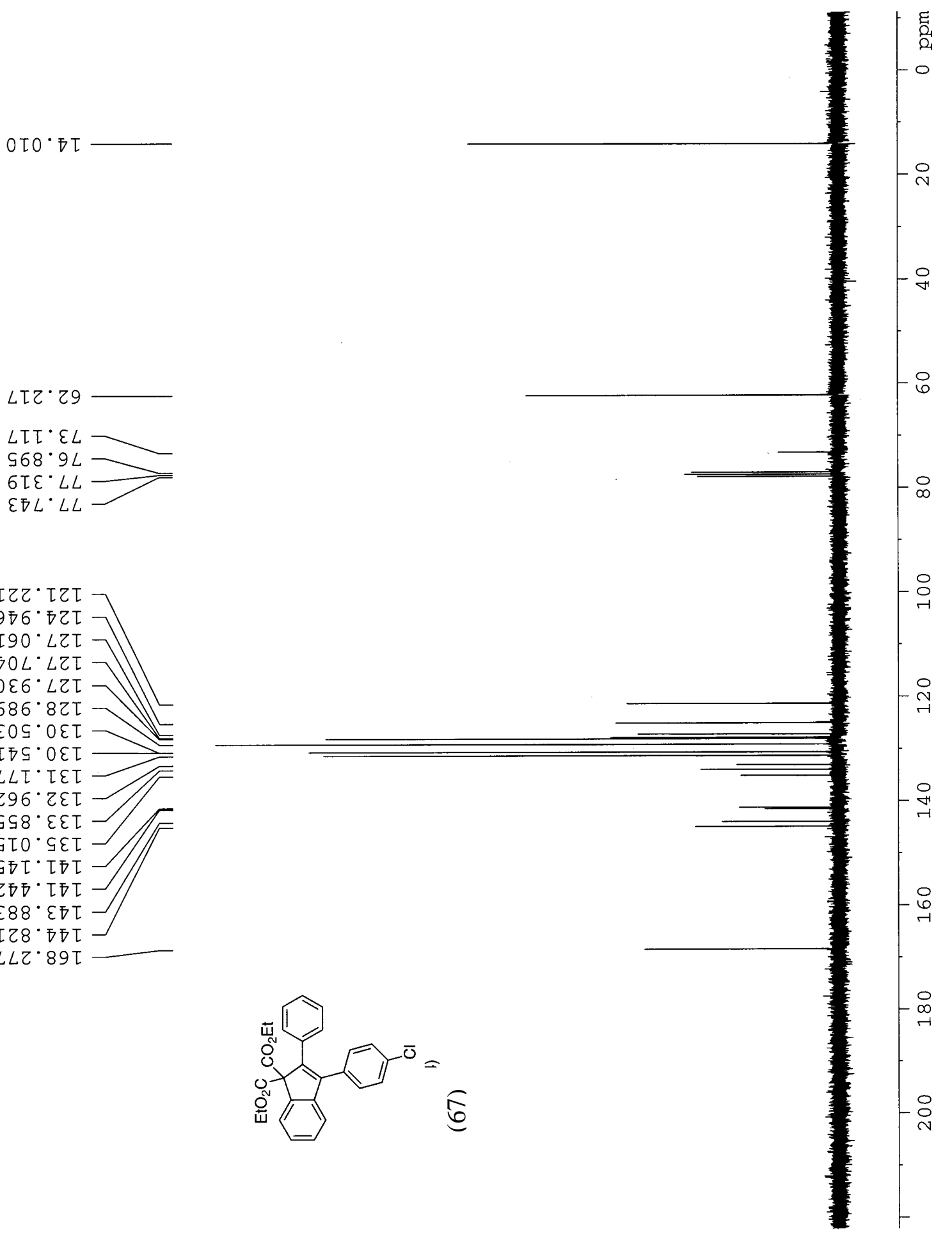

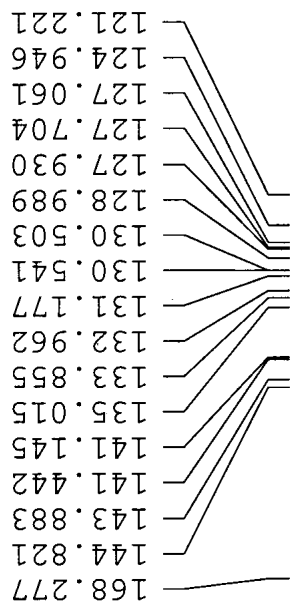




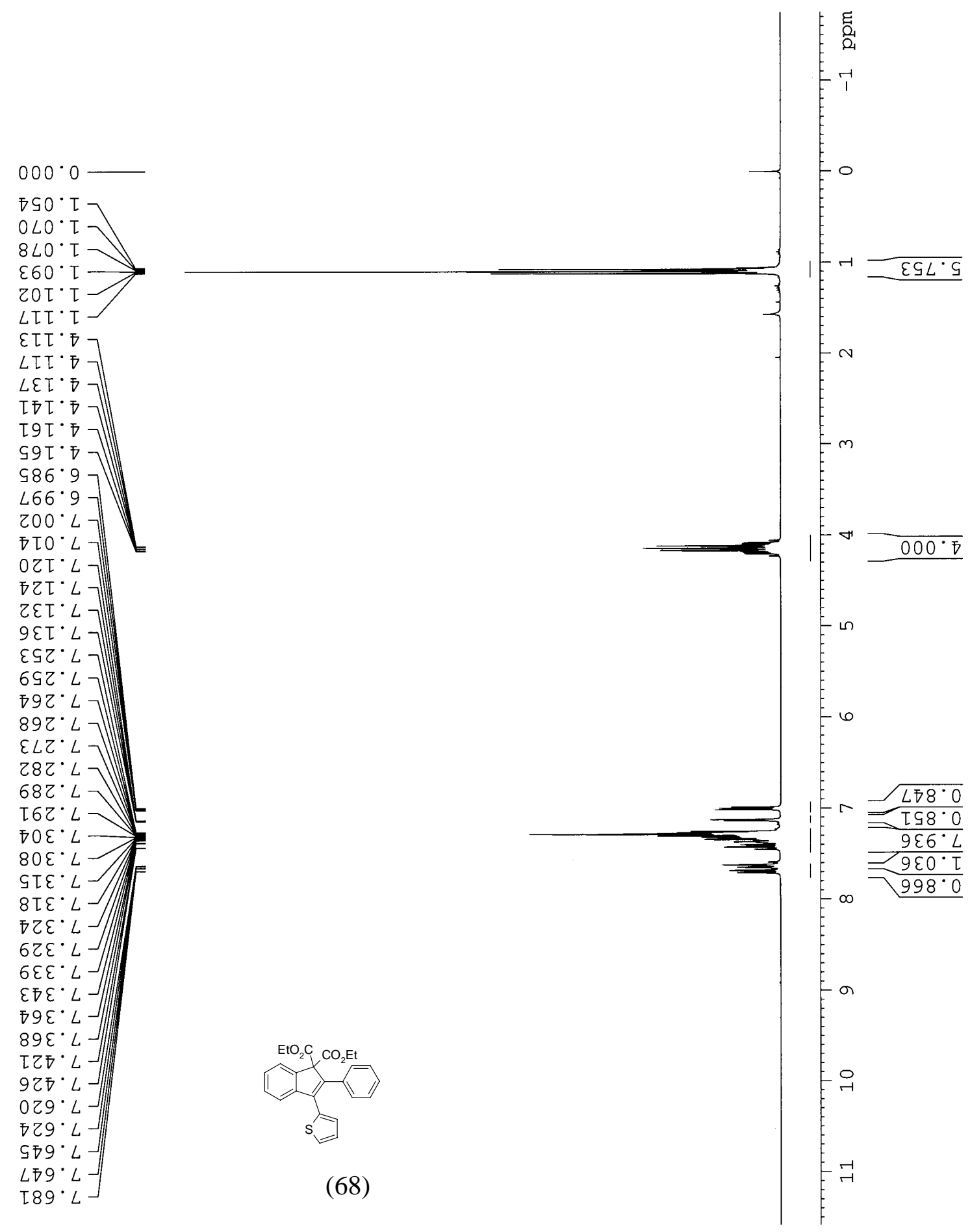



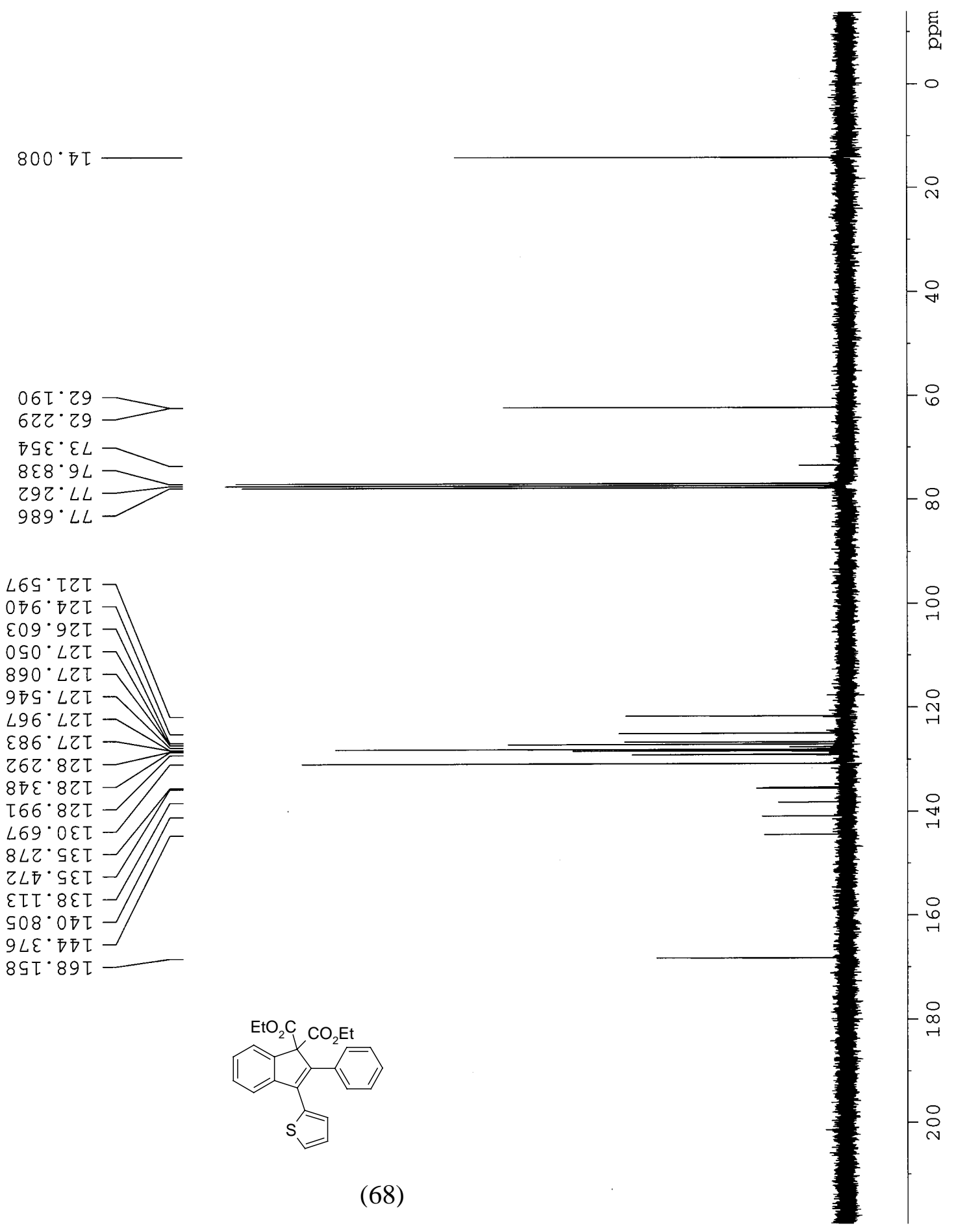

$8 \mathrm{~S} \cdot 89 \mathrm{~T}$

(68) 


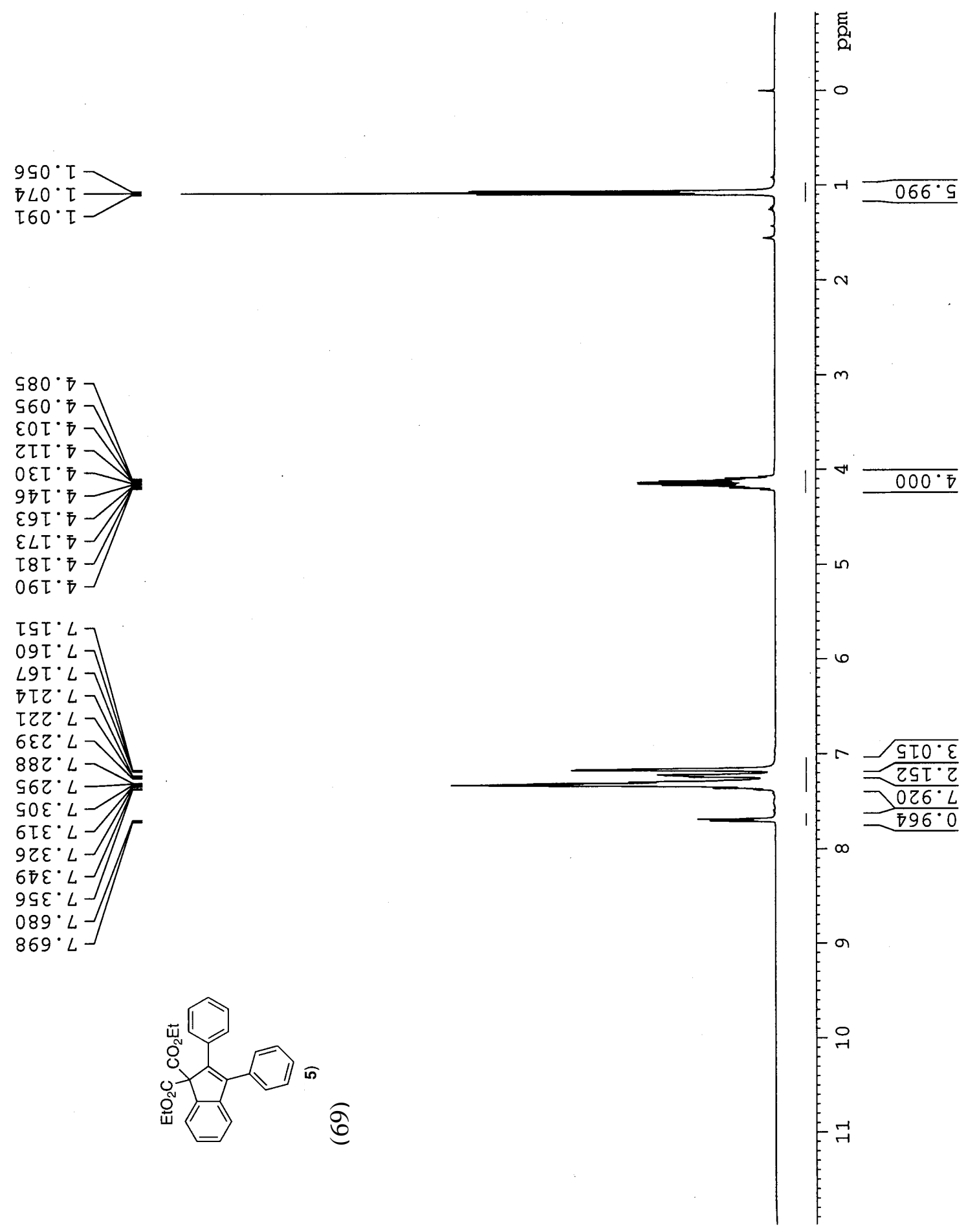



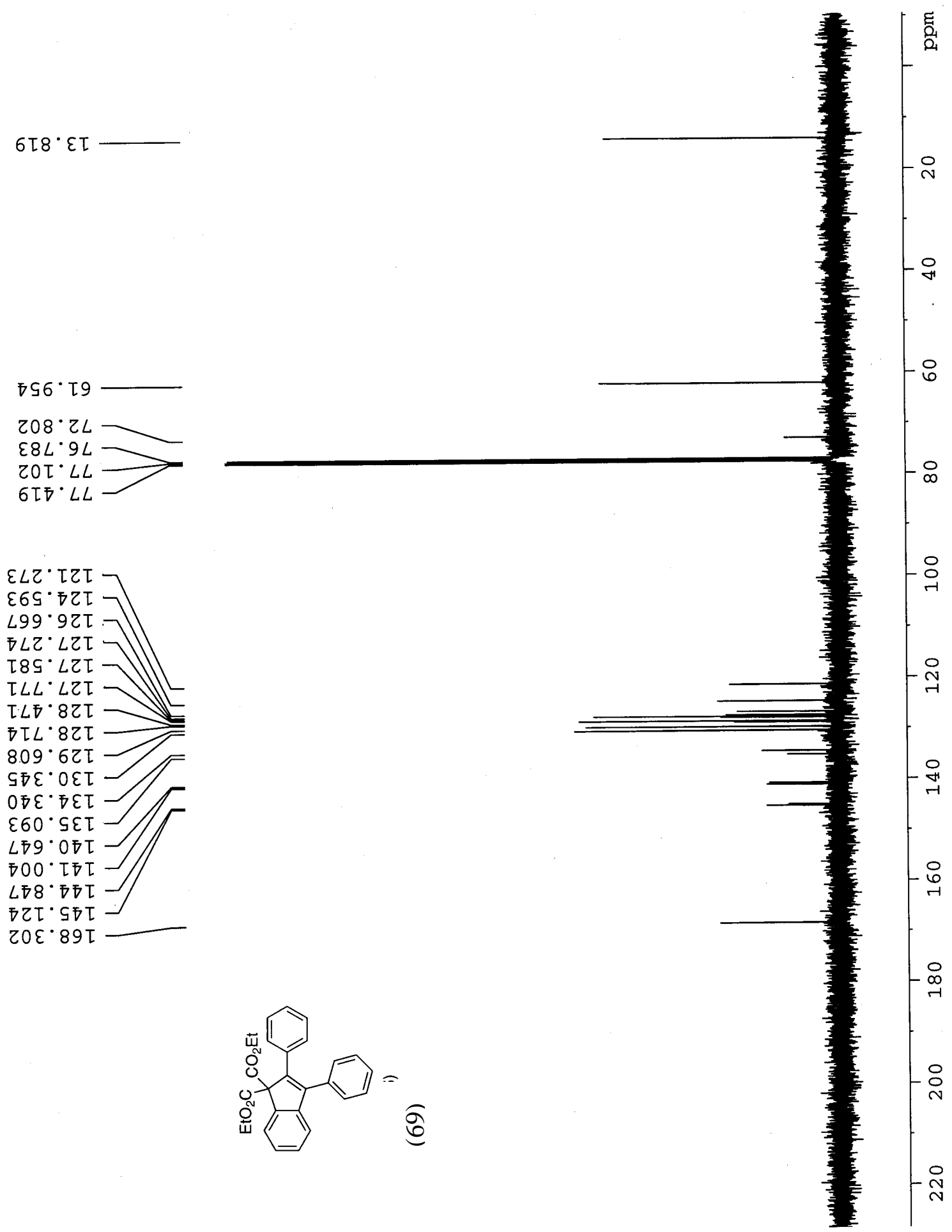


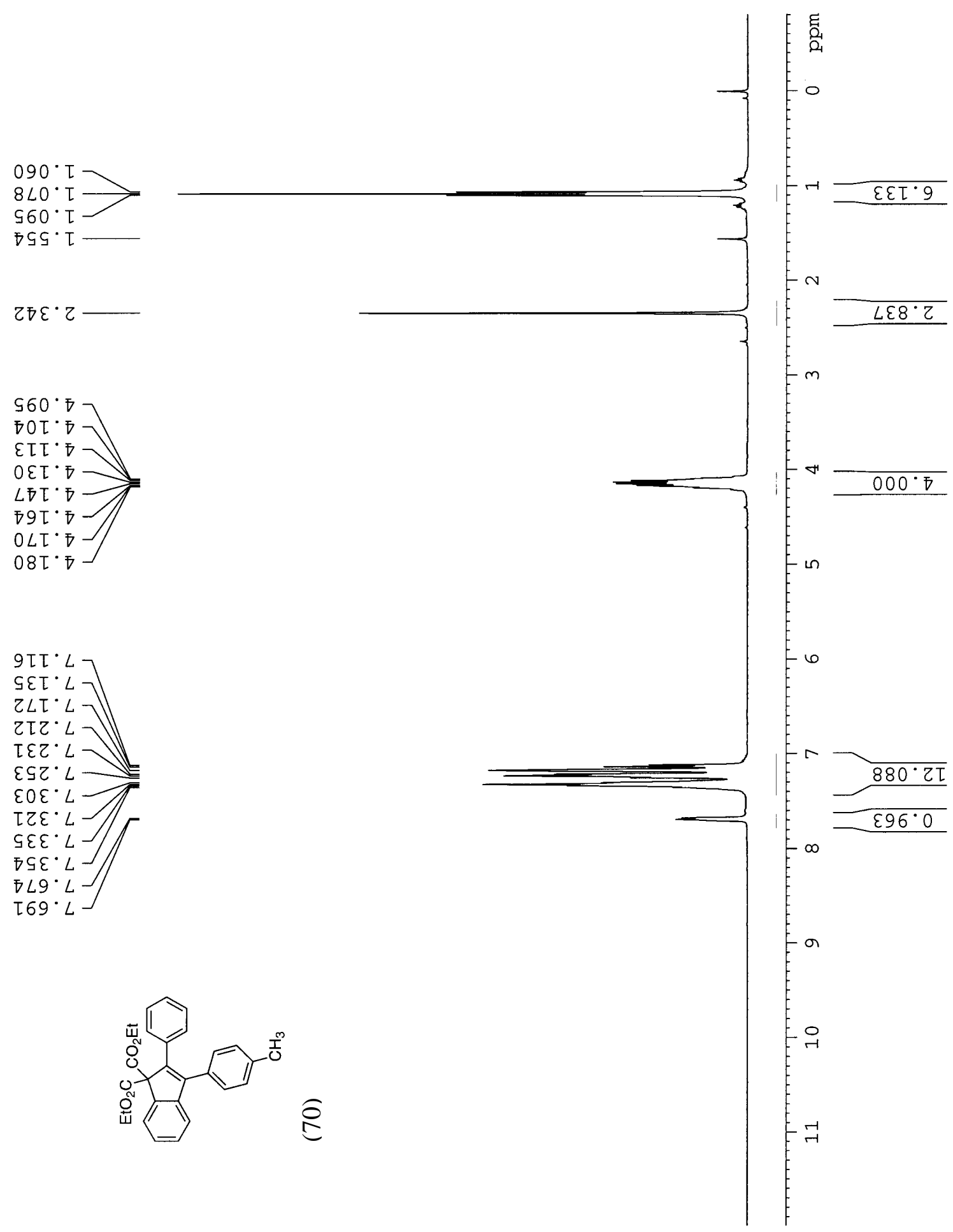


$900^{\circ}$ 万T

โ8 ${ }^{\circ}$ โZ

$\angle 60^{\circ} 29 \square$

TL8.9L

$96 \mathrm{Z}^{\circ} L L$

$6 \tau L^{\circ} L L$

与6あ・โ乙T

○ワし・テて

$69 L \cdot 9 Z$

¿LE $L Z T$

ISL $\angle Z$

$59 L^{\circ} \angle Z I$

$899^{\circ} 8 Z T$

ह५ $8^{\circ} 8 Z$

† $\angle \varepsilon^{\circ} 6 Z$

$099^{\circ} 62 \tau$

T6L $62 T$

૬ع 0 .

$8 \nabla \mathrm{S}^{\circ} 0 \varepsilon \mathrm{I}$

乙8Т. ऽEL

$099^{\circ} \angle \varepsilon T$

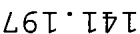

$066^{\circ}$ 呅

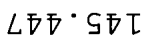

ऽऽ '89T

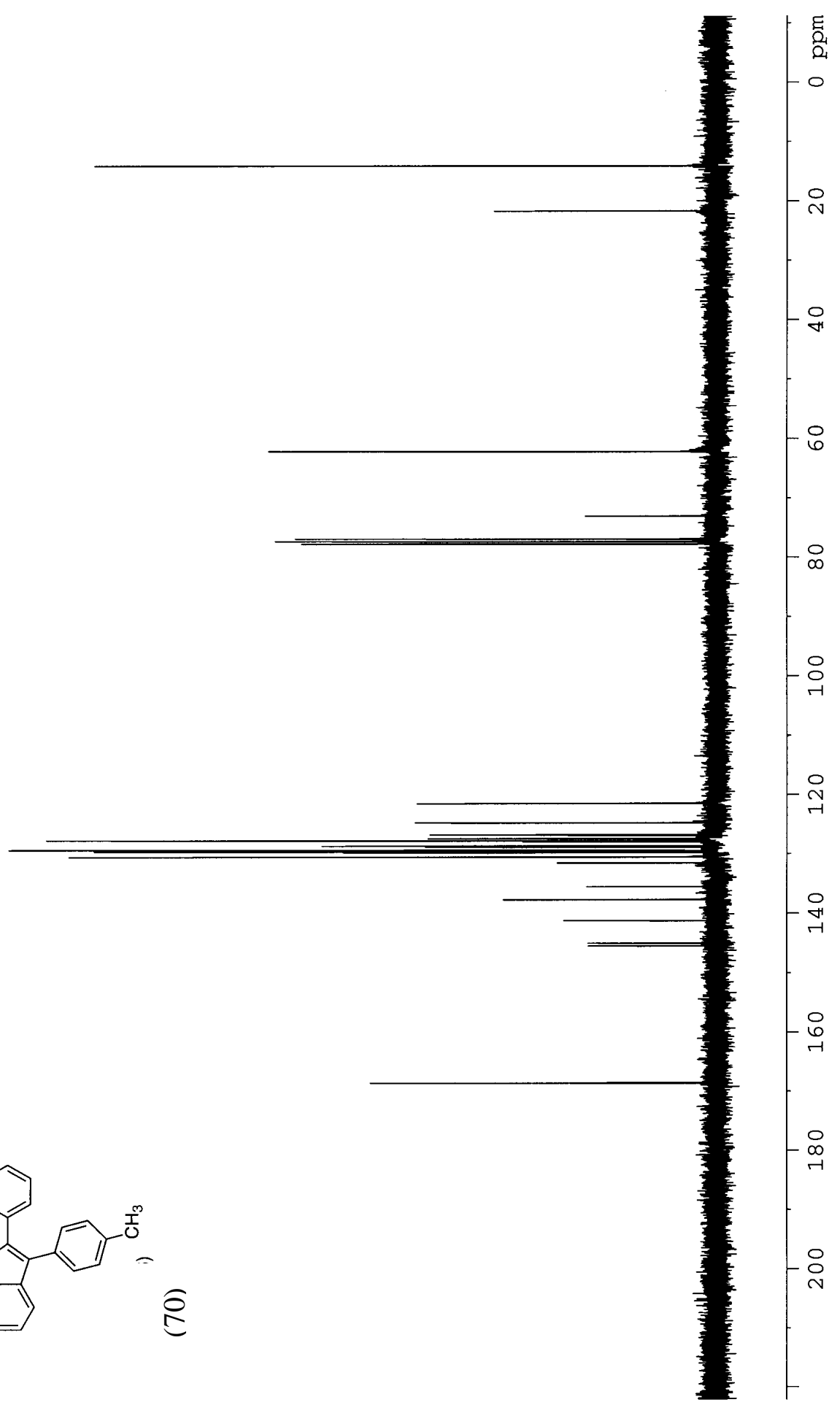




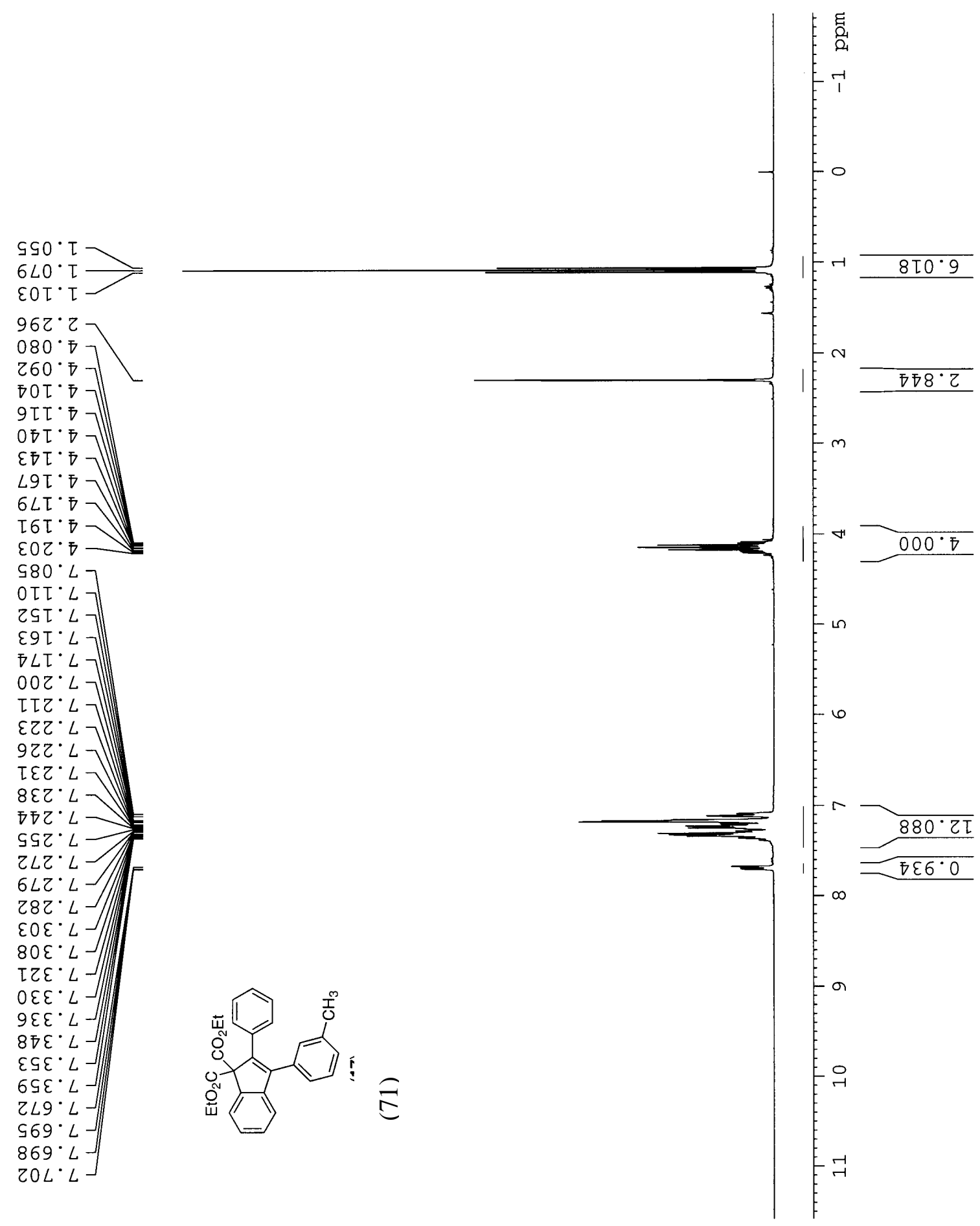



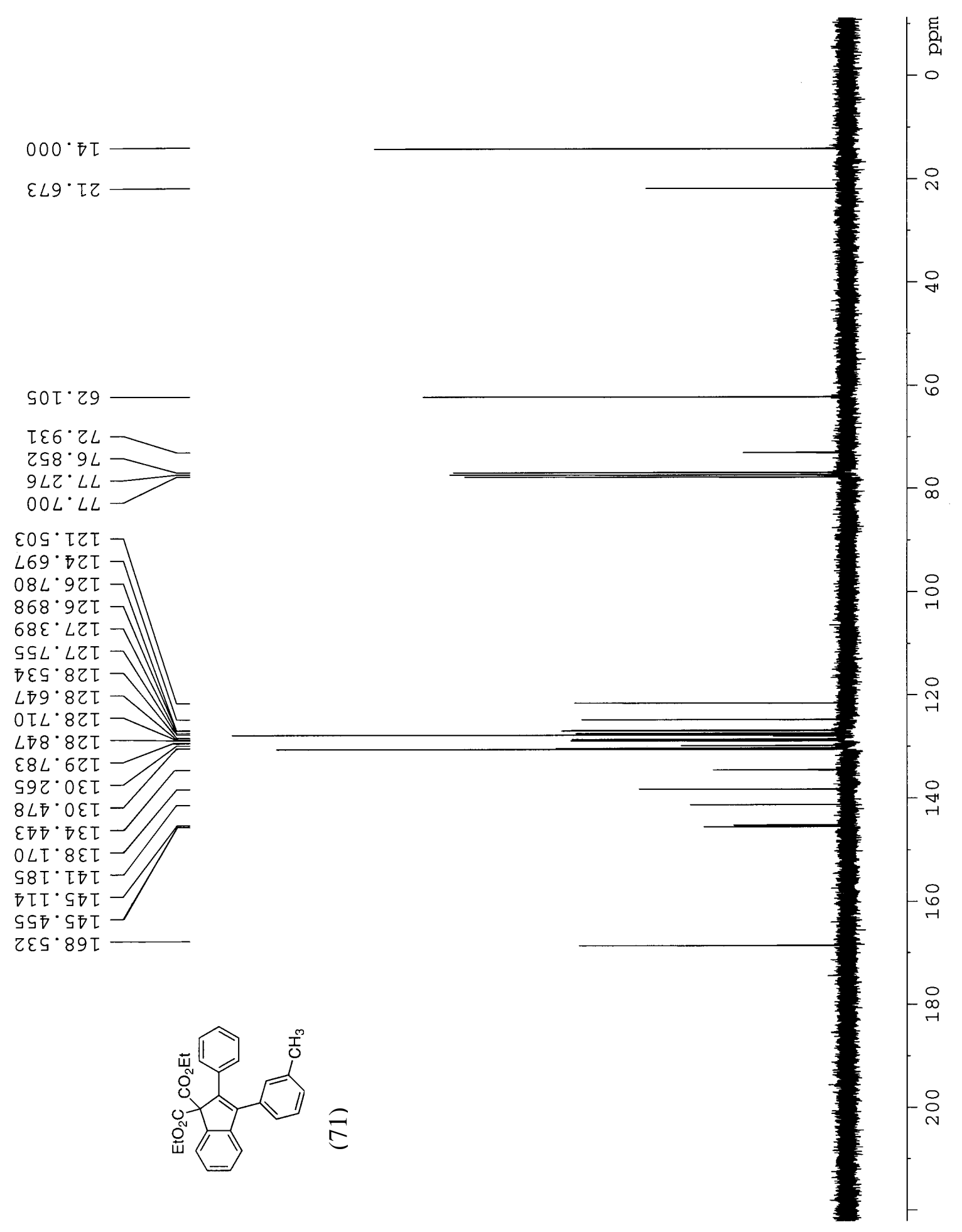


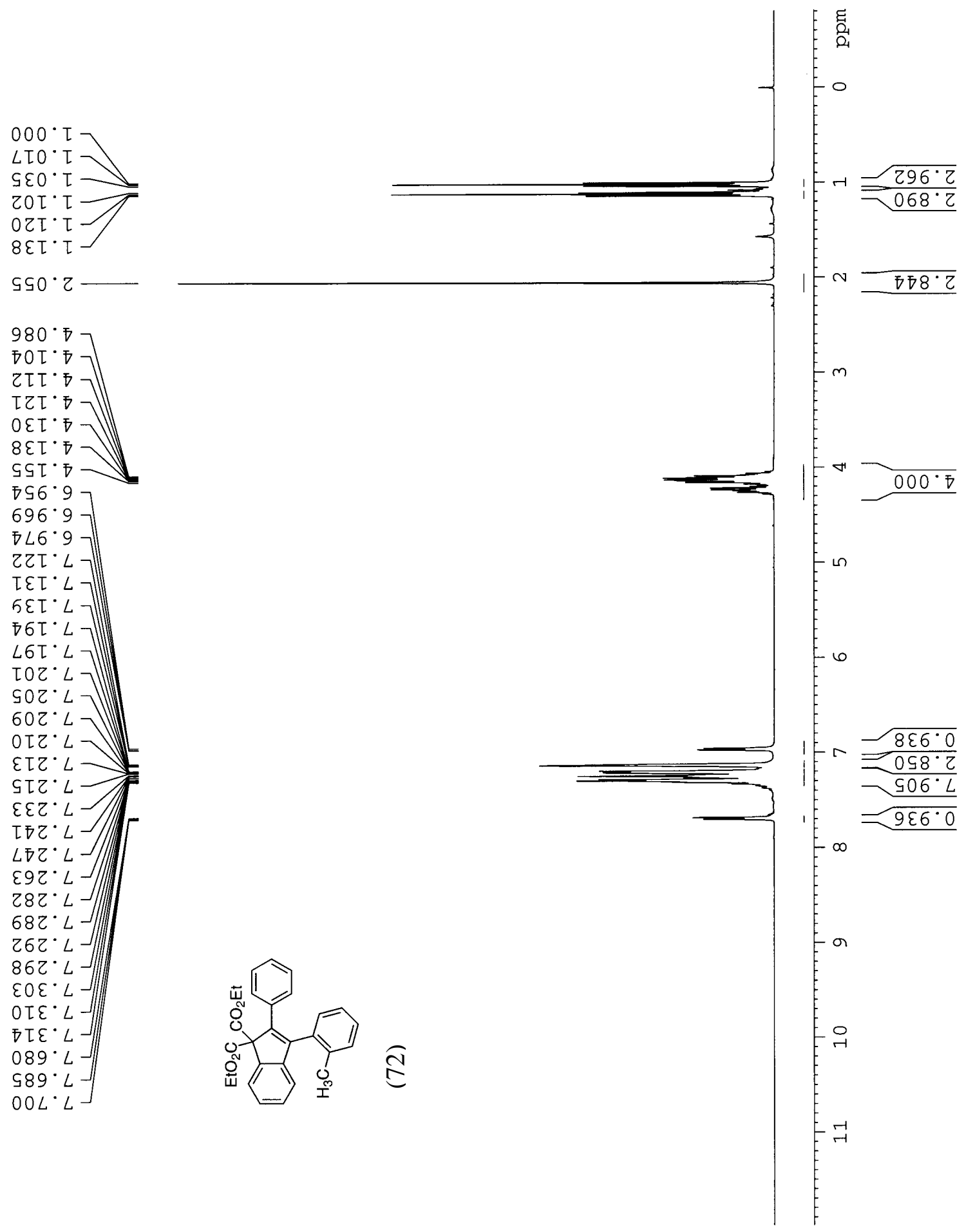


$\varepsilon \nabla L \cdot \varepsilon \tau$

$\angle L 8^{\circ} \varepsilon \tau$

$\tau \mp 9^{\circ} 6 \tau$
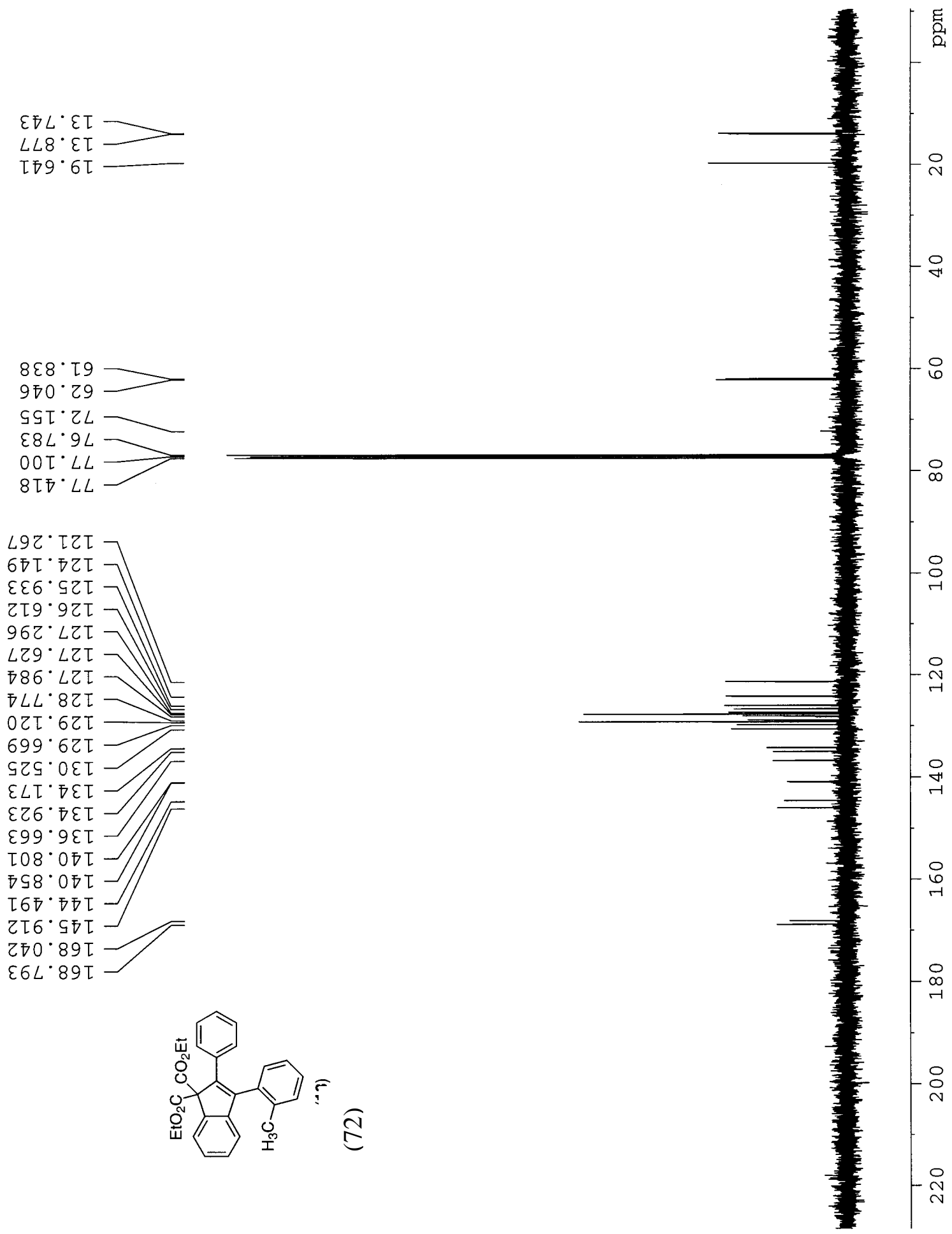


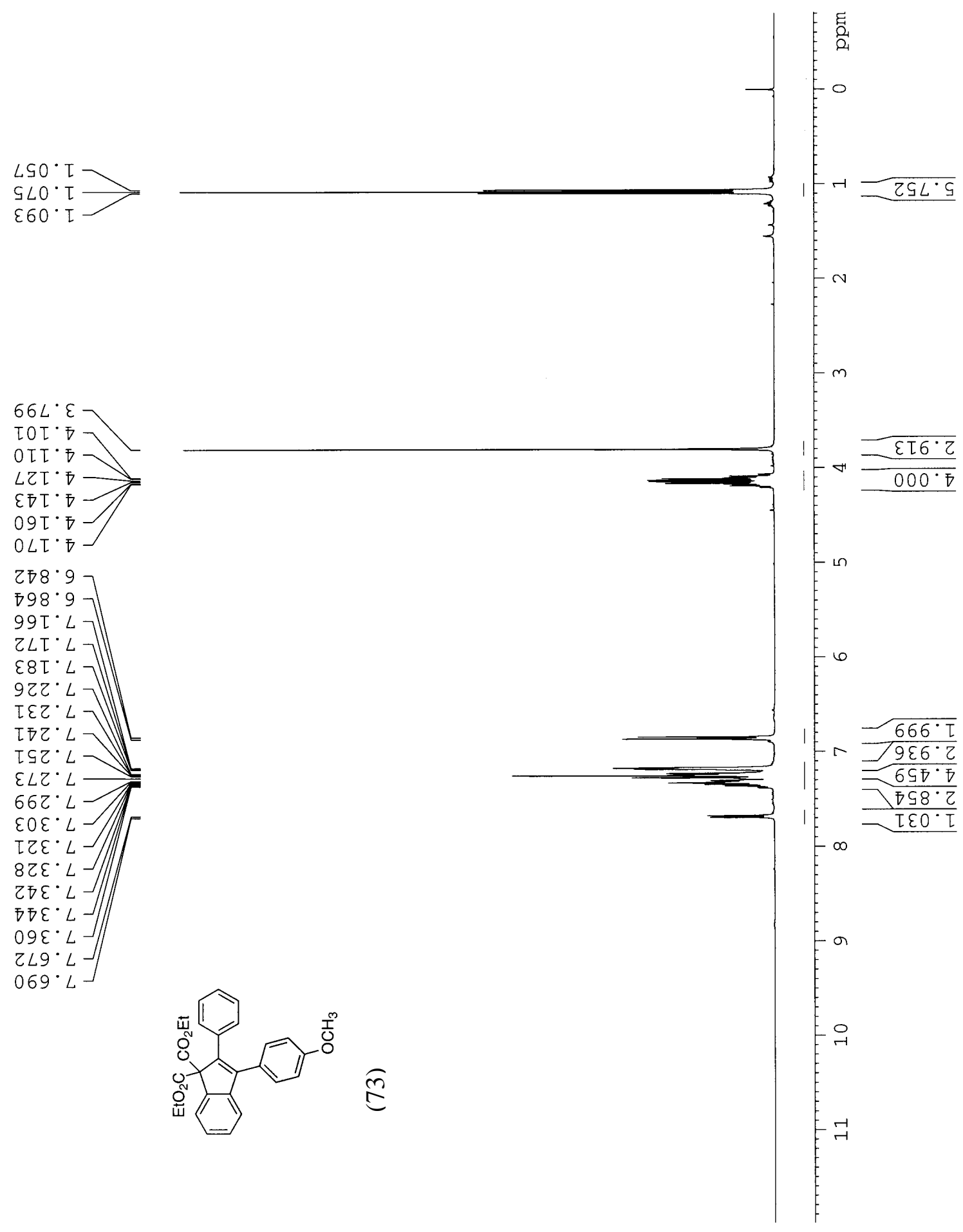




\section{$068^{\circ} \varepsilon \tau$}

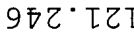

ESS'

โES.9ZI

จ9S.9ZI

$9 \varepsilon I^{\circ} \angle Z I$

$089^{\circ} \angle Z T$

ZS9.8ZT

L9 $\varepsilon^{\circ} 0 \varepsilon \tau$

०ฤ8. $0 \varepsilon \tau$

โฤ

$968 \cdot 6 \varepsilon \tau$

$6 \angle 6^{\circ} 0 \bar{T}$

โ8⿷

โ92. $5 \bar{L}$

โ80.69

乙8ह'89

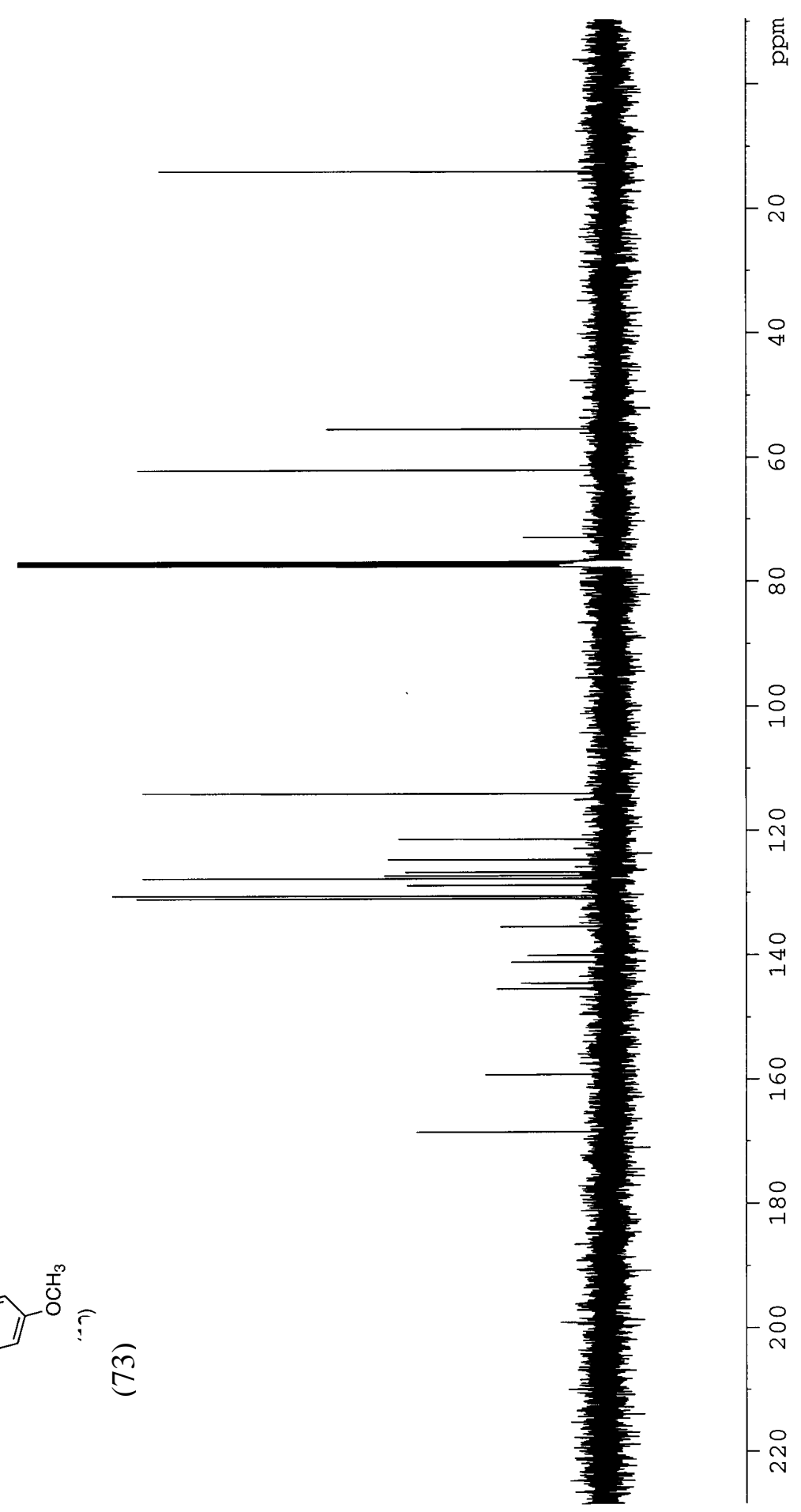




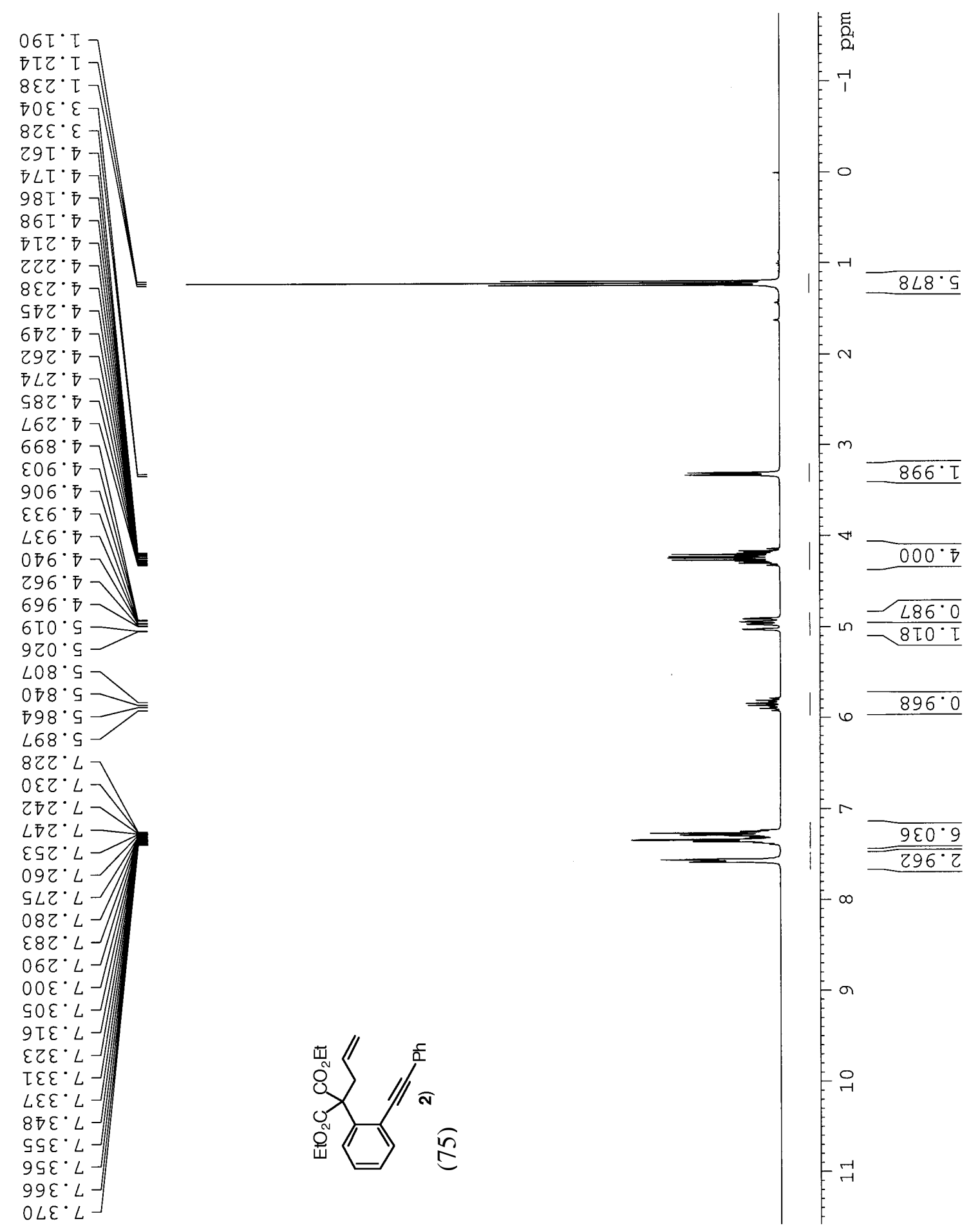




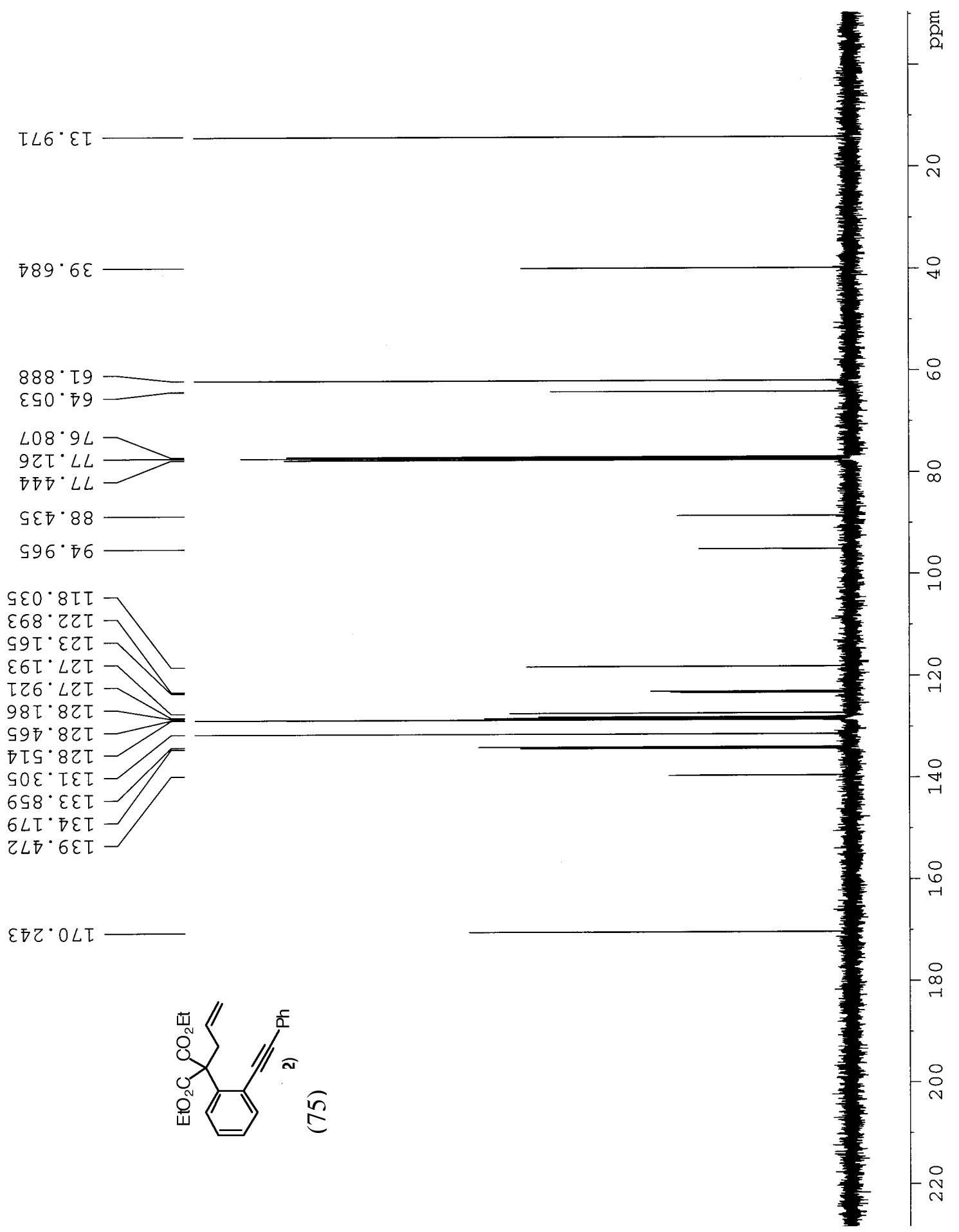




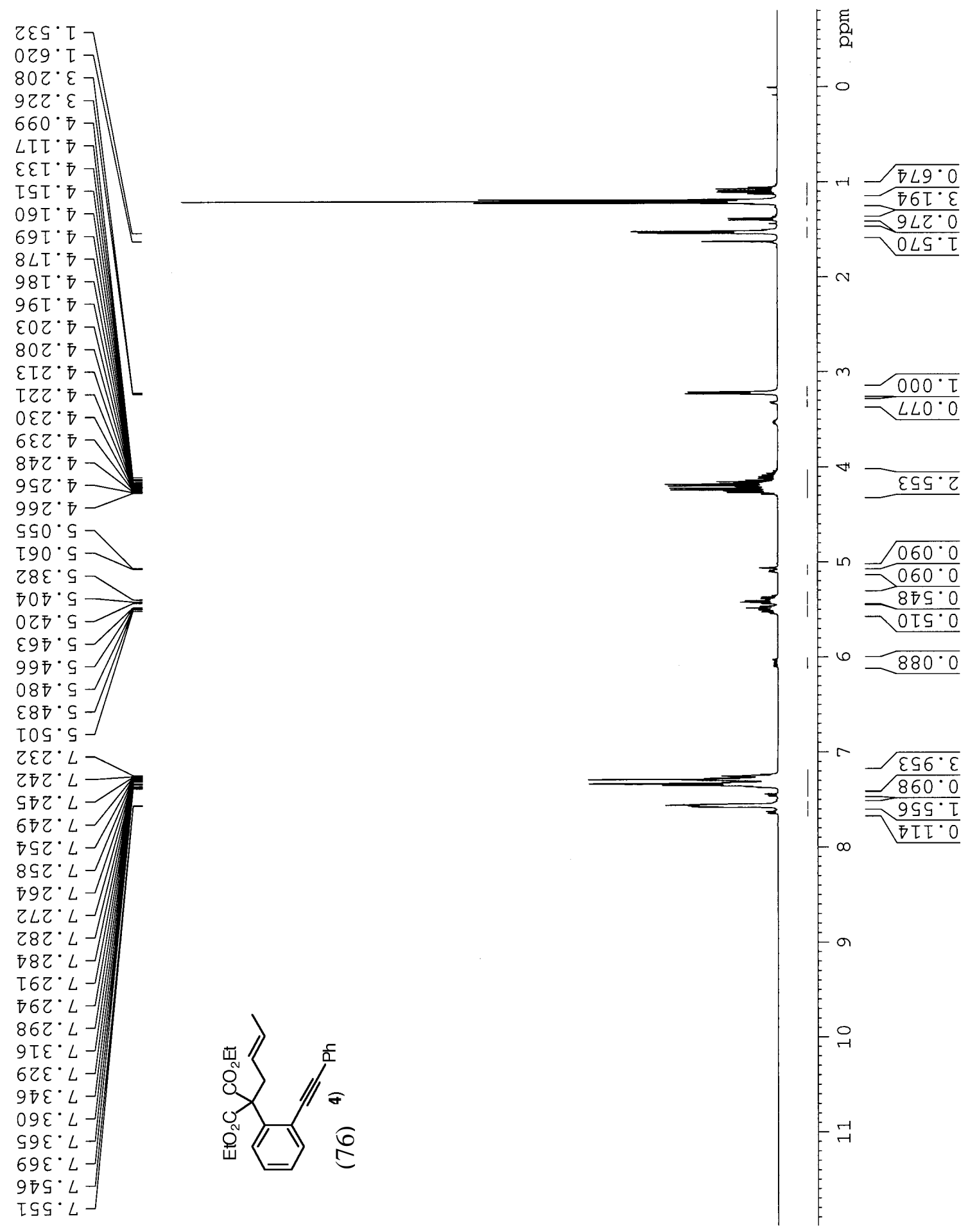


$\varepsilon\left\llcorner\sigma^{\circ} \varepsilon T\right.$

$\varepsilon 86^{\circ} L$

$โ 08 \cdot 8 \varepsilon$

SZL T9

โZZ・五

$06 L \cdot 9 L$

$\angle O T^{\circ} L L$

与乙ซ' $L L$

ELG' 88

$008^{\circ} \nabla 6$

Ђ6 $6^{\circ} \mathrm{ZT}$

乙L乙 $\varepsilon 乙 \tau$

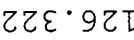

ISO $\angle Z T$

I08 $\angle Z T$

$6 \angle 8^{\circ} \angle Z T$

$90 T^{\circ} 8 Z \mathrm{~T}$

โZஏ.8ZT

$806^{\circ} 8 Z \mathrm{~T}$

Z L6 $62 \tau$

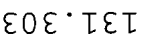

$6 L$ • $\varepsilon \varepsilon \tau$

$\varepsilon \varepsilon L^{\circ} \sigma \varepsilon \tau$

$\bar{\nabla} L Z^{\circ} O L \tau$

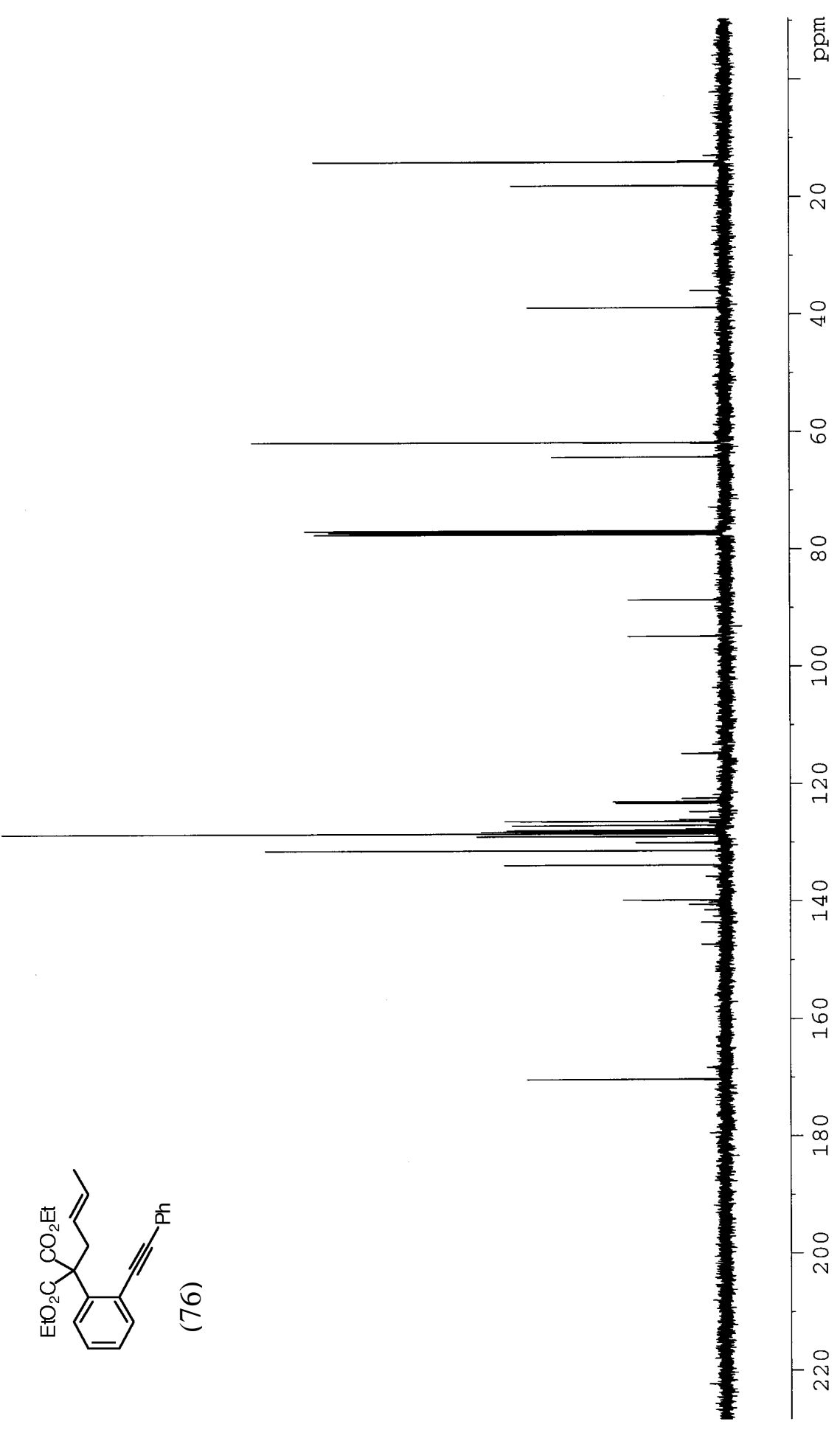




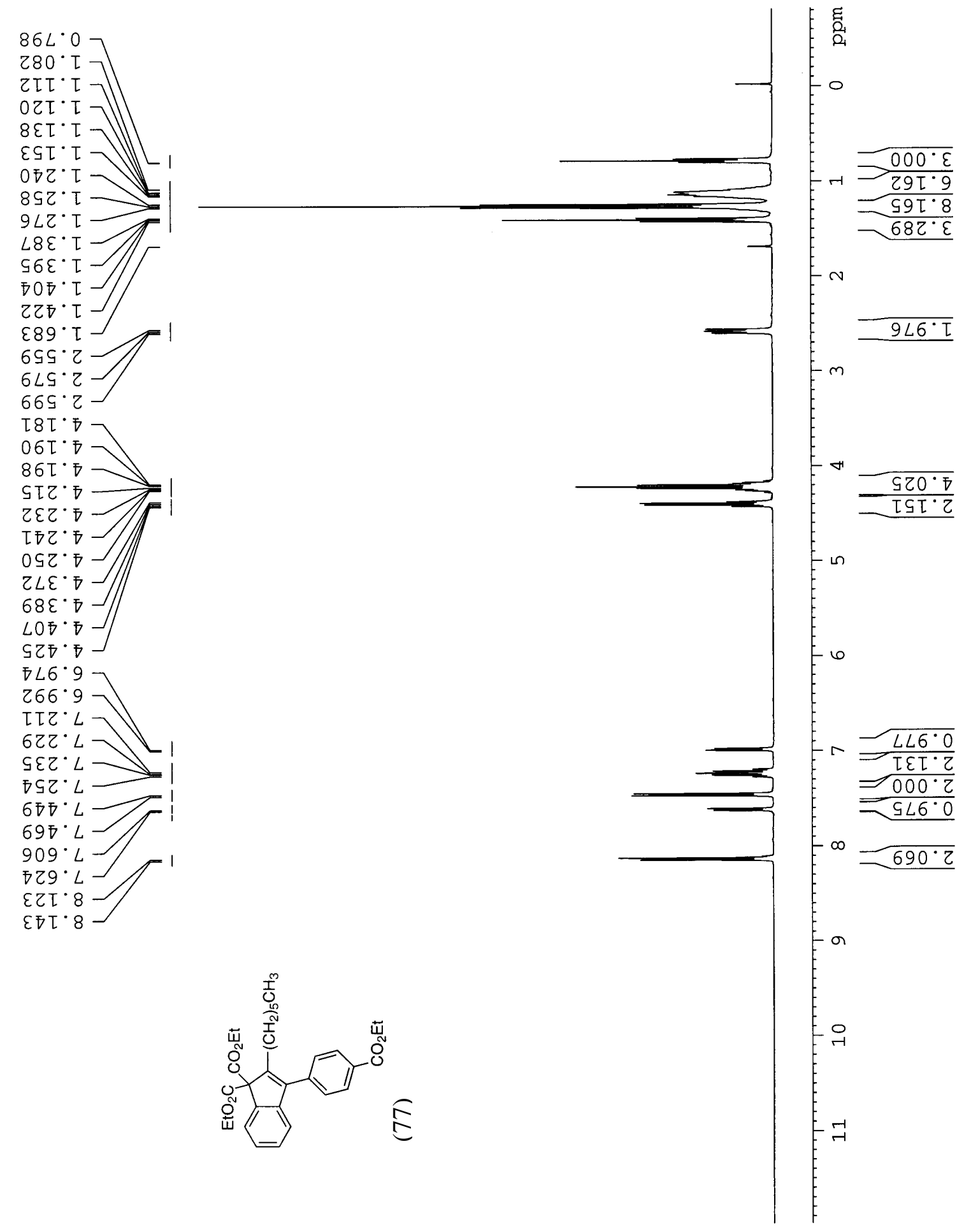


$220^{\circ} \bar{\tau}$

$6 \varepsilon 00^{\circ} \bar{\tau}$

6โ๐・元

ISG' $2 Z$

$269^{\circ} \mathrm{LZ}$

LOI' 62

万โL.6Z

$8 \tau \overline{1} \cdot \tau \varepsilon$

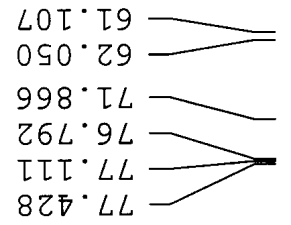

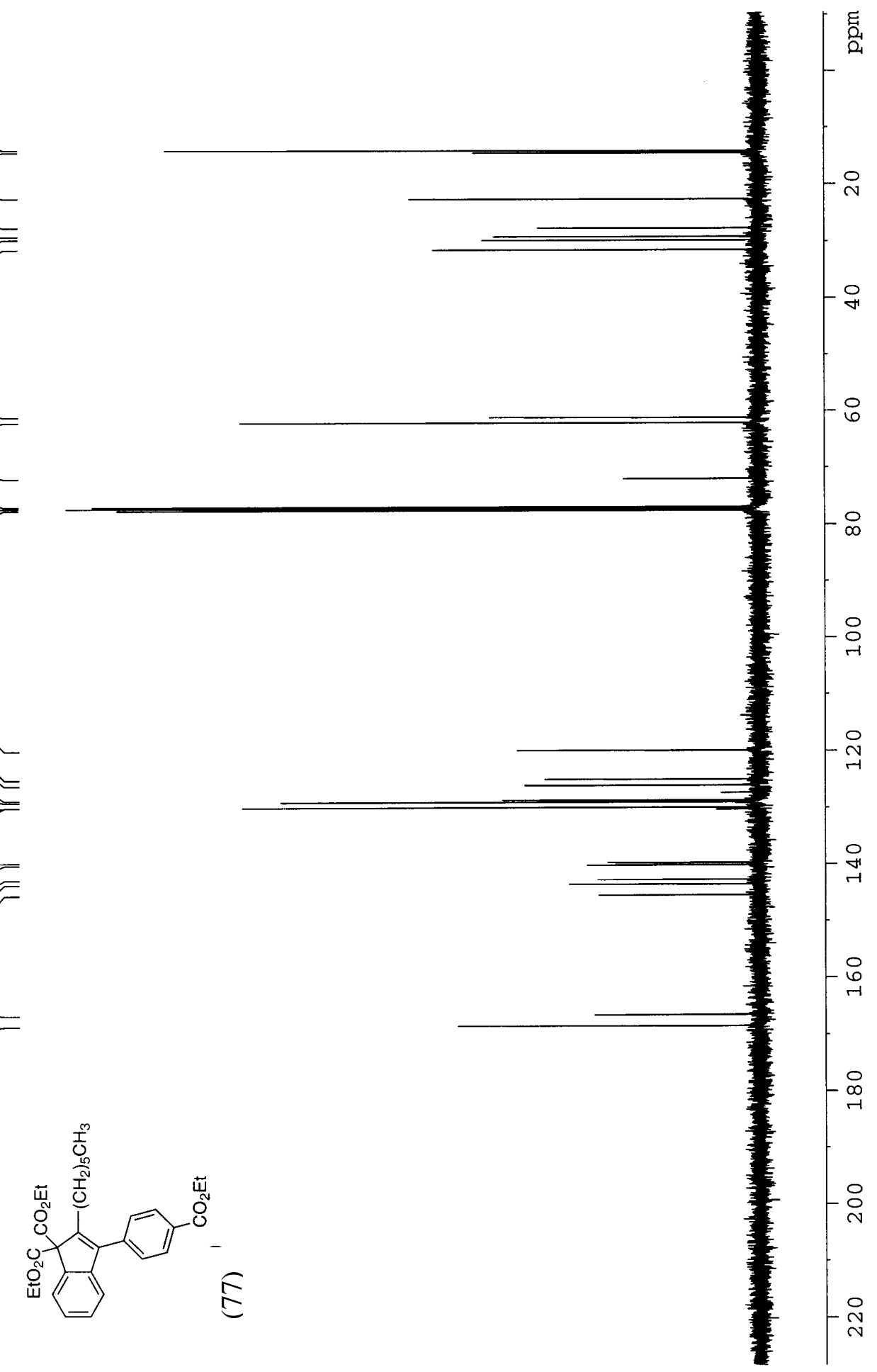

SOD. $89 \mathrm{I}$ 


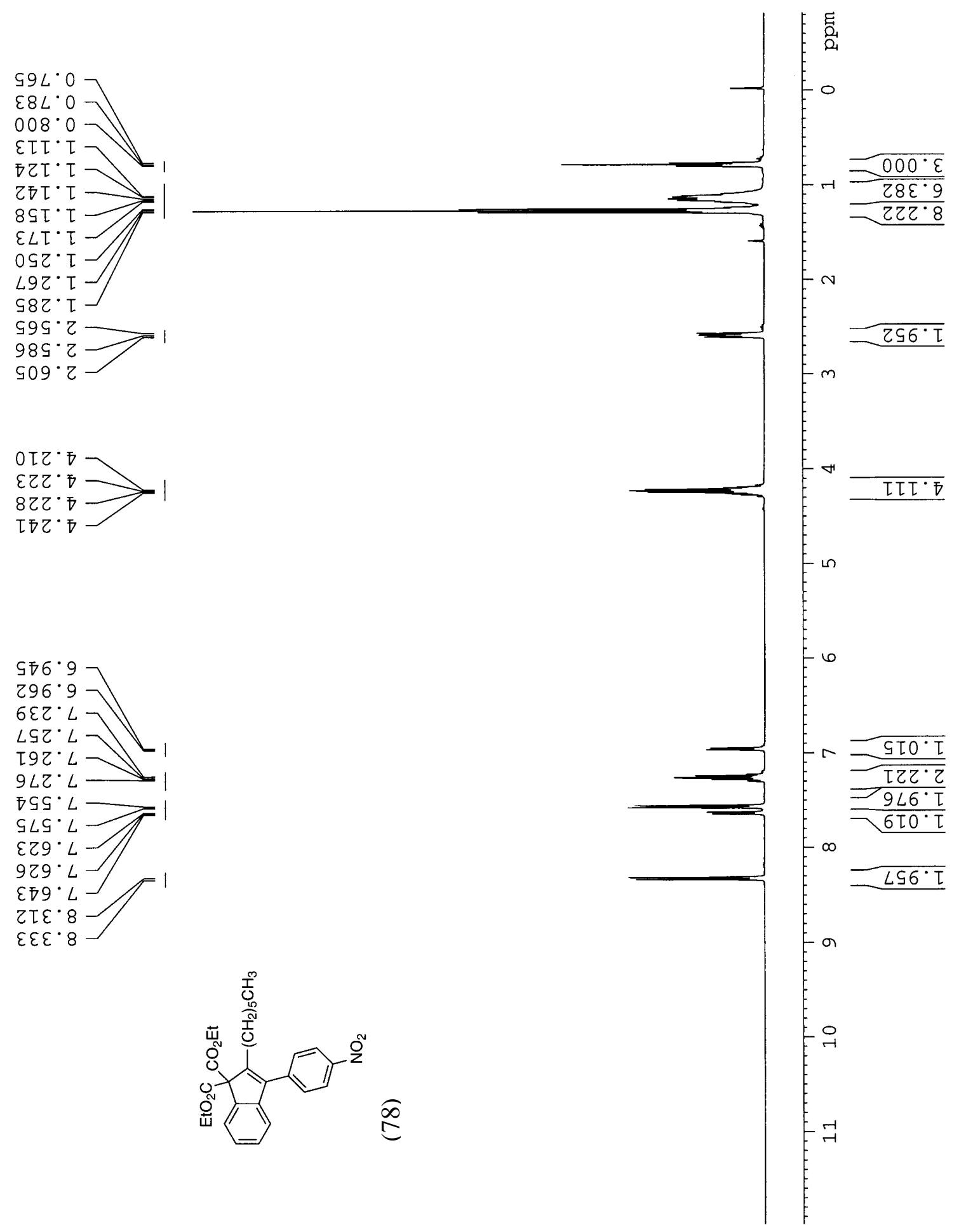


$\angle 20^{\circ} D T$

9ह5. $2 Z$

โह9. $L$

$\tau L I \cdot 6 Z$

$60 L \cdot 62$

$\varsigma 6 \varepsilon \cdot \tau \varepsilon$

sor'z9

ZL6.โL

DLL. $9 L$

$\tau 60^{\circ} \mathrm{LL}$

$60 \bar{D}^{\circ} \angle L$

$509^{\circ} 6 \mathrm{~T}$

$\angle 86^{\circ} \varepsilon \tau T$

乙てह・9乙I

$90 L \cdot 8 Z T$

与โ6.6ZT

$\tau 66^{\circ} 6 \varepsilon \tau$

Z05. โ๐T

9โ6. IีT

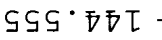

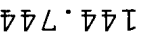

OOD $\angle \nabla T$

$90 I \cdot 89 I$

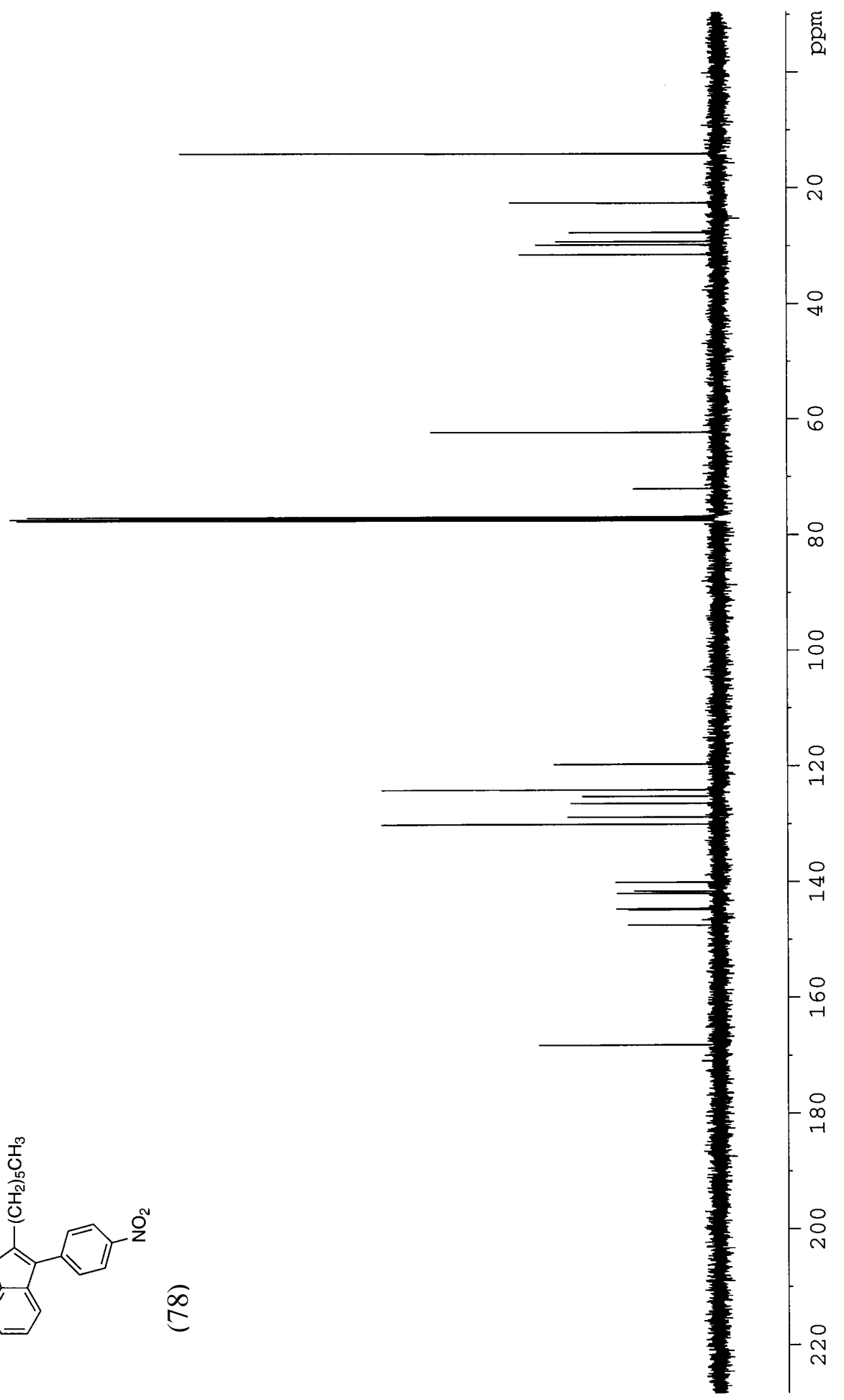




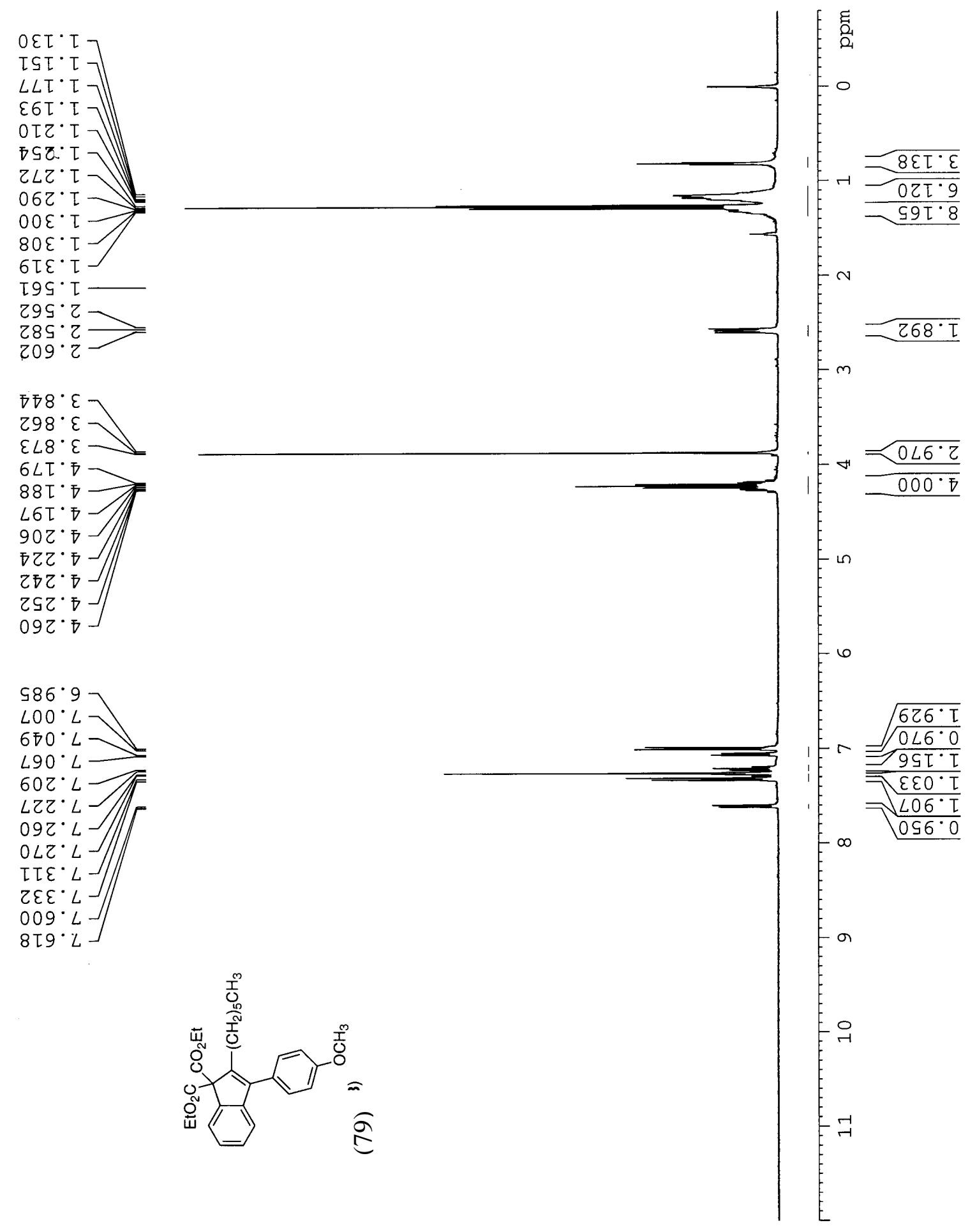




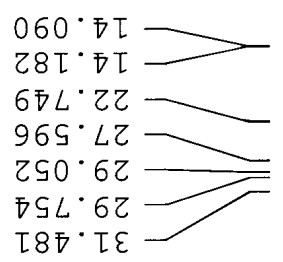

५حદ' ऽ

$806^{\circ}$ โ9

$8 \pi 9^{\circ} \mathrm{TL}$

$9 \nabla L \cdot 9 L$

$\varepsilon 90^{\circ} L L$

$\tau 8 \varepsilon^{\circ} L L$

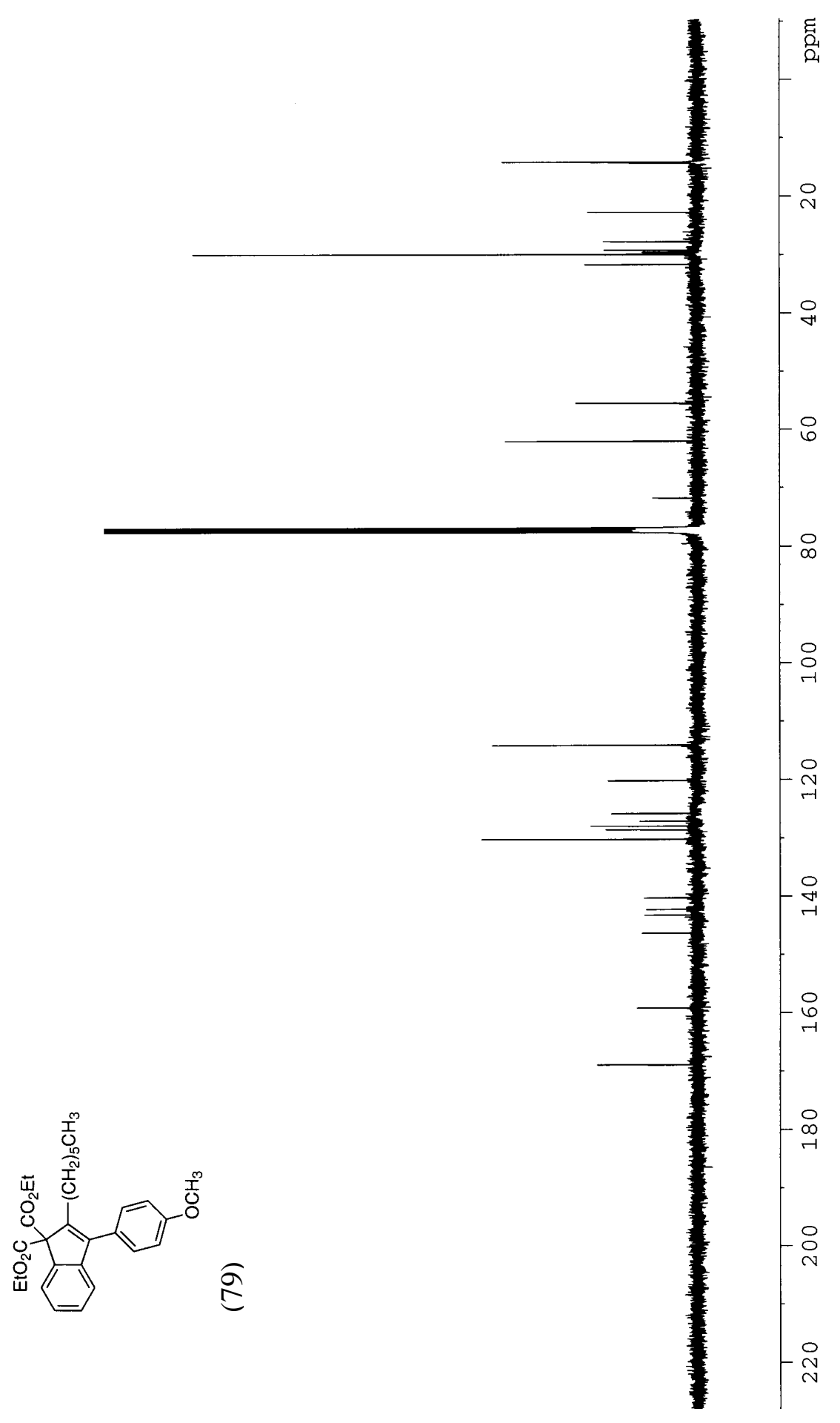

$66 \tau \cdot \nabla \tau I$

$700^{\circ} 0 z$

$929^{\circ} \varsigma Z \tau$

$8 \varepsilon 6^{\circ} 9 z$

$\varepsilon 8 L^{\circ} L Z I$

乙โォ.8ZT

ऽ๐०००દI

$00 \tau \cdot 0 \bar{T}$

$280^{\circ}$ ZTI

$890^{\circ} \varepsilon \bar{\tau} \mathrm{T}$

$\angle 9 T \cdot 9 \nabla T$

$890.69 \mathrm{~T}$ 

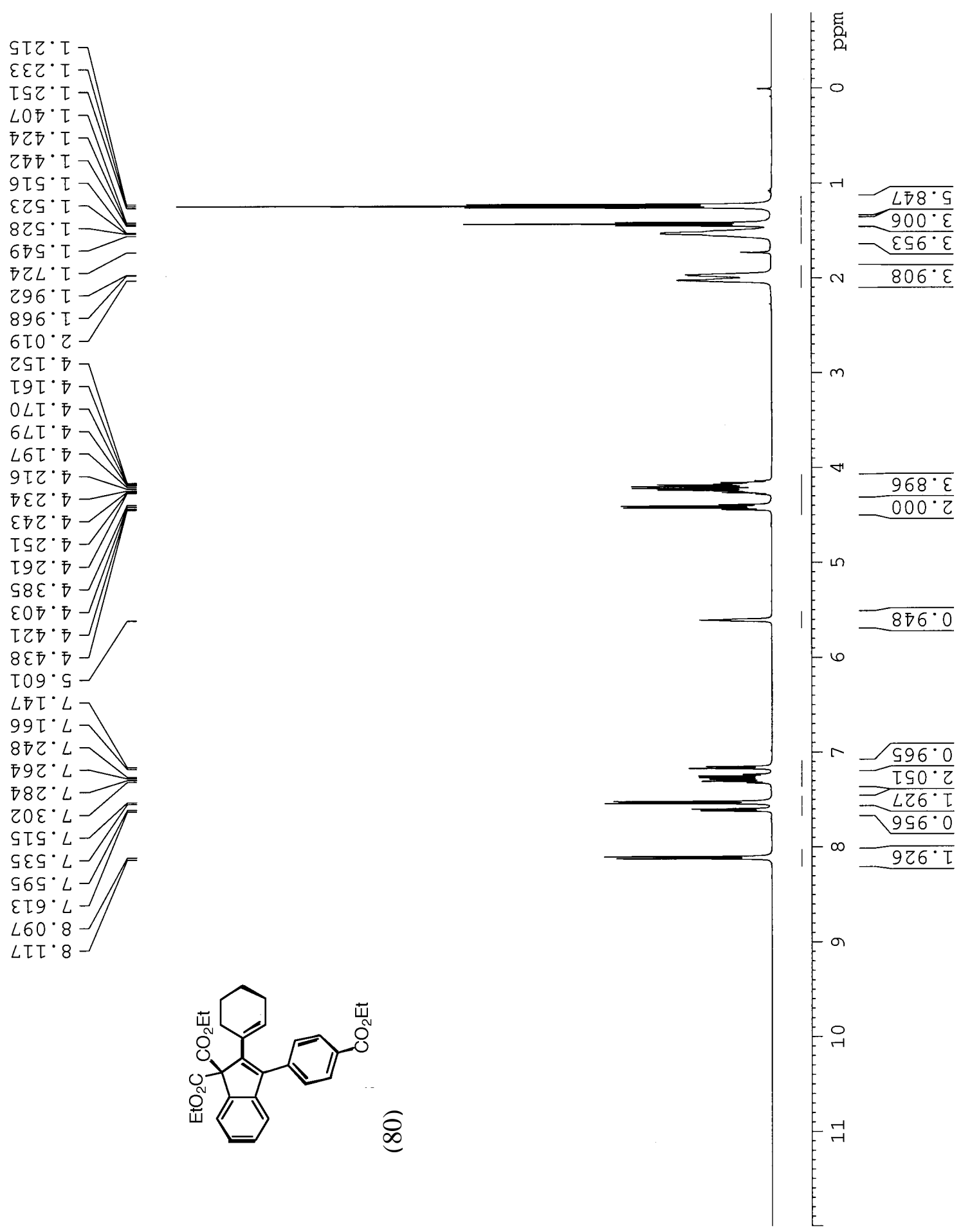


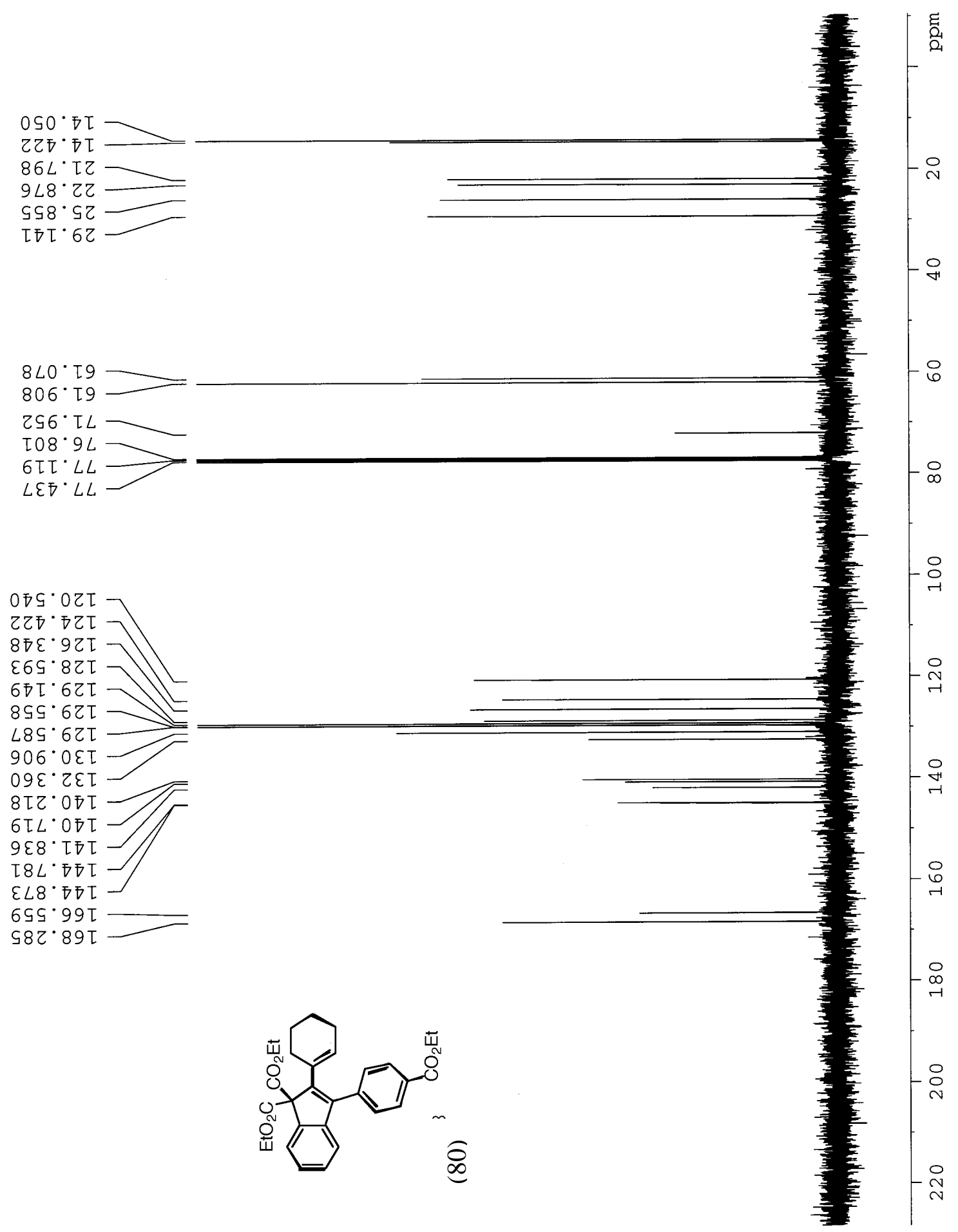




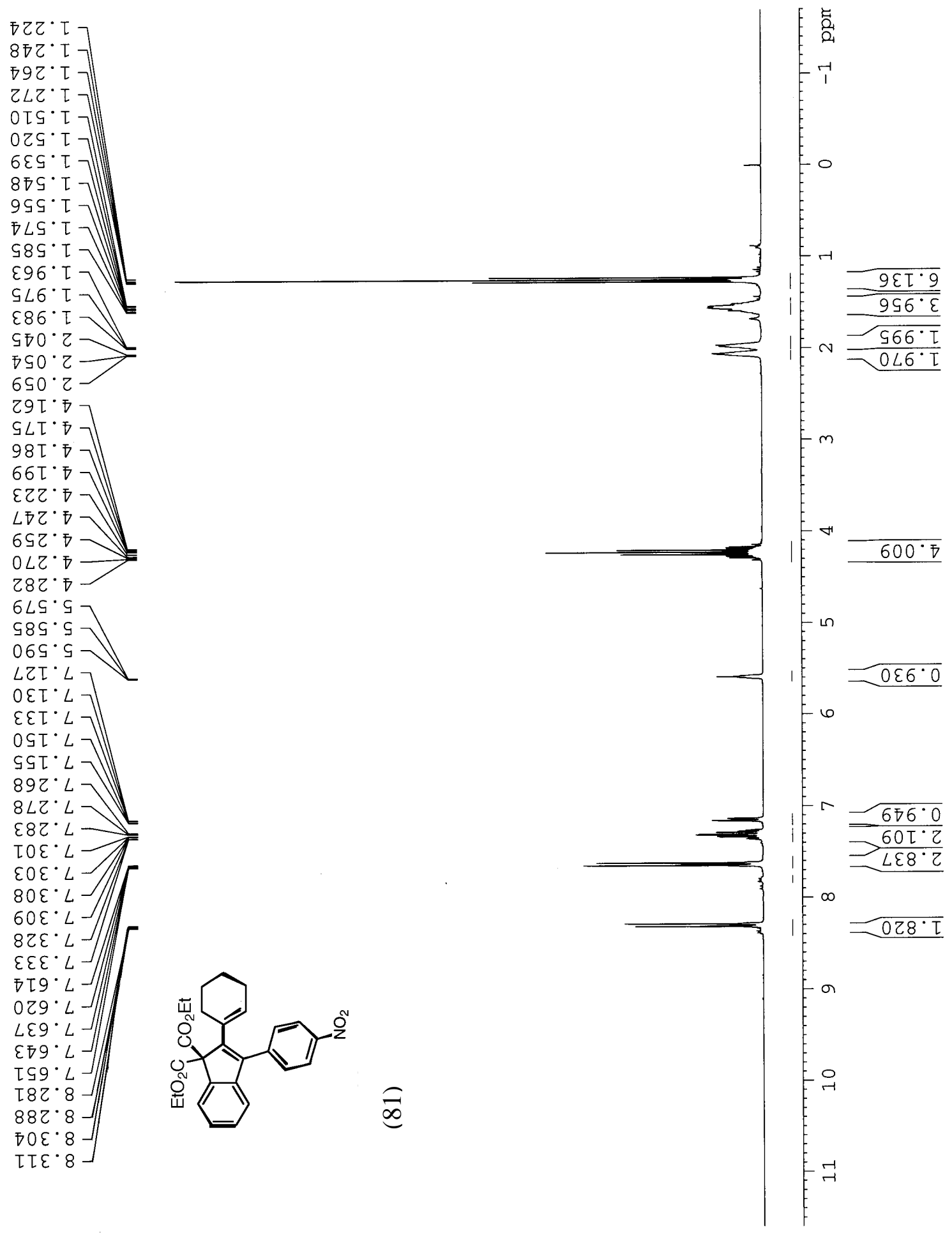


$9 \varepsilon Z^{\circ} \bar{\tau}$

$606^{\circ}$ TZ

$986^{\circ} \mathrm{ZZ}$

ฤธ0.9Z

乙५ 6 . 62

8ъて・29

$6 \varepsilon \tau \cdot \tau L$

$8 L 8^{\circ} 9 L$

$\varepsilon O \varepsilon^{\circ} L L$

$L Z L^{\circ} L L$

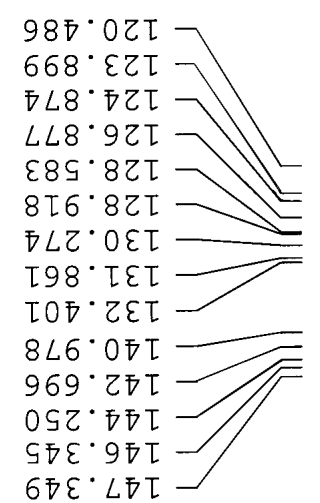

$97 \tau \cdot 89 \tau$

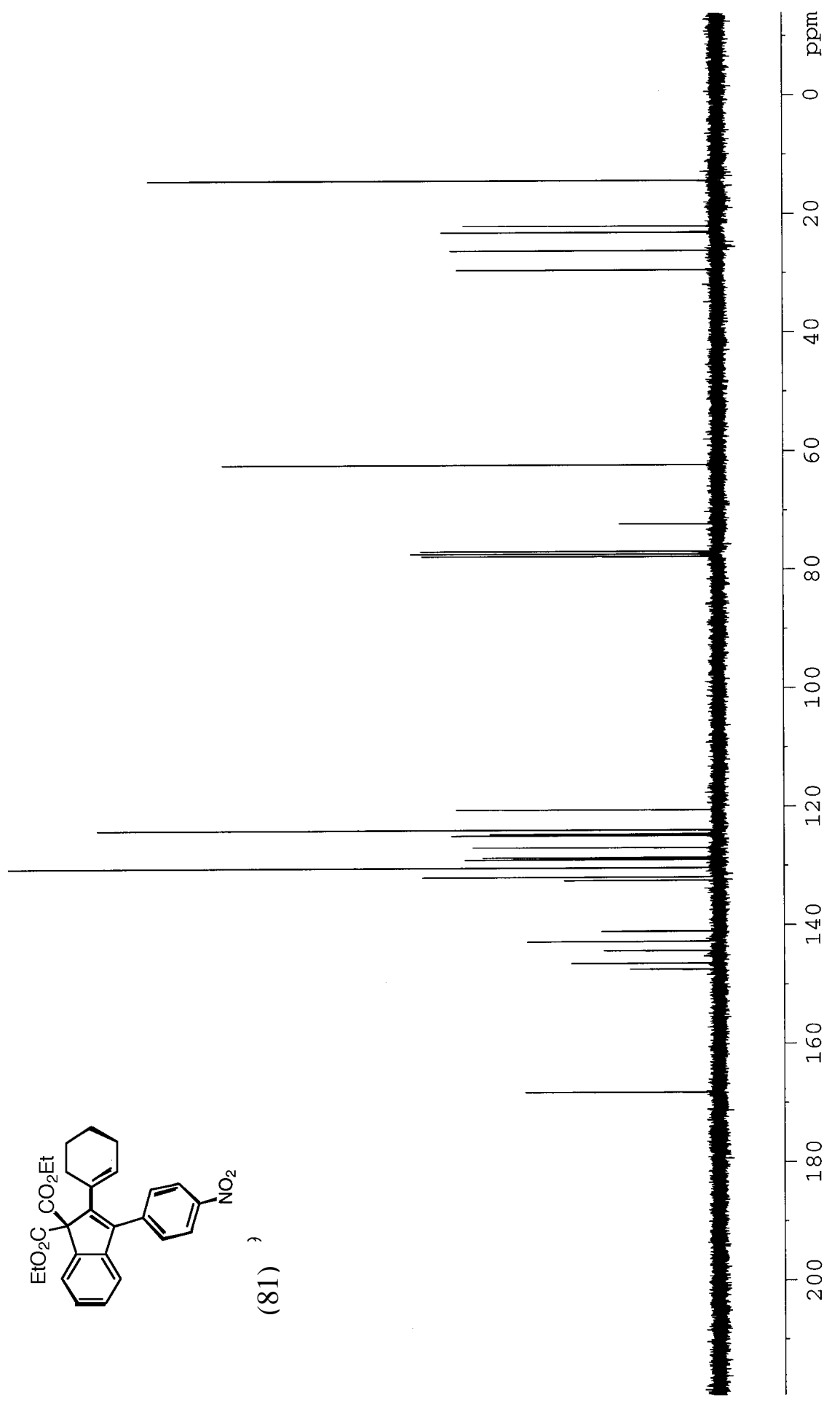




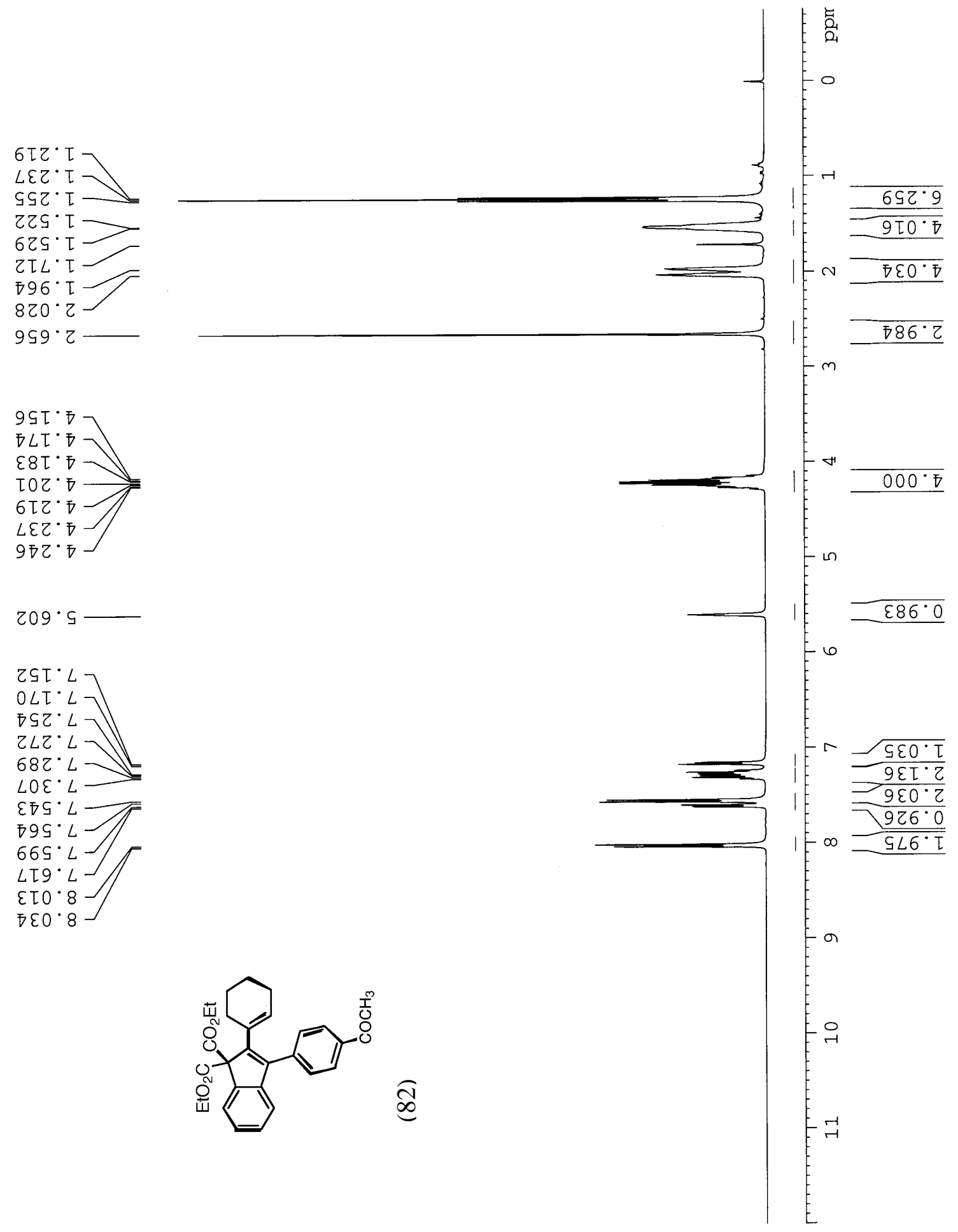




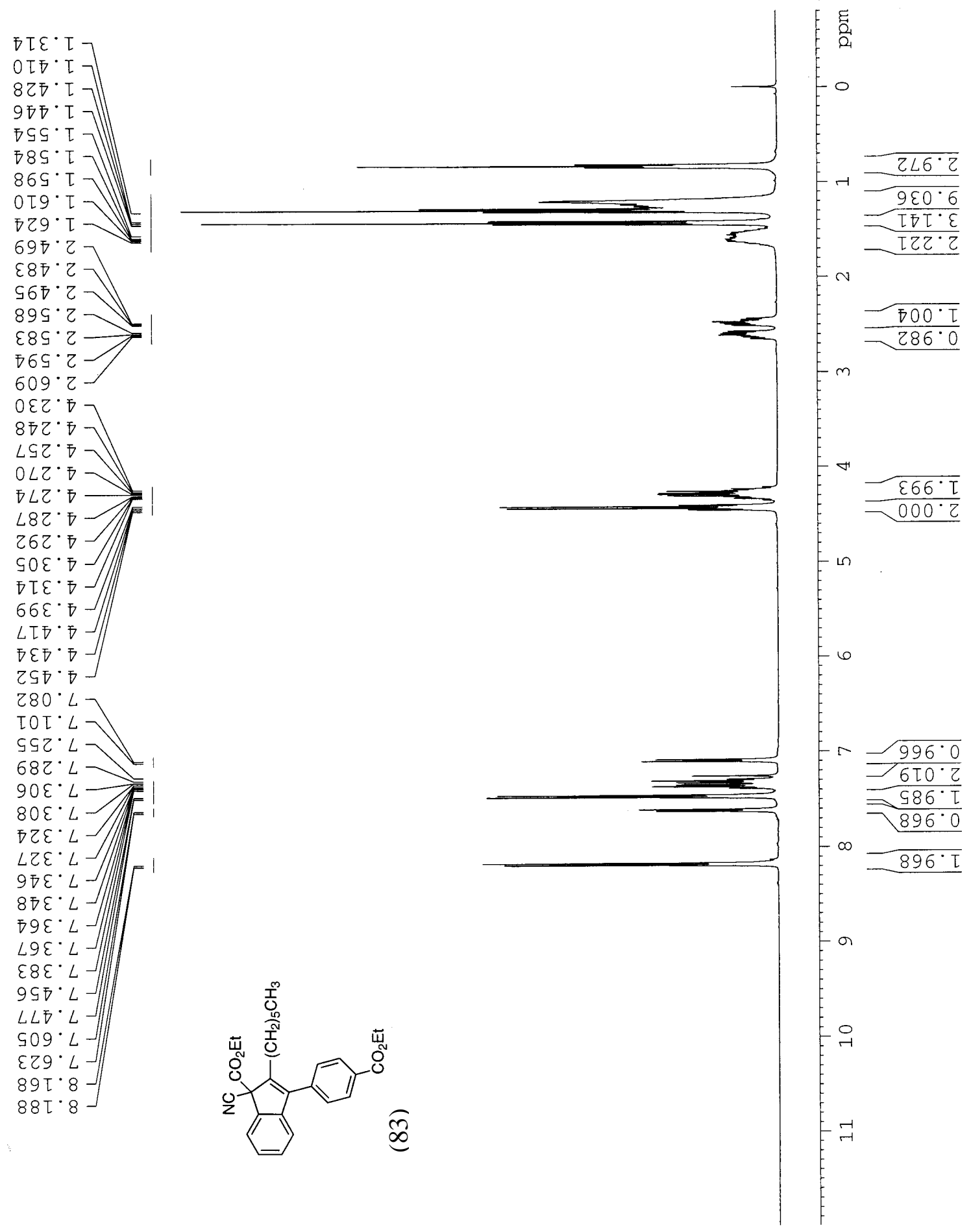




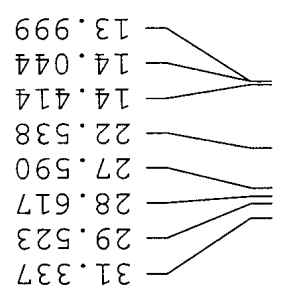

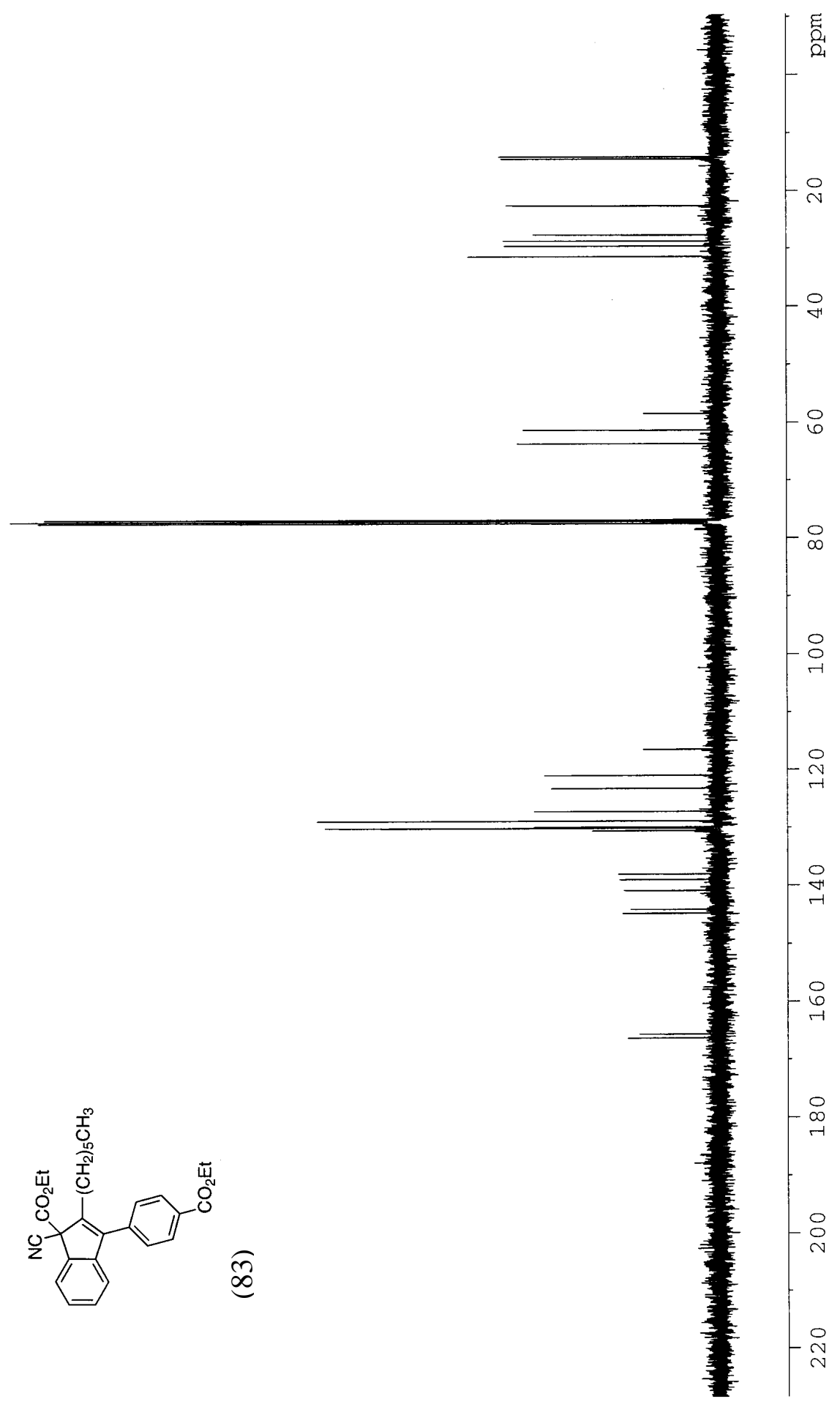
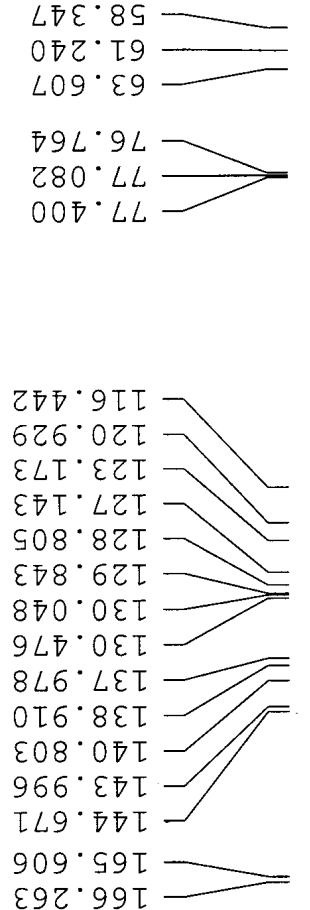

$000^{\circ} \angle L L$

$\varepsilon 97 \cdot 99 \mathrm{I}$ 


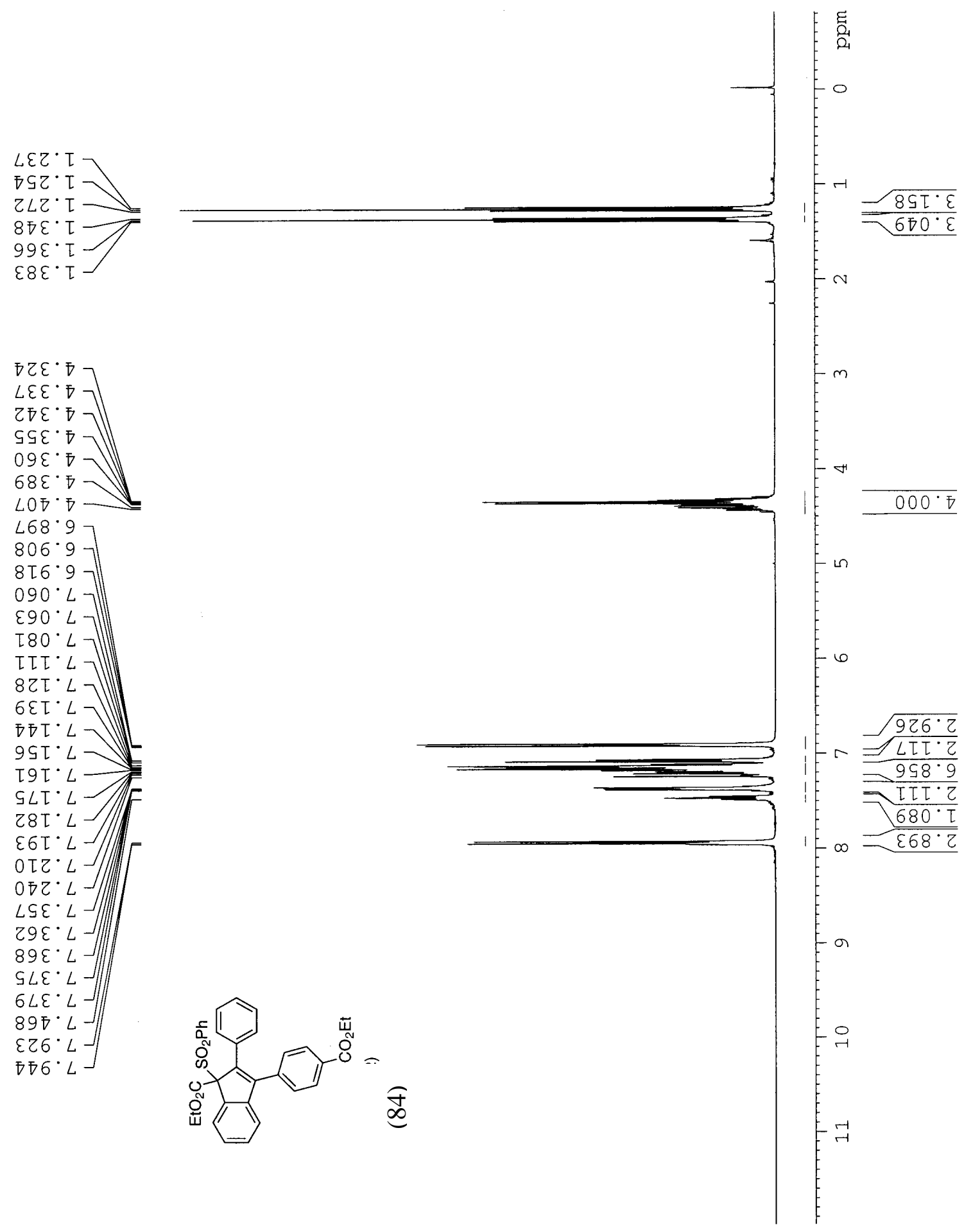


$L 26^{\circ} \varepsilon \tau \longrightarrow$

$9 L \varepsilon \cdot \nabla T \longrightarrow$
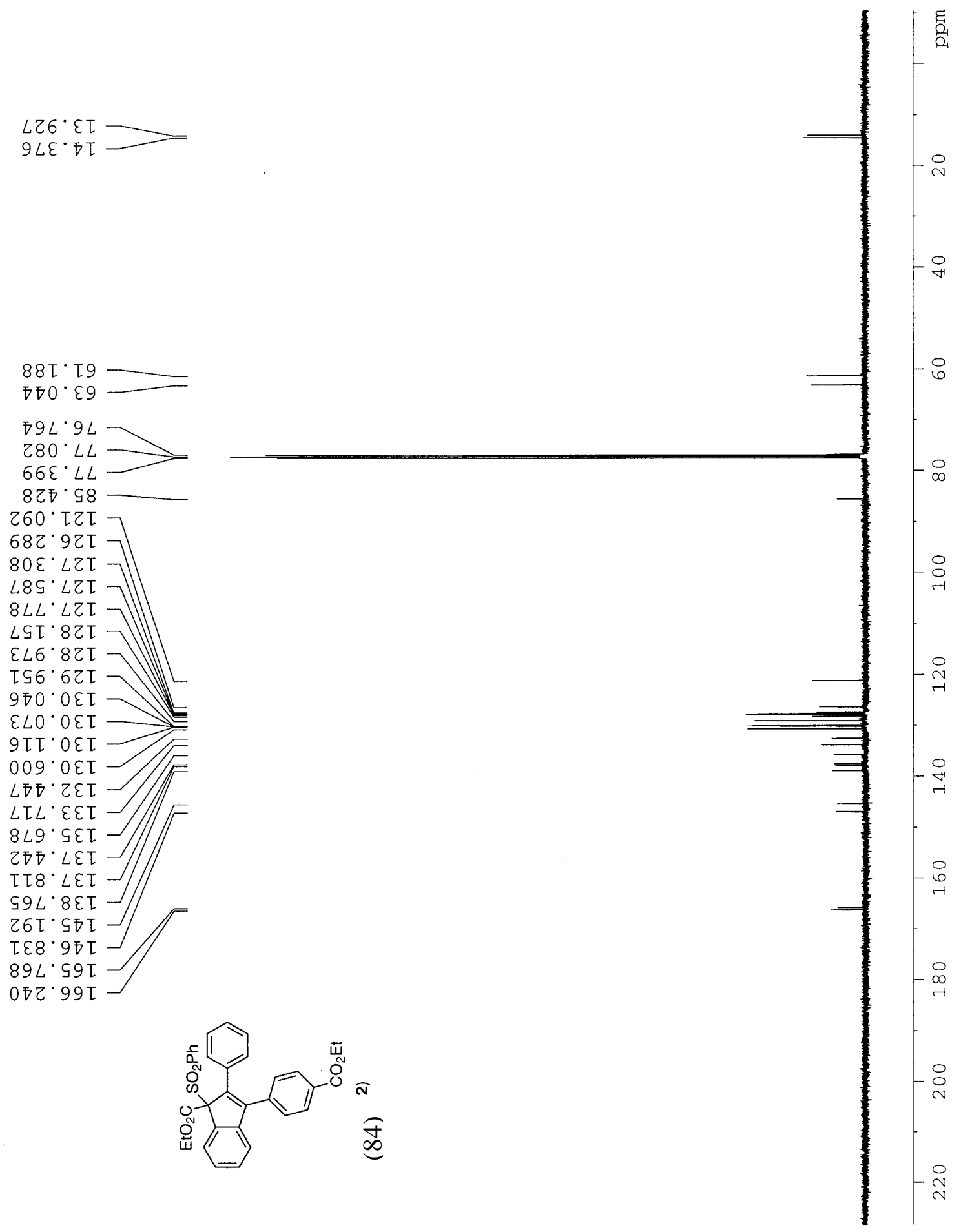


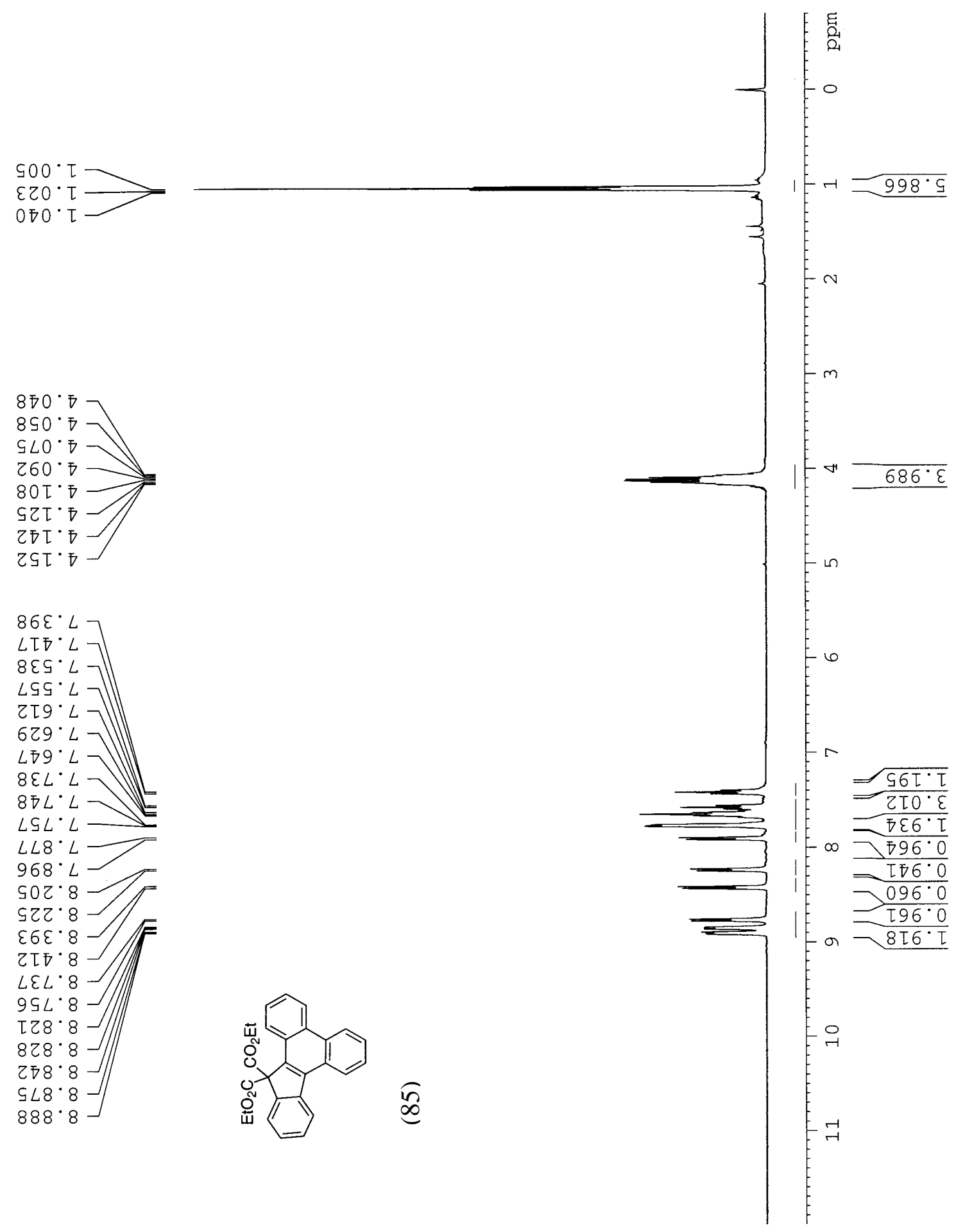




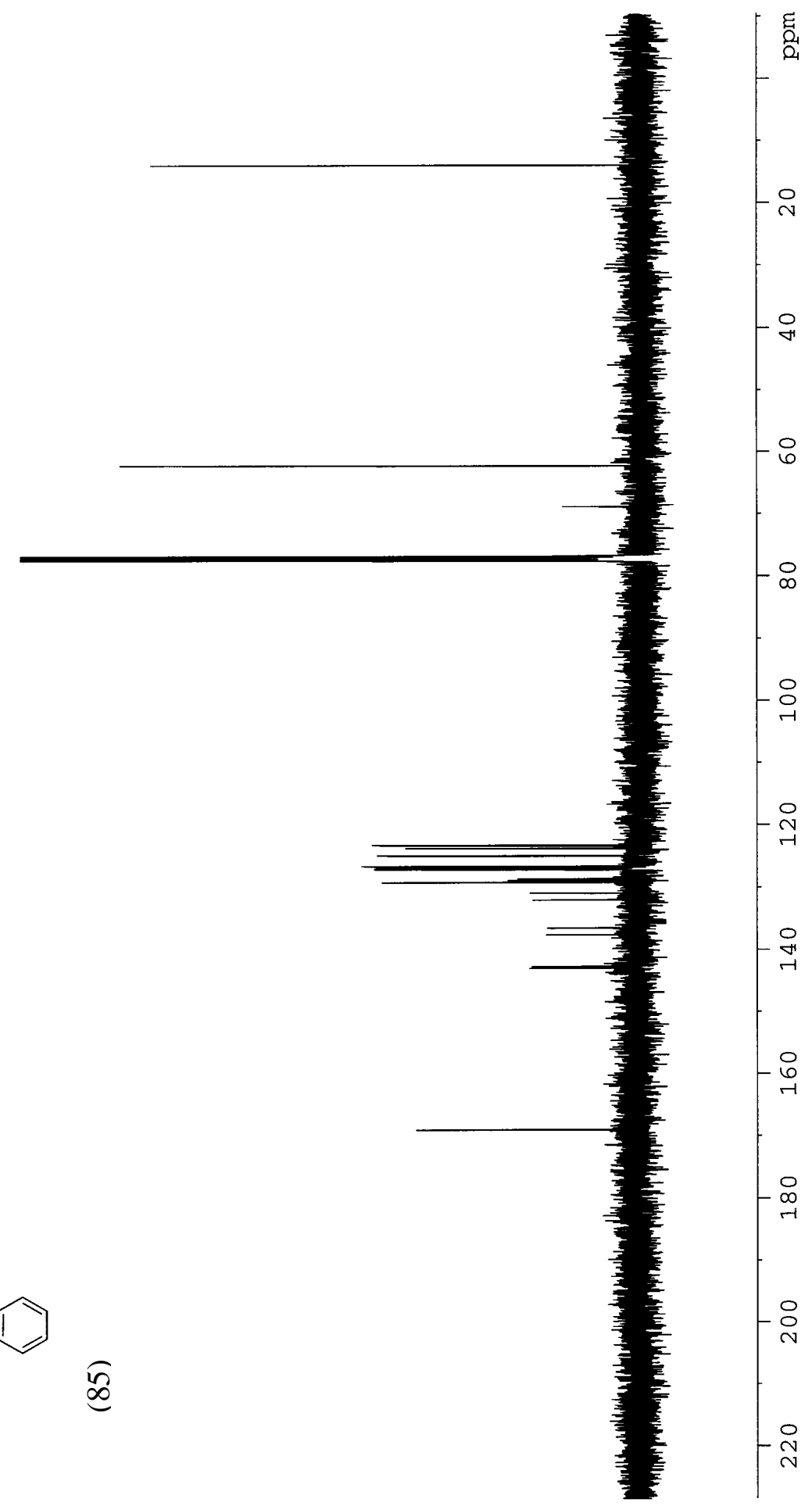




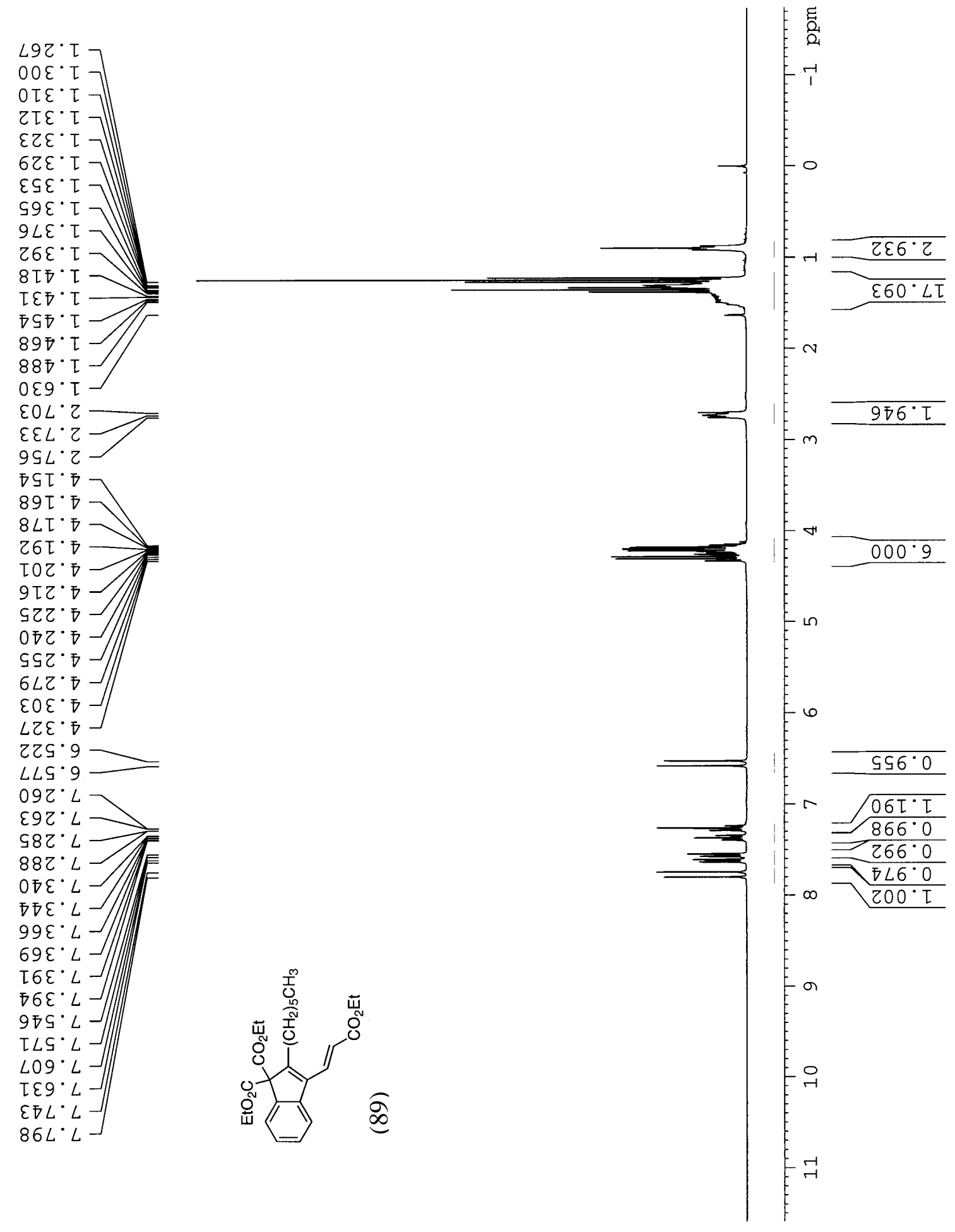




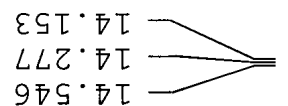

$\varepsilon \varepsilon 8^{\circ} \mathrm{zZ}$

$\nabla \varsigma \varepsilon \cdot 8 Z$

हटT.

62L. $0 \varepsilon$

万乙८ $\tau \varepsilon$

$\varepsilon 88^{\circ} 09$

$807 \cdot 29$

T00 $\mathrm{ZL}$

$\varepsilon \nabla 8 \cdot 9 L$

$892^{\circ} L L$
$Z 69^{\circ} L L$

OLL.OZT

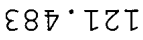

$\varepsilon \unlhd \varepsilon \cdot \varsigma 乙 \tau$

9乙币・9ZT

$898 \cdot 8 Z \tau$

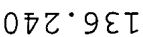

268.9عT

IST. ODT

6DL ZDT

SOZ.TST

$062 \cdot \angle 9 T$

$860^{\circ} 89 T$

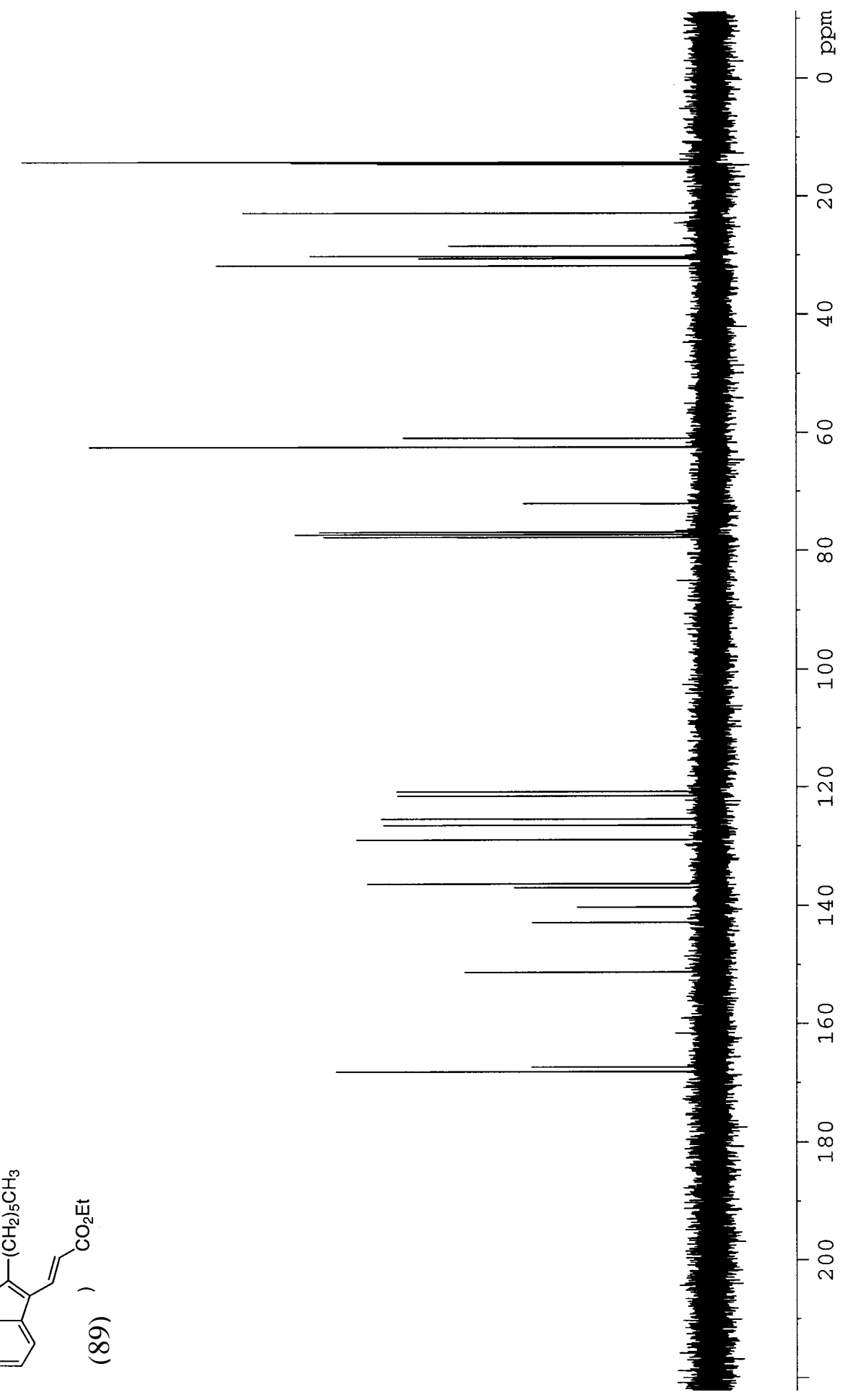

